THREE ESSAYS ON LABOR ECONOMICS

\author{
A Dissertation \\ presented to
}

the Faculty of the Graduate School

at the University of Missouri-Columbia

In Partial Fulfillment

of the Requirements for the Degree

Doctor of Philosophy

by

TAPAS PAUL

Dr. Peter Mueser, Dissertation Supervisor

JULY 2021 
The undersigned, appointed by the dean of the Graduate School, have examined the dissertation entitled

THREE ESSAYS ON LABOR ECONOMICS

presented by Tapas Paul,

a candidate for the degree of doctor of philosophy, and hereby certify that, in their opinion, it is worthy of acceptance.

Professor Peter Mueser

Professor Michael Podgursky

Professor Joan Hermsen

Professor Oded Gurantz 
..To my parents, Rama, and Dhruba. 


\section{ACKNOWLEDGEMENTS}

The journey of this dissertation writing deserves credit to many people for making this successful. First and foremost, I am indebted and owe a great deal of gratitude to my advisor, Peter Mueser. He is a well-known scholar and an exceptional mentor. In the fall of 2017, we agreed to work together for my dissertation on labor economics. Ever since, he is providing enthusiasm, motivation, and encouragement to my work. He showed me the scholarly scientific path for a dissertation. Peter spent over 160 weekly meetings thus far, and continually patiently reviewed my drafts, and always gave valuable comments. I always had a refreshing mood after finishing weekly meetings since I learned new things and sharpened my understanding. He and his wife Irene also took good care of my family. This is an excellent experience of my life to get doctoral training from him. I am fortunate enough to get an opportunity to work with him.

I want to thank Oded Gurantz for his time and guidance for my third chapter. Discussion with him was an excellent experience. I was impressed by his Stata coding expertise, and this inspired me. I sincerely acknowledge his contribution and guidance on my dissertation.

I acknowledge my sincere respect to two of my other committee members, Michael Podgursky and Joan Hermsen. They made a valuable contribution to my dissertation. I always enjoyed the discussion with Mike. Joan was always very supportive, and I took a class in Spring 2017 with her on poverty and inequality, which helped my understanding of the poverty and inequalities in the United States. 
I am thankful to Colleen Heflin for a smooth and successful transition from economics to public policy. It was an excellent and productive experience to work with her, despite only one semester long. Working with administrative data on SNAP was valuable.

I also thank my course instructors at the University of Missouri. My colleagues and classmates were also helpful, and they gave me a learning environment. I also learned from my students in the class. Truman School and the Institute of Public Policy were very supportive of me in pursuit of my degree.

Finally, I would like to thank my family, who always encourages me and extended their unconditional support and sacrifice for my work. To my mother: you made a great sacrifice at this age; I could not take good care of you since my departure from Bangladesh. To my wife: you always encourage me in my higher education studies in the United States and happily embraced our long-distance relationship for many years. Finally, at the half-way point to my degree, we reunited and started our family life. We are fortunate to have a new member in our family in 2020. 
TABLE OF CONTENTS

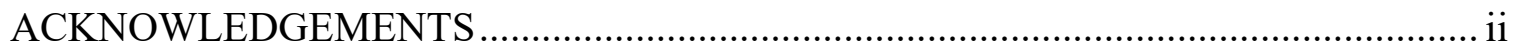

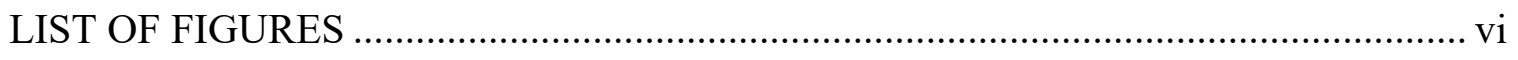

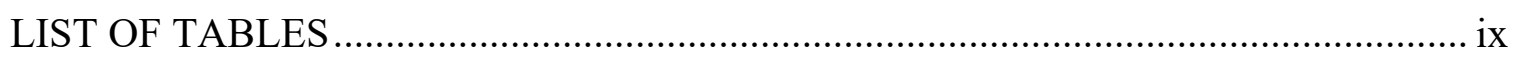

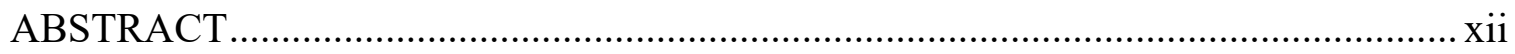

Chapter

1. THE ADDED WORKER EFFECT DURING THE GREAT RECESSION........... 1 Introduction

Literature Review

Data and Descriptive Statistics

Empirical Strategy

Results

The Added Worker Effect

Comparison of Study Estimates

Conclusion

References

Appendix A

Appendix B

2. THE IMPACT OF MARRIED WOMEN'S LABOR MARKET

PARTICIPATION ON POVERTY DURING THE GREAT RECESSION........ 55

Introduction

Literature Review

Poverty Measures in the United States

Data Collection

The Sample and Descriptive Statistics Data Collection

Methods

Results

Conclusion and Policy Implications 
References

Appendix A

Appendix B

Appendix C

Appendix D

3. DID EXPANDING THE SUPPLY OF PUBLISHING OPPORTUNITIES

INCREASE REPRESENTATION WITHIN THE ECONOMICS DISCIPLINE?

Introduction

Literature Review

Background on the American Economic Journal

Data

Methodology

Results

Main Treatment Effects

Robustness of Main Treatment Effects

Conclusion

References

Appendix

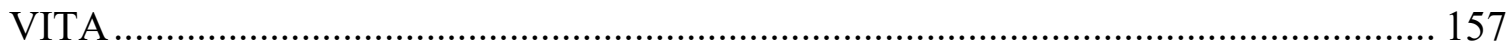




\section{LIST OF FIGURES}

Figure $\quad$ Page

Chapter 1

1. Odds ratios predicting wives' likelihood of labor force participation at $t$ as function of husband's labor force status (unemployment) in prior month, $(t-1)$ :

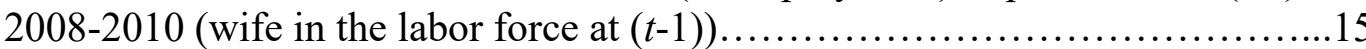

2. Odds ratios predicting wives' likelihood of labor force participation at $t$ as function of husband's labor force status (unemployment) in prior month, $(t-1)$ : 2008-2010 (wife not in the labor force at $(t-1)$ )..............................

3. Labor Force Participation Rates of Females and Males (age 25-54), 2000-2019

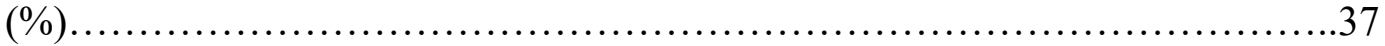

4. Labor Force Participation Rates of Females by Age, 2000-2019 (\%).............38

5. Labor Force Participation Rates for Females and Males by Race (age 25-54),

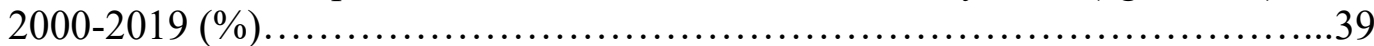

6. Unemployment Rates for Females and Males, 2000-2019...................40

7. Unemployment Rates of Female and Male by Race (25-54), 2000-2019 (\%)....41

\section{Chapter 2}

1. Distribution of poverty by month: percent of family in poverty based on official

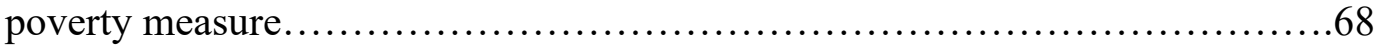

2. Proportion of households in month $t$ with husband labor force status.............70

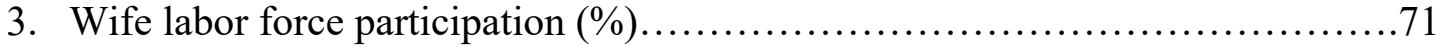

4. Effect of wife's earnings on the level of poverty based on official poverty measure... .78

5. Distribution of poverty by month: percent of family in poverty based on supplemental poverty measure.

6. Employed wives' mean earned income

7. Mean unemployment insurance benefits .95 


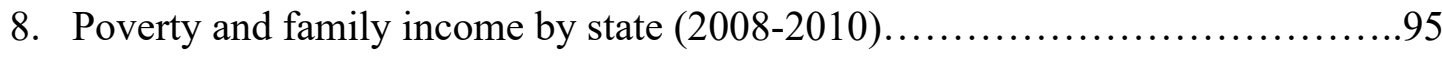

9. Effect of wife's earnings on the level of poverty based on supplemental poverty

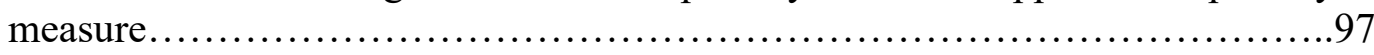

10. Distribution of poverty by month: percent of family in poverty based on supplemental poverty measure (omitting SNAP) ........................... 102

11. Effect of wife's earnings on the level of poverty based on supplemental poverty measure (omitting SNAP) ............................................... 103

12. Effect of wife's earnings on the level of poverty based on supplemental poverty measure (excluding income tax) ......................................... 104

Chapter 3

1. The average institutional adjusted rank of authors per journal article...........122

2. Average citations per journal publication................................. 123

3. Difference-in-difference event study impacts of AEJs on authors' from low

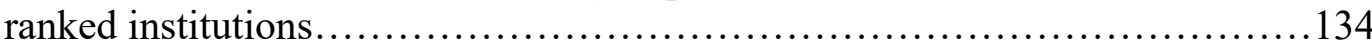

4. Fixed effect difference-in-difference event study impacts of AEJs on authors' from low ranked institutions............................................ 134

5. Difference-in-difference event study impacts of AEJs on authors' from low ranked institutions considering minimum author rank....................... 138

6. Fixed effect difference-in-difference event study impacts of AEJs on authors from low ranked institutions considering minimum author rank

7. Difference-in-difference event study impacts of AEJs on authors' from low ranked institutions considering maximum author rank

8. Fixed effect difference-in-difference event study impacts of AEJs on authors' from low ranked institutions considering maximum author rank.

9. Difference-in-difference event study impacts of AEJs on authors from low ranked institutions considering percent of articles published.........................145

10. Fixed effect difference-in-difference event study impacts of AEJs on authors from low ranked institutions considering percent of articles published.

11. Journal articles after reshaped into one row per author-article (\% annually).....151 
12. Highest similarity score $(>0.5)$ per institution..............................

13. Ability of Stata's 'matchit' procedure to link author's stated affiliation to institutional rank....................................................... 152

14. Average institutional rank of authors per journal article.................... 152 


\section{LIST OF TABLES}

Table

Page

Chapter 1

1. Summary Statistics......................................................

2. Odds ratios predicting wives' likelihood of labor force participation at $t$ as function of husband's labor force status in prior month(s), 2008, $2009 \& 2010 \ldots 18$

3. Odds ratios predicting wives' likelihood of labor force participation at $t$ as function of husband's labor force status in prior month(s), 2008 to 2010 . . .

4. Odds ratios from fixed effects logit predicting wives' likelihood of labor force participation at $t$ as function of husband's labor force status in prior month(s), 2008 to 2010

5. Odds ratios for fixed effects logit predicting wives' likelihood of labor force participation at $t$ as function of husband's labor force status in prior month(s), $2008,2009 \& 2010$

6. Odds ratios for logit predicting wives' likelihood of labor force participation at $t$ as function of husband's labor force status in prior month, 2008

7. Odds ratios for logit predicting wives' likelihood of labor force participation at $t$ as function of husband's labor force status in prior month, 2009 (wife in the labor force at $(\mathrm{t}-1))$.

8. Odds ratios for logit predicting wives' likelihood of labor force participation at $t$ as function of husband's labor force status in prior month, 2009 (wife not in the labor force at (t-1))

9. Odds ratios for logit predicting wives' likelihood of labor force participation at $t$ as function of husband's labor force status in prior month, 2010 (wife in the labor

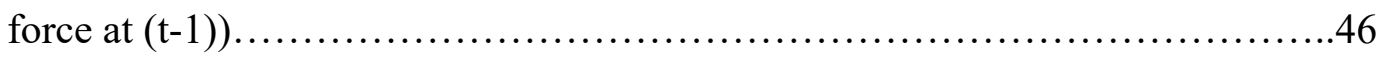

10. Odds ratios for logit predicting wives' likelihood of labor force participation at $t$ as function of husband's labor force status in prior month, 2010 (wife not in the labor force at (t-1))

11. Odds ratios predicting wives' likelihood of labor force participation at $t$ as function of husband's labor force status in prior month(s), 2008 
12. Odds ratios for logit predicting wives' likelihood of labor force participation at $t$ as function of husband's labor force status in prior month(s), 2009

13. Odds ratios predicting wives' likelihood of labor force participation at $t$ as function of husband's labor force status in prior month(s), 2010 .50

14. Odds ratios from logit predicting wives' likelihood of labor force participation at $t$ as function of husband's unemployment in prior month, (t-1): 2008, 2009 \&

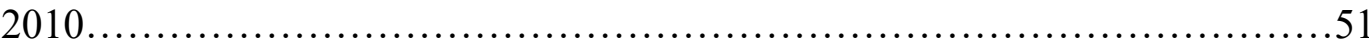

15. Average partial effect predicting wives' likelihood of labor force participation at $t$ as function of husband's labor force status in prior month(s), 2008 to $2010 \ldots \ldots .53$

16. Means for variables: wife in LF in $t$, husband employed in (t-1), husband unemployed in (t-1) \& husband not in the labor force in $(\mathrm{t}-1)$.

Chapter 2

1. Summary statistics. .64

2. Poverty decomposition $\left(t^{\prime}<t\right)$ for each month compare to first month $\left(t^{\prime}\right.$ : May 2008), Official Poverty Measure (OPM) .80

3. Poverty decomposition $\left(t^{\prime}<t\right)$ for each month compare to first month ( $t^{\prime}$ : May 2008), Supplemental Poverty Measure (SPM).

4. Poverty decomposition $\left(t^{\prime}<t\right)$ for each month compare to first month ( $t^{\prime}$ : May 2008), Supplemental Poverty Measure (SPM), with family income excluding SNAP

5. Poverty decomposition $\left(t^{\prime}<t\right)$ for each month compare to first month $\left(t^{\prime}\right.$ : May 2008), Supplemental Poverty Measure (SPM), with family income not excluding income tax

\section{Chapter 3}

1. Descriptive Statistics

2. Impacts of AEJs on articles average institutional ranking of the authors. 
3. Impacts of AEJs on articles average institutional ranking of the authors (minimum

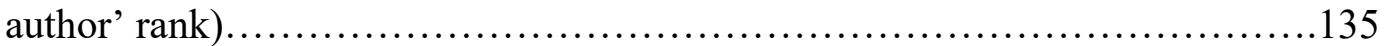

4. Impacts of AEJs on articles average institutional ranking of the authors (maximum author' rank) ................................................. 140

5. Impacts of AEJs on articles average institutional ranking of the authors (percent

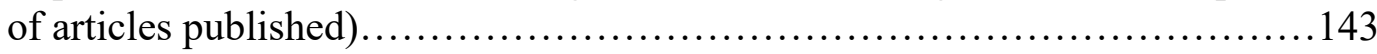

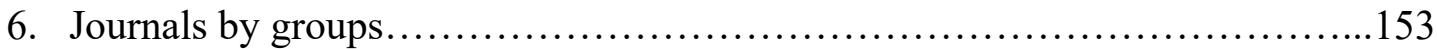




\title{
THREE ESSAYS ON LABOR ECONOMICS
}

\author{
Tapas Paul \\ Dr. Peter Mueser, Dissertation Supervisor
}

\begin{abstract}
This dissertation addresses labor market issues. The first two chapters deal with employment issues during the great recession using nationally representative data from the Survey of Income and Program Participation. The first chapter looks at the added worker effect in the great recession, the wife's labor market response to a husband loss of job. The second chapter investigates the impact of a wife's labor market participation on family poverty. The third chapter examines employment opportunities in the economics discipline using journal publication records from IDEAS/RePEc. It looks at the effect of new journal entry on the distribution of publications for universities of differing academic rank.
\end{abstract}




\section{CHAPTER 1: THE ADDED WORKER EFFECT DURING THE GREAT RECESSION}

\section{Introduction}

An individual's decision to participate in the labor market depends on her social and economic conditions, including her family characteristics, and the family's social and physical environment. Traditionally, wives are responsible for household activities and child-rearing, and husbands are primarily responsible for providing financial support. A married woman's choice of whether to work in the labor market is generally considered a joint decision of the family and, in many cases, to be a risk-sharing activity for the family. A married woman is often assumed to be more likely to work or to join the labor force if her husband becomes unemployed, depending on her prior labor force status (Cullen \& Gruber, 2000).

Woytinsky (1940) may be the first author referencing the "added worker effect" in the labor economics literature. For him, the idea of an added worker was identical with the term "forced entrant." Additional workers were the persons who entered the labor market because the usual breadwinner in the family was unemployed; otherwise, the person would not look for a job. In this study, the added workers are defined as wives who enter the labor force due to their husbands' job loss. The wife's labor market entry may be for a short or a long period. A household may accommodate to loss of income in several ways, and the wife's labor market entry is only one of them (Lundberg, 1985).

Historically, women's labor force participation was relatively low. In 1940 it began a strong and steady increase (Blau \& Winkler, 2017). Only $28 \%$ of women were in the

labor force in 1940 and this rose to $57.5 \%$ by 1990 . Many scholars report that in World War II women had to join the labor force to fill the vacant positions of men who left their 
civilian jobs (Blau \& Winkler, 2017). A shift in policy focusing on work requirements contributed to growing work motivation among welfare recipients in the 1970s and the 1980s. Women's labor force participation may have responded to two major legislative efforts in the 1990s (Cotter et al., 2004). First, the Family and Medical Leave Act of 1993 allowed women to move temporarily out of the labor force for qualified family reasons and medical leave with a guarantee their job would remain available (Department of Labor, n.d.). Second, the Personal Opportunity and Work Reconciliation Act of 1996 mandated a set of rules for the welfare system that required work in exchange of limited-time assistance (Department of Health \& Human Services, 2015). These policy initiatives were accompanied by other policy changes in the 1990s with an intention of enhancing employment opportunities and improving earning levels of welfare recipients and other disadvantaged people in the United States (Moffitt, 2002). In the appendix A, I discuss variation over time in female labor force participation and unemployment by age and race.

Among welfare programs, a few of them have direct or indirect labor supply effects. The Supplemental Nutrition Assistance Program (SNAP) is a food support program to maintain nutritious food for families. Studies of the effect of food stamp on labor supply have found mixed results; labor supply increased or decreased (Currie, 2003; Hoynes \& Schanzenbach, 2012). To be eligible for SNAP benefits individuals must meet some work requirements that include registration for work, prohibition on job quits, requirements to take a job if offered, and participation in a job training program if offered (USDA-FNS, n.d.). However, the eligibility conditions vary depending on personal characteristics and the economic environment. For example, during the Great Recession period, the benefits of SNAP were raised and the eligibility conditions were relaxed (Moffitt, 2015). 
Most studies have found an added worker effect, although the magnitude of the effect varies greatly. Studies defined the added worker effect as occurring when wives entered into the labor force due to their husbands' job loss (Cullen \& Gruber, 2000; Fernandes \& Felício, 2005). In the present paper, I examine the added worker effect during the Great Recession. While previous studies have investigated the labor supply of women responding to husbands' job loss, few of them have looked at recessionary periods; only Mattingly \& Smith (2010) looked at the Great Recession. Mattingly \& Smith (2010) looked only at women's employment changes over the two periods 2004-2005 and 2008-2009, and their study is hampered by the failure of the CPS to follow households that change address.

Using the Survey of Income and Program Participation (SIPP) data, the current analysis will give us the ability to look at monthly transitions of wives' labor market participation using a representative sample that follows movers. Our analysis allows us to provide a valuable update and extension of our knowledge of the added worker effect during in the largest economic downturn of the early twenty-first century.

\section{Literature Review}

A large number of studies have analyzed the effects of different factors on women's labor supply choice. Women's choice to participate in the labor market depends on various factors, including family characteristics, her own background, economic environment, and social policies. Cullen and Gruber (2000) listed at least three reasons for wives to enter the labor market in a situation where husbands become unemployed. First, husbands and wives may substitute their leisure through home production. Second, families face liquidity constraints when husbands are jobless and need to maintain fixed consumption 
commitments (Lundberg, 1985 and Mincer, 1962). Third, couples are responsive to unemployment and reemployment in the presence of perfect capital market. Their decisions are contingent to the lifetime prospects of these events in the life cycle model (Dynarski \& Sheffrin, 1987).

Estimates of the added worker effect vary greatly in prior studies. Generally, the estimates of added worker effect are higher for developing economies relative to developed economies. In the results section of this paper I discuss the variations of added worker effects and compare the estimates to this study findings. Two papers that used the recession-prosperity comparison made similar findings that the added worker effect is larger during economic downturns relative to economic prosperity (Mattingly \& Smith, 2010; Parker \& Skoufias, 2004). The added worker effect survives during the economic slowdown: during a slowdown women were more responsive during a job loss of the male partner but less responsive to a reduction of his earnings (Ghignoni \& Verashchagina, 2016). Two papers also found added worker effect during a high unemployment period (Lundberg, 1985; Spletzer, 1997).

Families can respond to the financial hardship in various ways and one of them is to increased labor supply by spouses. However, the magnitude of the added worker effect is related to the benefit and cost of alternative responses, such as husband undertaking an intensive job search or borrowing money from other sources (Lundberg, 1985). In a static model of household labor supply, the added worker effect occurs for two reasons: (a) an income effect where the income of the household declines due to husband's job loss and (b) a cross-substitution effect where within household activities the husband's time 
replaces the wife's time (Fernandes \& Felício, 2005; Karaoglan \& Okten, 2015; Lundberg, 1985).

The presence of young children is a strong negative predictor of wives' labor supply. Wives' labor market response following husbands unemployment is much larger for families with young children, as the wife is much less likely to be working. The standard models of allocation of time also shows that wives' labor force participation is reduced by the presence of young children (Cullen \& Gruber, 2000; Lundberg, 1985; Mankart \& Oikonomou, 2016; Mattingly \& Smith, 2010).

Women endowed with higher human capital due to higher schooling levels will achieve higher earnings, and will therefore be more likely to work. In fact, labor supply increases with the education level of the wife (Cullen \& Gruber, 2000; Mankart \& Oikonomou, 2016; Mattingly \& Smith, 2010; Smith \& Mattingly, 2014; Spletzer, 1997). Greater wife's education leads to a higher likelihood of transition from inactivity to activity (Fernandes \& Felício, 2005). Smith and Mattingly (2014) found that in three different recessionary periods show the same direction of differences of wives' likelihood of entering the labor force due to greater education. In many cases, the husband's education is also a good predictor for wife's choice of labor market participation. Wives' of more educated husbands' have higher labor market participation due to their marriage preference (Cullen \& Gruber, 2000).

The coefficients for age and age squared indicate a standard inverted U predicting the chance of wife's transition from inactivity to activity with wife's age (Cullen \& Gruber, 2000). Increasing age has a negative effect on wives' likelihood of joining the labor force in response to husbands' transition from employment to unemployment (Spletzer, 1997). 
Parker and Skoufias (2004) findings didn't support the standard inverted U for the probability of wives' transition from inactivity to activity (age coefficient is negative and age-squared is positive). Mankart and Oikonomou (2016) found that wives' age has a negative effect on the probability of joining the labor force.

Mankart and Oikonomou (2016) found that the likelihood of joining the labor force is higher for black wives than for white wives while measuring the added worker effect. Cullen and Gruber (2000) also found that white wives' likelihood of labor market participation is lower relative to that of black wives. Compared to white wives, non-white wives have $5.3 \%$ higher chance of joining the labor force when a husband loses his job (Spletzer, 1997). Wives in black families were more likely to seek a job during the recession than white wives and it was difficult for black wives to get jobs during the Great Recession (Mattingly \& Smith, 2010).

While previous studies investigated the labor supply of women responding to husbands' job loss, few of them explicitly looked at the recessionary periods. Within the papers that investigated recessions, they did not pay attention to monthly transitions (from not in labor force to labor force) of wives. The Survey of Income and Program Participation data will give us the ability to look at the monthly transitions of wives' employment. To our knowledge, however, both the theoretical and empirical studies have ignored the potentially important role of an added worker effect during the Great Recession. The added worker effect during the Great Recession remains largely unstudied. By examining monthly data, it can be helpful in addressing the transitions of couples' during the recessionary period and may help us to think about policy impacts in a period of economic slowdown (Spletzer, 1997). 


\section{Data and Descriptive Statistics}

The source of data used in this study is the 2008 Survey of Income and Program Participation conducted by the U.S. Census Bureau. ${ }^{1}$ SIPP is a household-based survey designed as a continuous series of national panels which provides interviews of the civilian noninstitutionalized population living in the United States since its inception in 1983 (US Census Bureau, n.d.). The SIPP is based on a nationally representative sample followed for approximately four years. It provides data on basic demographic and social characteristics for each member of the household during the survey period. These include age, sex, race, ethnic origin, marital status, relationship within the household, education, and veteran status. SIPP collects information for assistance received either directly as money or indirectly as in-kind benefits.

The structure of the data file is "person-month" and makes available all the data on a monthly basis. Households and their characteristics are defined on a monthly basis. The current analysis considers wave 1 to wave 7 of the 2008 SIPP dataset. This paper addresses the time period of the Great Recession, which lasts eighteen months, from December 2007 to May 2009 (NBER, 2010). However, the negative effects of the Great Recession also continue after its official end date. Keeping this fact, this study considers the time period for SIPP from May 2008 to August 2010.

SIPP provides information on labor force status and allows us to track individuals' monthly employment status. Although the SIPP is subject to differential attrition, using sample weights for the estimates reduces the bias that may arise from sample attrition

\footnotetext{
${ }^{1}$ This study downloaded 2008 SIPP data from the National Bureau of Economic Research (NBER) website where NBER provides STATA data definition files for the convenience of researchers. The NBER website is http://www.nber.org/data/survey-of-income-and-program-participation-sipp-data.html.
} 
(Czajka et al., 2008). Additionally, SIPP oversampled low-income people, and using sample weights adjusts for this issue (SIPP 2008, n.d.). Sample weights must be used to produce a nationally representative sample (Cameron \& Trivedi, 2010). In the current paper, I have used the household weights and all the analysis are based on weights. The household weights are important as my analysis is looking at the household level and particularly husband and wife, and any children.

The benefits of using the SIPP data are many. First, it has a shorter recall period than the Current Population Survey (CPS). Households need to remember information in only the prior four months, whereas in the CPS it requires to remember 12 months information. Second, SIPP tracks movers and the CPS does not track movers (Mattingly \& Smith, 2010). Third, SIPP has a sample that is representative of the U.S. population, but with households from high poverty areas oversampled, improving the power of analyses, like ours, that focus on the effects of unemployment. Fourth, the SIPP survey is particularly focused on obtaining information regarding receipt of government welfare programs.

I have applied some restrictions on the data for my analysis. In the first step, I consider a sample in which husbands and wives were both between 25 and 54 years of age. This allows me to remove most students and people who are retired (Cullen \& Gruber, 2000). ${ }^{2}$ In the next step, I limit my sample to married couples who are living together. They are known as "family household married couple."” To define employment, unemployment

\footnotetext{
${ }^{2}$ There is no consensus about the age of couples for the added worker effect as studies use various age groups for their analyses. For couples, Fernandes and Felício (2005) use ages 25 to 60, Gong (2011) uses ages 22 to 59, Mattingly and Smith (2010) use ages 18 to 65, Parker and Skoufias (2004) use ages 20 to 65, Karaoglan and Okten (2015) use ages 20 to 54, Mankart and Oikonomou (2016) use ages 25 to 55.

${ }^{3}$ Possible household types in the SIPP dataset are family hh-married couple, family hh-male householder, family hh-female householder, nonfamily hh-male householder, nonfamily hh-female householder (SIPP 2008 , n.d.).
} 
and labor force status, I use the definition used by the Survey of Income and Program Participation.

In the case of wives, I define two broader classes of wives, those in the labor force and those not in the labor force. Wives who are in the labor force consist of both employed and unemployed wives. There is a small sample of unemployed wives, about $5 \%$ of the observation. The transition from not in the labor force to the labor force is central to my analysis. In addition to that, during the Great Recession and afterwards, it was hard to obtain a job when the economy was shrinking. In contrast, I retain three categories of employed, unemployed, and not in the labor force for husbands. This will allow me to look at wives' choices conditional on whether their husbands are employed or unemployed.

Table 1. Summary Statistics

\begin{tabular}{lcc}
\hline & $\begin{array}{c}\text { Husband employed } \\
(1)\end{array}$ & $\begin{array}{c}\text { Husband not employed } \\
(2)\end{array}$ \\
\hline Wife in labor force & 0.742 & 0.746 \\
Husband's age & 41.9 & 41.3 \\
Husband's unemployment insurance & $(8.1)$ & $(7.7)$ \\
Yes & $\ldots$ & 0.269 \\
No & $\ldots$ & 0.730 \\
Wife's characteristics & & \\
Wife's education & & \\
Less than high school & 0.078 & 0.141 \\
High school graduate & 0.199 & 0.262 \\
Some college & 0.341 & 0.346 \\
Bachelor degree & 0.257 & 0.167 \\
Masters or higher & 0.126 & 0.082 \\
Wife's age & 39.5 & 40.0 \\
Wives' race and ethnicity & $(7.8)$ & $(8.1)$ \\
White alone & & \\
Black alone & 0.847 & 0.790 \\
Asian alone & 0.069 & 0.111 \\
Other races or more than one & 0.056 & 0.059 \\
Family variables & 0.027 & 0.039 \\
Number of children under 18 & & \\
& &
\end{tabular}




\begin{tabular}{|c|c|c|}
\hline 0 child & 0.308 & 0.372 \\
\hline 1 child & 0.245 & 0.237 \\
\hline 2 children & 0.282 & 0.232 \\
\hline 3 or more children & 0.163 & 0.158 \\
\hline \multicolumn{3}{|l|}{ Residence } \\
\hline Metro & 0.808 & 0.787 \\
\hline Not Metro & 0.156 & 0.174 \\
\hline Not Identified & 0.035 & 0.038 \\
\hline \multicolumn{3}{|l|}{ Ownership of the house } \\
\hline Owned or being bought & 0.787 & 0.633 \\
\hline Rented & 0.199 & 0.346 \\
\hline Occupied without payment & 0.013 & 0.020 \\
\hline No. of observations & 229,825 & 31,073 \\
\hline
\end{tabular}

Table 1 presents summary statistics for the sample that satisfies the sample restrictions described above for the first seven waves of the 2008 SIPP. All the subsequent analyses in this paper uses household weights. There are two groups of wives, wives whose husbands are employed, in first column, and wives whose husbands are not employed, in second column. Husbands not employed include unemployed husbands and husbands not in the labor force. On average, wives of husbands who are not employed work in $74.6 \%$ of the months in the SIPP data. Wives of husbands who are employed work $74.2 \%$ of the months. Several other characteristics are similar for wives with employed husbands and husbands not employed. These include husband's age, wife's age, wife's race and ethnicity, number of children under 18, residence and ownership of the house.

\section{Empirical Strategy}

Considering the availability of longitudinal data, I work with an identification strategy that will give me an opportunity to infer the causal effect of a husband's unemployment on his wife's labor force participation. The empirical analysis is based on 
logistic regression using the sample of wives who were out of the labor force for at least one month (Parker \& Skoufias, 2004). I use the following model:

$$
y_{i t}^{f}=f\left(\alpha+\beta_{1} u_{i(t-1)}^{h}+\beta_{2} N L F_{i(t-1)}^{h}+\delta_{i} \boldsymbol{X}_{i t}+\varepsilon_{i t}\right)
$$

where the dependent variable $y_{i t}^{f}$ is the wife's likelihood of being in the labor force at $t$ (month). The independent variable $u_{i(t-1)}^{h}$ is a binary variable set equal to 1 if the husband is unemployed at $(t-l)$ and 0 if he is not. Another binary variable $N L F_{i(t-1)}^{h}$ equals 1 if husband is not in the labor force at $(t-1)$ and 0 otherwise. Here, husband's labor force status is a categorical variable for the prior month $(t-1)$ identifying whether the husband was employment $(E)$, unemployment $(U)$, or not in the labor force $(N L F)$ in prior month. This is our key predictor in the analysis. I consider the category "husband is employed at prior month (t-1)" as a reference or base category. By definition, for an added worker effect we are interested in looking at wife's labor force choice in the current month $(t)$ as a result of her husband's unemployment in the prior month $(t-1)$.

Wife's choice of labor force participation also depends on other factors (covariates). $\boldsymbol{X}_{i t}$ is a vector of individual and household characteristics. In this analysis, in some models, I also include husband's unemployment for the prior two months $(t-l)$ and $(t-2)$. The vector $\boldsymbol{X}_{i t}$ includes husband's age and his highest level of schooling completed, wife's years of schooling, her age and age squared, her race and ethnicity. Family variables are also included in the vector $\boldsymbol{X}_{i t}$. This includes the number of children under 18 years of age, and whether the house is rented or owned. To represent local economic activity, I include the state unemployment rate. $\varepsilon_{i t}$ is a random component summarizing the influence of unobservables, and $\alpha, \beta$, and $\gamma$ are parameters to be estimated. The parameter $\beta_{1}$ is an 
estimate of the added worker effect, identifying the effect of the husband's unemployment on the labor force participation of his wife.

The advantage of this logit specification is that it allows us to establish a causal relation of exact timing of the wife's labor supply response to her husband's labor market status. Logit estimates of the odds ratio or marginal effects allow us to measure wives' likelihood of being in the labor force. In this paper, most of the results are presented in terms of odds ratios. I also present some results in terms of marginal effects for convenience in the appendix.

In the regression exercise, using equation 1 , I have five different models. In the first model, the wife is in the labor force in the prior month $(t-1)$. Husband's prior labor force status is represented by a categorical variable. The categorical variable has three different categories for the prior month $(t-1)$ : employed, unemployed and not in the labor force. I also include other covariates mentioned above. In the second model, wife is not in the labor force in the prior month $(t-1)$, with the covariates the same as first model. The second model is particularly important in this research as it captures the essence of the added worker effect. In the third model, wife's prior labor force status is not controlled. Here, I combine wives who are in the labor force and not in the labor force in prior month $(t-1)$, providing an overall measure of wife's decision without controlling wife's prior labor force status. The third model has more observations than the others, and so provides more precise estimates. In the fourth model, the structure is the same as in model one (wife is in the labor force in the prior month) except that I have an additional control identifying if husbands are unemployed in both prior months $(t-1)$ and $(t-2)$. This will give us a sense whether duration of unemployment matters or not to wife. The fifth model is based on model four 
with the only difference being that I consider wives who were not in the labor force in prior month $(t-1)$.

This paper analyzes the monthly survey data separately by year and with all years together. Using separate months will allow greater power in the combined regression exercise. We cluster errors by the individual.

In this study, I also considered interaction terms of wife's employment status and husband's labor force status. The regression equation with interactions is written:

$$
\begin{gathered}
y_{i t}^{f}=f\left(\alpha+\beta_{1} u_{i(t-1)}^{h}+\beta_{2} N L F_{i(t-1)}^{h}+\beta_{3} y_{i(t-1)}^{f}+\beta_{4}\left(y_{i(t-1)}^{f} * u_{i(t-1)}^{h}\right)+\beta_{5}\left(y_{i(t-1)}^{f}\right.\right. \\
\left.\left.* N L F_{i(t-1)}^{h}\right)+\delta_{i} \boldsymbol{X}_{i t}+\varepsilon_{i t}\right) \ldots \ldots \ldots \ldots(2)
\end{gathered}
$$

In the above logit model, the dependent variable is the probability of a wife being in the labor force in the current month $t$. Wife's employment status in the last period is defined by the $y_{i(t-1)}^{f}$. Within the same household, husbands' labor force status (unemployed and not in the labor force defined by $u_{i(t-1)}^{h}$ and $N L F_{i(t-1)}^{h}$, respectively, for the prior month $(t-1))$ is also considered. As defined in the earlier equations $\boldsymbol{X}$ is a vector of individual and household characteristics.

In the regression model of equation 2 , we have main effects and interaction effect. We are considering the estimates in terms of odds ratios. The intercept term $\alpha$ tells us that the odds of a wife being in the labor force in the current month for a husband who was employed in the prior month $(t-1)$ when the wife was not in the labor force in the prior month $(t-1)$. The intercept term captures excluded categories. The estimate of $\exp \left(\beta_{1}+\right.$ $\beta_{4}$ ) defines the odds of a wife's entry into the labor force in the current month when the wife was in the labor force in the last month $(t-1)$ and the husband was unemployed in the last month (t-1). The estimate of $\exp \left(\beta_{2}+\beta_{5}\right)$ shows the effect of the odds of a wife's 
entry into the labor force in the current month when the wife was not in the labor force in the last month and the husband was not in the labor force in last month.

This paper also include a logistic regression that includes household fixed effects. The benefit of using a fixed effect model is to control for time-invariant unobservable factors that may bias the analysis. In our context, the household fixed effects capture all unobservable, time-constant factors that affect wife's labor force status. For example, some wives may be highly motivated to get a job compared to other wives.

$$
y_{i t}^{f}=f\left(\alpha+\beta_{1} u_{i(t-1)}^{h}+\beta_{2} N L F_{i(t-1)}^{h}+a_{i}+\delta_{i} \boldsymbol{X}_{i t}+\varepsilon_{i t}\right) \ldots \ldots \ldots \ldots
$$

Here $a_{i}$ captures unobserved heterogeneity between households. The household fixed effects represents all factors affecting wife's labor force status that do not change over time. The vector $\boldsymbol{X}$ include husband's prior labor force status, wife's age, age squared, and state unemployment. Other variables, like race or ownership status of house, are dropped as they display no or very little variation over time.

\section{Results}

\section{The Added Worker Effect}

The added worker effect is addressed by looking at wife's labor supply response to her husband's unemployment. In the first step of my analysis, I construct monthly logistic analysis to address the added worker effect and wives' labor supply. The odds ratios are predicting wives' likelihood of labor force participation at time $t$ as a function of husbands' labor force status in the prior month $(t-1)$. Essentially an odds ratio indicates that the predictor changes the probability that the dependent variable is equal to 1 by a factor of on odds ratio. An estimate of 1 indicates no effect. The analysis looks at wives' prior labor force status for the last month, estimating the model on three samples: 1) wives who were 
in the labor force at $(t-1), 2)$ wives who were not in the labor force at $(t-1)$, and 3$)$ wives in either prior labor force status, but controlling for the direct effect of that status. For example, the analysis predicts wives' likelihood of labor force participation in August 2008 as a function of husbands' labor force status in July 2008. The estimated odds ratios are in figure 1 (wife in the labor force in prior month) and figure 2 (wife not in the labor force in prior month) with the $95 \%$ confidence interval. Details of the results are presented at appendix B, tables A1 to A5.

Figure 1. Odds ratios predicting wives' likelihood of labor force participation at $t$ as function of husband's labor force status (unemployment) in prior month, $(t-1)$ : 2008-2010 (wife in the labor force at $(t-1)$ ).

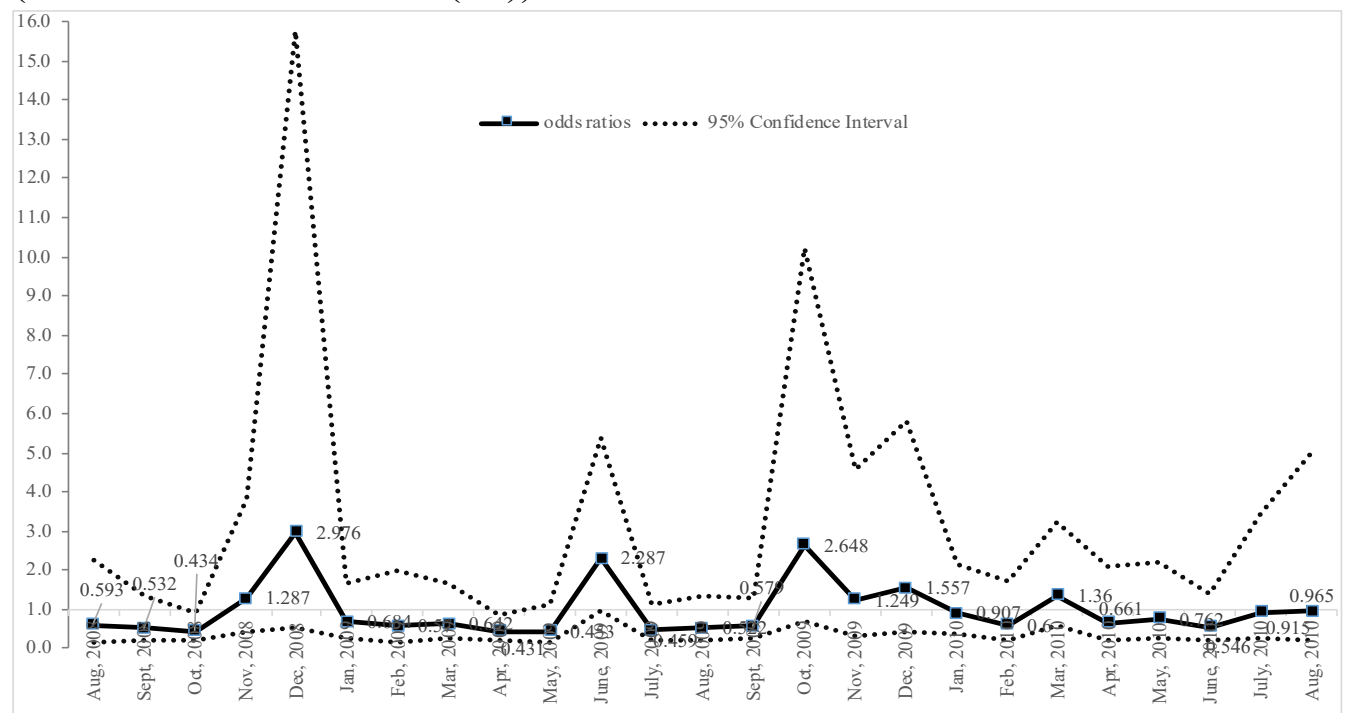

Notes: Black solid line presents odds ratios indicating the change in wives' odds of labor force participation in response to husbands' unemployment. The dotted lines encompass the $95 \%$ confidence interval. Monthly estimates based on equation (1). Detailed regression results are in tables A1 to A5 of appendix B.

In the monthly analysis I have results for twenty-five months based on surveys from August 2008 to August 2010. We have data starting from May 2008, but since we use data from prior months, the first month in which wife's labor force status is predicted is shifted forward. In those analyses using information only from one prior month, the first month 
for wife's labor force is June 2008; where three prior months are used, the first month is August 2008.

Figure 1 shows monthly odds ratios for wives likelihood of labor force participation in the current month $(t)$ as function of whether their husbands were unemployed, out of the labor force, or employment in the prior month $(t-1)$. Wives were in the labor force in the prior month $(t-1)$. The graph shows the how wives' entry into the labor force responds to their husbands' unemployment. If the ratio is over 1, wives' likelihood increases; if the ratio is under 1 , wives' likelihood decreases.

Figure 2. Odds ratios predicting wives' likelihood of labor force participation at $t$ as function of husband's labor force status (unemployment) in prior month, (t-1): 2008-2010 (wife not in the labor force at $(t-1)$ ).

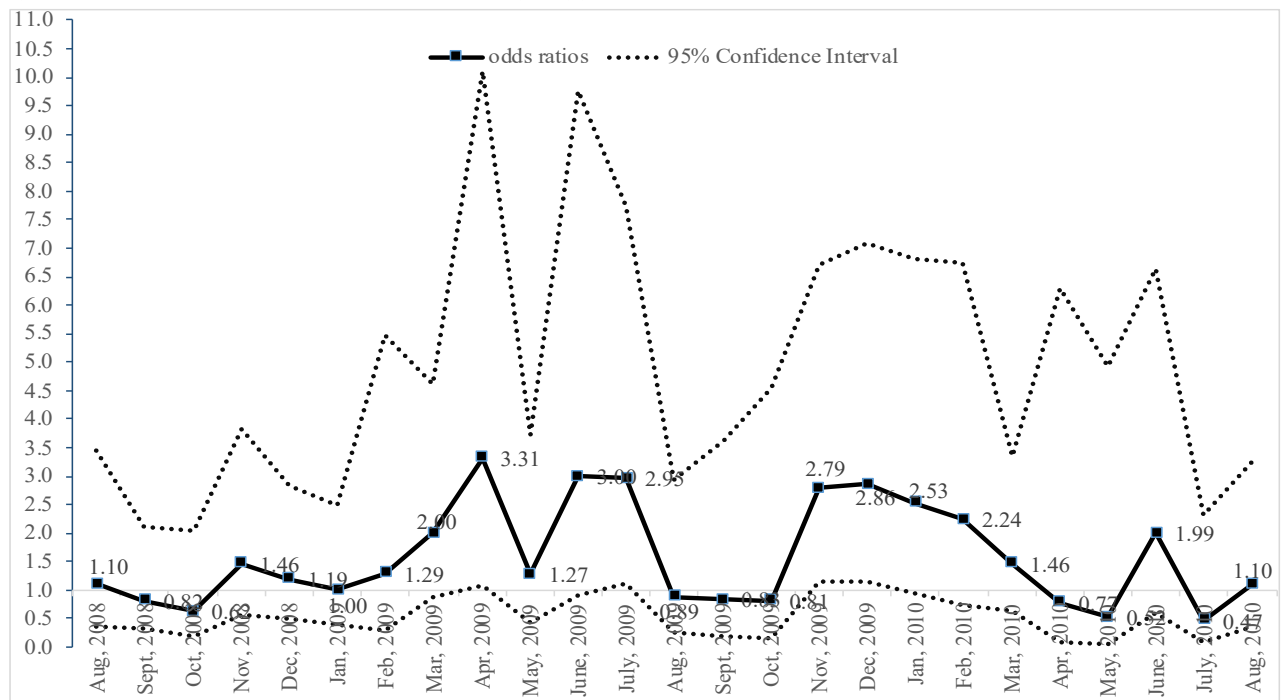

Notes: Black solid line presents odds ratios indicating wives' likelihood of labor force participation in response to husbands' unemployment. The grey dotted lines encompass the $95 \%$ confidence interval. Monthly estimates based on equation (1). Detailed regression results are in tables A1 to A5 of appendix B.

According to figure 2, for wives who were not in the labor force in the prior month $(t-1)$, their likelihood of being in the labor force increases in the current month $(t)$ if their husbands were unemployed. We find that the effect is statistically significant in four of the 
months. Husbands' job loss may be a key deciding factor for wives in deciding whether to join the labor force. However, we are not sure whether those wives obtained a job or not. They have a transition into the labor force from not in the labor force. Another possible scenario could be some wives may be fresh entrants, having joined the labor force for the first time, and some were in the labor force earlier. Those wives who were in the labor force before may have worked earlier and in response to husbands' smooth income flow they quit the job and moved out of the labor force.

Figure 1 and 2 allow comparison by the wife's labor force status in the prior month $(t-1)$. Figure 1 analyzes survey data where wives were in the labor force in the prior month $(t-1)$, whereas figure 2 considers the case where wives were not in the labor force in the prior month $(t-1)$. The size of estimates in figure 2 are generally greater than figure 1 . This could be interpreted as indicating that wives are responding more when they were not in the labor force in the prior month. Change in husbands' job status may lead wives to join the labor force or at least search for a job. For example, in figure 2 (table A3 of appendix B), the odds ratios for April 2009 is 3.3 and this is statistically significant at $p<0.05$. It mean that among wives not in the labor force in the prior month $(t-1)$, the odds of being in the labor force in current month when the husband is unemployed are 3.3 times as great as those when the husband is employed.

In these monthly results, the estimates are not very precise given that I have a small number of observations for each month. Number of observations will be increased if I work with annual data, combining months of the year. As a result, in the next step we move to the analysis based on annual data. 
The annual analysis is based on equation 1, and I present results in table 2 for three different years, 2008, 2009 and 2010. In this analysis, data for all months are combined for each year. In 2008, we have eight months of data from May 2008 to December 2008. In 2009, we have twelve months of data, and in 2010 we have eight months of data from January 2010 to August 2010. I present results for all three years separately. Detailed results are in the tables $\mathrm{A} 6, \mathrm{~A} 7$, and $\mathrm{A} 8$ of appendix $\mathrm{B}$. These analyses increase the precision of the estimates, reflected in smaller standard errors relative to the monthly analysis. In the combined analysis, we find a statistically significant added worker effect for 2009 but not 2008. In 2009, wives' chances of being in the labor force respond more strongly to their husbands unemployment in month $(t-1)$. Perhaps the fact that wives have observed the economy since the beginning of the Great Recession causes them to respond more aggressively in 2009. Also, we have more power, in terms of sample size, in 2009 relative to the years 2008 and 2010, so estimates for 2008 and 2010 are less precise.

Table 2. Odds ratios predicting wives' likelihood of labor force participation at $t$ as function of husband's labor force status in prior month(s), 2008, $2009 \& 2010$.

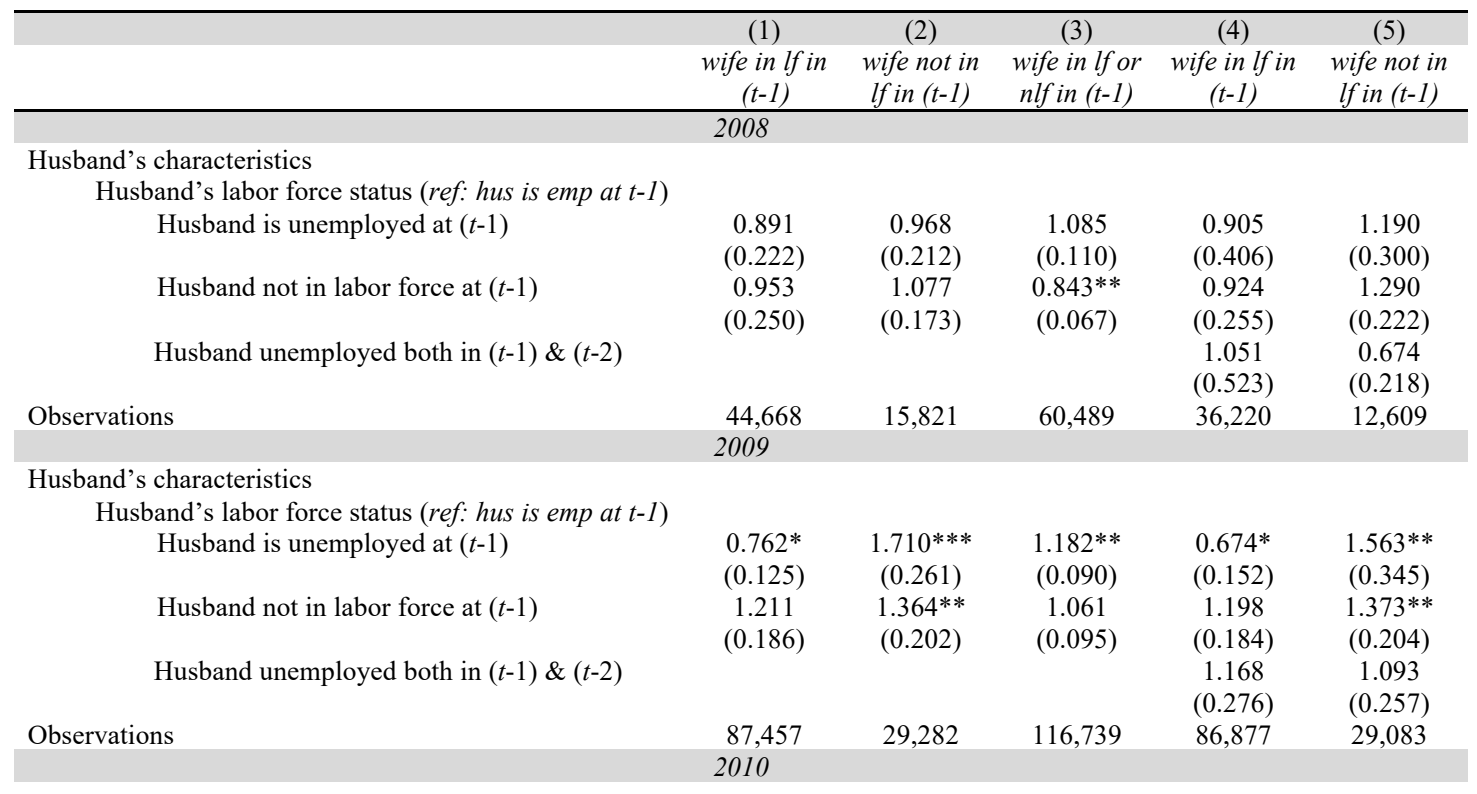


Husband's characteristics

Husband's labor force status (ref: hus is emp at t-1)

$\begin{array}{lccccc}\text { Husband is unemployed at }(t-1) & 0.827 & 1.332 & 1.277^{* * *} & 0.816 & 1.028 \\ \text { Husband not in labor force at }(t-1) & (0.148) & (0.243) & (0.114) & (0.251) & (0.310) \\ & 0.943 & 1.448^{* *} & 0.994 & 0.933 & 1.460 * * \\ & (0.171) & (0.243) & (0.093) & (0.170) & (0.247) \\ \text { Husband unemployed both in }(t-1) \&(t-2) & & & 1.042 & (0.355) & (0.424) \\ & & & & 51,966 & 18,574\end{array}$

Notes: Standard errors in parentheses. ${ }^{* * *} \mathrm{p}<0.01,{ }^{* *} \mathrm{p}<0.05,{ }^{*} \mathrm{p}<0.1$

In the next step, I moved to the combined sample. This combines all available monthly data from May 2008 to August 2010. Combined monthly data allows us to get more precise estimates using equation 1 above. The results from combined data are in table $3 .{ }^{4}$

Table 3. Odds ratios predicting wives' likelihood of labor force participation at $t$ as function of husband's labor force status in prior month(s), 2008 to 2010.

\begin{tabular}{|c|c|c|c|c|c|}
\hline & $\begin{array}{l}(1) \\
\text { wife in lf in } \\
(t-1)\end{array}$ & $\begin{array}{c}(2) \\
\text { wife not in } \\
\text { lf in }(t-1)\end{array}$ & $\begin{array}{c}\text { (3) } \\
\text { wife in lf or } \\
\text { nlf in }(t-1)\end{array}$ & $\begin{array}{c}\text { (4) } \\
\text { wife in lf } \\
\text { in }(t-1)\end{array}$ & $\begin{array}{c}(5) \\
\text { wife not in } \\
\text { lf in }(t-1)\end{array}$ \\
\hline \multicolumn{6}{|l|}{ Husband's characteristics } \\
\hline \multicolumn{6}{|l|}{ Husband's labor force status (ref: hus is emp at $t-1$ ) } \\
\hline \multirow[t]{2}{*}{ Husband is unemployed at $(t-1)$} & $0.797 * *$ & $1.421 * * *$ & $1.190 * * *$ & $0.743^{*}$ & $1.325^{*}$ \\
\hline & $(0.091)$ & $(0.155)$ & $(0.077)$ & $(0.128)$ & $(0.207)$ \\
\hline \multirow[t]{2}{*}{ Husband not in labor force at $(t-1)$} & 1.066 & $1.311 * * *$ & 0.982 & 1.050 & $1.385 * * *$ \\
\hline & $(0.124)$ & $(0.135)$ & $(0.069)$ & $(0.124)$ & $(0.146)$ \\
\hline \multirow[t]{2}{*}{ Husband unemployed both in $(t-1) \&(t-2)$} & & & & 1.106 & 1.068 \\
\hline & & & & $(0.222)$ & $(0.180)$ \\
\hline \multicolumn{6}{|l|}{ Husband's education (ref: some college) } \\
\hline \multirow[t]{2}{*}{ Less than high school } & $0.742 * * *$ & 0.977 & $0.733 * * *$ & $0.733 * * *$ & 0.975 \\
\hline & $(0.074)$ & $(0.094)$ & $(0.058)$ & $(0.074)$ & $(0.097)$ \\
\hline \multirow[t]{2}{*}{ High school graduate } & 0.951 & 1.100 & 1.083 & 0.958 & 1.102 \\
\hline & $(0.063)$ & $(0.089)$ & $(0.064)$ & $(0.064)$ & $(0.090)$ \\
\hline \multirow[t]{2}{*}{ Bachelor degree } & 1.076 & $0.689 * * *$ & $0.740 * * *$ & 1.084 & $0.695 * * *$ \\
\hline & $(0.080)$ & $(0.060)$ & $(0.046)$ & $(0.083)$ & $(0.061)$ \\
\hline \multirow[t]{2}{*}{ Masters or higher } & $0.759 * * *$ & $0.580 * * *$ & $0.432 * * *$ & $0.769 * *$ & $0.594 * * *$ \\
\hline & $(0.080)$ & $(0.061)$ & $(0.034)$ & $(0.081)$ & $(0.063)$ \\
\hline \multirow[t]{2}{*}{ Husband's age } & 1.002 & 1.004 & 1.001 & 1.003 & 1.005 \\
\hline & $(0.006)$ & $(0.006)$ & $(0.004)$ & $(0.007)$ & $(0.006)$ \\
\hline \multirow[t]{2}{*}{ Husband's unemployment insurance (ref: no ins.) } & 1.129 & 0.925 & 1.041 & 1.123 & 0.943 \\
\hline & $(0.162)$ & $(0.120)$ & $(0.079)$ & $(0.163)$ & $(0.124)$ \\
\hline \multicolumn{6}{|l|}{ Wife's characteristics } \\
\hline \multicolumn{6}{|l|}{ Wife's education (ref: some college) } \\
\hline \multirow[t]{2}{*}{ Less than high school } & $0.588 * * *$ & $0.681 * * *$ & $0.352 * * *$ & $0.587 * * *$ & $0.698 * * *$ \\
\hline & $(0.055)$ & $(0.079)$ & $(0.031)$ & $(0.056)$ & $(0.083)$ \\
\hline \multirow[t]{2}{*}{ High school graduate } & $0.826 * * *$ & $0.825 * * *$ & $0.584 * * *$ & $0.817 * * *$ & $0.835 * *$ \\
\hline & $(0.050)$ & $(0.058)$ & $(0.030)$ & $(0.050)$ & $(0.060)$ \\
\hline \multirow[t]{2}{*}{ Bachelor degree } & $1.255 * * *$ & $1.297 * * *$ & $1.343 * * *$ & $1.255 * * *$ & $1.271 * * *$ \\
\hline & $(0.096)$ & $(0.106)$ & $(0.083)$ & $(0.098)$ & $(0.107)$ \\
\hline \multirow[t]{2}{*}{ Masters or higher } & $1.964 * * *$ & $1.604 * * *$ & $2.623 * * *$ & $1.957 * * *$ & $1.569 * * *$ \\
\hline & $(0.234)$ & $(0.187)$ & $(0.260)$ & $(0.234)$ & $(0.193)$ \\
\hline \multirow[t]{2}{*}{ Wife's age } & $1.140 * * *$ & $1.073 *$ & $1.184 * * *$ & $1.138 * * *$ & $1.075^{*}$ \\
\hline & $(0.038)$ & $(0.040)$ & $(0.038)$ & $(0.039)$ & $(0.040)$ \\
\hline
\end{tabular}

\footnotetext{
${ }^{4}$ I also looked at the average partial effect from the logistic regression for convenience (presented in table A10 of appendix B). I find statistically significant added worker effects in this model of combined data. This coefficient of average partial effect tells us that wife has $1.5 \%$ higher chance of joining in the labor force if her husband is unemployed in the prior month.
} 


\begin{tabular}{|c|c|c|c|c|c|}
\hline Wife's age square ${ }^{(a)}$ & $\begin{array}{c}0.873 * * * \\
(0.036)\end{array}$ & $\begin{array}{c}0.885 * * \\
(0.043)\end{array}$ & $\begin{array}{c}0.815^{* * *} * \\
(0.033)\end{array}$ & $\begin{array}{c}0.875 * * * \\
(0.037)\end{array}$ & $\begin{array}{c}0.882 * * \\
(0.043)\end{array}$ \\
\hline \multicolumn{6}{|c|}{ Wife's race \& ethnicity (ref: White alone) } \\
\hline Black & $\begin{array}{c}0.942 \\
(0.100)\end{array}$ & $\begin{array}{c}1.463 * * * \\
(0.148)\end{array}$ & $\begin{array}{c}1.416 * * * \\
(0.121)\end{array}$ & $\begin{array}{c}0.946 \\
(0.102)\end{array}$ & $\begin{array}{c}1.463 * * * \\
(0.154)\end{array}$ \\
\hline Asian & $\begin{array}{c}0.796 * * \\
(0.088)\end{array}$ & $\begin{array}{c}0.747 * * * \\
(0.081)\end{array}$ & $\begin{array}{c}0.620 * * * \\
(0.057)\end{array}$ & $\begin{array}{c}0.799 * * \\
(0.089)\end{array}$ & $\begin{array}{c}0.778 * * \\
(0.084)\end{array}$ \\
\hline Other races or more than one & $\begin{array}{c}0.754 * * \\
(0.104)\end{array}$ & $\begin{array}{c}1.107 \\
(0.161)\end{array}$ & $\begin{array}{c}0.971 \\
(0.116)\end{array}$ & $\begin{array}{l}0.775 * \\
(0.107)\end{array}$ & $\begin{array}{c}1.104 \\
(0.162)\end{array}$ \\
\hline \multicolumn{6}{|c|}{ Family variables } \\
\hline 0 child & $\begin{array}{l}1.126 * \\
(0.076)\end{array}$ & $\begin{array}{c}1.581 * * * \\
(0.115)\end{array}$ & $\begin{array}{c}1.855^{* * *} * \\
(0.110)\end{array}$ & $\begin{array}{c}1.107 \\
(0.077)\end{array}$ & $\begin{array}{c}1.602 * * * \\
(0.119)\end{array}$ \\
\hline 1 child & $\begin{array}{c}0.955 \\
(0.062)\end{array}$ & $\begin{array}{c}1.217 * * * \\
(0.088)\end{array}$ & $\begin{array}{c}1.237 * * * \\
(0.063)\end{array}$ & $\begin{array}{c}0.939 \\
(0.061)\end{array}$ & $\begin{array}{l}1.209 * * \\
(0.090)\end{array}$ \\
\hline 3 or more children & $\begin{array}{c}0.796 * * * \\
(0.060)\end{array}$ & $\begin{array}{l}0.886 * \\
(0.060)\end{array}$ & $\begin{array}{c}0.586^{* * *} * \\
(0.033)\end{array}$ & $\begin{array}{c}0.783 * * * \\
(0.060)\end{array}$ & $\begin{array}{c}0.900 \\
(0.061)\end{array}$ \\
\hline \multicolumn{6}{|l|}{ Residence (ref: metro) } \\
\hline Not metro & $\begin{array}{c}0.968 \\
(0.065)\end{array}$ & $\begin{array}{c}1.009 \\
(0.081)\end{array}$ & $\begin{array}{c}1.107 \\
(0.078)\end{array}$ & $\begin{array}{c}0.987 \\
(0.068)\end{array}$ & $\begin{array}{c}1.009 \\
(0.080)\end{array}$ \\
\hline Not Identified & $\begin{array}{c}1.059 \\
(0.159)\end{array}$ & $\begin{array}{c}1.090 \\
(0.160)\end{array}$ & $\begin{array}{c}1.235 \\
(0.163)\end{array}$ & $\begin{array}{c}1.057 \\
(0.160)\end{array}$ & $\begin{array}{c}1.038 \\
(0.158)\end{array}$ \\
\hline Unemployment (state) & $\begin{array}{c}0.951 * * * \\
(0.010)\end{array}$ & $\begin{array}{c}0.962 * * * \\
(0.010)\end{array}$ & $\begin{array}{c}0.978 * * * \\
(0.007)\end{array}$ & $\begin{array}{c}0.965 * * * \\
(0.011)\end{array}$ & $\begin{array}{c}0.953 * * * \\
(0.011)\end{array}$ \\
\hline Constant & $\begin{array}{c}6.203 * * * \\
(3.785)\end{array}$ & $\begin{array}{c}0.020 * * * \\
(0.014)\end{array}$ & $\begin{array}{c}0.129 * * * \\
(0.079)\end{array}$ & $\begin{array}{c}5.421 * * * \\
(3.373)\end{array}$ & $\begin{array}{c}0.021 * * * \\
(0.015)\end{array}$ \\
\hline Observations & 184,424 & 63,793 & 248,217 & 175,063 & 60,266 \\
\hline
\end{tabular}

${ }^{(a)}$ Wife's age square is defined by age square divided by hundred.

The combined results in table 3 show that, during the recession, the odds that a wife enters the labor force are 1.4 times greater if her husband is unemployment in prior month $(t-1)$, which is statistically significant at $p<0.01$. On the other hand, wives who were in the labor force in the prior month are less likely to be in the labor force if their husbands were unemployed. If we don't control wives' prior labor force status then we find that the odds of wives being in the labor force are 1.2 times greater if their husbands are unemployment in the prior month. When we multiply the variable representing husband's unemployed in both prior months we see that the odds ratio is increased by a multiple of 1.42 (multiplication of 1.325 and 1.068), given that the wife was not in the labor force in prior month. This is the effect of having an unemployed husband in month $(t-1)$ and $(t-2)$ (and possibly more, of course) on wife's labor force participation. However, the increment associated with extended unemployment is not statistically significant. 
The controls for the logistic regression include husband's characteristics, wife's characteristics, family variables, residential status and state unemployment. Husbands' educational attainment has a significant effect on wives' labor force entry decisions. Husbands' higher educational attainments reduce the likelihood of wives' entry into the labor market. Families may have savings or a higher and more reliable income flow if husbands are highly educated. Husband's age does not affect wife's labor force choice as we see it is not statistically significant in any model. Wife's education plays an important role in her labor force entry choice. More educated wives are more likely to be in the labor force.

Wife's age has a significant effect to the labor force entry decision. The effect of wife's age on entry is captured by a quadratic equation. Wife's coefficient on age squared is negative, implying that the effect of age increases early on, reaching a peak, and then decline. The wife's choice of labor force participation increases with age up until about age 42 , and then decreases.

Race and ethnicity also influence wives' labor force entry choice. If the wife is black, then the odds ratio of her entering the labor market is about 1.46 times greater compared to a white wife, when wife was not in the labor force in prior month. However, if the wife is an Asian, then she has lower probability of entering the labor force. In the second section of this paper, I also discussed the fact that Asians have relatively better positions in terms of job security and are less likely to be affected by the Great Recession. It could be the case that Asian wives come from more affluent families.

Number of children plays an important role in wives' labor market entry decisions. In this analysis, I consider children who are under 18 years old. This age group is important 
as wives often take care of young children instead of engaging in labor force activities. The analysis shows that wives with more children are less likely to choose labor market entry.

Residence in a metropolitan area does not significantly influence the wife's labor force participation. Local economic activity as defined by the state's unemployment rate also plays an important role for wife's labor supply. The higher the unemployment, the lower the wife's likelihood of joining the labor force. During the Great Recession, job availability decreased particularly for low skilled workers, so getting a job was harder than in other periods.

In the next step, I consider the fixed effects in the logistic regression using equation 3. I combine all the observations from 2008 to 2010 . This study uses husband's labor force status, wife's age, number of children under age 18 and state unemployment as predictors in the fixed effects model. I dropped variables from this fixed effects model if they had little or no change over time. There may be selection bias due to households' unobserved characteristics (for instance, tastes for leisure, motivation, etc.) and the variables listed above may be unable to address the possible selection bias. This motivates me to use a fixed effects model. The results are presented in table 4. I grouped the data by household; the household fixed effects capture variation between households in labor force participation. In the table, coefficients are in terms of odds ratios.

Table 4. Odds ratios from fixed effects logit predicting wives' likelihood of labor force participation at $t$ as function of husband's labor force status in prior month(s), 2008 to 2010.

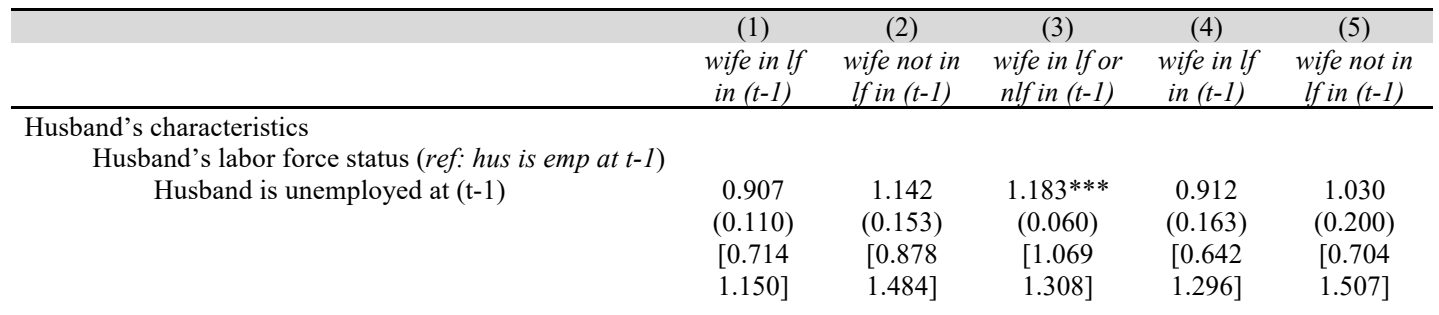




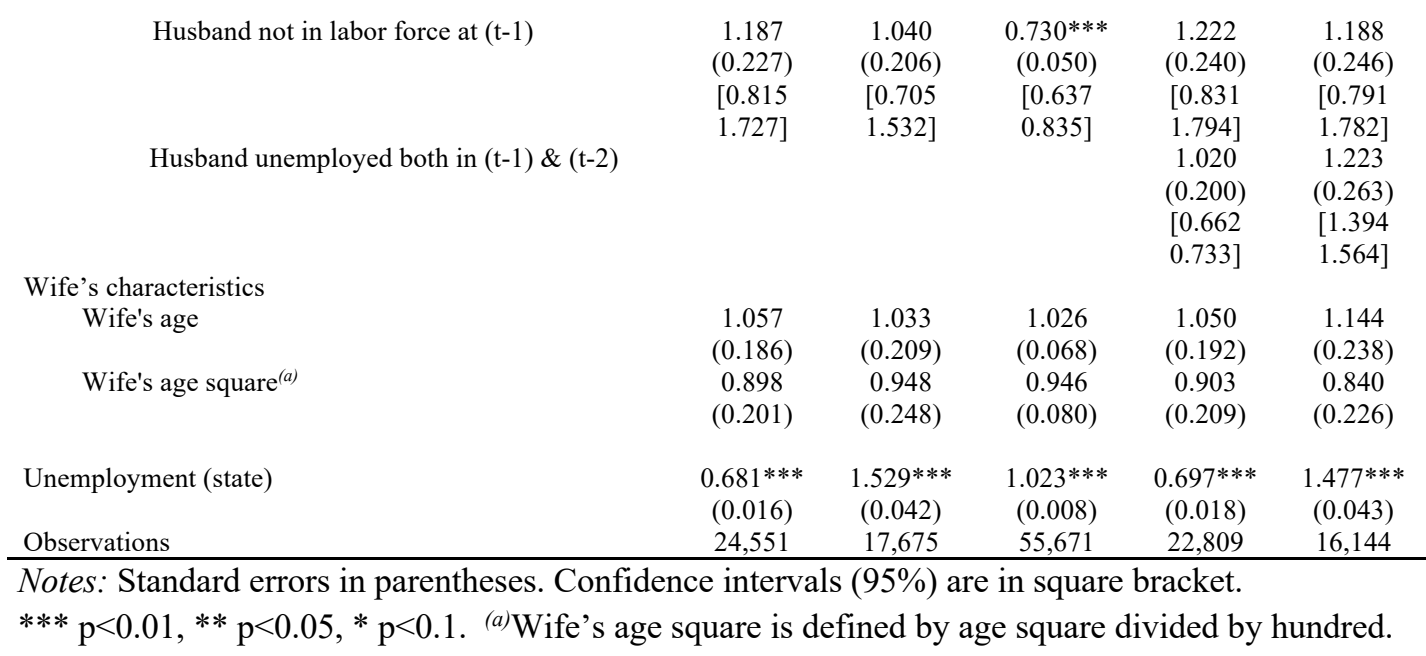

The fixed effect model shows that the odds of being in the labor force for wives with unemployed husbands are 1.14 times as great as those with an employed husband. However, the findings of the added worker effect from fixed effects logit models are not statistically significant. These results in table 4 are comparable to table 3 . In the logistic regression model presented in table 3 , we get statistical significant added worker effects, but when we use a fixed effects logit model, we do not find statistically significant the added worker effects.

Next, in the fixed effects model, I moved to annual analysis. Table 5 presents results from fixed effects for three different years of 2008, 2009 and 2010. These results are comparable to the annual results of table 2 . We see an added worker effect in the earlier model. However, fixed effects models do not confirm the presence of an added worker effect corresponding to the prior estimates. Only 2009 shows a statistical significant added worker effect. 
Table 5. Odds ratios for fixed effects logit predicting wives' likelihood of labor force participation at $t$ as function of husband's labor force status in prior month(s), 2008, 2009 $\& 2010$.

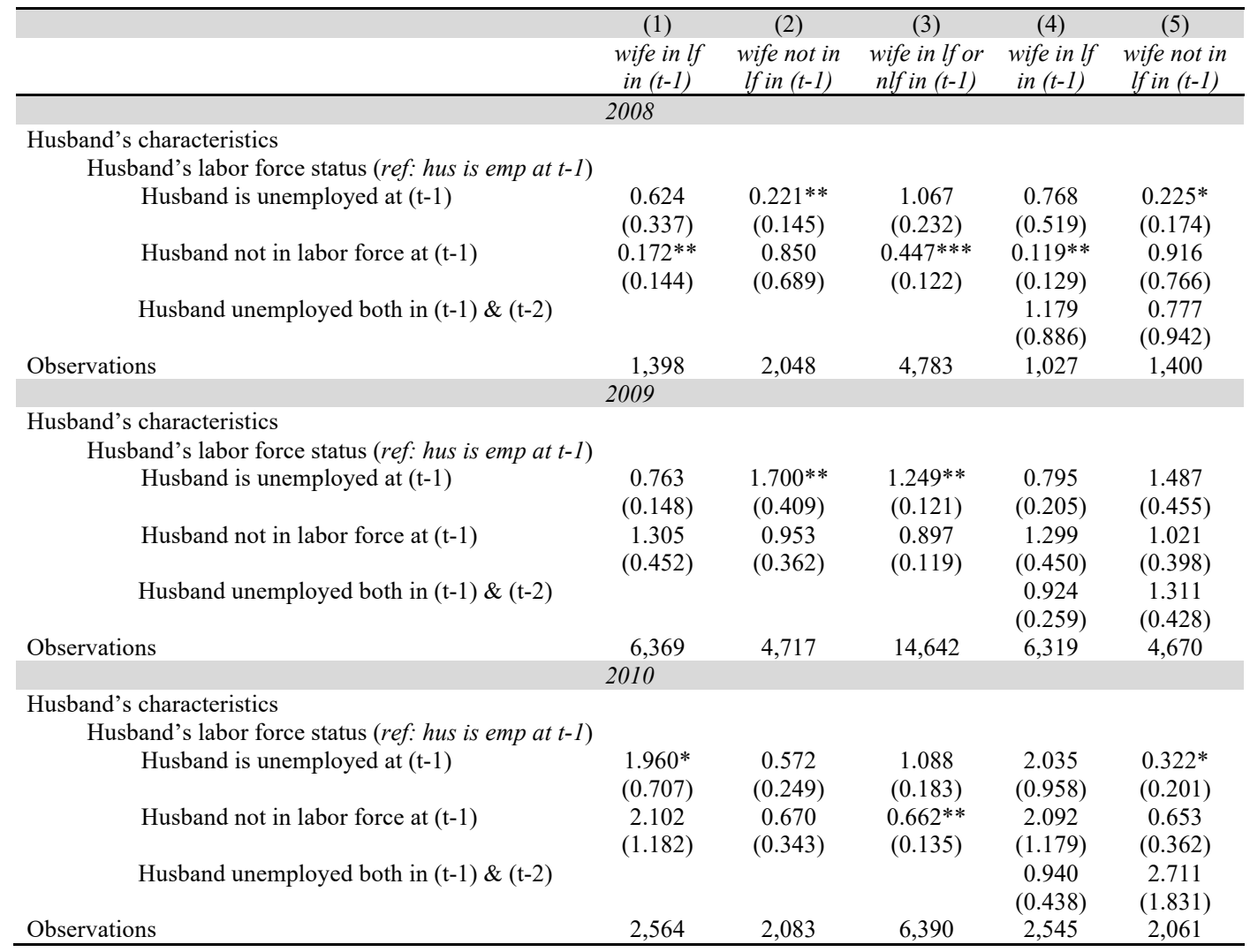

Notes: Standard errors in parentheses. ${ }^{* * *} \mathrm{p}<0.01,{ }^{* *} \mathrm{p}<0.05,{ }^{*} \mathrm{p}<0.1$

${ }^{(a)}$ Wife's age square is defined by age square divided by hundred.

Monthly dummies and their interactions with unemployed husbands are considered.

In the table A9 of appendix B, we have results from two different models, first model includes month and interaction terms, and second model includes month and interaction terms and with other controls. The table allows us to determine the degree to which coefficient estimates are robust to alternative models. We can interpret the coefficient of interaction of the husband's labor force status and the month dummies in columns (3) and (4). This tells us the odds ratios of a wife whose husband is employed of entering the labor force in August 2008 when the wife was in labor force in $(t-1)$ is greater by a multiple of 
1.487 than in December. ${ }^{5}$ The parameter associated with the interaction of August and husband unemployment is 0.759 , meaning that, for those wives who are not in the labor force in the prior month $(t-1)$, the odds of being in the labor force in the current month are 0.759 times as great for a wife with an unemployed husband as for a wife with an employed husband.

I also considered a model that looked at husbands' duration of unemployment. I looked at wife's likelihood of labor force participation as a function of the length of the husband's unemployment. I considered unemployment duration for zero to seven months or more. These additional measures did not contribute to the explanation of wife's labor force choice. The nature of the unemployment spells is that most are for short periods of time, so relatively few individuals were unemployed for six months or more. I couldn't find significant results for any duration of months. Once we consider durations of unemployment for six months or more, we see very few observations remaining for analysis. In the aggregated data set for seven waves of SIPP 2008, unemployed husbands constitute only $6.32 \%$ of the observations, and only $5.70 \%$ of husbands are not in the labor force.

\section{Comparison of Study Estimates}

In this section, I make an effort to compare the added worker effect among studies. This will give us a precise idea of the outcome measures, and how the added worker effect varies across studies. Studies may not be directly comparable due to different periods,

\footnotetext{
${ }^{5}$ logit $\boldsymbol{P}(Y=1)=\beta_{0}+\boldsymbol{\beta}_{1}$ month $+\beta_{2}$ husunemp $+\beta_{3}$ month $*$ husunemp $+X b$

This happens when an observation corresponds to an employed husband, for then unemployed $=0$.

$\operatorname{Pr}(w l f=1 \mid$ month $=$ aug2008, husband is unemployed $=0)-\operatorname{Pr}(w l f=1 \mid$ month $=\mathrm{dec} 2008$, husband is unemployed $=0)=(a+1.487+X b)-(a+0+X b)=1.487$

or, simply $\operatorname{Pr}(w l f=1 \mid$ month=aug2008, husband is unemployed=0) $=1.487$ (where, husband is employed)
} 
datasets, methods of analysis, and various economic environments. However, this section puts every effort into making them comparable.

Mankart and Oikonomou (2016) find an increase in the likelihood that a wife enters the labor force of between 4.7 and 8.3 percentage points, depending on the period, for the 1980 s to 2000 s, using a CPS data and a linear probability model. There is an ambiguity in the measurement of wife's labor force entry and the primary explanatory variable of husband's unemployment. Husband's is identified as entering unemployment based on his labor force status between months $t$ and $t+1$, and it appears that the wife's entry into the labor force is based on the same comparison. The added worker effect in their study that may corresponds to this current study is 7.8 percentage points for $2006-2009$, and this is higher than what I find in my study of 1.5 percentage point for the Great Recession, 20082010. There may be an error in their specification, as they did not allow wife's entry to vary by time period, and as a result, there is very likely an upward bias of the added worker effect.

Mattingly and Smith (2010) measure the principal independent variable as a husband who was employed at $t$, and became either unemployed or not in the labor force at $t+1$. Whereas, in the current study, I measure the husband's labor force status by two different measures, those being unemployed at $t-1$, or not in the labor force at $t-1$. Regarding the wife's labor force status, in their CPS dataset, $14.2 \%$ of wives who were not in the labor force entered into the labor force at time $t$. In this study, the SIPP data shows that only $5 \%$ of wives who was not in the labor force enter the labor force at time $t$. They find an odds ratio of 2 for the recessionary period, whereas the present study finds an odds ratio of 1.4 . 
A classic study by Lundberg (1985) finds a small but significant added worker effect for white families. The study shows that a wife has a 1.8 percentage points higher chance of entering the labor force if her husband is unemployed relative to an employed husband which is equivalent to a $25 \%$ higher chance of entering the labor force in a month. The current study finds a wife has a 1.5 percentage point higher chance of entering the labor force at time $t$ if her husband was unemployed at $t-1$, which is equivalent to about a $40 \%$ higher chance (using table A11 of appendix B). The difference may emanate from the differences in the dataset. The present study uses a nationally representative sample from the SIPP, and, in her study, Lundberg uses survey data from the Seattle and Denver Income Maintenance Experiments (SIME/DIME).

Spletzer (1997) estimates an added worker effect of 6 percentage points using CPS data for 1988 through 1991. Conversely, the current study estimates 1.5 percentage points for the Great Recession. Both of these studies use a similar definition of the added worker effect. However, we see a significant difference in the labor force characteristics of married wives. Spletzer's CPS data show, among the married women who are not in the labor force, $8 \%$ of them enter the labor force in the following months, whereas, in the SIPP data, only $4 \%$ enter the labor force.

Starr (2014) examines the changes in added worker effect for the recessionary period of 2007-2009 using American Community Survey data. The probability of being employed in a given month for women increases by 4.4 percentage points from 2007 to 2009 if her husband is not employed. Estimates are not directly comparable to this current study findings since the study does not give us the estimate for the added worker effect. 
Parker and Skoufias (2004) find that the probability that a wife enters the labor in a given quarter increases by 16.2 percentage points during the Mexican economic recession in the 1990s. Although we expect the estimate to be up to three times as great as a monthly estimates, like ours, even after making an adjustment, this estimate of the added worker effect is much higher than the current study. Studies that estimate the added worker effect outside the United States are generally much higher (Fernandes \& Felício, 2005). Two facts can explain the overall high added worker effect in other countries. Liquidity constraints are substantially more important for households in these countries, and thus the responsiveness of wives' entry into the labor market to family income shocks is likely. Another explanation is that the husband's unemployment is primarily permanent, and as a result, wives act proactively to the husbands' job loss in these countries.

Gong (2011) estimates that females increase their full-time employment in a given month by 4 percentage points after observing their partner's job loss, based on a period of a mild recession in Australia. However, the measure is not precise, and there is a potential bias in estimates of the added worker effect since the author measures the partner's job loss during the previous 12 months. On the other hand, the current study finds an immediate labor force response of a wife where her husband is unemployed in the immediate past month.

Using a measure of wife's participation based on monthly data, Fernandes and Felício (2005) estimate an added worker effect of 10 percentage points for Brazil, stronger than studies from the United States. The study measures wife's transition probability from inactivity to activity, where inactivity includes wives who are both unemployed and not in the labor force. The economic structure of Brazil is different from that of the United States. 
Wife's labor force participation in Brazil was about 40 percent, and in the United States, the wife's labor force participation was 75 percent. As noted above, responses in less developed economies like Brazil may be stronger because workers face of greater capital constraints.

\section{Conclusion}

A married woman's choice of labor supply is determined by a variety of factors. There are factors that arise from general economic conditions (economic recession or prosperity). Social policies (for example, the Supplemental Nutrition Assistance Program, Unemployment Insurance) also influence her choice of whether to work or not. Family or household characteristics also contribute to her labor supply choice. Analysis of the added worker effect is important as it provides family insurance along with other contributions to the economy.

From the current analysis we observe that there are factors that contribute to wives' choice of labor force participation and labor supply. Wives are more likely to participate in the labor force when their husbands suffer a job loss. A woman's chance of participating in the labor force increases by 1.5 percentage points when her husband is unemployed. I also find significant contributions of husbands' age and education, wives' education, husbands' and wives' ages, and race and ethnicity in explaining wives' labor supply. Number of children is also a good predictor of wives' labor force participation. Local economic activity as measured by the state unemployment rate also contributes to wives' labor force participation.

The analysis based on SIPP gives us important findings as it tracks people on a monthly basis for a long period compared to other available national surveys. Due to its 
four-month recall period, information on labor supply is more reliable than that available in other studies. A shorter recall period improves our analysis. SIPP is one of the few datasets with monthly data on a large sample. In the added worker literature, the present findings add up to a new line of research. Few studies have looked at the added worker effect during the Great Recession. This paper shows that the added worker effect exists in a month-by-month analysis, although it is of modest size, and may vary across months. It may be the case that getting a job is hard for some wives to enter the labor force when their husbands have recently lost a job, or that the wife doesn't search for a job as the family has other income sources (Gong, 2011; Lundberg, 1985). Husband's employment status does predict wife's choice of labor force entry most clearly during the recession. Husband's duration of unemployment is unable to predict wife's labor force choice. 


\section{References}

Blau, F. D., \& Winkler, A. E. (2017). The Economics of Women, Men, and Work. Oxford University Press.

Cameron, A. C., \& Trivedi, P. K. (2010). Microeconometrics using Stata. Stata Press.

Cotter, D. A., Hermsen, J. M., \& Vanneman, R. (2004). Gender inequality at work. Russell Sage Foundation New York.

Cullen, J. B., \& Gruber, J. (2000). Does unemployment insurance crowd out spousal labor supply? Journal of Labor Economics, 18(3), 546-572.

Currie, J. (2003). US food and nutrition programs. In Means-tested transfer programs in the United States (pp. 199-290). University of Chicago Press.

Czajka, J. L., Mabli, J., \& Cody, S. (2008). Sample loss and survey bias in estimates of Social Security beneficiaries: A tale of two surveys. Washington, DC: Mathematica Policy Research, Inc.

Dynarski, M., \& Sheffrin, S. M. (1987). Consumption and unemployment. The Quarterly Journal of Economics, 102(2), 411-428.

Fernandes, R., \& Felício, F. de. (2005). The entry of the wife into the labor force in response to the husband's unemployment: A study of the added worker effect in Brazilian Metropolitan areas. Economic Development and Cultural Change, 53(4), 887-911.

Ghignoni, E., \& Verashchagina, A. (2016). Added worker effect during the Great Recession: Evidence from Italy. International Journal of Manpower, 37(8), 1264 1285. 
Gong, X. (2011). The added worker effect for married women in Australia. Economic Record, 87(278), 414-426.

Hoynes, H. W., \& Schanzenbach, D. W. (2012). Work incentives and the food stamp program. Journal of Public Economics, 96(1-2), 151-162.

Karaoglan, D., \& Okten, C. (2015). Labor-Force Participation of Married Women in Turkey: A Study of the Added-Worker Effect and the Discouraged-Worker Effect. Emerging Markets Finance and Trade, 51(1), 274-290.

Lundberg, S. (1985). The added worker effect. Journal of Labor Economics, 3(1, Part 1), $11-37$.

Mankart, J., \& Oikonomou, R. (2016). The rise of the added worker effect. Economics Letters, 143, 48-51.

Mattingly, M. J., \& Smith, K. E. (2010). Changes in Wives’ Employment When Husbands Stop Working: A Recession-Prosperity Comparison. Family Relations, 59(4), 343-357.

Mincer, J. (1962). Labor force participation of married women: A study of labor supply. In Aspects of labor economics (pp. 63-105). Princeton University Press.

Moffitt, R. (2002). Welfare programs and labor supply. Handbook of Public Economics, $4,2393-2430$.

Moffitt, R. (2015). The US safety net and work incentives: The great recession and beyond. Journal of Policy Analysis and Management, 34(2), 458-466.

Parker, S. W., \& Skoufias, E. (2004). The added worker effect over the business cycle: Evidence from urban Mexico. Applied Economics Letters, 11(10), 625-630. 
SIPP 2008. (n.d.). Retrieved August 20, 2019, from https://www2.census.gov/programssurveys/sipp/tech-documentation/complete-documents/2008/sipp-2008-panelwave-01-core-file.pdf

Smith, K. E., \& Mattingly, M. J. (2014). Husbands’ Jobs Loss and Wives' Labor Force Participation during Economic Downturns: Are All Recessions the Same. Monthly Lab. Rev., 137, 1.

SNAP Eligibility | USDA-FNS. (n.d.). Retrieved November 21, 2019, from https://www.fns.usda.gov/snap/recipient/eligibility

Spletzer, J. R. (1997). Reexamining the added worker effect. Economic Inquiry, 35(2), $417-427$.

Starr, M. A. (2014). Gender, added-worker effects, and the 2007-2009 recession: Looking within the household. Review of Economics of the Household, 12(2), 209-235.

The Personal Responsibility and Work Opportunity Reconciliation Act of 1996. (2015, November 23). ASPE. https://aspe.hhs.gov/report/personal-responsibility-andwork-opportunity-reconciliation-act-1996

US Business Cycle Expansions and Contractions. (2010, September 20). https://www.nber.org/cycles.html

U.S. Census Bureau. (n.d.). SIPP Introduction \& History. Retrieved October 8, 2019, from https://www.census.gov/programs-surveys/sipp/about/sipp-introductionhistory.html

Whdfs28.pdf. (n.d.). Retrieved August 28, 2019, from https://www.dol.gov/whd/regs/compliance/whdfs28.pdf 
Woytinsky, W. S. (1940). Additional Workers and the Volume of Unemployment in the Depression. Committee on Social Security, Social Science Research Council. 


\section{Appendix}

\section{$\underline{\text { Appendix A }}$}

\section{Female labor force participation and unemployment}

Labor supply and work incentives have long been a central concern in economic research. Up through the middle of the $20^{\text {th }}$ century, women were perceived as secondary earners within the family and mostly dependent on husbands' earnings. Women were primarily responsible for household activities. A married woman's choice of labor supply has attracted attention as their participation has been increasing and the gap between male and female labor force participation is decreasing (Blau \& Winkler, 2017). Despite sharing other responsibilities, woman still bears a larger share of household and childcare responsibilities than a man does. Nonetheless, the labor supply elasticity of women has declined significantly and has approached that of men (Blau \& Winkler, 2017).

The rapid growth of women's labor force participation is one of the most significant social and economic developments after the Second World War. ${ }^{6}$ At the beginning of the $20^{\text {th }}$ century, only 20 percent of women were in the labor force, whereas, by 2000 , almost 60 percent of all women were in the labor force. This dramatic shift can be explained by the underlying three facts (Blau \& Winkler, 2017; Cahuc, Carcillo, \& Zylberberg, 2014). First, the transformation of gender roles in society. Earlier, by tradition, women were engaged with household activities including raising children. As a result of industrialization, household activities become simple. Men are also increasingly participating in household activities. This gives wives more time to work outside the

\footnotetext{
${ }^{6}$ The labor force participation rate of a particular group, here 25-54 years, is defined by the number of its members who are in the labor force divided by the total number of the people in that group. This study deals with couple of 25 to 54 years and detail sample selection process are explained in section 6 .
} 
home. Second, families have started depending on female earnings, and we see an increasing number of female headed households also. Men as a single earner are no longer sufficient to meet the families' perceived needs. Income source by husband and wife is crucial. Due to women's increased labor force participation, the material wellbeing of the families have increased significantly. Women's labor force participation changed the usual family structure. Third, we see a decreasing fertility associated with industrialization, known as "fertility transition." This results in smaller family size, and more room for wives to work outside.

Cross-country studies identified factors that are responsible for the increased labor force participation for women in many countries. First, there are studies that show that there is an impact of children on the working decisions of women. Bloom et al. (2009) studied the effect of fertility on labor force participation by women, estimating that the birth of a baby reduces women's labor supply in her reproductive age by 2 years. Second, female labor force participation is accelerated by the ability to control birth timing of couples. Bailey (2006) shows that access to birth control pills significantly decreases the probability of first birth by 14 to 18 percent and increases labor supply by females. Third, increased childcare facilities may motivated women to join the labor force. We see an increasing female employment in Scandinavian countries and Canada in response to the growth in the availability of child care. 
Figure 1. Labor Force Participation Rates of Females and Males (age 25-54), 2000-2019

$(\%)$

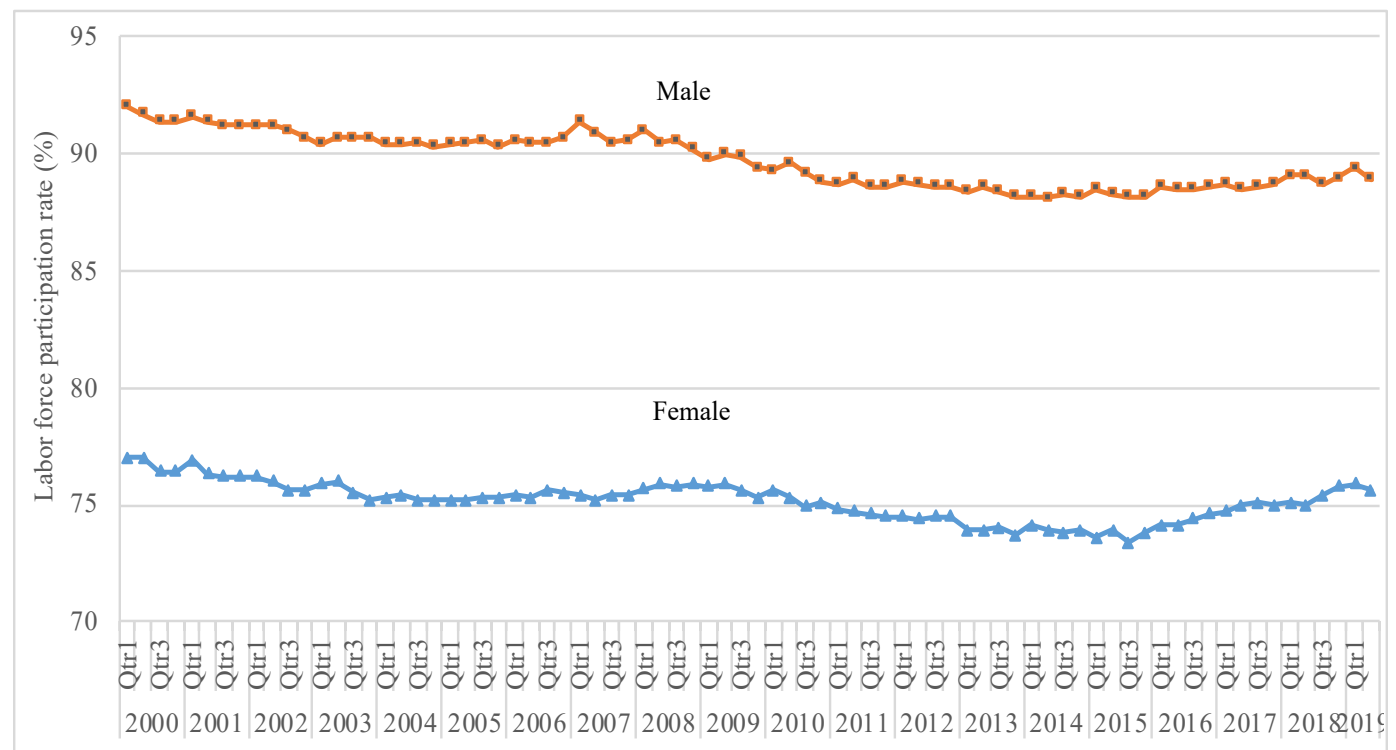

Source: Labor Force Statistics from the Current Population Survey available at www.bls.gov Note: Based on the civilian population age 25 to 54 .

Figure 1 shows the quarterly labor force participation in the U.S. from 2000 to 2019 by gender for the age group 25 to $54 .^{7}$ Over this period we see a decline in labor force participation for males. Female labor force participation remains relatively stable. In particular, during the Great Recession, we see a decline in labor force participation for males of about one percentage point between December 2007 and June $2009 .{ }^{8}$ However, the labor force participation rate for females remains at the same level over this period. Considering the overall trend, we can infer that due to the Great Recession, males are less likely to participate in the labor market whereas females are more likely to be in the labor market. It seems likely that women are playing a greater role in supporting their families.

\footnotetext{
${ }^{7}$ According to the BLS, “...the CPS defines the labor force to include all individuals 16 years of age and over who worked for pay or profit during the reference week or actively sought paid employment during the 4 weeks prior to the reference week".

${ }^{8}$ The Great Recession officially starts in December 2007 and ends in June 2009, with a duration of 18 months. However, the adverse impacts of the Great Recession continues even after the official end period. Figure 8 shows there was a rise of unemployment from December 2007 and continues to rise even after the official end of Great Recession. The unemployment hovered around 10 percent after the Great Recession.
} 
The growth in female labor force participation that has occurred during the Great Recession should be considered in the context of the patterns of women's employment over the life cycle. This is illustrated in figure 2 for five different years, before and after the Great Recession. According to the figure, women in the age group of 40 to 49 participated in the labor market at the highest levels. After that, it follows a sharp decline for the 50 to 54 age group. Women in the age group 40 to 49 , older married women with school-age or grown children, entered or reenter the labor force in increasing numbers. In contrast, change occurs for those between the ages 25 to 35, who may have preschooler children and need to stay at home for family care.

Figure 2. Labor Force Participation Rates of Females by Age, 2000-2019 (\%)

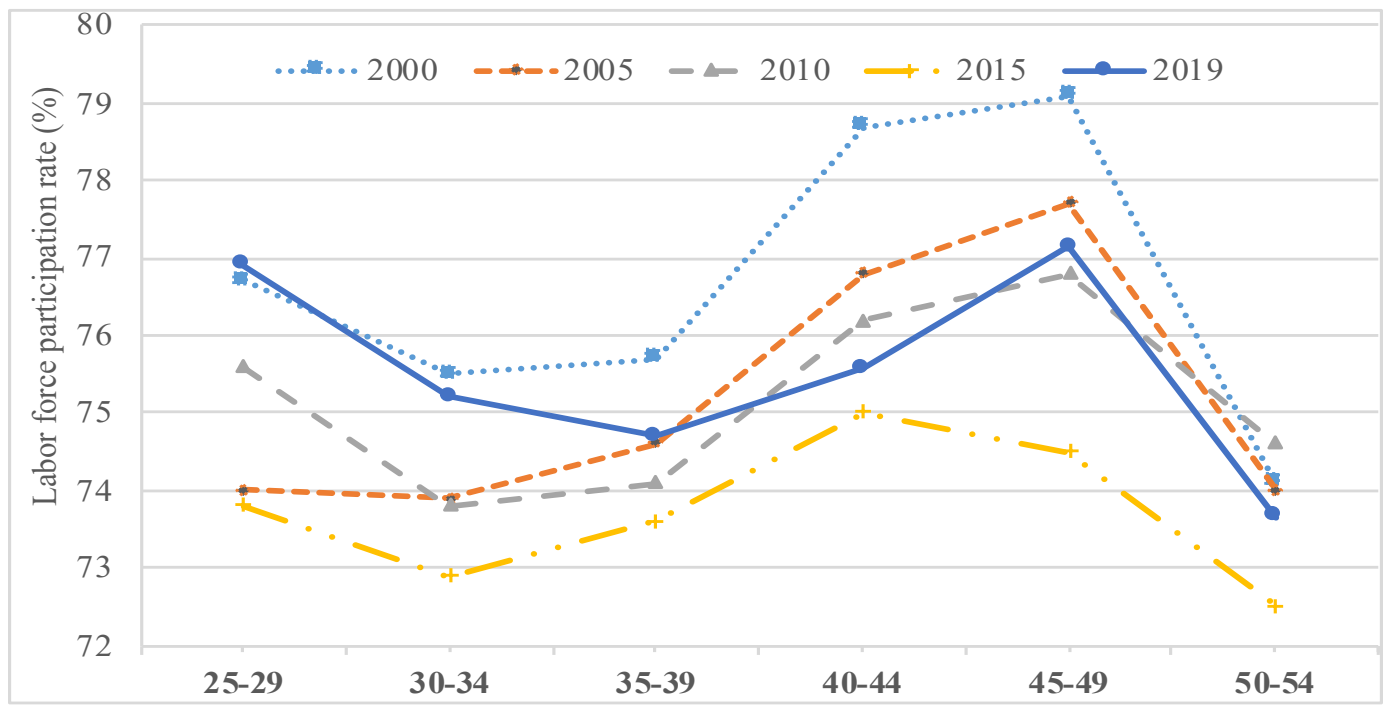

Source: Labor Force Statistics from the Current Population Survey available at www.bls.gov Note: Based on the civilian population age 25 to 54. Data for 2919 is available for the first seventh months.

Of the years considered, we see the highest percentage of the females participated in the labor market in 2000. Conversely, after the recession, in 2010 or 2015, we see a lower percentage of female participation in the labor market. Figure 3 shows the labor force participation by race and gender during the past two decades. Overall, participation 
declined for both black and white males. Black males faced a sharp decline in participation during the recessionary period compared to white males. In the same way, black females faced a sharp decline in labor force participation relative to white females. This gives us the intuitive conclusion that people face racial challenges in obtaining jobs during the recessionary period when the number of available jobs is reduced. White males and females enjoy relatively better positions during the recessionary period than black males and females.

Figure 3. Labor Force Participation Rates for Females and Males by Race (age 25-54), 2000-2019 (\%)

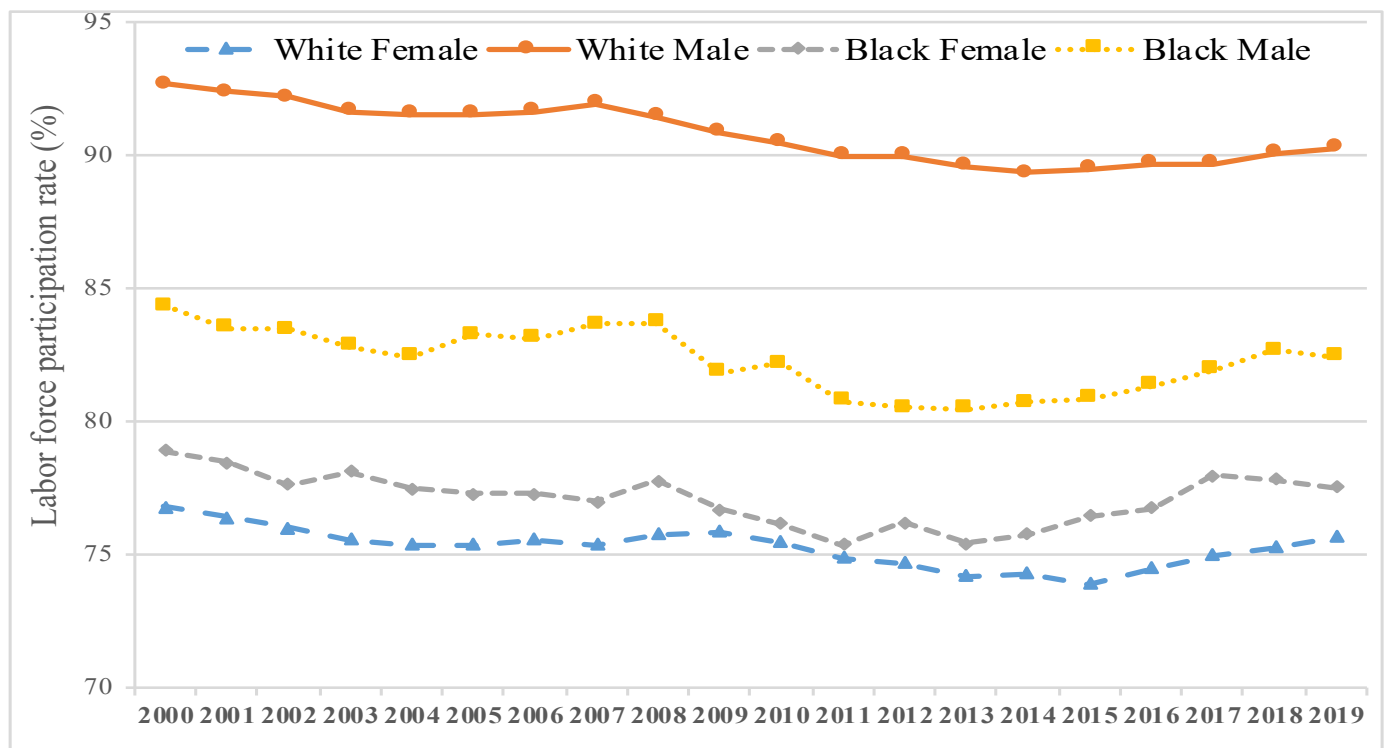

Source: Labor Force Statistics from the Current Population Survey available at www.bls.gov Note: Based on the civilian population age 25 to 54 .

The unemployment rates of women and men follow different patterns over the period for the age group 25 to 54 and this is depicted in figure 4. During the recession, males lost their jobs at a higher rate than females. This can be explained by the types of jobs that men and women had, i.e., gender differences in occupations and industries 
(Michaelides \& Mueser, 2013). Males were overrepresented in construction and manufacturing, whereas, women were more likely to be in relatively secure jobs such as those in education and health services.

Figure 4. Unemployment Rates for Females and Males, 2000-2019 (\%)

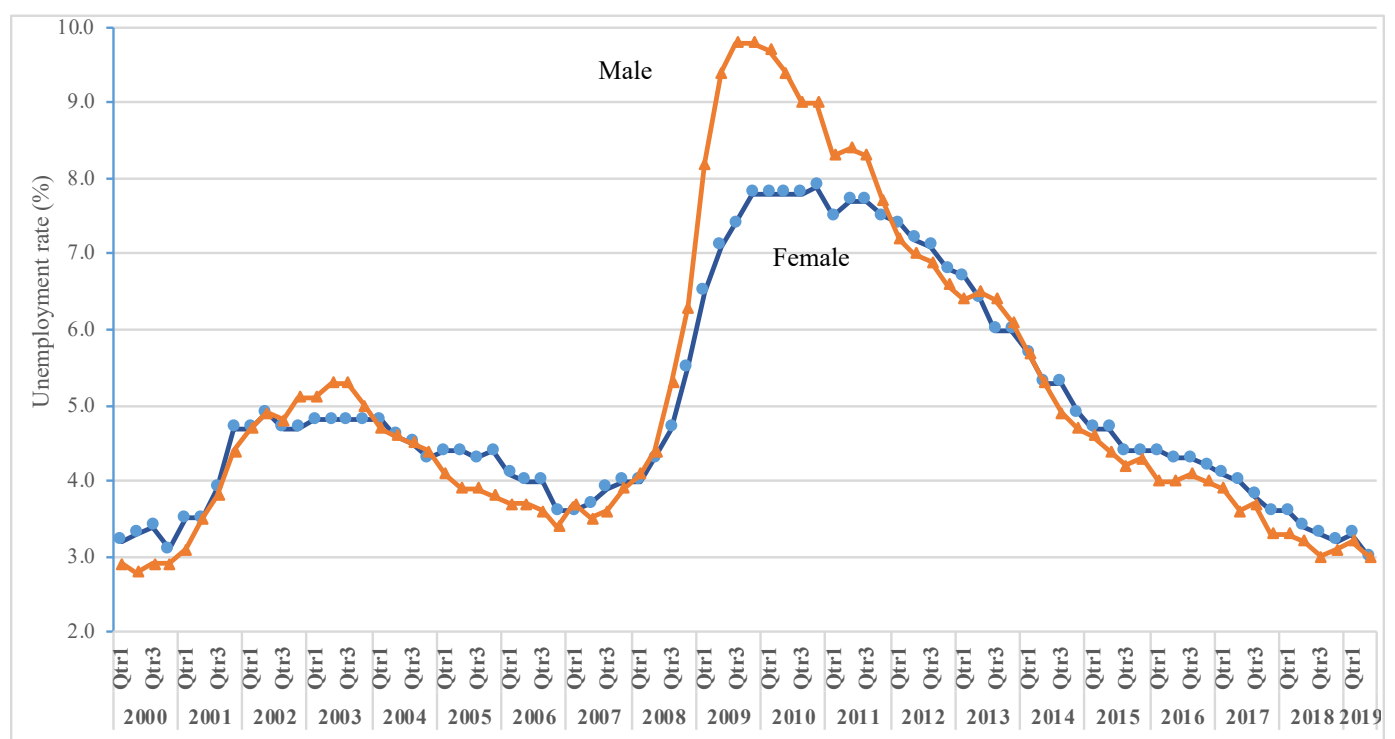

Source: Labor Force Statistics from the Current Population Survey available at www.bls.gov Note: Based on the civilian population age 25 to 54 .

Also, men are heavily represented in blue-collar jobs (Blau \& Winkler, 2017). In these jobs there are high frequencies of layoffs and greater unemployment as a result. Women are more likely to be employed in white-collar jobs, which experience lower layoff and lower unemployment. However, service sector jobs are disproportionately filled by females and have above average unemployment.

The racial profile for the unemployment rate for the age group 25 to 54 by race is illustrated in figure 5. During this period, whites and Asians experienced similar patterns in unemployment rate. However, Black males and black females are different from others. Asians are relatively better off over this period. Asians are highly skilled 
immigrants and engage in relatively secure jobs, and so they are less affected by the Great Recession. Conversely, black males and black females are strongly affected by the Great Recession. During the recessionary period, black males had an unemployment rate of about 15 to 20 percent as they are more likely to be in less skilled or informal jobs.

Figure 5. Unemployment Rates of Female and Male by Race (25-54), 2000-2019 (\%)

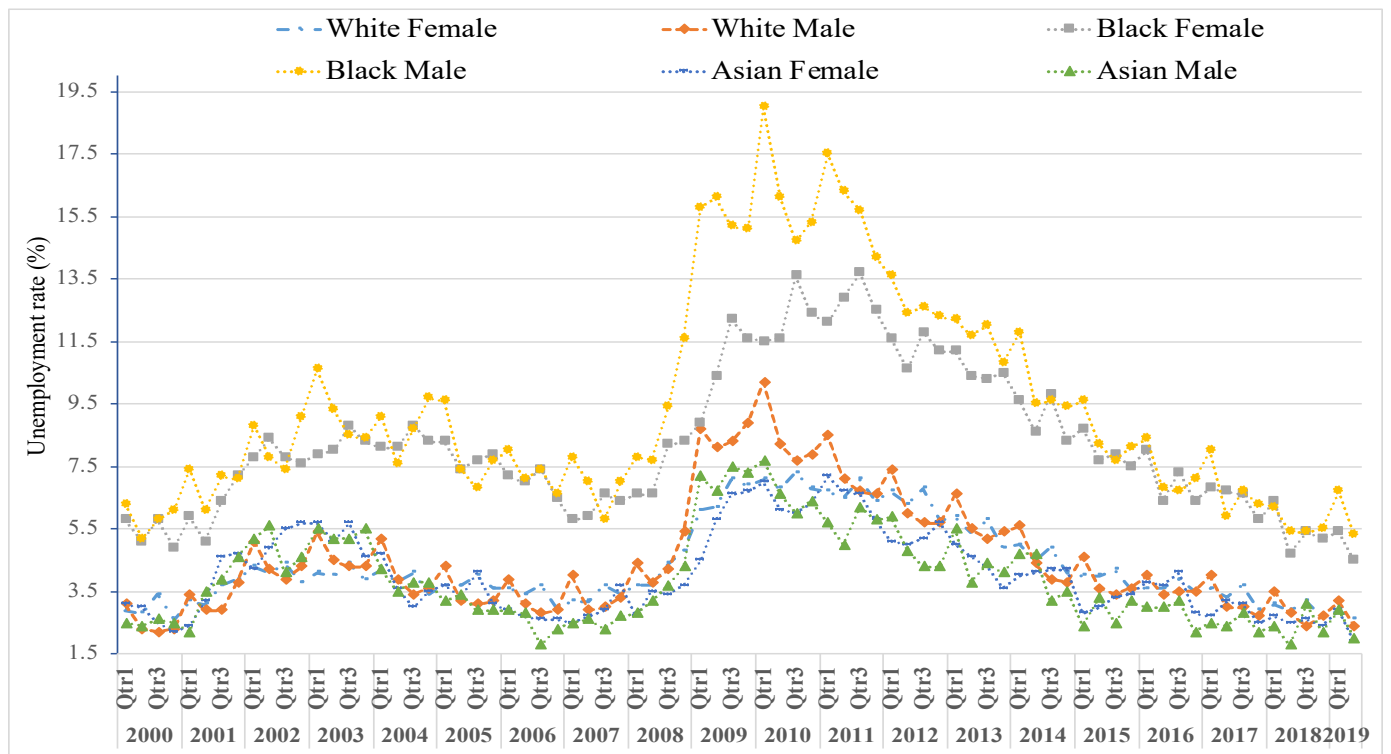

Source: Labor Force Statistics from the Current Population Survey available at www.bls.gov Note: Based on the civilian population age 25 to 54.

There are differences in labor force participation and unemployment rate by race and gender, and although the ranking of differences remain much the same, levels of unemployment are very different during the recession than periods of normal economic activity. Different groups of people are disproportionately affected by job loss in the Great Recession. Job loss by females directly affects her labor supply in the market. Likewise, job loss by a married man also affects his wife's labor supply. The change in labor supply by a wife depends on whether she was already in the labor market or not. It could be a good exercise for us to investigate how wife's labor supply was affected by 
husband's job loss and other factors in the Great Recession. It is also important to look at the changes for short time (e. g., monthly) along with longer time (e. g., annually or more). 


\section{$\underline{\text { Appendix B }}$}

Table A1. Odds ratios for logit predicting wives' likelihood of labor force participation at $t$ as function of husband's labor force status in prior month, 2008.

\begin{tabular}{|c|c|c|c|c|c|c|c|c|c|c|}
\hline & $\begin{array}{l}\text { (1) } \\
\text { Aug: wife } \\
\text { in lf in (t- } \\
\text { l) }\end{array}$ & $\begin{array}{c}\text { (2) } \\
\text { Sept: } \\
\text { wife in lf } \\
\text { in }(t-1)\end{array}$ & $\begin{array}{l}\text { (3) } \\
\text { Oct: wife } \\
\text { in lf in (t- } \\
\text { 1) }\end{array}$ & $\begin{array}{l}\text { (4) } \\
\text { Nov: wife } \\
\text { in If in (t- } \\
\text { l) }\end{array}$ & $\begin{array}{c}\text { (5) } \\
\text { Dec: } \\
\text { wife in lf } \\
\text { in }(t-1)\end{array}$ & $\begin{array}{c}\text { (6) } \\
\text { Aug: } \\
\text { wife not } \\
\text { in lf in (t- } \\
\text { 1) }\end{array}$ & $\begin{array}{c}\text { (7) } \\
\text { Sept: } \\
\text { wife not } \\
\text { in lf in (t- } \\
\text { 1) }\end{array}$ & $\begin{array}{c}\text { (8) } \\
\text { Oct: } \\
\text { wife not } \\
\text { in lf in (t- } \\
\text { 1) }\end{array}$ & $\begin{array}{c}\text { (9) } \\
\text { Nov: } \\
\text { wife not in } \\
\text { lf in }(t-1)\end{array}$ & $\begin{array}{c}\text { (10) } \\
\text { Dec: } \\
\text { wife not } \\
\text { in lf in ( } t- \\
\text { 1) }\end{array}$ \\
\hline \multicolumn{11}{|l|}{ 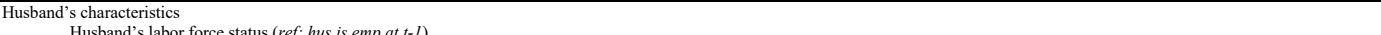 } \\
\hline $\begin{array}{l}\text { Husband's labor force status (ref: hus is emp at } t-I) \\
\text { Husband is unemployed at }(\mathrm{t}-1)\end{array}$ & 0.593 & 0.532 & $0.434 * *$ & & 2976 & & & 0,619 & & 189 \\
\hline Husband not in labor force at (t-1) & $\begin{array}{c}(0.397) \\
2.942 \\
(2.993)\end{array}$ & $\begin{array}{c}(0.244) \\
0.587 \\
(0.245\end{array}$ & $\begin{array}{c}(0.169) \\
0.755 \\
(0.488)\end{array}$ & $\begin{array}{c}10.708) \\
0.906 \\
(0.432)\end{array}$ & $\begin{array}{l}(2.503) \\
2.964 \\
(2.243)\end{array}$ & $\begin{array}{c}(0.632) \\
1.137 \\
(0.598)\end{array}$ & $\begin{array}{c}(0.391) \\
1.054 \\
(0.353)\end{array}$ & $\begin{array}{c}(0.373) \\
1.273 \\
(0.459)\end{array}$ & $\begin{array}{c}10.711) \\
0.601 \\
(0.344)\end{array}$ & $\begin{array}{l}1.189 \\
(0.521) \\
1.821 * \\
(0.655)\end{array}$ \\
\hline \multicolumn{11}{|l|}{ Husband's education (ref: some college) } \\
\hline Less than high school & $\begin{array}{c}1.211 \\
(0.653)\end{array}$ & $\begin{array}{c}0.773 \\
(0.344)\end{array}$ & $\begin{array}{c}0.777 \\
(0.422)\end{array}$ & $\begin{array}{c}0.676 \\
(0.211)\end{array}$ & $\begin{array}{c}0.842 \\
(0.431)\end{array}$ & $\begin{array}{c}1.015 \\
(0.593)\end{array}$ & $\begin{array}{c}0.806 \\
(0.315)\end{array}$ & $\begin{array}{c}1.206 \\
(0.380)\end{array}$ & $\begin{array}{c}0.810 \\
(0.263)\end{array}$ & $\begin{array}{c}0.812 \\
(0.268)\end{array}$ \\
\hline High school graduate & $\begin{array}{c}0.714 \\
(0.311)\end{array}$ & $\begin{array}{l}1.172 \\
(0.376)\end{array}$ & $\begin{array}{l}1.277 \\
(0.372)\end{array}$ & $\begin{array}{c}0.645 \\
(0.176)\end{array}$ & $\begin{array}{c}0.615 \\
(0.183)\end{array}$ & $\begin{array}{l}1.410 \\
(0.586)\end{array}$ & $\begin{array}{l}1.015 \\
(0.260)\end{array}$ & $\begin{array}{l}1.296 \\
(0.331)\end{array}$ & $\begin{array}{l}1.104 \\
(0.345)\end{array}$ & $\begin{array}{c}1.313 \\
(0.399)\end{array}$ \\
\hline Bachelor degree & 1.743 & 1.772 & 1.376 & 0.763 & 1.604 & 0.757 & 0.903 & 0.623 & 0.691 & 0.806 \\
\hline & $(0.919)$ & $(0.682)$ & $(0.521)$ & $(0.280)$ & $(0.587)$ & $(0.289)$ & $(0.216)$ & $(0.192)$ & $(0.261)$ & $(0.294)$ \\
\hline Masters or higher & $\begin{array}{c}0.657 \\
(0.403)\end{array}$ & $\begin{array}{r}1.701 \\
(0.889)\end{array}$ & $\begin{array}{r}1.741 \\
(1.182)\end{array}$ & $\begin{array}{r}1.311 \\
(0.720)\end{array}$ & $\begin{array}{l}1.974 \\
(1.086)\end{array}$ & $\begin{array}{l}0.409 * * \\
(0.165)\end{array}$ & $\begin{array}{c}0.365 * * * \\
(0.123)\end{array}$ & $\begin{array}{l}0.550^{* *} \\
(0.162)\end{array}$ & $\begin{array}{c}1.131 \\
(0.377)\end{array}$ & $\begin{array}{c}0.553 \\
(0.279)\end{array}$ \\
\hline Husband's age & $\begin{array}{c}0.956 \\
(0.044)\end{array}$ & $\begin{array}{l}1.026 \\
(0.029)\end{array}$ & $\begin{array}{c}0.985 \\
(0.038)\end{array}$ & $\begin{array}{c}0.978 \\
(0.029)\end{array}$ & $\begin{array}{c}0.994 \\
(0.032)\end{array}$ & $\begin{array}{c}0.994 \\
(0.041)\end{array}$ & 1.012 & 1.007 & 1.012 & 1.005 \\
\hline Husband's unemployment insurance (ref: no ins.) & $\begin{array}{c}(0.044) \\
\ldots\end{array}$ & $\begin{array}{c}(.0276) \\
1.376 \\
(1.068)\end{array}$ & $\begin{array}{c}0.030) \\
3.180 \\
(3.185)\end{array}$ & $\begin{array}{c}0.632 \\
0.470)\end{array}$ & $\begin{array}{c}0.537 \\
0.399)\end{array}$ & $\begin{array}{c}(0.041) \\
\cdots\end{array}$ & $\begin{array}{c}2.096 \\
(1.603)\end{array}$ & $\begin{array}{c}0.961 \\
(1.075)\end{array}$ & $\begin{array}{c}(0.0210 \\
0.210 \\
(0.221)\end{array}$ & $\begin{array}{l}2.0254 \\
(1.454)\end{array}$ \\
\hline \multirow{2}{*}{\multicolumn{11}{|c|}{$\begin{array}{l}\text { Wife's characteristics } \\
\text { Wife's education (ref: }\end{array}$}} \\
\hline & & & & & & & & & & \\
\hline Less than high school & $\begin{array}{c}0.538 \\
(0.257)\end{array}$ & $\begin{array}{c}1.558 \\
(0.959)\end{array}$ & $\begin{array}{c}0.876 \\
(0.506)\end{array}$ & $\begin{array}{c}0.674 \\
(0.227)\end{array}$ & $\begin{array}{c}0.490 \\
(0.221)\end{array}$ & $\begin{array}{c}0.416 \\
(0.246)\end{array}$ & $\begin{array}{c}1.067 \\
(0.430)\end{array}$ & $\begin{array}{c}0.452^{* *} \\
(0.164)\end{array}$ & $\begin{array}{c}0.679 \\
(0.278)\end{array}$ & $\begin{array}{c}1.342 \\
(0.441)\end{array}$ \\
\hline High school graduate & $\begin{array}{l}1.119 \\
(0.557)\end{array}$ & $\begin{array}{c}0.834 \\
(0.274)\end{array}$ & $\begin{array}{c}0.703 \\
(0.224)\end{array}$ & $\begin{array}{c}0.782 \\
(0.221)\end{array}$ & $\begin{array}{c}0.943 \\
(0.342)\end{array}$ & $\begin{array}{l}0.463^{*} \\
(0.214)\end{array}$ & $\begin{array}{l}1.051 \\
(0.252)\end{array}$ & $\begin{array}{l}0.528^{* *} \\
(0.148)\end{array}$ & $\begin{array}{l}1.140 \\
(0.293)\end{array}$ & $\begin{array}{c}1.212 \\
(0.340)\end{array}$ \\
\hline \multirow[t]{2}{*}{ Bachelor degree } & 0.821 & 1.040 & 1.087 & $\begin{array}{l}1.215 \\
(0.348)\end{array}$ & $\begin{array}{l}0.553^{*} \\
(0.196)\end{array}$ & $\begin{array}{r}1.647 \\
(0.624)\end{array}$ & $1.950^{* * * *}$ & $\begin{array}{l}1.360 \\
(0.418)\end{array}$ & 1.162 & 1.622 \\
\hline & $\begin{array}{c}(0.393) \\
0.721\end{array}$ & $\begin{array}{c}(0.371) \\
0.818\end{array}$ & $\begin{array}{c}(0.323) \\
0.815\end{array}$ & $\begin{array}{c}(0.348) \\
2.170\end{array}$ & $\begin{array}{c}(0.196) \\
1.630\end{array}$ & $\begin{array}{l}(0.624) \\
6.660^{* * *}\end{array}$ & $\begin{array}{l}(0.460) \\
2.103 * *\end{array}$ & $\begin{array}{l}(0.418) \\
1.926^{*}\end{array}$ & $\begin{array}{c}(0.403) \\
0.689\end{array}$ & $\begin{array}{c}(0.521) \\
1.038\end{array}$ \\
\hline Masters or higher & $(0.443)$ & $(0.331)$ & $(0.349)$ & $(1.199)$ & $(0.957)$ & $(2.612)$ & $(0.679)$ & $(0.645)$ & $(0.350)$ & $(0.575)$ \\
\hline Wife's age & 1.109 & $1.376^{*}$ & 1.303 & 1.202 & $1.359^{*}$ & 1.286 & $1.290^{* *}$ & 1.218 & 1.159 & 0.856 \\
\hline Wife's age square ${ }^{(a)}$ & $\begin{array}{c}(0.291) \\
1.000 \\
(0.312)\end{array}$ & $\begin{array}{l}(0.232) \\
0.671^{*} \\
(0.148)\end{array}$ & $\begin{array}{c}(0.221) \\
0.745 \\
(0.148)\end{array}$ & $\begin{array}{c}(0.184) \\
0.832 \\
(0.160)\end{array}$ & $\begin{array}{c}(0.211) \\
0.684 * * \\
(0.131)\end{array}$ & $\begin{array}{c}(0.230) \\
0.722 \\
(0.165)\end{array}$ & $\begin{array}{c}(0.163) \\
0.685^{* *} \\
(0.112)\end{array}$ & $\begin{array}{l}(0.154) \\
0.751^{*} \\
(0.128)\end{array}$ & $\begin{array}{c}(0.152) \\
0.801 \\
(0.133)\end{array}$ & $\begin{array}{c}(0.107) \\
1.188 \\
(0.182)\end{array}$ \\
\hline \multicolumn{11}{|l|}{ Wife's race \& ethnicity (ref: White alone) } \\
\hline Black & $\begin{array}{c}0.649 \\
(0.391)\end{array}$ & $\begin{array}{c}0.569 \\
(0.212)\end{array}$ & $\begin{array}{c}1.090 \\
(0.461)\end{array}$ & $\begin{array}{c}1.074 \\
(0.437)\end{array}$ & $\begin{array}{c}0.324 * * * \\
(0.115)\end{array}$ & $\begin{array}{c}1.194 \\
(0.572)\end{array}$ & $\begin{array}{l}1.940^{* *} \\
(0.581)\end{array}$ & $\begin{array}{c}2.037 * * \\
(0.604)\end{array}$ & $\begin{array}{c}0.798 \\
(0.300)\end{array}$ & $\begin{array}{c}1.565 \\
(0.662)\end{array}$ \\
\hline Asian & $\begin{array}{c}3.730 \\
(3.768)\end{array}$ & $\begin{array}{l}0.458^{*} \\
(0.205)\end{array}$ & $\begin{array}{c}0.581 \\
(0.264)\end{array}$ & $\begin{array}{l}1.343 \\
(0.718)\end{array}$ & $\begin{array}{c}0.294 * * * \\
(0.111)\end{array}$ & $\begin{array}{l}0.233^{*} \\
(0.177)\end{array}$ & $\begin{array}{l}1.367 \\
(0.380)\end{array}$ & $\begin{array}{l}1.132 \\
(0.399)\end{array}$ & $\begin{array}{c}0.566 \\
(0.300)\end{array}$ & $\begin{array}{l}0.314^{*} \\
(0.187)\end{array}$ \\
\hline Other races or more than one & $\begin{array}{c}1.688 \\
(1.734)\end{array}$ & $\begin{array}{c}0.608 \\
(0.288)\end{array}$ & $\begin{array}{c}1.101 \\
(0.681)\end{array}$ & $\begin{array}{c}0.712 \\
(0.354)\end{array}$ & $\begin{array}{c}0.234 * * * \\
(0.116)\end{array}$ & $\begin{array}{c}1.266 \\
(0.916)\end{array}$ & $\begin{array}{c}0.782 \\
(0.392)\end{array}$ & $\begin{array}{c}0.930 \\
(0.624)\end{array}$ & $\begin{array}{l}1.123 \\
(0.637)\end{array}$ & $\begin{array}{c}0.507 \\
(0.385)\end{array}$ \\
\hline \multicolumn{11}{|l|}{$\begin{array}{l}\text { Family variables } \\
\text { Number of children under } 18 \text { (ref: } 2 \text { children) }\end{array}$} \\
\hline \multirow[t]{2}{*}{0 child } & 1.219 & 1.697 & 0.808 & 0.859 & 1.313 & 0.651 & $1.561 * *$ & 1.521 & 1.493 & 0.843 \\
\hline & $(0.545)$ & $(0.558)$ & $(0.278)$ & $(0.287)$ & & $(0.254)$ & & $(0.411)$ & $(0.483)$ & $(0.314)$ \\
\hline 1 child & $\begin{array}{l}1.087 \\
0.476\end{array}$ & $1.920^{* *}$ & $\begin{array}{c}0.869 \\
(0.349)\end{array}$ & $\begin{array}{c}0.820 \\
(0.236)\end{array}$ & $\begin{array}{l}0.489 * * \\
(0.170)\end{array}$ & $\begin{array}{c}0.710 \\
(0.273)\end{array}$ & $\begin{array}{c}1.299 \\
(0.290)\end{array}$ & $\begin{array}{l}0.576^{*} \\
(0.164)\end{array}$ & $\begin{array}{l}1.747 * * \\
(0.465)\end{array}$ & 0.953 \\
\hline 3 or more children & $\begin{array}{l}1.134 \\
(0.622)\end{array}$ & $\begin{array}{c}0.069 \\
(0.317)\end{array}$ & $\begin{array}{c}0.934 \\
(0.373)\end{array}$ & $\begin{array}{l}0.541^{*} \\
(0.177)\end{array}$ & $\begin{array}{c}0.596 \\
(0.241)\end{array}$ & $\begin{array}{c}0.673 \\
(0.234)\end{array}$ & $\begin{array}{l}1.105 \\
(0.238)\end{array}$ & $\begin{array}{c}0.773 \\
(0.158)\end{array}$ & $\begin{array}{c}0.652 \\
(0.191)\end{array}$ & $\begin{array}{c}0.893 \\
(0.256)\end{array}$ \\
\hline \multicolumn{11}{|l|}{ Residence (ref: metro) } \\
\hline Not metro & $\begin{array}{c}0.890 \\
(0.368)\end{array}$ & $\begin{array}{c}1.628 \\
(0.542)\end{array}$ & $\begin{array}{c}0.870 \\
(0.272)\end{array}$ & $\begin{array}{l}1.755^{*} \\
(0.568)\end{array}$ & $\begin{array}{c}1.086 \\
(0.331)\end{array}$ & $\begin{array}{c}1.057 \\
(0.350)\end{array}$ & $\begin{array}{c}1.067 \\
(0.286)\end{array}$ & $\begin{array}{c}1.253 \\
(0.281)\end{array}$ & $\begin{array}{c}0.682 \\
(0.207)\end{array}$ & $\begin{array}{c}1.038 \\
(0.303)\end{array}$ \\
\hline Not Identified & $\begin{array}{c}0.812 \\
(0.567)\end{array}$ & $\begin{array}{l}1.063 \\
(0.296)\end{array}$ & $\begin{array}{c}0.754 \\
(0.392)\end{array}$ & $\begin{array}{l}1.115 \\
(0.653)\end{array}$ & $\begin{array}{c}4.038 \\
(4.154)\end{array}$ & $\begin{array}{c}0.707 \\
(0.561)\end{array}$ & $\begin{array}{l}1.537 \\
(0.533)\end{array}$ & $\begin{array}{l}1.676 \\
(0.593)\end{array}$ & $\begin{array}{c}0.645 \\
(0.377)\end{array}$ & $\begin{array}{c}0.731 \\
(0.150)\end{array}$ \\
\hline \multirow[t]{2}{*}{ Unemployment (state) } & 1.203 & 0.894 & 0.984 & 0.897 & $0.851^{*}$ & 0.915 & 0.979 & $1.127^{*}$ & 1.050 & 0.947 \\
\hline & $(0.183)$ & $(0.096)$ & $\begin{array}{c}(0.096) \\
0.834\end{array}$ & $\begin{array}{c}(0.078) \\
8.962\end{array}$ & $\begin{array}{c}(0.073) \\
1.995\end{array}$ & $\begin{array}{c}(0.093) \\
0.001 * *\end{array}$ & $\begin{array}{c}(0.064) \\
0.001^{* * *}\end{array}$ & $\begin{array}{c}(0.079) \\
0.001^{* * *}\end{array}$ & $\begin{array}{c}(0.073) \\
0.002 * *\end{array}$ & $(0.078)$ \\
\hline \multirow[t]{2}{*}{ Constant } & $\begin{array}{c}7.479 \\
(34.840)\end{array}$ & $\begin{array}{c}0.111 \\
(0.364)\end{array}$ & $\begin{array}{c}0.834 \\
(2.494)\end{array}$ & $\begin{array}{c}8.962 \\
(25.790)\end{array}$ & $\begin{array}{c}1.995 \\
(5.630)\end{array}$ & $\begin{array}{c}0.001^{* *} \\
(0.003)\end{array}$ & $\begin{array}{c}0.001 * * * \\
(0.002)\end{array}$ & $\begin{array}{c}0.001^{* * *} \\
(0.002)\end{array}$ & $\begin{array}{l}0.002^{* * *} \\
(0.005)\end{array}$ & $\begin{array}{c}1.276 \\
(3.057)\end{array}$ \\
\hline & 6,193 & 8,168 & 8,105 & 7,997 & 7,860 & 2,308 & 2,974 & 2,794 & 2,686 & 2,617 \\
\hline
\end{tabular}

Notes: Standard errors in parentheses. ${ }^{* * *} \mathrm{p}<0.01,{ }^{*} * \mathrm{p}<0.05, * \mathrm{p}<0.1$

${ }^{(a)}$ Wife's age square is defined by age square divided by hundred. 
Table A2. Odds ratios for logit predicting wives' likelihood of labor force participation at $t$ as function of husband's labor force status in prior month, 2009 (wife in the labor force at $(t-1))$.

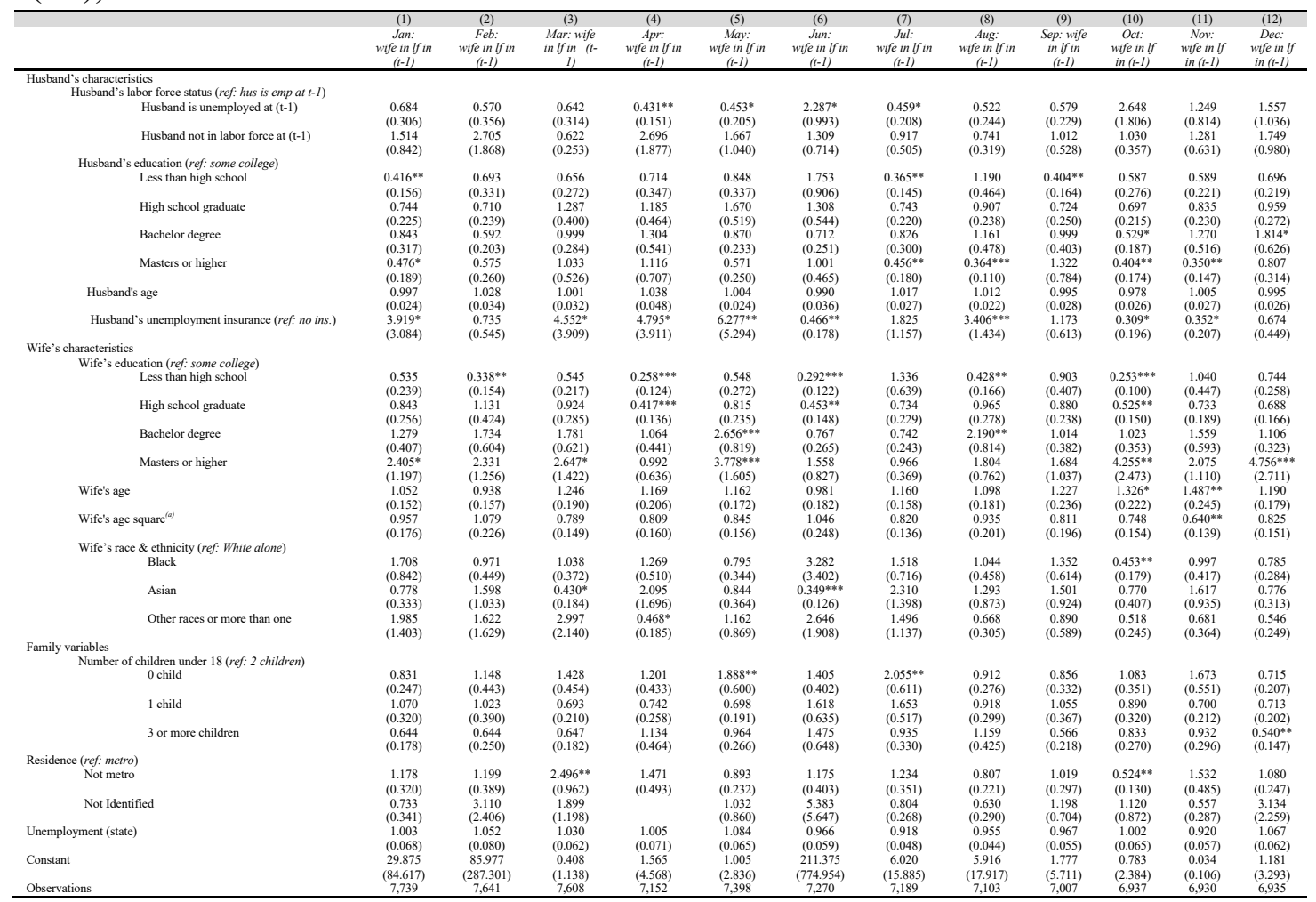

Notes: Standard errors in parentheses. $* * * \mathrm{p}<0.01, * * \mathrm{p}<0.05, * \mathrm{p}<0.1$

${ }^{(a)}$ Wife's age square is defined by age square divided by hundred. 
Table A3. Odds ratios for logit predicting wives' likelihood of labor force participation at $t$ as function of husband's labor force status in prior month, 2009 (wife not in the labor force at $(t-1))$.

\begin{tabular}{|c|c|c|c|c|c|c|c|c|c|c|c|c|}
\hline & $\begin{array}{c}\text { (1) } \\
\text { Jan: } \\
\text { wife ont in } \\
\text { lf in (t-1) }\end{array}$ & $\begin{array}{c}\text { Feb: } \\
\text { Feb } \\
\text { wife not } \\
\text { inf ly in } \\
\text { (t-1) }\end{array}$ & $\begin{array}{c}\text { (3) } \\
\text { Mar: } \\
\text { wife enot in } \\
\text { If in }(t-1)\end{array}$ & $\begin{array}{c}\text { (4) } \\
\text { Apr: } \\
\text { wife enot in } \\
\text { If in (t-1) }\end{array}$ & $\begin{array}{c}\text { (5) } \\
\text { May: } \\
\text { wife ont in } \\
\text { lf in (t-1) }\end{array}$ & $\begin{array}{c}\text { (6) } \\
\text { Jun: } \\
\text { wife en in } \\
\text { If in }(t-1)\end{array}$ & $\begin{array}{c}\text { (7) } \\
\text { Jul: wife } \\
\text { not in lf in } \\
(t-1)\end{array}$ & $\begin{array}{c}\text { (8) } \\
\text { Aug: wife } \\
\text { not in } 1 \text { if in } \\
(t-1)\end{array}$ & $\begin{array}{c}\text { (9) } \\
\text { Sep: } \\
\text { wife ont in } \\
\text { lf in (t-l) }\end{array}$ & $\begin{array}{c}\text { (10) } \\
\text { Oct: } \\
\text { wife in ot in } \\
\text { If in }(t-1)\end{array}$ & $\begin{array}{c}\text { (11) } \\
\text { Nov: } \\
\text { wife ont in } \\
\text { lf in (t-l) }\end{array}$ & $\begin{array}{c}\text { (12) } \\
\text { Dec: } \\
\text { wife ont in } \\
\text { If in (t-l) }\end{array}$ \\
\hline \multicolumn{13}{|l|}{$\begin{array}{l}\text { Husband's characteristics } \\
\text { Husband's labor force status (ref: hus is emp at } t-1)\end{array}$} \\
\hline 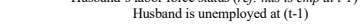 & 0.995 & 1.293 & 2.002 & $3.313 * *$ & 1.270 & $2.998^{*}$ & $2.952^{* * *}$ & 0.889 & 0.833 & 0.811 & $2.785^{* * *}$ & $2.863^{* * *}$ \\
\hline & $(0.460)$ & $(0.941)$ & $(0.845)$ & $(1.864)$ & $(0.691)$ & $(1.789)$ & $(1.436)$ & $(0.538)$ & $(0.617)$ & $(0.706)$ & $(1.232)$ & $(1.306)$ \\
\hline Husband not in labor force at $(\mathrm{t}-1)$ & $\begin{array}{l}1.062 \\
0.528\end{array}$ & $\begin{array}{l}0.315 \\
0256\end{array}$ & $\begin{array}{l}0.694 \\
0.386\end{array}$ & $2.089 *$ & $\begin{array}{l}0.777 \\
0.393\end{array}$ & 1.092 & 1.348 & $\begin{array}{l}2.001 \\
0.858\end{array}$ & 1.552 & $3.107 * *$ & 1.166 & $2.237 *$ \\
\hline Husband's education (ref: some college) & $(0.528)$ & $(0.256)$ & $(0.386)$ & $(0.824)$ & $(0.393)$ & $(0.509)$ & $(0.573)$ & $(0.858)$ & $(0.705)$ & (1.736) & $(0.611)$ & $(0.971)$ \\
\hline Less than high school & $2.498^{*}$ & 0.560 & 1.403 & 0.659 & 0.988 & 1.007 & $1.936^{* *}$ & 0.617 & 0.963 & 0.505 & 0.476 & 0.604 \\
\hline & $(1.227)$ & $(0.452)$ & $(0.569)$ & $(0.272)$ & $(0.523)$ & $(0.417)$ & $(0.624)$ & $(0.257)$ & $(0.371)$ & $(0.309)$ & $(0.227)$ & $(0.257)$ \\
\hline High school graduate & $\begin{array}{l}1.438 \\
0.997\end{array}$ & 1.061 & 0.710 & 1.267 & 0.573 & 0.971 & 1.428 & 0.944 & 0.664 & 0.949 & $\begin{array}{l}1.003 \\
0.0366\end{array}$ & 0.546 \\
\hline Bachelor degree & $\begin{array}{c}(0.497) \\
0.996\end{array}$ & $\left(\begin{array}{c}0.4032 \\
0.932\end{array}\right.$ & $\begin{array}{c}(0.239) \\
0.686\end{array}$ & $\begin{array}{l}(0.400) \\
0.653\end{array}$ & $\left(0.2066^{*}\right.$ & $\left(\begin{array}{l}0.3572 \\
0.572\end{array}\right.$ & $(0.515)$ & $(0.309)$ & $\begin{array}{c}(0.252) \\
0.601\end{array}$ & $\begin{array}{l}(0.374) \\
1506\end{array}$ & $(0.356)$ & $(0.205)$ \\
\hline Bachelor degree & $\begin{array}{l}0.996 \\
(0.358)\end{array}$ & $(0.357)$ & $(0.315)$ & $(0.264)$ & $\begin{array}{l}0.46^{\circ} \\
(0.197)\end{array}$ & $(0.238)$ & $(0.228)$ & $(0.159)$ & $(0.022)$ & $(0.561)$ & $(0.625)$ & $\begin{array}{l}0.422^{*} \\
(0200)\end{array}$ \\
\hline Masters or higher & $0.306^{* * *}$ & 0.398 & 1.334 & 0.670 & $0.328^{*}$ & $\begin{array}{c}(0.238) \\
0.563\end{array}$ & $0.426^{*}$ & $\begin{array}{l}0.159) \\
0.406 * *\end{array}$ & $\begin{array}{c}(0.222) \\
1.288\end{array}$ & $\begin{array}{c}(0.66) 1 \\
1.932\end{array}$ & $\left.0.456^{*}\right)$ & $\begin{array}{c}(0.200) \\
0.356\end{array}$ \\
\hline & $(0.146)$ & $(0.249)$ & $\begin{array}{l}1.354 \\
(0.507)\end{array}$ & $(0.296)$ & $\begin{array}{l}0.288^{2} \\
(0.192)\end{array}$ & $(0.269)$ & $\begin{array}{l}0.420^{\circ} \\
(0.194)\end{array}$ & $\begin{array}{l}0.400 \\
(0.140)\end{array}$ & $(0.510)$ & $\begin{array}{l}1.932 \\
(0.862)\end{array}$ & $(0.202)$ & $\begin{array}{l}0.350 \\
(0.230)\end{array}$ \\
\hline Husband's age & 0.983 & 0.970 & 0.993 & 0.999 & 0.988 & 1.039 & 1.001 & 0.987 & 0.984 & 1.042 & 1.002 & 0.988 \\
\hline & $(0.026)$ & $(0.019)$ & $(0.023)$ & $(0.026)$ & $(0.036)$ & $(0.033)$ & $(0.027)$ & $(0.024)$ & $(0.022)$ & $(0.042)$ & $(0.025)$ & $(0.027)$ \\
\hline Husband's unemployment insurance (ref: no ins.) & 1.220 & 0.601 & 1.519 & 0.488 & 0.976 & 0.603 & 0.856 & 1.424 & 1.594 & 0.333 & 0.412 & \\
\hline \multirow{2}{*}{\multicolumn{13}{|c|}{$\begin{array}{l}\text { Wife's characteristics } \\
\text { Wife's education (ref: some college) }\end{array}$}} \\
\hline & & & & & & & & & & & & \\
\hline $\begin{array}{l}\text { Wile seucutation (ref. isome college) } \\
\text { Less than high school }\end{array}$ & & & & & & & $0.437 * *$ & 0.835 & 0.874 & 1382 & 1318 & 1.024 \\
\hline & $(0.205)$ & $(0.588)$ & $(0.293)$ & $(0.231)$ & $(0.590)$ & $(0.390)$ & & $(0.345)$ & $(0.331)$ & $(0.834)$ & $(0.571)$ & $(0.024)$ \\
\hline High school graduate & 0.754 & 0.941 & 0.836 & $0.379 * * *$ & 1.194 & 0.903 & 0.774 & 1.007 & 0.846 & 1.072 & 1.014 & 1.275 \\
\hline & $(0.263)$ & $(0.359)$ & $(0.286)$ & $(0.127)$ & $(0.393)$ & $(0.317)$ & $(0.233)$ & $(0.389)$ & $(0.303)$ & $(0.436)$ & $(0.372)$ & $(0.450)$ \\
\hline Bachelor degree & 2.291 "** & $2.043 *$ & 0.916 & 1.288 & 1.119 & $1.866^{*}$ & 1.031 & 1.382 & 0.740 & 1.959 & 1.718 & 0.869 \\
\hline & $(0.796)$ & $(0.846)$ & $(0.347)$ & $(0.456)$ & $(0.513)$ & $(0.606)$ & $(0.389)$ & $(0.495)$ & $(0.252)$ & $(0.882)$ & $(0.701)$ & $(0.363)$ \\
\hline Masters or higher & 4.052**** & 1.712 & 0.884 & $\begin{array}{l}1.077 \\
0.597\end{array}$ & $\begin{array}{l}1.693 \\
0.872-200\end{array}$ & 1.806 & 0.739 & $2.781^{* * *}$ & $\begin{array}{l}1.056 \\
0.0433\end{array}$ & 0.615 & $3.206^{* * * *}$ & 1.034 \\
\hline Wife's age & ${ }_{0.688 * *}^{(1.674)}$ & $\begin{array}{c}(1.039) \\
1.302\end{array}$ & $\begin{array}{l}0.515 \%) \\
1399 *\end{array}$ & $\left(\begin{array}{ll}0.3971) \\
0911\end{array}\right.$ & $(0.872)$ & $\begin{array}{r}(0.890) \\
1309\end{array}$ & $\left(\begin{array}{l}0.461) \\
1050\end{array}\right.$ & $\begin{array}{l}(1.306) \\
1.333 *\end{array}$ & $(0.433)$ & $\begin{array}{c}(0.481) \\
1.173\end{array}$ & $\begin{array}{c}(1.1 .661) \\
1.171\end{array}$ & $\begin{array}{c}(0.781) \\
1.197\end{array}$ \\
\hline Wite's age & $\begin{array}{l}0.0887^{*} \\
(0.118)\end{array}$ & $\begin{array}{l}1.302 \\
(0.222)\end{array}$ & $\begin{array}{l}1.2969)^{\prime} \\
(0.267)\end{array}$ & $(0.135)$ & $\begin{array}{l}0.923 \\
(0.154)\end{array}$ & $\begin{array}{l}1.309 \\
(0.235)\end{array}$ & $\begin{array}{l}1.050 \\
(0.193)\end{array}$ & $(0.226)$ & $\begin{array}{l}0.927 \\
(0.138)\end{array}$ & $\begin{array}{l}1.173 \\
(0.239)\end{array}$ & $(0.208)$ & $(0.197)$ \\
\hline Wife's age square & $1.663^{* * *}$ & 0.745 & $0.637 *$ & 1.115 & 1.080 & $0.660^{*}$ & 0.899 & $0.690^{*}$ & 1.094 & 0.735 & 0.788 & 0.810 \\
\hline Wires age square & $\begin{array}{l}1.063374) \\
(0.374)\end{array}$ & $\begin{array}{l}0.143 \\
(0.162)\end{array}$ & $\begin{array}{l}0.3574 \\
(0.154)\end{array}$ & $(0.215)$ & $\begin{array}{l}1.080 \\
(0.218)\end{array}$ & $\begin{array}{l}0.000^{\circ} \\
(0.148)\end{array}$ & $(0.208)$ & $\begin{array}{l}0.00140 \\
(0.144)\end{array}$ & $(0.211)$ & $\begin{array}{l}0.138 \\
(0.188)\end{array}$ & $(0.180)$ & $\begin{array}{l}0.810 \\
(0.171)\end{array}$ \\
\hline $\begin{array}{l}\text { Wife's race \& ethnicity (ref: White alone) } \\
\text { Black }\end{array}$ & $2,147 *$ & & & & & & & & 1049 & & & \\
\hline & $(0.900)$ & $(1.355)$ & $(0.790)$ & $(0.785)$ & (0.577) & $\begin{array}{l}1.149 \\
(0.502)\end{array}$ & $(0.575)$ & $(0.300)$ & $(0.516)$ & $(0.613)$ & $(0.586)$ & $(0.955)$ \\
\hline Asian & 0.626 & 1.569 & $0.200^{* * *}$ & 0.986 & 0.924 & 0.529 & 1.572 & 0.483 & 0.883 & $0.124^{*}$ & $0.193^{* *}$ & 0.996 \\
\hline & $(0.298)$ & $(0.729)$ & $(0.147)$ & $(0.434)$ & $(0.500)$ & $(0.252)$ & $(0.639)$ & $(0.249)$ & $(0.410)$ & $(0.133)$ & (0.133) & $(0.633)$ \\
\hline Other races or more than one & $0.131 *$ & $4.430 * * *$ & 1.141 & 1.648 & 0.788 & 0.542 & & 1.972 & $2.229^{*}$ & 0.923 & 1.049 & 0.303 \\
\hline \multirow{2}{*}{\multicolumn{13}{|c|}{$\begin{array}{l}\text { Family variables } \\
\text { Number of children under } 18 \text { (ref: } 2 \text { children) }\end{array}$}} \\
\hline & & & & & & & & & & & & \\
\hline 0 child & 0.903 & $2.439 * *$ & 1.590 & $2.616^{* * * *}$ & $4.336 * * *$ & 1.383 & $2.312^{* * *}$ & 1.157 & 1.098 & $2.751^{* * *}$ & $2.127^{* * *}$ & 1.102 \\
\hline & $(0.347)$ & $(1.003)$ & $(0.459)$ & $(0.829)$ & $(1.769)$ & $(0.591)$ & $(0.849)$ & $(0.407)$ & $(0.391)$ & $(1.173)$ & $(0.781)$ & $(0.428)$ \\
\hline 1 child & 0.774 & $\begin{array}{l}1.649 \\
0.580\end{array}$ & 0.803 & 0.754 & $2.241^{*}$ & $\begin{array}{l}1.707 \\
0.059\end{array}$ & $2.202^{* * *}$ & 0.856 & 0.745 & $2.873 * * *$ & 1.367 & 0.713 \\
\hline 3 or more children & $(0.713)$ & $\left(\begin{array}{ccc}0.586) \\
1.675\end{array}\right.$ & $(0.263)$ & $(0.256)$ & 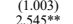 & $(0.559)$ & $(0.7355$ & $(0.266)$ & $(0.206)$ & $(1.0846)$ & $(0.4466)$ & $(0.240)$ \\
\hline 3 or more cinturent & $(0.276)$ & $(0.632)$ & $(0.146)$ & $(0.343)$ & $(0.952)$ & $(0.201)$ & $(0.406)$ & $\begin{array}{l}0.0206) \\
(0.206)\end{array}$ & $(0.211)$ & $\begin{array}{l}0.070 \\
(0.336)\end{array}$ & $\begin{array}{l}0.260 \\
(0.267)\end{array}$ & (0.172) \\
\hline \multirow{2}{*}{$\begin{array}{l}\text { Residence (ref: metro) } \\
\text { Not metro }\end{array}$} & 0.710 & 0.967 & 1.003 & 0.929 & 0.602 & 1.179 & 0.684 & 0.697 & 0.716 & 1.670 & 1.248 & 1.420 \\
\hline & $(0.248)$ & $(0.314)$ & $(0.298)$ & $(0.290)$ & $(0.214)$ & $(0.389)$ & $(0.209)$ & $(0.242)$ & $(0.214)$ & $(0.5)$ & $(0.418)$ & $(0.521)$ \\
\hline Not Identified & & 1.403 & 1.003 & $2.335 * *$ & 0.208 & 0.494 & 1.229 & 0.187 & 1.743 & $2.612^{*}$ & $0.174^{*}$ & 1.499 \\
\hline \multirow{2}{*}{ Unemployment (state) } & & $(0.853)$ & $(0.817)$ & $(0.917)$ & $(0.220)$ & $(0.335)$ & $(0.759)$ & $(0.195)$ & $(0.809)$ & $(1.355)$ & $(0.176)$ & $(0.878)$ \\
\hline & $\begin{array}{l}1.060 \\
(0.095)\end{array}$ & $\begin{array}{l}0.927 \\
(0.060)\end{array}$ & $\begin{array}{l}1.1 .151^{*} \\
(0.087)\end{array}$ & $\begin{array}{l}1.055 \\
(0.074)\end{array}$ & $\begin{array}{l}1.110 \\
(0.080)\end{array}$ & $\begin{array}{l}1.036 \\
(0.071)\end{array}$ & $\begin{array}{l}1.003 \\
(0.071)\end{array}$ & $\begin{array}{l}0.876^{* * *} \\
(0.052)\end{array}$ & $\begin{array}{l}0.883^{*} \\
(0.056)\end{array}$ & $\begin{array}{l}0.889 * * \\
(0.052)\end{array}$ & $\begin{array}{l}0.942 \\
(0.070)\end{array}$ & $\begin{array}{l}1.029 \\
(0.075)\end{array}$ \\
\hline \multirow{2}{*}{ Constant } & 29.818 & $0.000^{* * 4}$ & $0.000^{* * * *}$ & 0.160 & 0.075 & $0.000 * * * *$ & 0.023 & $\begin{array}{l}0.002^{* *} \\
0.04\end{array}$ & 1.918 & 0.002 & $0.004 *$ & $0.002^{* *}$ \\
\hline & $(88.819)$ & $(0.001)$ & $(0.000)$ & $(0.481)$ & $(0.237)$ & $(0.000)$ & $(0.075)$ & $(0.006)$ & $(5.531)$ & $(0.006)$ & $(0.014)$ & $(0.005)$ \\
\hline Observations & 2,464 & 2,546 & 2,528 & 2,533 & 2,480 & 2,464 & 2,325 & 2,368 & 2,334 & 2,298 & 2,347 & 2,353 \\
\hline
\end{tabular}

Notes: Standard errors in parentheses. ${ }^{* * *} \mathrm{p}<0.01,{ }^{* *} \mathrm{p}<0.05,{ }^{*} \mathrm{p}<0.1$

${ }^{(a)}$ Wife's age square is defined by age square divided by hundred. 
Table A4. Odds ratios for logit predicting wives' likelihood of labor force participation at $t$ as function of husband's labor force status in prior month, 2010 (wife in the labor force at $(\mathrm{t}-1))$.

\begin{tabular}{|c|c|c|c|c|c|c|c|c|}
\hline & $\begin{array}{c}\text { (1) } \\
\text { Jan: } \\
\text { wife in lf in } \\
(t-1)\end{array}$ & $\begin{array}{c}\text { (2) } \\
\text { Feb: } \\
\text { wife in lf } \\
\text { in }(t-1)\end{array}$ & $\begin{array}{c}\text { (3) } \\
\text { Mar: } \\
\text { wife in lf } \\
\text { in }(t-1)\end{array}$ & $\begin{array}{c}\text { (4) } \\
\text { Apr: } \\
\text { wife in lf } \\
\text { in }(t-1)\end{array}$ & $\begin{array}{c}(5) \\
\text { May: } \\
\text { wife in lf } \\
\text { in }(t-1)\end{array}$ & $\begin{array}{c}\text { (6) } \\
\text { Jun: } \\
\text { wife in lf } \\
\text { in }(t-1) \\
\end{array}$ & $\begin{array}{c}\text { (7) } \\
\text { Jul: } \\
\text { wife in lf } \\
\text { in }(t-1) \\
\end{array}$ & $\begin{array}{c}\text { (8) } \\
\text { Aug: } \\
\text { wife in lf } \\
\text { in (t-I) } \\
\end{array}$ \\
\hline \multicolumn{9}{|l|}{ Husband's characteristics } \\
\hline \multicolumn{9}{|l|}{ Husband's labor force status (ref: hus is emp at $t-1)$} \\
\hline Husband is unemployed at $(\mathrm{t}-1)$ & $\begin{array}{c}0.907 \\
(0.400) \\
0.586 \\
(0.260)\end{array}$ & $\begin{array}{c}0.600 \\
(0.317) \\
0.807 \\
(0.437)\end{array}$ & $\begin{array}{c}1.360 \\
(0.587) \\
1.530 \\
(0.927)\end{array}$ & $\begin{array}{c}0.661 \\
(0.383) \\
0.692 \\
(0.306)\end{array}$ & $\begin{array}{c}0.762 \\
(0.408) \\
0.961 \\
(0.405)\end{array}$ & $\begin{array}{c}0.546 \\
(0.255) \\
1.344 \\
(0.759)\end{array}$ & $\begin{array}{c}0.915 \\
(0.614) \\
0.553 \\
(0.207)\end{array}$ & $\begin{array}{c}0.965 \\
(0.799) \\
1.875 \\
(1.117)\end{array}$ \\
\hline $\begin{array}{l}\text { Husband's education (ref: some college) } \\
\text { Less than high school }\end{array}$ & $\begin{array}{c}0.543 \\
(0.236)\end{array}$ & $\begin{array}{c}1.006 \\
(0.473)\end{array}$ & $\begin{array}{c}0.978 \\
(0.477)\end{array}$ & $\begin{array}{c}1.333 \\
(0.627)\end{array}$ & $\begin{array}{c}1.226 \\
(0.640)\end{array}$ & $\begin{array}{c}0.617 \\
(0.267)\end{array}$ & $\begin{array}{c}0.674 \\
(0.274)\end{array}$ & $\begin{array}{c}0.791 \\
(0.373)\end{array}$ \\
\hline High school graduate & $\begin{array}{c}0.826 \\
(0.270)\end{array}$ & $\begin{array}{l}0.966 \\
(0.447)\end{array}$ & $\begin{array}{r}2.330^{* *} \\
(0.823)\end{array}$ & $\begin{array}{c}0.824 \\
(0.249)\end{array}$ & $\begin{array}{c}1.652 \\
(0.599)\end{array}$ & $\begin{array}{l}1.273 \\
(0.431)\end{array}$ & $\begin{array}{c}0.777 \\
(0.284)\end{array}$ & $\begin{array}{c}0.700 \\
(0.226)\end{array}$ \\
\hline Bachelor degree & $\begin{array}{c}0.876 \\
(0.338)\end{array}$ & $\begin{array}{c}0.668 \\
(0.227)\end{array}$ & $\begin{array}{c}1.932 \\
(0.774)\end{array}$ & $\begin{array}{c}0.977 \\
(0.340)\end{array}$ & $\begin{array}{r}2.444^{* *} \\
(1.098)\end{array}$ & $\begin{array}{c}1.008 \\
(0.391)\end{array}$ & $\begin{array}{c}0.642 \\
(0.216)\end{array}$ & $\begin{array}{c}1.923 \\
(0.873)\end{array}$ \\
\hline Masters or higher & $\begin{array}{c}0.873 \\
(0.476)\end{array}$ & $\begin{array}{l}0.603 \\
(0.398)\end{array}$ & $\begin{array}{c}0.812 \\
(0.353)\end{array}$ & $\begin{array}{c}0.561 \\
(0.235)\end{array}$ & $\begin{array}{l}1.285 \\
(0.704)\end{array}$ & $\begin{array}{c}0.885 \\
(0.582)\end{array}$ & $\begin{array}{l}0.479^{*} \\
(0.191)\end{array}$ & $\begin{array}{c}0.929 \\
(0.442)\end{array}$ \\
\hline Husband's age & $\begin{array}{l}1.020 \\
(0.026)\end{array}$ & $\begin{array}{l}0.988 \\
(0.033)\end{array}$ & $\begin{array}{l}1.055^{*} \\
(0.030)\end{array}$ & $\begin{array}{l}1.010 \\
(0.036)\end{array}$ & $\begin{array}{c}0.992 \\
(0.028)\end{array}$ & $\begin{array}{l}1.057^{*} \\
(0.032)\end{array}$ & $\begin{array}{c}0.981 \\
(0.033)\end{array}$ & $\begin{array}{c}0.997 \\
(0.030)\end{array}$ \\
\hline Husband's unemployment insurance (ref: no ins.) & $\begin{array}{c}0.670 \\
(0.347)\end{array}$ & $\begin{array}{l}0.908 \\
(0.583)\end{array}$ & $\begin{array}{c}0.455 \\
(0.216)\end{array}$ & & $\begin{array}{l}2.382 \\
(2.400)\end{array}$ & $\begin{array}{c}2.234 \\
(1.596)\end{array}$ & $\begin{array}{c}0.930 \\
(0.602)\end{array}$ & $\begin{array}{l}0.995 \\
(1.030)\end{array}$ \\
\hline \multicolumn{9}{|l|}{ Wife's characteristics } \\
\hline Less than high school & $\begin{array}{c}0.439^{* *} \\
(0.178)\end{array}$ & $\begin{array}{c}0.935 \\
(0.469)\end{array}$ & $\begin{array}{l}0.315^{* *} \\
(0.157)\end{array}$ & $\begin{array}{c}0.572 \\
(0.280)\end{array}$ & $\begin{array}{c}0.552 \\
(0.238)\end{array}$ & $\begin{array}{c}0.815 \\
(0.482)\end{array}$ & $\begin{array}{c}0.414^{* *} \\
(0.165)\end{array}$ & $\begin{array}{c}1.144 \\
(0.605)\end{array}$ \\
\hline High school graduate & $\begin{array}{c}1.213 \\
(0.416)\end{array}$ & $\begin{array}{c}1.364 \\
(0.592)\end{array}$ & $\begin{array}{c}0.465^{* *} \\
(0.147)\end{array}$ & $\begin{array}{c}0.851 \\
(0.294)\end{array}$ & $\begin{array}{c}1.383 \\
(0.457)\end{array}$ & $\begin{array}{c}0.471^{* *} \\
(0.143)\end{array}$ & $\begin{array}{c}0.930 \\
(0.330)\end{array}$ & $\begin{array}{c}1.020 \\
(0.345)\end{array}$ \\
\hline Bachelor degree & $\begin{array}{c}1.650 \\
(0.559)\end{array}$ & $\begin{array}{r}2.368^{* *} \\
(0.967)\end{array}$ & $\begin{array}{c}0.983 \\
(0.363)\end{array}$ & $\begin{array}{c}1.560 \\
(0.556)\end{array}$ & $\begin{array}{c}1.865 \\
(0.791)\end{array}$ & $\begin{array}{l}1.430 \\
(0.604)\end{array}$ & $\begin{array}{c}0.784 \\
(0.250)\end{array}$ & $\begin{array}{c}1.435 \\
(0.452)\end{array}$ \\
\hline Masters or higher & $\begin{array}{l}5.759 * * \\
(4.089)\end{array}$ & $\begin{array}{c}7.810^{* *} \\
(6.234)\end{array}$ & $\begin{array}{l}1.191 \\
(0.679)\end{array}$ & $\begin{array}{l}4.083^{* *} \\
(2.431)\end{array}$ & $\begin{array}{l}6.666^{* *} \\
(5.045)\end{array}$ & $\begin{array}{l}1.306 \\
(0.844)\end{array}$ & $\begin{array}{l}1.185 \\
(0.517)\end{array}$ & $\begin{array}{l}3.462^{*} \\
(2.182)\end{array}$ \\
\hline Wife's age & $\begin{array}{c}1.087 \\
(0.174)\end{array}$ & $\begin{array}{c}1.807 * * * \\
(0.367)\end{array}$ & $\begin{array}{c}1.080 \\
(0.151)\end{array}$ & $\begin{array}{c}0.859 \\
(0.175)\end{array}$ & $\begin{array}{c}1.163 \\
(0.169)\end{array}$ & $\begin{array}{c}0.812 \\
(0.133)\end{array}$ & $\begin{array}{c}0.897 \\
(0.172)\end{array}$ & $\begin{array}{c}0.942 \\
(0.165)\end{array}$ \\
\hline Wife's age square ${ }^{(a)}$ & $\begin{array}{c}0.903 \\
(0.178)\end{array}$ & $\begin{array}{c}0.493^{* * * *} \\
(0.124)\end{array}$ & $\begin{array}{c}0.877 \\
(0.156)\end{array}$ & $\begin{array}{l}1.268 \\
(0.331)\end{array}$ & $\begin{array}{c}0.861 \\
(0.162)\end{array}$ & $\begin{array}{c}1.344 \\
(0.290)\end{array}$ & $\begin{array}{c}1.214 \\
(0.279)\end{array}$ & $\begin{array}{l}1.088 \\
(0.237)\end{array}$ \\
\hline \multicolumn{9}{|l|}{ Wife's race \& ethnicity (ref: White alone) } \\
\hline Black & $\begin{array}{c}1.152 \\
(0.560)\end{array}$ & $\begin{array}{c}2.296 \\
(1.425)\end{array}$ & $\begin{array}{c}1.324 \\
(0.675)\end{array}$ & $\begin{array}{c}1.027 \\
(0.550)\end{array}$ & $\begin{array}{c}1.164 \\
(0.658)\end{array}$ & $\begin{array}{c}1.157 \\
(0.598)\end{array}$ & $\begin{array}{c}0.674 \\
(0.263)\end{array}$ & $\begin{array}{c}1.018 \\
(0.476)\end{array}$ \\
\hline Asian & $\begin{array}{c}0.878 \\
(0.423)\end{array}$ & $\begin{array}{c}0.794 \\
(0.465)\end{array}$ & $\begin{array}{c}0.352^{* * * *} \\
(0.111)\end{array}$ & $\begin{array}{c}1.701 \\
(1.237)\end{array}$ & $\begin{array}{c}0.898 \\
(0.483)\end{array}$ & $\begin{array}{c}1.087 \\
(0.683)\end{array}$ & $\begin{array}{c}0.696 \\
(0.285)\end{array}$ & $\begin{array}{l}1.002 \\
(0.606)\end{array}$ \\
\hline Other races or more than one & $\begin{array}{c}0.629 \\
(0.403)\end{array}$ & $\begin{array}{c}0.682 \\
(0.436)\end{array}$ & $\begin{array}{c}0.680 \\
(0.368)\end{array}$ & $\begin{array}{c}0.513 \\
(0.249)\end{array}$ & $\begin{array}{c}0.681 \\
(0.374)\end{array}$ & $\begin{array}{c}0.901 \\
(0.597)\end{array}$ & $\begin{array}{c}2.960 \\
(3.082)\end{array}$ & $\begin{array}{l}0.539^{*} \\
(0.185)\end{array}$ \\
\hline \multicolumn{9}{|l|}{ Family variables } \\
\hline 0 child & $\begin{array}{c}1.540 \\
(0.545)\end{array}$ & $\begin{array}{c}1.223 \\
(0.419)\end{array}$ & $\begin{array}{c}0.806 \\
(0.303)\end{array}$ & $\begin{array}{c}1.617 \\
(0.545)\end{array}$ & $\begin{array}{c}0.813 \\
(0.251)\end{array}$ & $\begin{array}{c}1.499 \\
(0.579)\end{array}$ & $\begin{array}{c}0.835 \\
(0.253)\end{array}$ & $\begin{array}{c}1.482 \\
(0.521)\end{array}$ \\
\hline 1 child & $\begin{array}{c}1.581 \\
(0.538)\end{array}$ & $\begin{array}{c}1.305 \\
(0.512)\end{array}$ & $\begin{array}{c}0.570 \\
(0.209)\end{array}$ & $\begin{array}{c}0.758 \\
(0.223)\end{array}$ & $\begin{array}{c}0.751 \\
(0.280)\end{array}$ & $\begin{array}{c}1.124 \\
(0.395)\end{array}$ & $\begin{array}{c}1.029 \\
(0.334)\end{array}$ & $\begin{array}{c}0.983 \\
(0.329)\end{array}$ \\
\hline 3 or more children & $\begin{array}{c}1.542 \\
(0.571)\end{array}$ & $\begin{array}{c}0.608 \\
(0.236)\end{array}$ & $\begin{array}{c}0.576 \\
(0.238)\end{array}$ & $\begin{array}{c}0.746 \\
(0.246)\end{array}$ & $\begin{array}{c}0.752 \\
(0.312)\end{array}$ & $\begin{array}{c}0.720 \\
(0.246)\end{array}$ & $\begin{array}{c}0.778 \\
(0.247)\end{array}$ & $\begin{array}{l}0.556^{*} \\
(0.193)\end{array}$ \\
\hline \multicolumn{9}{|l|}{ Residence (ref: metro) } \\
\hline Not metro & $\begin{array}{l}0.619^{*} \\
(0.158)\end{array}$ & $\begin{array}{c}0.916 \\
(0.338)\end{array}$ & $\begin{array}{c}0.652 \\
(0.199)\end{array}$ & $\begin{array}{c}0.775 \\
(0.280)\end{array}$ & $\begin{array}{c}1.133 \\
(0.359)\end{array}$ & $\begin{array}{c}0.661 \\
(0.182)\end{array}$ & $\begin{array}{c}1.084 \\
(0.321)\end{array}$ & $\begin{array}{c}0.800 \\
(0.238)\end{array}$ \\
\hline Not Identified & $\begin{array}{l}1.928 \\
(1.449)\end{array}$ & $\begin{array}{c}1.922 \\
(1.924)\end{array}$ & $\begin{array}{c}0.597 \\
(0.295)\end{array}$ & $\begin{array}{l}5.568^{*} \\
(5.684)\end{array}$ & $\begin{array}{c}1.099 \\
(0.710)\end{array}$ & $\begin{array}{c}0.421 \\
(0.246)\end{array}$ & $\begin{array}{c}0.634 \\
(0.345)\end{array}$ & $\begin{array}{c}1.141 \\
(0.709)\end{array}$ \\
\hline Unemployment (state) & $\begin{array}{c}1.071 \\
(0.066)\end{array}$ & $\begin{array}{l}1.078 \\
(0.080)\end{array}$ & $\begin{array}{c}0.982 \\
(0.054)\end{array}$ & $\begin{array}{c}0.952 \\
(0.065)\end{array}$ & $\begin{array}{c}0.825^{* *} \\
(0.071)\end{array}$ & $\begin{array}{c}0.959 \\
(0.051)\end{array}$ & $\begin{array}{c}1.089 \\
(0.073)\end{array}$ & $\begin{array}{c}1.007 \\
(0.053)\end{array}$ \\
\hline Constant & $\begin{array}{c}2.952 \\
(8.700)\end{array}$ & $\begin{array}{c}0.001^{* *} \\
(0.002)\end{array}$ & $\begin{array}{c}7.377 \\
(21.360)\end{array}$ & $\begin{array}{c}798.858^{*} \\
(3,004.97 \\
9)\end{array}$ & $\begin{array}{c}12.020 \\
(35.087)\end{array}$ & $\begin{array}{c}469.701^{*} \\
(1,586.67 \\
6)\end{array}$ & $\begin{array}{c}454.309^{*} \\
(1,669.08 \\
4)\end{array}$ & $\begin{array}{l}204.796^{*} \\
(646.662)\end{array}$ \\
\hline Observations & 6,727 & 6,645 & 6,597 & 6,216 & 6,438 & 6,358 & 6,328 & 6,258 \\
\hline
\end{tabular}

Notes: Standard errors in parentheses. $* * * \mathrm{p}<0.01, * * \mathrm{p}<0.05, * \mathrm{p}<0.1$

${ }^{(a)}$ Wife's age square is defined by age square divided by hundred. 
Table A5. Odds ratios for logit predicting wives' likelihood of labor force participation at $t$ as function of husband's labor force status in prior month, 2010 (wife not in the labor force at (t-1)).

\begin{tabular}{|c|c|c|c|c|c|c|c|c|}
\hline & (1) & (2) & (3) & (4) & (5) & (6) & (7) & $(8)$ \\
\hline & $\begin{array}{c}\text { Jan: } \\
\text { wife not in } \\
\text { lf in }(t-1)\end{array}$ & $\begin{array}{c}\text { Feb: } \\
\text { wife not in } \\
\text { lf in }(t-1)\end{array}$ & $\begin{array}{l}\text { Mar: } \\
\text { wife not in } \\
\text { lf in }(t-1)\end{array}$ & $\begin{array}{c}\text { Apr: } \\
\text { wife not in } \\
\text { lf in }(t-1)\end{array}$ & $\begin{array}{l}\text { May: } \\
\text { wife not in } \\
\text { lf in }(t-1)\end{array}$ & $\begin{array}{c}\text { Jun: } \\
\text { wife not in } \\
\text { lf in }(t-1)\end{array}$ & $\begin{array}{c}\text { Jul: } \\
\text { wife not in } \\
\text { lf in }(t-1)\end{array}$ & $\begin{array}{c}\text { Aug: } \\
\text { wife not in } \\
\text { lf in }(t-1)\end{array}$ \\
\hline \multicolumn{9}{|l|}{ Husband's characteristics } \\
\hline \multicolumn{9}{|l|}{ Husband's labor force status (ref: hus is emp at $t-I$ ) } \\
\hline Husband is unemployed at $(\mathrm{t}-1)$ & $\begin{array}{l}2.531^{*} \\
(1.265)\end{array}$ & $\begin{array}{c}2.238 \\
(1.247)\end{array}$ & $\begin{array}{l}1.462 \\
(0.613)\end{array}$ & $\begin{array}{c}0.774 \\
(0.819)\end{array}$ & $\begin{array}{c}0.520 \\
(0.590)\end{array}$ & $\begin{array}{c}1.991 \\
(1.212)\end{array}$ & $\begin{array}{c}0.474 \\
(0.380)\end{array}$ & $\begin{array}{c}1.103 \\
(0.605)\end{array}$ \\
\hline Husband not in labor force at (t-1) & $\begin{array}{l}1.156 \\
(0.665)\end{array}$ & $\begin{array}{c}1.239 \\
(0.566)\end{array}$ & $\begin{array}{c}0.936 \\
(0.449)\end{array}$ & $\begin{array}{l}2.535^{* *} \\
(1.115)\end{array}$ & $\begin{array}{c}1.507 \\
(0.688)\end{array}$ & $\begin{array}{l}1.341 \\
(0.608)\end{array}$ & $\begin{array}{c}1.353 \\
(0.666)\end{array}$ & $\begin{array}{l}2.087^{* *} \\
(0.749)\end{array}$ \\
\hline \multicolumn{9}{|l|}{ Husband's education (ref: some college) } \\
\hline Less than high school & $\begin{array}{c}0.505 \\
(0.267)\end{array}$ & $\begin{array}{c}1.011 \\
(0.459)\end{array}$ & $\begin{array}{c}2.510 * * * \\
(0.879)\end{array}$ & $\begin{array}{c}0.470 \\
(0.246)\end{array}$ & $\begin{array}{c}1.244 \\
(0.562)\end{array}$ & $\begin{array}{c}0.765 \\
(0.400)\end{array}$ & $\begin{array}{c}1.519 \\
(0.671)\end{array}$ & $\begin{array}{c}1.952 \\
(0.901)\end{array}$ \\
\hline High school graduate & $\begin{array}{l}1.129 \\
(0.374)\end{array}$ & $\begin{array}{c}0.840 \\
(0.308)\end{array}$ & $\begin{array}{c}2.295^{* * *} \\
(0.637)\end{array}$ & $\begin{array}{l}1.207 \\
(0.494)\end{array}$ & $\begin{array}{l}1.618 \\
(0.551)\end{array}$ & $\begin{array}{c}0.779 \\
(0.283)\end{array}$ & $\begin{array}{l}1.256 \\
(0.434)\end{array}$ & $\begin{array}{l}1.875^{*} \\
(0.612)\end{array}$ \\
\hline Bachelor degree & $\begin{array}{l}0.445^{*} \\
(0.204)\end{array}$ & $\begin{array}{c}1.081 \\
(0.361)\end{array}$ & $\begin{array}{c}1.787 \\
(0.632)\end{array}$ & $\begin{array}{c}0.259^{* *} \\
(0.147)\end{array}$ & $\begin{array}{c}1.084 \\
(0.567)\end{array}$ & $\begin{array}{c}0.632 \\
(0.280)\end{array}$ & $\begin{array}{c}0.540 \\
(0.265)\end{array}$ & $\begin{array}{c}0.999 \\
(0.380)\end{array}$ \\
\hline Masters or higher & $\begin{array}{c}0.274^{* *} \\
(0.171)\end{array}$ & $\begin{array}{c}1.273 \\
(0.643)\end{array}$ & $\begin{array}{l}1.068 \\
(0.552)\end{array}$ & $\begin{array}{c}0.292 \\
(0.220)\end{array}$ & $\begin{array}{c}0.674 \\
(0.375)\end{array}$ & $\begin{array}{c}0.480 \\
(0.249)\end{array}$ & $\begin{array}{c}0.584 \\
(0.313)\end{array}$ & $\begin{array}{c}0.890 \\
(0.353)\end{array}$ \\
\hline Husband's age & $\begin{array}{l}1.020 \\
(0.029)\end{array}$ & $\begin{array}{c}0.962 \\
(0.024)\end{array}$ & $\begin{array}{l}1.002 \\
(0.030)\end{array}$ & $\begin{array}{c}0.976 \\
(0.032)\end{array}$ & $\begin{array}{c}0.973 \\
(0.023)\end{array}$ & $\begin{array}{l}1.057^{*} \\
(0.030)\end{array}$ & $\begin{array}{c}0.972 \\
(0.027)\end{array}$ & $\begin{array}{l}1.043^{*} \\
(0.026)\end{array}$ \\
\hline Husband's unemployment insurance (ref: no ins.) & $\begin{array}{c}0.687 \\
(0.410)\end{array}$ & $\begin{array}{c}0.688 \\
(0.558)\end{array}$ & $\begin{array}{c}0.906 \\
(0.494)\end{array}$ & $\begin{array}{c}0.674 \\
(0.686)\end{array}$ & $\begin{array}{c}3.152 \\
(3.271)\end{array}$ & $\begin{array}{c}1.282 \\
(1.047)\end{array}$ & $\begin{array}{c}0.371 \\
(0.227)\end{array}$ & $\begin{array}{c}0.595 \\
(0.607)\end{array}$ \\
\hline \multicolumn{9}{|l|}{ Wife's characteristics } \\
\hline Less than high school & $\begin{array}{c}0.745 \\
(0.409)\end{array}$ & $\begin{array}{c}0.501 \\
(0.287)\end{array}$ & $\begin{array}{c}0.369 * * * \\
(0.137)\end{array}$ & $\begin{array}{c}0.666 \\
(0.277)\end{array}$ & $\begin{array}{c}0.707 \\
(0.318)\end{array}$ & $\begin{array}{c}0.714 \\
(0.403)\end{array}$ & $\begin{array}{c}0.339 * * \\
(0.163)\end{array}$ & $\begin{array}{c}0.530 \\
(0.250)\end{array}$ \\
\hline High school graduate & $\begin{array}{c}0.872 \\
(0.311)\end{array}$ & $\begin{array}{c}0.684 \\
(0.218)\end{array}$ & $\begin{array}{l}0.645^{*} \\
(0.170)\end{array}$ & $\begin{array}{c}0.860 \\
(0.306)\end{array}$ & $\begin{array}{c}0.699 \\
(0.221)\end{array}$ & $\begin{array}{c}0.772 \\
(0.225)\end{array}$ & $\begin{array}{c}0.733 \\
(0.248)\end{array}$ & $\begin{array}{c}0.463^{* * *} \\
(0.158)\end{array}$ \\
\hline Bachelor degree & $\begin{array}{l}1.720 \\
(0.735)\end{array}$ & $\begin{array}{c}0.538 \\
(0.237)\end{array}$ & $\begin{array}{c}0.371 * * \\
(0.145)\end{array}$ & $\begin{array}{c}0.767 \\
(0.369)\end{array}$ & $\begin{array}{c}1.299 \\
(0.582)\end{array}$ & $\begin{array}{c}1.158 \\
(0.486)\end{array}$ & $\begin{array}{c}0.838 \\
(0.369)\end{array}$ & $\begin{array}{c}2.087 * * \\
(0.698)\end{array}$ \\
\hline Masters or higher & $\begin{array}{c}2.200 \\
(1.374)\end{array}$ & $\begin{array}{c}0.629 \\
(0.348)\end{array}$ & $\begin{array}{c}0.961 \\
(0.487)\end{array}$ & $\begin{array}{c}1.884 \\
(1.193)\end{array}$ & $\begin{array}{c}1.718 \\
(0.995)\end{array}$ & $\begin{array}{c}0.789 \\
(0.649)\end{array}$ & $\begin{array}{l}1.410 \\
(0.749)\end{array}$ & $\begin{array}{c}1.817 \\
(0.743)\end{array}$ \\
\hline Wife's age & $\begin{array}{l}1.036 \\
(0.162)\end{array}$ & $\begin{array}{l}1.020 \\
(0.177)\end{array}$ & $\begin{array}{c}1.305 \\
(0.228)\end{array}$ & $\begin{array}{l}1.435^{*} \\
(0.287)\end{array}$ & $\begin{array}{l}1.078 \\
(0.213)\end{array}$ & $\begin{array}{c}1.053 \\
(0.182)\end{array}$ & $\begin{array}{c}0.889 \\
(0.161)\end{array}$ & $\begin{array}{c}0.877 \\
(0.140)\end{array}$ \\
\hline Wife's age square ${ }^{(a)}$ & $\begin{array}{c}0.933 \\
(0.195)\end{array}$ & $\begin{array}{c}1.004 \\
(0.219)\end{array}$ & $\begin{array}{l}0.673 * \\
(0.151)\end{array}$ & $\begin{array}{l}0.620^{*} \\
(0.156)\end{array}$ & $\begin{array}{c}0.907 \\
(0.226)\end{array}$ & $\begin{array}{c}0.825 \\
(0.181)\end{array}$ & $\begin{array}{l}1.117 \\
(0.257)\end{array}$ & $\begin{array}{c}1.098 \\
(0.219)\end{array}$ \\
\hline \multicolumn{9}{|l|}{ Wife's race \& ethnicity (ref: White alone) } \\
\hline Black & $\begin{array}{l}1.228 \\
(0.587)\end{array}$ & $\begin{array}{c}1.414 \\
(0.704)\end{array}$ & $\begin{array}{c}0.481 \\
(0.292)\end{array}$ & $\begin{array}{c}1.285 \\
(0.692)\end{array}$ & $\begin{array}{c}3.171^{* *} \\
(1.568)\end{array}$ & $\begin{array}{c}0.655 \\
(0.399)\end{array}$ & $\begin{array}{c}1.173 \\
(0.526)\end{array}$ & $\begin{array}{l}2.245^{*} \\
(0.921)\end{array}$ \\
\hline Asian & $\begin{array}{c}0.655 \\
(0.436)\end{array}$ & $\begin{array}{c}0.621 \\
(0.351)\end{array}$ & $\begin{array}{c}0.551 \\
(0.381)\end{array}$ & $\begin{array}{c}1.844 \\
(0.872)\end{array}$ & $\begin{array}{c}1.668 \\
(0.832)\end{array}$ & $\begin{array}{c}0.319 \\
(0.241)\end{array}$ & $\begin{array}{c}0.654 \\
(0.348)\end{array}$ & $\begin{array}{c}0.574 \\
(0.273)\end{array}$ \\
\hline Other races or more than one & $\begin{array}{c}1.062 \\
(0.788)\end{array}$ & $\begin{array}{c}0.734 \\
(0.619)\end{array}$ & $\begin{array}{c}0.694 \\
(0.518)\end{array}$ & $\begin{array}{l}1.505 \\
(1.196)\end{array}$ & $\begin{array}{c}0.404 \\
(0.307)\end{array}$ & $\begin{array}{l}1.518 \\
(0.795)\end{array}$ & $\begin{array}{c}0.837 \\
(0.522)\end{array}$ & $\begin{array}{l}1.325 \\
(0.699)\end{array}$ \\
\hline \multicolumn{9}{|l|}{ Family variables } \\
\hline 0 child & $\begin{array}{c}1.136 \\
(0.485)\end{array}$ & $\begin{array}{c}2.524^{* *} \\
(0.982)\end{array}$ & $\begin{array}{c}2.352 * * * \\
(0.720)\end{array}$ & $\begin{array}{c}2.206 \\
(1.078)\end{array}$ & $\begin{array}{c}3.193^{* *} \\
(1.470)\end{array}$ & $\begin{array}{l}2.262 * \\
(1.039)\end{array}$ & $\begin{array}{c}0.817 \\
(0.325)\end{array}$ & $\begin{array}{c}1.315 \\
(0.483)\end{array}$ \\
\hline 1 child & $\begin{array}{c}1.381 \\
(0.522)\end{array}$ & $\begin{array}{l}2.370^{* *} \\
(1.007)\end{array}$ & $\begin{array}{c}1.482 \\
(0.452)\end{array}$ & $\begin{array}{l}1.857 \\
(0.815)\end{array}$ & $\begin{array}{l}1.388 \\
(0.607)\end{array}$ & $\begin{array}{l}1.216 \\
(0.465)\end{array}$ & $\begin{array}{c}0.863 \\
(0.263)\end{array}$ & $\begin{array}{c}2.245^{* * *} * \\
(0.655)\end{array}$ \\
\hline 3 or more children & $\begin{array}{c}1.094 \\
(0.439)\end{array}$ & $\begin{array}{c}1.051 \\
(0.479)\end{array}$ & $\begin{array}{l}1.266 \\
(0.348)\end{array}$ & $\begin{array}{c}1.632 \\
(0.648)\end{array}$ & $\begin{array}{c}1.736 \\
(0.665)\end{array}$ & $\begin{array}{l}1.167 \\
(0.406)\end{array}$ & $\begin{array}{l}0.451^{*} \\
(0.182)\end{array}$ & $\begin{array}{c}1.347 \\
(0.385)\end{array}$ \\
\hline \multicolumn{9}{|l|}{ Residence (ref: metro) } \\
\hline Not metro & $\begin{array}{l}1.160 \\
(0.401)\end{array}$ & $\begin{array}{c}1.433 \\
(0.476)\end{array}$ & $\begin{array}{c}0.911 \\
(0.268)\end{array}$ & $\begin{array}{c}1.089 \\
(0.422)\end{array}$ & $\begin{array}{c}2.408^{* * *} \\
(0.774)\end{array}$ & $\begin{array}{c}2.452 * * * \\
(0.681)\end{array}$ & $\begin{array}{c}0.687 \\
(0.254)\end{array}$ & $\begin{array}{c}0.661 \\
(0.211)\end{array}$ \\
\hline Not Identified & $\begin{array}{c}0.742 \\
(0.548)\end{array}$ & $\begin{array}{c}1.274 \\
(1.041)\end{array}$ & $\begin{array}{l}1.052 \\
(0.613)\end{array}$ & $\begin{array}{c}1.681 \\
(1.050)\end{array}$ & $\begin{array}{c}1.360 \\
(1.069)\end{array}$ & $\begin{array}{c}1.938 \\
(0.977)\end{array}$ & $\begin{array}{c}1.318 \\
(0.819)\end{array}$ & $\begin{array}{c}0.851 \\
(0.584)\end{array}$ \\
\hline Unemployment (state) & $\begin{array}{c}0.937 \\
(0.072)\end{array}$ & $\begin{array}{c}0.893 \\
(0.069)\end{array}$ & $\begin{array}{c}0.960 \\
(0.058)\end{array}$ & $\begin{array}{c}1.110 \\
(0.093)\end{array}$ & $\begin{array}{c}1.005 \\
(0.072)\end{array}$ & $\begin{array}{c}0.926 \\
(0.066)\end{array}$ & $\begin{array}{c}0.927 \\
(0.068)\end{array}$ & $\begin{array}{c}1.009 \\
(0.083)\end{array}$ \\
\hline Constant & $\begin{array}{c}0.020 \\
(0.059)\end{array}$ & $\begin{array}{c}0.130 \\
(0.433)\end{array}$ & $\begin{array}{c}0.001^{* *} \\
(0.002)\end{array}$ & $\begin{array}{c}0.000^{* * * *} \\
(0.000)\end{array}$ & $\begin{array}{c}0.008 \\
(0.032)\end{array}$ & $\begin{array}{c}0.015 \\
(0.049)\end{array}$ & $\begin{array}{c}7.121 \\
(24.202)\end{array}$ & $\begin{array}{c}0.155 \\
(0.471)\end{array}$ \\
\hline Observations & 2,358 & 2,365 & 2,347 & 2,304 & 2,291 & 2,295 & 2,302 & 2,299 \\
\hline
\end{tabular}

Notes: Standard errors in parentheses. $* * * \mathrm{p}<0.01,{ }^{*} \mathrm{p}<0.05,{ }^{*} \mathrm{p}<0.1$

${ }^{(a)}$ Wife's age square is defined by age square divided by hundred. 
Table A6. Odds ratios predicting wives' likelihood of labor force participation at $t$ as function of husband's labor force status in prior month(s), 2008.

\begin{tabular}{|c|c|c|c|c|c|}
\hline & (1) & $(2)$ & (3) & $(4)$ & $(5)$ \\
\hline & $\begin{array}{c}\text { wife in lf } \\
\text { in }(t-1)\end{array}$ & $\begin{array}{l}\text { wife not in } \\
\text { lf in }(t-1)\end{array}$ & $\begin{array}{c}\text { wife in lf or } \\
\text { nlf in }(t-1)\end{array}$ & $\begin{array}{c}\text { wife in lf } \\
\text { in }(t-1)\end{array}$ & $\begin{array}{l}\text { wife not in } \\
\text { If in }(t-1)\end{array}$ \\
\hline \multicolumn{6}{|l|}{ Husband's characteristics } \\
\hline \multicolumn{6}{|l|}{ Husband's labor force status (ref: hus is emp at $t-1$ ) } \\
\hline \multirow[t]{2}{*}{ Husband is unemployed at $(\mathrm{t}-1)$} & 0.891 & 0.968 & 1.085 & 0.905 & 1.190 \\
\hline & $(0.222)$ & $(0.212)$ & $(0.110)$ & $(0.406)$ & $(0.300)$ \\
\hline \multirow[t]{2}{*}{ Husband not in labor force at (t-1) } & 0.953 & 1.077 & $0.843 * *$ & 0.924 & 1.290 \\
\hline & $(0.250)$ & $(0.173)$ & $(0.067)$ & $(0.255)$ & $(0.222)$ \\
\hline \multirow[t]{2}{*}{ Husband unemployed both in (t-1) \& (t-2) } & & & & 1.051 & 0.674 \\
\hline & & & & $(0.523)$ & $(0.218)$ \\
\hline \multicolumn{6}{|l|}{ Husband's education (ref: some college) } \\
\hline \multirow[t]{2}{*}{ Less than high school } & 0.812 & 0.841 & $0.758 * * *$ & 0.765 & 0.844 \\
\hline & $(0.186)$ & $(0.140)$ & $(0.075)$ & $(0.181)$ & $(0.163)$ \\
\hline \multirow[t]{2}{*}{ High school graduate } & 0.827 & 1.163 & 1.105 & 0.823 & 1.155 \\
\hline & $(0.103)$ & $(0.163)$ & $(0.075)$ & $(0.111)$ & $(0.173)$ \\
\hline \multirow[t]{2}{*}{ Bachelor degree } & 1.261 & $0.724 * *$ & $0.764 * * *$ & $1.327^{*}$ & $0.756^{*}$ \\
\hline & $(0.193)$ & $(0.109)$ & $(0.051)$ & $(0.217)$ & $(0.117)$ \\
\hline \multirow[t]{2}{*}{ Masters or higher } & 1.350 & $0.565 * * *$ & $0.431 * * *$ & $1.595^{*}$ & $0.608 * * *$ \\
\hline & $(0.383)$ & $(0.088)$ & $(0.034)$ & $(0.446)$ & $(0.098)$ \\
\hline \multirow[t]{2}{*}{ Husband's age } & 0.987 & 1.008 & 1.001 & 0.991 & 1.011 \\
\hline & $(0.015)$ & $(0.009)$ & $(0.005)$ & $(0.016)$ & $(0.009)$ \\
\hline \multirow[t]{2}{*}{ Husband's unemployment insurance (ref: no ins.) } & 1.043 & 1.245 & 1.105 & 1.111 & 1.302 \\
\hline & $(0.413)$ & $(0.465)$ & $(0.156)$ & $(0.467)$ & $(0.497)$ \\
\hline \multicolumn{6}{|l|}{ Wife's characteristics } \\
\hline \multicolumn{6}{|l|}{ Wife's education (ref: some college) } \\
\hline \multirow[t]{2}{*}{ Less than high school } & 0.796 & $0.734^{*}$ & $0.313 * * *$ & 0.784 & 0.804 \\
\hline & $(0.158)$ & $(0.127)$ & $(0.029)$ & $(0.171)$ & $(0.151)$ \\
\hline High school graduate & 0.862 & $0.838^{*}$ & $0.545 * * *$ & 0.798 & 0.881 \\
\hline & $(0.129)$ & $(0.088)$ & $(0.032)$ & $(0.123)$ & $(0.094)$ \\
\hline Bachelor degree & 0.913 & $1.471 * * *$ & $1.220 * * *$ & 0.858 & $1.411 * *$ \\
\hline & $(0.129)$ & $(0.180)$ & $(0.083)$ & $(0.129)$ & $(0.194)$ \\
\hline Masters or higher & 1.117 & $1.972 * * *$ & $2.334 * * *$ & 1.011 & $1.849 * * *$ \\
\hline & $(0.234)$ & $(0.335)$ & $(0.222)$ & $(0.211)$ & $(0.343)$ \\
\hline Wife's age & $1.285 * * *$ & $1.115^{*}$ & $1.161 * * *$ & $1.269 * * *$ & $1.126^{*}$ \\
\hline & $(0.101)$ & $(0.070)$ & $(0.043)$ & $(0.100)$ & $(0.077)$ \\
\hline Wife's age square ${ }^{(a)}$ & $0.759 * * *$ & $0.840 * *$ & $0.836 * * *$ & $0.766 * * *$ & $0.825 * *$ \\
\hline & $(0.076)$ & $(0.069)$ & $(0.038)$ & $(0.077)$ & $(0.073)$ \\
\hline Wife's race \& ethnicity (ref: White alone) & & & & & \\
\hline Black & $0.674 * *$ & $1.494 * * *$ & $1.448 * * *$ & $0.695^{*}$ & $1.503 * *$ \\
\hline & $(0.133)$ & $(0.196)$ & $(0.142)$ & $(0.138)$ & $(0.250)$ \\
\hline Asian & $0.590 * * *$ & $0.740 *$ & $0.674 * * *$ & $0.562 * * *$ & 0.836 \\
\hline & $(0.114)$ & $(0.124)$ & $(0.063)$ & $(0.110)$ & $(0.143)$ \\
\hline Other races or more than one & $0.543 * *$ & 0.939 & 1.020 & $0.589 * *$ & 0.868 \\
\hline & $(0.129)$ & $(0.263)$ & $(0.133)$ & $(0.154)$ & $(0.275)$ \\
\hline Family variables & & & & & \\
\hline Number of children under 18 (ref: 2 children) & & & & & \\
\hline 0 child & 1.133 & $1.270^{*}$ & $1.704 * * *$ & 1.030 & $1.288^{*}$ \\
\hline & $(0.167)$ & $(0.156)$ & $(0.117)$ & $(0.164)$ & $(0.166)$ \\
\hline 1 child & 0.927 & 1.071 & $1.303 * * *$ & 0.850 & 1.019 \\
\hline & $(0.118)$ & $(0.136)$ & $(0.075)$ & $(0.107)$ & $(0.136)$ \\
\hline 3 or more children & 0.803 & 0.848 & $0.608 * * *$ & $0.732 *$ & 0.886 \\
\hline & $(0.124)$ & $(0.094)$ & $(0.040)$ & $(0.124)$ & $(0.105)$ \\
\hline Residence (ref: metro) & & & & & \\
\hline Not metro & 1.138 & 1.010 & $1.186 * *$ & 1.268 & 1.003 \\
\hline & $(0.154)$ & $(0.127)$ & $(0.087)$ & $(0.183)$ & $(0.126)$ \\
\hline Not Identified & 1.235 & 1.236 & $1.313 * *$ & 1.228 & 1.029 \\
\hline & $(0.355)$ & $(0.254)$ & $(0.158)$ & $(0.374)$ & $(0.211)$ \\
\hline Unemployment (state) & $0.872 * * *$ & 1.016 & 0.977 & $0.896 * * *$ & 0.998 \\
\hline & $(0.035)$ & $(0.028)$ & $(0.015)$ & $(0.037)$ & $(0.031)$ \\
\hline Constant & 2.302 & $0.007 * * *$ & $0.182 * *$ & 2.194 & $0.006 * * *$ \\
\hline & (3.344) & $(0.008)$ & $(0.124)$ & $(3.203)$ & $(0.008)$ \\
\hline Observations & 44,668 & 15,821 & 60,489 & 36,220 & 12,609 \\
\hline
\end{tabular}

Notes: Standard errors in parentheses. ${ }^{* * *} \mathrm{p}<0.01,{ }^{* *} \mathrm{p}<0.05,{ }^{*} \mathrm{p}<0.1$

${ }^{(a)}$ Wife's age square is defined by age square divided by hundred. 
Table A7. Odds ratios for logit predicting wives' likelihood of labor force participation at $t$ as function of husband's labor force status in prior month(s), 2009.

\begin{tabular}{|c|c|c|c|c|c|}
\hline & (1) & $(2)$ & $(3)$ & $(4)$ & $(5)$ \\
\hline & $\begin{array}{c}\text { wife in lf } \\
\text { in }(t-1)\end{array}$ & $\begin{array}{l}\text { wife not in } \\
\text { lf in }(t-1)\end{array}$ & $\begin{array}{c}\text { wife in lf or } \\
\text { nlf in }(t-1)\end{array}$ & $\begin{array}{c}\text { wife in lf } \\
\text { in }(t-1)\end{array}$ & $\begin{array}{l}\text { wife not in } \\
\text { lf in }(t-1)\end{array}$ \\
\hline \multicolumn{6}{|l|}{ Husband's characteristics } \\
\hline \multicolumn{6}{|l|}{ Husband's labor force status (ref: hus is emp at $t-1$ ) } \\
\hline Husband is unemployed at $(\mathrm{t}-1)$ & $\begin{array}{c}0.762 * \\
(0.125)\end{array}$ & $\begin{array}{c}1.710 * * * \\
(0.261)\end{array}$ & $\begin{array}{l}1.182 * * \\
(0.090)\end{array}$ & $\begin{array}{c}0.674 * \\
(0.152)\end{array}$ & $\begin{array}{c}1.563 * * \\
(0.345)\end{array}$ \\
\hline Husband not in labor force at $(\mathrm{t}-1)$ & $\begin{array}{c}1.211 \\
(0.186)\end{array}$ & $\begin{array}{l}1.364 * * \\
(0.202)\end{array}$ & $\begin{array}{c}1.061 \\
(0.095)\end{array}$ & $\begin{array}{c}1.198 \\
(0.184)\end{array}$ & $\begin{array}{l}1.373 * * \\
(0.204)\end{array}$ \\
\hline Husband unemployed both in (t-1) \& (t-2) & & & & $\begin{array}{c}1.168 \\
(0.276)\end{array}$ & $\begin{array}{c}1.093 \\
(0.257)\end{array}$ \\
\hline \multicolumn{6}{|l|}{ Husband's education (ref: some college) } \\
\hline Less than high school & $\begin{array}{c}0.639 * * * \\
(0.085)\end{array}$ & $\begin{array}{c}0.942 \\
(0.130)\end{array}$ & $\begin{array}{c}0.728^{* * *} * \\
(0.060)\end{array}$ & $\begin{array}{c}0.639 * * * \\
(0.085)\end{array}$ & $\begin{array}{c}0.918 \\
(0.124)\end{array}$ \\
\hline High school graduate & $\begin{array}{c}0.921 \\
(0.099)\end{array}$ & $\begin{array}{c}0.940 \\
(0.094)\end{array}$ & $\begin{array}{c}1.070 \\
(0.068)\end{array}$ & $\begin{array}{c}0.934 \\
(0.100)\end{array}$ & $\begin{array}{c}0.945 \\
(0.094)\end{array}$ \\
\hline Bachelor degree & $\begin{array}{c}0.905 \\
(0.104)\end{array}$ & $\begin{array}{c}0.629 * * * \\
(0.072)\end{array}$ & $\begin{array}{c}0.720 * * * \\
(0.049)\end{array}$ & $\begin{array}{c}0.912 \\
(0.105)\end{array}$ & $\begin{array}{c}0.631 * * * \\
(0.072)\end{array}$ \\
\hline Masters or higher & $\begin{array}{c}0.587 * * * \\
(0.067)\end{array}$ & $\begin{array}{c}0.588 * * * \\
(0.079)\end{array}$ & $\begin{array}{c}0.428 * * * \\
(0.036)\end{array}$ & $\begin{array}{c}0.588 * * * \\
(0.067)\end{array}$ & $\begin{array}{c}0.591 * * * \\
(0.080)\end{array}$ \\
\hline Husband's age & $\begin{array}{c}1.004 \\
(0.008)\end{array}$ & $\begin{array}{c}0.997 \\
(0.008)\end{array}$ & $\begin{array}{c}1.003 \\
(0.004)\end{array}$ & $\begin{array}{c}1.004 \\
(0.008)\end{array}$ & $\begin{array}{c}0.998 \\
(0.009)\end{array}$ \\
\hline Husband's unemployment insurance (ref: no ins.) & $\begin{array}{c}1.203 \\
(0.252)\end{array}$ & $\begin{array}{c}0.855 \\
(0.166)\end{array}$ & $\begin{array}{c}1.069 \\
(0.106)\end{array}$ & $\begin{array}{c}1.189 \\
(0.250)\end{array}$ & $\begin{array}{c}0.870 \\
(0.173)\end{array}$ \\
\hline \multicolumn{6}{|l|}{ Wife's characteristics } \\
\hline \multicolumn{6}{|l|}{ Wife's education (ref: some college) } \\
\hline Less than high school & $\begin{array}{c}0.533 * * * \\
(0.067)\end{array}$ & $\begin{array}{l}0.758 * \\
(0.111)\end{array}$ & $\begin{array}{c}0.355^{* * *} * \\
(0.035)\end{array}$ & $\begin{array}{c}0.539 * * * \\
(0.068)\end{array}$ & $\begin{array}{l}0.767 * \\
(0.113)\end{array}$ \\
\hline High school graduate & $\begin{array}{c}0.751 * * * \\
(0.055)\end{array}$ & $\begin{array}{c}0.891 \\
(0.083)\end{array}$ & $\begin{array}{c}0.582 * * * \\
(0.033)\end{array}$ & $\begin{array}{c}0.754 * * * \\
(0.055)\end{array}$ & $\begin{array}{c}0.894 \\
(0.083)\end{array}$ \\
\hline Bachelor degree & $\begin{array}{c}1.313 * * * \\
(0.130)\end{array}$ & $\begin{array}{c}1.377 * * * \\
(0.163)\end{array}$ & $\begin{array}{c}1.400 * * * \\
(0.093)\end{array}$ & $\begin{array}{c}1.318^{* * *} \\
(0.130)\end{array}$ & $\begin{array}{c}1.376^{* * *} \\
(0.162)\end{array}$ \\
\hline Masters or higher & $\begin{array}{c}2.126^{* * * *} \\
(0.329)\end{array}$ & $\begin{array}{l}1.516 * * \\
(0.255)\end{array}$ & $\begin{array}{c}2.629 * * * \\
(0.281)\end{array}$ & $\begin{array}{c}2.126^{* * *} \\
(0.329)\end{array}$ & $\begin{array}{l}1.520 * * \\
(0.255)\end{array}$ \\
\hline Wife's age & $\begin{array}{c}1.168 * * * \\
(0.063)\end{array}$ & $\begin{array}{c}1.068 \\
(0.058)\end{array}$ & $\begin{array}{c}1.170 * * * \\
(0.041)\end{array}$ & $\begin{array}{c}1.175 * * * \\
(0.064)\end{array}$ & $\begin{array}{c}1.067 \\
(0.057)\end{array}$ \\
\hline Wife's age square ${ }^{(a)}$ & $\begin{array}{c}0.846 * * \\
(0.058)\end{array}$ & $\begin{array}{c}0.901 \\
(0.064)\end{array}$ & $\begin{array}{c}0.825 * * * \\
(0.037)\end{array}$ & $\begin{array}{l}0.839 * * \\
(0.058)\end{array}$ & $\begin{array}{c}0.901 \\
(0.064)\end{array}$ \\
\hline \multicolumn{6}{|l|}{ Wife's race \& ethnicity (ref: White alone) } \\
\hline Black & $\begin{array}{c}1.025 \\
(0.143)\end{array}$ & $\begin{array}{c}1.499 * * * \\
(0.222)\end{array}$ & $\begin{array}{c}1.445^{* * *} * \\
(0.139)\end{array}$ & $\begin{array}{c}1.018 \\
(0.143)\end{array}$ & $\begin{array}{c}1.482 * * * \\
(0.221)\end{array}$ \\
\hline Asian & $\begin{array}{c}0.905 \\
(0.149)\end{array}$ & $\begin{array}{c}0.690 * * \\
(0.116)\end{array}$ & $\begin{array}{c}0.621 * * * \\
(0.060)\end{array}$ & $\begin{array}{c}0.917 \\
(0.151)\end{array}$ & $\begin{array}{c}0.692 * * \\
(0.116)\end{array}$ \\
\hline Other races or more than one & $\begin{array}{c}0.859 \\
(0.146)\end{array}$ & $\begin{array}{c}1.165 \\
(0.243)\end{array}$ & $\begin{array}{c}1.023 \\
(0.137)\end{array}$ & $\begin{array}{c}0.851 \\
(0.145)\end{array}$ & $\begin{array}{c}1.172 \\
(0.245)\end{array}$ \\
\hline \multicolumn{6}{|l|}{ Family variables } \\
\hline \multicolumn{6}{|l|}{ Number of children under 18 (ref: 2 children) } \\
\hline 0 child & $\begin{array}{c}1.177 * \\
(0.104)\end{array}$ & $\begin{array}{c}1.703 * * * \\
(0.193)\end{array}$ & $\begin{array}{c}1.927 * * * \\
(0.132)\end{array}$ & $\begin{array}{c}1.168 * \\
(0.105)\end{array}$ & $\begin{array}{c}1.722 * * * \\
(0.196)\end{array}$ \\
\hline \multirow[b]{2}{*}{3 or more children } & $\begin{array}{c}0.954 \\
(0.084)\end{array}$ & $\begin{array}{c}1.188 \\
(0.126)\end{array}$ & $\begin{array}{c}1.205^{* * *} * \\
(0.073)\end{array}$ & $\begin{array}{c}0.946 \\
(0.084)\end{array}$ & $\begin{array}{c}1.184 \\
(0.127)\end{array}$ \\
\hline & $\begin{array}{l}0.834^{*} \\
(0.087)\end{array}$ & $\begin{array}{c}0.804 * * \\
(0.081)\end{array}$ & $\begin{array}{c}0.579 * * * \\
(0.037)\end{array}$ & $\begin{array}{l}0.826^{*} \\
(0.087)\end{array}$ & $\begin{array}{c}0.811 * * \\
(0.082)\end{array}$ \\
\hline Residence (ref: metro) & & & & & \\
\hline Not metro & $\begin{array}{c}1.087 \\
(0.101)\end{array}$ & $\begin{array}{c}0.931 \\
(0.100)\end{array}$ & $\begin{array}{c}1.103 \\
(0.084)\end{array}$ & $\begin{array}{c}1.096 \\
(0.103)\end{array}$ & $\begin{array}{c}0.933 \\
(0.101)\end{array}$ \\
\hline Not Identified & $\begin{array}{c}1.171 \\
(0.237)\end{array}$ & $\begin{array}{c}0.960 \\
(0.194)\end{array}$ & $\begin{array}{c}1.236 \\
(0.176)\end{array}$ & $\begin{array}{c}1.163 \\
(0.235)\end{array}$ & $\begin{array}{c}0.965 \\
(0.195)\end{array}$ \\
\hline Unemployment (state) & $\begin{array}{c}0.995 \\
(0.018)\end{array}$ & $\begin{array}{c}0.995 \\
(0.019)\end{array}$ & $\begin{array}{c}0.976 * * \\
(0.010)\end{array}$ & $\begin{array}{c}0.995 \\
(0.018)\end{array}$ & $\begin{array}{c}0.995 \\
(0.019)\end{array}$ \\
\hline Constant & $\begin{array}{c}2.490 \\
(2.469)\end{array}$ & $\begin{array}{c}0.017 * * * \\
(0.018)\end{array}$ & $\begin{array}{c}0.168 * * \\
(0.115)\end{array}$ & $\begin{array}{c}2.220 \\
(2.238)\end{array}$ & $\begin{array}{c}0.018 * * * \\
(0.018)\end{array}$ \\
\hline Observations & 87,457 & 29,282 & 116,739 & 86,877 & 29,083 \\
\hline
\end{tabular}

Notes: Standard errors in parentheses. ${ }^{* * *} \mathrm{p}<0.01,{ }^{* *} \mathrm{p}<0.05,{ }^{*} \mathrm{p}<0.1$

${ }^{(a)}$ Wife's age square is defined by age square divided by hundred. 
Table A8. Odds ratios predicting wives' likelihood of labor force participation at $t$ as function of husband's labor force status in prior month(s), 2010.

\begin{tabular}{|c|c|c|c|c|c|}
\hline & $(1)$ & $(2)$ & $(3)$ & (4) & $(5)$ \\
\hline & $\begin{array}{l}\text { wife in lf } \\
\text { in }(t-1)\end{array}$ & $\begin{array}{l}\text { wife not in } \\
\text { lf in }(t-1)\end{array}$ & $\begin{array}{c}\text { wife in lf or } \\
\text { nlf in }(t-1)\end{array}$ & $\begin{array}{l}\text { wife in lf } \\
\text { in }(t-1)\end{array}$ & $\begin{array}{l}\text { wife not in } \\
\text { If in }(t-1)\end{array}$ \\
\hline \multicolumn{6}{|l|}{ Husband's characteristics } \\
\hline \multicolumn{6}{|l|}{ Husband's labor force status (ref: hus is emp at $t-1$ ) } \\
\hline Husband is unemployed at $(\mathrm{t}-1)$ & $\begin{array}{c}0.827 \\
(0.148)\end{array}$ & $\begin{array}{c}1.332 \\
(0.243)\end{array}$ & $\begin{array}{c}1.277 * * * \\
(0.114)\end{array}$ & $\begin{array}{c}0.816 \\
(0.251)\end{array}$ & $\begin{array}{c}1.028 \\
(0.310)\end{array}$ \\
\hline Husband not in labor force at (t-1) & $\begin{array}{c}0.943 \\
(0.171)\end{array}$ & $\begin{array}{l}1.448 * * \\
(0.243)\end{array}$ & $\begin{array}{c}0.994 \\
(0.093)\end{array}$ & $\begin{array}{c}0.933 \\
(0.170)\end{array}$ & $\begin{array}{l}1.460 * * \\
(0.247)\end{array}$ \\
\hline Husband unemployed both in (t-1) \& (t-2) & & & & $\begin{array}{c}1.042 \\
(0.355)\end{array}$ & $\begin{array}{c}1.326 \\
(0.424)\end{array}$ \\
\hline \multicolumn{6}{|l|}{ Husband's education (ref: some college) } \\
\hline Less than high school & $\begin{array}{c}0.892 \\
(0.137)\end{array}$ & $\begin{array}{c}1.157 \\
(0.204)\end{array}$ & $\begin{array}{c}0.730 * * * \\
(0.066)\end{array}$ & $\begin{array}{c}0.888 \\
(0.137)\end{array}$ & $\begin{array}{c}1.170 \\
(0.208)\end{array}$ \\
\hline High school graduate & $\begin{array}{c}1.057 \\
(0.114)\end{array}$ & $\begin{array}{l}1.300 * * \\
(0.171)\end{array}$ & $\begin{array}{c}1.090 \\
(0.074)\end{array}$ & $\begin{array}{c}1.057 \\
(0.117)\end{array}$ & $\begin{array}{l}1.319 * * \\
(0.173)\end{array}$ \\
\hline Bachelor degree & $\begin{array}{l}1.290 * \\
(0.181)\end{array}$ & $\begin{array}{l}0.758^{*} \\
(0.123)\end{array}$ & $\begin{array}{c}0.754 * * * \\
(0.055)\end{array}$ & $\begin{array}{l}1.280 * \\
(0.179)\end{array}$ & $\begin{array}{l}0.762 * \\
(0.124)\end{array}$ \\
\hline Masters or higher & $\begin{array}{c}0.848 \\
(0.154)\end{array}$ & $\begin{array}{c}0.581 * * * \\
(0.116)\end{array}$ & $\begin{array}{c}0.440 * * * \\
(0.039)\end{array}$ & $\begin{array}{c}0.849 \\
(0.160)\end{array}$ & $\begin{array}{c}0.588 * * * \\
(0.118)\end{array}$ \\
\hline Husband's age & $\begin{array}{c}1.008 \\
(0.010)\end{array}$ & $\begin{array}{c}1.010 \\
(0.011)\end{array}$ & $\begin{array}{c}0.998 \\
(0.005)\end{array}$ & $\begin{array}{c}1.008 \\
(0.010)\end{array}$ & $\begin{array}{c}1.010 \\
(0.011)\end{array}$ \\
\hline Husband's unemployment insurance (ref: no ins.) & $\begin{array}{c}1.066 \\
(0.236)\end{array}$ & $\begin{array}{c}0.851 \\
(0.225)\end{array}$ & $\begin{array}{c}0.954 \\
(0.099)\end{array}$ & $\begin{array}{c}1.038 \\
(0.229)\end{array}$ & $\begin{array}{c}0.870 \\
(0.234)\end{array}$ \\
\hline \multicolumn{6}{|l|}{ Wife's characteristics } \\
\hline \multicolumn{6}{|l|}{ Wife's education (ref: some college) } \\
\hline Less than high school & $\begin{array}{c}0.595 * * * \\
(0.100)\end{array}$ & $\begin{array}{c}0.526 * * * \\
(0.096)\end{array}$ & $\begin{array}{c}0.377 * * * \\
(0.038)\end{array}$ & $\begin{array}{c}0.593 * * * \\
(0.100)\end{array}$ & $\begin{array}{c}0.526 * * * \\
(0.096)\end{array}$ \\
\hline High school graduate & $\begin{array}{c}0.921 \\
(0.103)\end{array}$ & $\begin{array}{l}0.721 * * \\
(0.094)\end{array}$ & $\begin{array}{c}0.617 * * * \\
(0.037)\end{array}$ & $\begin{array}{c}0.916 \\
(0.103)\end{array}$ & $\begin{array}{l}0.721 * * \\
(0.094)\end{array}$ \\
\hline Bachelor degree & $\begin{array}{c}1.388 * * * \\
(0.165)\end{array}$ & $\begin{array}{c}1.054 \\
(0.164)\end{array}$ & $\begin{array}{c}1.352 * * * \\
(0.092)\end{array}$ & $\begin{array}{c}1.398 * * * \\
(0.169)\end{array}$ & $\begin{array}{c}1.039 \\
(0.163)\end{array}$ \\
\hline Masters or higher & $\begin{array}{c}2.356 * * * \\
(0.497)\end{array}$ & $\begin{array}{l}1.415^{*} \\
(0.294)\end{array}$ & $\begin{array}{c}2.850 * * * \\
(0.332)\end{array}$ & $\begin{array}{c}2.376 * * * \\
(0.506)\end{array}$ & $\begin{array}{l}1.426^{*} \\
(0.299)\end{array}$ \\
\hline Wife's age & $\begin{array}{c}1.025 \\
(0.053)\end{array}$ & $\begin{array}{c}1.042 \\
(0.061)\end{array}$ & $\begin{array}{c}1.224 * * * \\
(0.046)\end{array}$ & $\begin{array}{c}1.021 \\
(0.054)\end{array}$ & $\begin{array}{c}1.048 \\
(0.061)\end{array}$ \\
\hline Wife's age square ${ }^{(a)}$ & $\begin{array}{c}0.996 \\
(0.065)\end{array}$ & $\begin{array}{c}0.903 \\
(0.066)\end{array}$ & $\begin{array}{c}0.784 * * * \\
(0.036)\end{array}$ & $\begin{array}{c}1.002 \\
(0.067)\end{array}$ & $\begin{array}{c}0.896 \\
(0.065)\end{array}$ \\
\hline \multicolumn{6}{|l|}{ Wife's race \& ethnicity (ref: White alone) } \\
\hline Black & $\begin{array}{c}1.012 \\
(0.174)\end{array}$ & $\begin{array}{l}1.415 * * \\
(0.238)\end{array}$ & $\begin{array}{c}1.359 * * * \\
(0.137)\end{array}$ & $\begin{array}{c}1.004 \\
(0.172)\end{array}$ & $\begin{array}{l}1.442 * * \\
(0.242)\end{array}$ \\
\hline Asian & $\begin{array}{c}0.771 \\
(0.125)\end{array}$ & $\begin{array}{c}0.836 \\
(0.171)\end{array}$ & $\begin{array}{c}0.592 * * * \\
(0.060)\end{array}$ & $\begin{array}{c}0.778 \\
(0.128)\end{array}$ & $\begin{array}{c}0.853 \\
(0.176)\end{array}$ \\
\hline Other races or more than one & $\begin{array}{c}0.726 \\
(0.146)\end{array}$ & $\begin{array}{c}1.158 \\
(0.254)\end{array}$ & $\begin{array}{c}0.878 \\
(0.117)\end{array}$ & $\begin{array}{c}0.757 \\
(0.156)\end{array}$ & $\begin{array}{c}1.170 \\
(0.259)\end{array}$ \\
\hline \multicolumn{6}{|l|}{ Family variables } \\
\hline 0 child & $\begin{array}{c}1.036 \\
(0.121)\end{array}$ & $\begin{array}{c}1.726 * * * \\
(0.259)\end{array}$ & $\begin{array}{c}1.872 * * * \\
(0.129)\end{array}$ & $\begin{array}{c}1.041 \\
(0.121)\end{array}$ & $\begin{array}{c}1.707 * * * \\
(0.259)\end{array}$ \\
\hline 1 child & $\begin{array}{c}0.961 \\
(0.108)\end{array}$ & $\begin{array}{c}1.434 * * * \\
(0.187)\end{array}$ & $\begin{array}{c}1.245 * * * \\
(0.076)\end{array}$ & $\begin{array}{c}0.961 \\
(0.108)\end{array}$ & $\begin{array}{c}1.433 * * * \\
(0.186)\end{array}$ \\
\hline 3 or more children & $\begin{array}{c}0.739 * * \\
(0.090)\end{array}$ & $\begin{array}{c}1.079 \\
(0.147)\end{array}$ & $\begin{array}{c}0.581 * * * \\
(0.042)\end{array}$ & $\begin{array}{c}0.742 * * \\
(0.090)\end{array}$ & $\begin{array}{c}1.074 \\
(0.146)\end{array}$ \\
\hline \multicolumn{6}{|l|}{ Residence (ref: metro) } \\
\hline Not metro & $\begin{array}{c}0.786 * * \\
(0.075)\end{array}$ & $\begin{array}{c}1.158 \\
(0.133)\end{array}$ & $\begin{array}{c}1.049 \\
(0.082)\end{array}$ & $\begin{array}{c}0.786 * * \\
(0.076)\end{array}$ & $\begin{array}{c}1.166 \\
(0.134)\end{array}$ \\
\hline Not Identified & $\begin{array}{c}0.903 \\
(0.196)\end{array}$ & $\begin{array}{c}1.220 \\
(0.265)\end{array}$ & $\begin{array}{c}1.165 \\
(0.175)\end{array}$ & $\begin{array}{c}0.896 \\
(0.195)\end{array}$ & $\begin{array}{c}1.222 \\
(0.264)\end{array}$ \\
\hline Unemployment (state) & $\begin{array}{c}0.983 \\
(0.022)\end{array}$ & $\begin{array}{c}0.959 \\
(0.024)\end{array}$ & $\begin{array}{c}0.953 * * * \\
(0.012)\end{array}$ & $\begin{array}{c}0.983 \\
(0.023)\end{array}$ & $\begin{array}{l}0.957^{*} \\
(0.024)\end{array}$ \\
\hline Constant & $\begin{array}{c}25.277^{* *} \\
* \\
(24.445)\end{array}$ & $0.030 * * *$ & $0.090 * * *$ & $\begin{array}{c}26.755^{* *} \\
* \\
(26.527)\end{array}$ & $0.028 * * *$ \\
\hline Observations & 52,299 & 18,690 & 70,989 & 51,966 & 18,574 \\
\hline
\end{tabular}

Notes: Standard errors in parentheses. ${ }^{* * *} \mathrm{p}<0.01,{ }^{* *} \mathrm{p}<0.05,{ }^{*} \mathrm{p}<0.1$

${ }^{(a)}$ Wife's age square is defined by age square divided by hundred. 
Table A9. Odds ratios from logit predicting wives' likelihood of labor force participation at $t$ as function of husband's unemployment in prior month, $(t-1): 2008,2009 \& 2010$.

\begin{tabular}{|c|c|c|c|c|}
\hline & $(1)$ & $(2)$ & (3) & (4) \\
\hline & \multicolumn{2}{|c|}{$\begin{array}{l}\text { month dummies \& } \\
\text { interaction terms }\end{array}$} & \multicolumn{2}{|c|}{$\begin{array}{l}\text { month dummies interaction } \\
\text { terms with controls }\end{array}$} \\
\hline & $\begin{array}{c}\text { wife in lf in } \\
(t-1)\end{array}$ & $\begin{array}{l}\text { wife not in } \\
\text { lf in }(t-1)\end{array}$ & $\begin{array}{c}\text { wife in lf in } \\
(t-1)\end{array}$ & $\begin{array}{c}\text { wife not in lf } \\
\text { in }(t-1)\end{array}$ \\
\hline \multicolumn{5}{|l|}{ Monthly dummies (ref:Dec2008) } \\
\hline Aug2008 & $\begin{array}{l}1.505^{* *} \\
(0.275)\end{array}$ & $\begin{array}{c}0.924 \\
(0.137)\end{array}$ & $\begin{array}{l}1.487 * * \\
(0.273)\end{array}$ & $\begin{array}{c}0.915 \\
(0.137)\end{array}$ \\
\hline Sep2008 & $\begin{array}{c}0.830 \\
(0.139)\end{array}$ & $\begin{array}{l}1.798 * * * \\
(0.217)\end{array}$ & $\begin{array}{c}0.822 \\
(0.139)\end{array}$ & $\begin{array}{l}1.801 * * * \\
(0.218)\end{array}$ \\
\hline Oct 2008 & $\begin{array}{c}0.838 \\
(0.125)\end{array}$ & $\begin{array}{l}1.480 * * * \\
(0.176)\end{array}$ & $\begin{array}{c}0.835 \\
(0.124)\end{array}$ & $\begin{array}{l}1.499 * * * \\
(0.178)\end{array}$ \\
\hline Nov2008 & $\begin{array}{l}0.739 * * \\
(0.109)\end{array}$ & $\begin{array}{c}1.069 \\
(0.141)\end{array}$ & $\begin{array}{l}0.742 * * \\
(0.110)\end{array}$ & $\begin{array}{l}1.089 \\
(0.144)\end{array}$ \\
\hline Jan2009 & $\begin{array}{l}0.547 * * * \\
(0.080)\end{array}$ & $\begin{array}{c}1.017 \\
(0.144)\end{array}$ & $\begin{array}{c}0.559 * * * \\
(0.083)\end{array}$ & $\begin{array}{l}1.046 \\
(0.150)\end{array}$ \\
\hline Feb2009 & $\begin{array}{l}0.742^{*} \\
(0.116)\end{array}$ & $\begin{array}{c}0.880 \\
(0.133)\end{array}$ & $\begin{array}{l}0.764^{*} \\
(0.123)\end{array}$ & $\begin{array}{c}0.912 \\
(0.142)\end{array}$ \\
\hline Mar2009 & $\begin{array}{c}0.672 * * \\
(0.103)\end{array}$ & $\begin{array}{l}0.779 * \\
(0.112)\end{array}$ & $\begin{array}{c}0.699 * * \\
(0.110)\end{array}$ & $\begin{array}{c}0.816 \\
(0.123)\end{array}$ \\
\hline Apr2009 & $\begin{array}{c}0.859 \\
(0.137)\end{array}$ & $\begin{array}{c}1.029 \\
(0.140)\end{array}$ & $\begin{array}{c}0.900 \\
(0.139)\end{array}$ & $\begin{array}{l}1.085 \\
(0.155)\end{array}$ \\
\hline May2009 & $\begin{array}{c}0.640 * * * \\
(0.097)\end{array}$ & $\begin{array}{c}0.881 \\
(0.126)\end{array}$ & $\begin{array}{c}0.678^{* *} \\
(0.106)\end{array}$ & $\begin{array}{c}0.940 \\
(0.140)\end{array}$ \\
\hline Jun2009 & $\begin{array}{c}0.794 \\
(0.112)\end{array}$ & $\begin{array}{c}0.959 \\
(0.132)\end{array}$ & $\begin{array}{c}0.843 \\
(0.125)\end{array}$ & $\begin{array}{l}1.027 \\
(0.143)\end{array}$ \\
\hline Jul2009 & $\begin{array}{c}0.606^{* * *} * \\
(0.089)\end{array}$ & $\begin{array}{c}1.062 \\
(0.147)\end{array}$ & $\begin{array}{c}0.643 * * * \\
(0.099)\end{array}$ & $\begin{array}{l}1.148 \\
(0.177)\end{array}$ \\
\hline Aug2009 & $\begin{array}{c}0.613 * * * \\
(0.086)\end{array}$ & $\begin{array}{c}1.187 \\
(0.162)\end{array}$ & $\begin{array}{c}0.655 * * * \\
(0.102)\end{array}$ & $\begin{array}{l}1.287^{*} \\
(0.189)\end{array}$ \\
\hline Sep2009 & $\begin{array}{c}0.708^{* *} \\
(0.118)\end{array}$ & $\begin{array}{l}1.305^{* *} \\
(0.157)\end{array}$ & $\begin{array}{c}0.755 \\
(0.133)\end{array}$ & $\begin{array}{c}1.415^{* * * *} \\
(0.179)\end{array}$ \\
\hline Oct2009 & $\begin{array}{c}0.655^{* *} \\
(0.109)\end{array}$ & $\begin{array}{c}0.801 \\
(0.139)\end{array}$ & $\begin{array}{c}0.702 * * \\
(0.119)\end{array}$ & $\begin{array}{c}0.864 \\
(0.163)\end{array}$ \\
\hline Nov2009 & $\begin{array}{c}0.619 * * * \\
(0.097)\end{array}$ & $\begin{array}{c}0.903 \\
(0.139)\end{array}$ & $\begin{array}{c}0.660 * * \\
(0.105)\end{array}$ & $\begin{array}{c}0.976 \\
(0.163)\end{array}$ \\
\hline Dec2009 & $\begin{array}{c}0.505 * * * \\
(0.067)\end{array}$ & $\begin{array}{l}0.769^{*} \\
(0.116)\end{array}$ & $\begin{array}{c}0.536^{* * *} \\
(0.077)\end{array}$ & $\begin{array}{c}0.831 \\
(0.139)\end{array}$ \\
\hline $\operatorname{Jan} 2010$ & $\begin{array}{c}0.606 * * * \\
(0.096)\end{array}$ & $\begin{array}{c}0.796 \\
(0.133)\end{array}$ & $\begin{array}{c}0.642 * * * \\
(0.108)\end{array}$ & $\begin{array}{c}0.858 \\
(0.155)\end{array}$ \\
\hline Feb2010 & $\begin{array}{c}0.793 \\
(0.127)\end{array}$ & $\begin{array}{c}0.715^{* *} \\
(0.110)\end{array}$ & $\begin{array}{c}0.835 \\
(0.146)\end{array}$ & $\begin{array}{c}0.767 \\
(0.126)\end{array}$ \\
\hline Mar2010 & $\begin{array}{c}0.640 * * * \\
(0.093)\end{array}$ & $\begin{array}{c}1.066 \\
(0.145)\end{array}$ & $\begin{array}{c}0.675^{* *} \\
(0.106)\end{array}$ & $\begin{array}{c}1.140 \\
(0.159)\end{array}$ \\
\hline Apr2010 & $\begin{array}{c}0.681 * * \\
(0.109)\end{array}$ & $\begin{array}{c}0.698^{* *} \\
(0.114)\end{array}$ & $\begin{array}{l}0.717 * \\
(0.120)\end{array}$ & $\begin{array}{l}0.746^{*} \\
(0.131)\end{array}$ \\
\hline May2010 & $\begin{array}{c}0.524 * * * \\
(0.082)\end{array}$ & $\begin{array}{c}0.809 \\
(0.122)\end{array}$ & $\begin{array}{c}0.549 * * * \\
(0.089)\end{array}$ & $\begin{array}{c}0.871 \\
(0.138)\end{array}$ \\
\hline Jun2010 & $\begin{array}{c}0.627 * * * \\
(0.103)\end{array}$ & $\begin{array}{c}0.875 \\
(0.114)\end{array}$ & $\begin{array}{c}0.657 * * \\
(0.114)\end{array}$ & $\begin{array}{c}0.933 \\
(0.128)\end{array}$ \\
\hline Jul2010 & $\begin{array}{c}0.574 * * * \\
(0.082)\end{array}$ & $\begin{array}{c}1.079 \\
(0.156)\end{array}$ & $\begin{array}{c}0.603 * * * \\
(0.089)\end{array}$ & $\begin{array}{c}1.153 \\
(0.178)\end{array}$ \\
\hline Aug2010 & $\begin{array}{c}0.677 * * * \\
(0.096)\end{array}$ & $\begin{array}{l}1.281 * \\
(0.175)\end{array}$ & $\begin{array}{c}0.714 * * \\
(0.107)\end{array}$ & $\begin{array}{l}1.367 * * \\
(0.188)\end{array}$ \\
\hline $\begin{array}{l}\text { Husband's characteristics } \\
\text { Interaction terms }\end{array}$ & & & & \\
\hline Husband is unemployed ${ }^{*}$ December 2008 (ref.) & $\begin{array}{c}1.222 \\
(0.759)\end{array}$ & $\begin{array}{l}1.898 * * \\
(0.592)\end{array}$ & $\begin{array}{l}1.392 \\
(0.871)\end{array}$ & $\begin{array}{l}1.880^{* *} \\
(0.599)\end{array}$ \\
\hline Husband is unemployed ${ }^{*}$ August 2008 & $\begin{array}{c}0.677 \\
(0.444)\end{array}$ & $\begin{array}{c}0.864 \\
(0.450)\end{array}$ & $\begin{array}{c}0.759 \\
(0.502)\end{array}$ & $\begin{array}{c}0.841 \\
(0.443)\end{array}$ \\
\hline Husband is unemployed ${ }^{*}$ September 2008 & $\begin{array}{l}0.485 \\
(0.222)\end{array}$ & $\begin{array}{l}0.979 \\
(0.464)\end{array}$ & $\begin{array}{c}0.544 \\
(0.250)\end{array}$ & $\begin{array}{c}0.959 \\
(0.456)\end{array}$ \\
\hline Husband is unemployed $*$ October 2008 & $\begin{array}{c}0.456^{* *} \\
(0.173)\end{array}$ & $\begin{array}{c}0.690 \\
(0.361)\end{array}$ & $\begin{array}{c}0.546 \\
(0.202)\end{array}$ & $\begin{array}{c}0.639 \\
(0.335)\end{array}$ \\
\hline Husband is unemployed ${ }^{*}$ November 2008 & $\begin{array}{c}0.915 \\
(0.406)\end{array}$ & $\begin{array}{l}1.215 \\
(0.548)\end{array}$ & $\begin{array}{l}1.060 \\
(0.474)\end{array}$ & $\begin{array}{c}1.154 \\
(0.536)\end{array}$ \\
\hline Husband is unemployed ${ }^{*}$ January 2009 & $\begin{array}{c}0.894 \\
(0.350)\end{array}$ & $\begin{array}{l}1.007 \\
(0.401)\end{array}$ & $\begin{array}{c}0.990 \\
(0.397)\end{array}$ & $\begin{array}{c}0.998 \\
(0.404)\end{array}$ \\
\hline Husband is unemployed ${ }^{*}$ February 2009 & $\begin{array}{l}0.499 * \\
(0.177)\end{array}$ & $\begin{array}{c}0.969 \\
(0.500)\end{array}$ & $\begin{array}{c}0.545 \\
(0.201)\end{array}$ & $\begin{array}{c}0.971 \\
(0.491)\end{array}$ \\
\hline Husband is unemployed ${ }^{*}$ March 2009 & $\begin{array}{c}0.811 \\
(0.332)\end{array}$ & $\begin{array}{c}2.611 * * * \\
(0.787)\end{array}$ & $\begin{array}{c}0.876 \\
(0.354)\end{array}$ & $\begin{array}{c}2.570^{* * *} \\
(0.774)\end{array}$ \\
\hline Husband is unemployed*April2009 & $\begin{array}{c}0.515 * * \\
(0.164)\end{array}$ & $\begin{array}{c}2.178 * * \\
(0.770)\end{array}$ & $\begin{array}{l}0.549 * \\
(0.179)\end{array}$ & $\begin{array}{l}2.185 * * \\
(0.763)\end{array}$ \\
\hline Husband is unemployed ${ }^{*}$ May 2009 & $\begin{array}{c}0.772 \\
(0.312)\end{array}$ & $\begin{array}{c}1.558 \\
(0.653)\end{array}$ & $\begin{array}{c}0.783 \\
(0.329)\end{array}$ & $\begin{array}{c}1.543 \\
(0.669)\end{array}$ \\
\hline
\end{tabular}


Husband is unemployed ${ }^{*} J u n e 2009$

Husband is unemployed $*$ July2009

Husband is unemployed*August2009

Husband is unemployed $*$ September 2009

Husband is unemployed ${ }^{*}$ October 2009

Husband is unemployed*November2009

Husband is unemployed ${ }^{*}$ December 2009

Husband is unemployed*January 2010

Husband is unemployed*February 2010

Husband is unemployed*March2010

Husband is unemployed *April2010

Husband is unemployed*May2010

Husband is unemployed ${ }^{*} J u n e 2010$

Husband is unemployed ${ }^{*} J u l y 2010$

Husband is unemployed ${ }^{*}$ August 2010

Husband's education (ref: some college)

Less than high school

High school graduate

Bachelor degree

Masters or higher

Husband's age

Husband's unemployment insurance (ref: no ins.)

Wife's characteristics

Wife's education (ref: some college)

Less than high school

High school graduate

Bachelor degree

Masters or higher

Wife's age

Wife's age square ${ }^{(a)}$

Wife's race \& ethnicity (ref: White alone)

Black

Asian

Other races or more than one

Family variables

Number of children under 18 (ref: 2 children) 0 child

1 child

3 or more children

Residence (ref: metro)

Not metro

Not Identified

Unemployment (state)

Observations

184,424

\begin{tabular}{|c|c|c|c|}
\hline $\begin{array}{c}1.494 \\
(0.860)\end{array}$ & $\begin{array}{l}2.084 * \\
(0.862)\end{array}$ & $\begin{array}{c}1.524 \\
(0.882)\end{array}$ & $\begin{array}{l}2.128^{*} \\
(0.934)\end{array}$ \\
\hline 0.592 & $3.304 * * *$ & 0.613 & $3.316^{* * *}$ \\
\hline$(0.206)$ & $(1.171)$ & $(0.221)$ & (1.199) \\
\hline 0.726 & 0.961 & 0.762 & 0.944 \\
\hline$(0.259)$ & $(0.433)$ & $(0.287)$ & $(0.433)$ \\
\hline $0.529^{*}$ & 1.005 & 0.556 & 0.983 \\
\hline$(0.193)$ & $(0.475)$ & $(0.207)$ & $(0.482)$ \\
\hline 1.299 & 0.424 & 1.335 & 0.424 \\
\hline$(0.500)$ & $(0.291)$ & $(0.508)$ & $(0.288)$ \\
\hline 0.577 & $1.949^{*}$ & 0.610 & $1.961^{*}$ \\
\hline$(0.237)$ & $(0.741)$ & $(0.263)$ & $(0.791)$ \\
\hline 1.055 & $1.973 *$ & 1.127 & $1.983^{*}$ \\
\hline$(0.424)$ & $(0.755)$ & $(0.475)$ & $(0.793)$ \\
\hline 0.674 & $2.182 *$ & 0.743 & $2.108^{*}$ \\
\hline$(0.208)$ & $(0.867)$ & $(0.239)$ & $(0.862)$ \\
\hline 0.604 & 1.782 & 0.686 & 1.715 \\
\hline$(0.216)$ & (0.694) & $(0.251)$ & $(0.669)$ \\
\hline 0.839 & $1.686 *$ & 0.914 & 1.596 \\
\hline$(0.300)$ & $(0.529)$ & $(0.322)$ & $(0.519)$ \\
\hline 0.895 & 0.870 & 0.985 & 0.829 \\
\hline$(0.439)$ & $(0.573)$ & $(0.500)$ & $(0.558)$ \\
\hline 0.638 & 1.101 & 0.689 & 1.067 \\
\hline$(0.200)$ & $(0.546)$ & $(0.217)$ & $(0.538)$ \\
\hline 0.599 & $2.074 *$ & 0.636 & $2.032 *$ \\
\hline$(0.229)$ & $(0.815)$ & $(0.247)$ & $(0.794)$ \\
\hline 1.054 & 0.404 & 1.106 & 0.383 \\
\hline$(0.574)$ & $(0.279)$ & $(0.615)$ & $(0.259)$ \\
\hline 0.844 & 0.991 & 0.883 & 0.941 \\
\hline \multirow[t]{43}{*}{$(0.420)$} & $(0.351)$ & $(0.438)$ & $(0.342)$ \\
\hline & & $0.739 * * *$ & 0.982 \\
\hline & & $(0.074)$ & $(0.095)$ \\
\hline & & 0.950 & 1.106 \\
\hline & & $(0.063)$ & $(0.089)$ \\
\hline & & 1.076 & $0.685^{* * *}$ \\
\hline & & $(0.080)$ & $(0.059)$ \\
\hline & & $0.756 * * *$ & $0.576^{* * *}$ \\
\hline & & $(0.080)$ & $(0.060)$ \\
\hline & & 1.002 & 1.004 \\
\hline & & $(0.006)$ & $(0.006)$ \\
\hline & & 1.146 & 0.918 \\
\hline & & $(0.168)$ & $(0.128)$ \\
\hline & & $0.588 * * *$ & $0.673^{* * *}$ \\
\hline & & $(0.055)$ & $(0.079)$ \\
\hline & & $0.826 * * *$ & $0.825 * * *$ \\
\hline & & $(0.050)$ & $(0.058)$ \\
\hline & & $1.257 * * *$ & $1.299 * * *$ \\
\hline & & $(0.097)$ & $(0.106)$ \\
\hline & & $1.969 * * *$ & $1.601 * * *$ \\
\hline & & $(0.234)$ & $(0.189)$ \\
\hline & & $1.139 * * *$ & $1.067 *$ \\
\hline & & $(0.038)$ & $(0.040)$ \\
\hline & & $0.874 * * *$ & $0.892 * *$ \\
\hline & & $(0.036)$ & $(0.043)$ \\
\hline & & 0.939 & $1.489^{* * *}$ \\
\hline & & $(0.100)$ & $(0.151)$ \\
\hline & & $0.793 * *$ & $0.750 * * *$ \\
\hline & & $(0.087)$ & $(0.081)$ \\
\hline & & $0.751 * *$ & 1.122 \\
\hline & & $(0.104)$ & $(0.163)$ \\
\hline & & $1.125^{*}$ & $1.602 * * *$ \\
\hline & & $(0.076)$ & $(0.117)$ \\
\hline & & 0.955 & $1.220^{* * *}$ \\
\hline & & $(0.062)$ & $(0.089)$ \\
\hline & & $0.798 * * *$ & $0.886^{*}$ \\
\hline & & $(0.061)$ & $(0.060)$ \\
\hline & & 0.978 & 1.032 \\
\hline & & $(0.065)$ & $(0.083)$ \\
\hline & & 1.074 & 1.097 \\
\hline & & $(0.160)$ & $(0.164)$ \\
\hline & & 0.979 & 0.985 \\
\hline & & $(0.014)$ & $(0.016)$ \\
\hline 184,424 & 63,793 & 184,424 & 63,793 \\
\hline
\end{tabular}


Notes: Standard errors in parentheses. $* * * \mathrm{p}<0.01, * * \mathrm{p}<0.05, * \mathrm{p}<0.1$

${ }^{(a)}$ Wife's age square is defined by age square divided by hundred.

Table A10. Average partial effect predicting wives' likelihood of labor force participation at $t$ as function of husband's labor force status in prior month(s), 2008 to 2010.

\begin{tabular}{|c|c|c|c|c|c|}
\hline & (1) & (2) & (3) & (4) & (5) \\
\hline & $\begin{array}{c}\text { wife in lf in } \\
(t-1)\end{array}$ & $\begin{array}{c}\text { wife not in lf } \\
\text { in }(t-1)\end{array}$ & $\begin{array}{l}\text { wife in lf or nlf } \\
\text { in }(t-1)\end{array}$ & $\begin{array}{c}\text { wife in lf in } \\
(t-1)\end{array}$ & $\begin{array}{c}\text { wife not in lf } \\
\text { in }(t-1)\end{array}$ \\
\hline \multicolumn{6}{|l|}{ Husband's characteristics } \\
\hline \multicolumn{6}{|l|}{ Husband's labor force status (ref: hus is emp at $t-1$ ) } \\
\hline Husband is unemployed at $(\mathrm{t}-1)$ & $\begin{array}{l}-0.003 * \\
(0.002)\end{array}$ & $\begin{array}{c}0.015^{* * *} \\
(0.005)\end{array}$ & $\begin{array}{c}0.029 * * * \\
(0.011)\end{array}$ & $\begin{array}{l}-0.004 \\
(0.003)\end{array}$ & $\begin{array}{c}0.011 \\
(0.007)\end{array}$ \\
\hline \multirow[t]{2}{*}{ Husband not in labor force at $(\mathrm{t}-1)$} & 0.001 & $0.011 * *$ & -0.003 & 0.001 & $0.013 * * *$ \\
\hline & $(0.001)$ & $(0.005)$ & $(0.012)$ & $(0.001)$ & $(0.005)$ \\
\hline \multirow[t]{2}{*}{ Husband unemployed both in (t-1) \& (t-2) } & & & & 0.001 & 0.002 \\
\hline & & & & $(0.002)$ & $(0.007)$ \\
\hline \multicolumn{6}{|l|}{ Husband's education (ref: some college) } \\
\hline Less than high school & $\begin{array}{c}-0.004 * * * \\
(0.001)\end{array}$ & $\begin{array}{l}-0.001 \\
(0.004)\end{array}$ & $\begin{array}{c}-0.053 * * * \\
(0.014)\end{array}$ & $\begin{array}{c}-0.004 * * * \\
(0.002)\end{array}$ & $\begin{array}{l}-0.001 \\
(0.004)\end{array}$ \\
\hline \multirow[t]{2}{*}{ High school graduate } & -0.001 & 0.004 & 0.013 & -0.001 & 0.004 \\
\hline & $(0.001)$ & $(0.004)$ & $(0.009)$ & $(0.001)$ & $(0.004)$ \\
\hline \multirow[t]{2}{*}{ Bachelor degree } & 0.001 & $-0.013 * * *$ & $-0.052 * * *$ & 0.001 & $-0.013 * * *$ \\
\hline & $(0.001)$ & $(0.003)$ & $(0.011)$ & $(0.001)$ & $(0.003)$ \\
\hline \multirow[t]{2}{*}{ Masters or higher } & $-0.004 * *$ & $-0.018 * * *$ & $-0.159 * * *$ & $-0.004 * *$ & $-0.017 * * *$ \\
\hline & $(0.002)$ & $(0.003)$ & $(0.015)$ & $(0.002)$ & $(0.003)$ \\
\hline \multirow[t]{2}{*}{ Husband's age } & 0.000 & 0.000 & 0.000 & 0.000 & 0.000 \\
\hline & $(0.000)$ & $(0.000)$ & $(0.001)$ & $(0.000)$ & $(0.000)$ \\
\hline \multirow{2}{*}{ Husband's unemployment insurance (ref: no ins.) } & 0.001 & -0.003 & 0.007 & 0.001 & -0.002 \\
\hline & $(0.002)$ & $(0.004)$ & $(0.013)$ & $(0.002)$ & $(0.005)$ \\
\hline \multicolumn{6}{|l|}{ Wife's characteristics } \\
\hline \multicolumn{6}{|l|}{ Wife's education (ref: some college) } \\
\hline \multirow[t]{2}{*}{ Less than high school } & $-0.009 * * *$ & $-0.012 * * *$ & $-0.220 * * *$ & $-0.009^{* * *}$ & $-0.012 * * *$ \\
\hline & $(0.002)$ & $(0.003)$ & $(0.020)$ & $(0.002)$ & $(0.004)$ \\
\hline \multirow[t]{2}{*}{ High school graduate } & $-0.003 * * *$ & $-0.007 * * *$ & $-0.106^{* * *}$ & $-0.003 * * *$ & $-0.006^{* *}$ \\
\hline & $(0.001)$ & $(0.002)$ & $(0.010)$ & $(0.001)$ & $(0.002)$ \\
\hline \multirow[t]{2}{*}{ Bachelor degree } & $0.003 * * *$ & $0.011 * * *$ & $0.048 * * *$ & $0.003 * * *$ & $0.010 * * *$ \\
\hline & $(0.001)$ & $(0.004)$ & $(0.010)$ & $(0.001)$ & $(0.004)$ \\
\hline Masters or higher & $0.006 * * *$ & $0.022 * * *$ & $0.132 * * *$ & $0.006^{* * *}$ & $0.021 * * *$ \\
\hline & $(0.001)$ & $(0.006)$ & $(0.011)$ & $(0.001)$ & $(0.007)$ \\
\hline Wife's age & $0.002 * * *$ & $0.003 *$ & $0.029 * * *$ & $0.002 * * *$ & $0.003^{*}$ \\
\hline & $(0.000)$ & $(0.001)$ & $(0.006)$ & $(0.000)$ & $(0.001)$ \\
\hline Wife's age square ${ }^{(a)}$ & $-0.002 * * *$ & $-0.004 * *$ & $-0.036^{* * *}$ & $-0.002 * * *$ & $-0.005 * *$ \\
\hline & $(0.000)$ & $(0.002)$ & $(0.007)$ & $(0.001)$ & $(0.002)$ \\
\hline Wife's race \& ethnicity (ref: White alone) & & & & & \\
\hline Black & -0.001 & $0.016 * * *$ & $0.056^{* * *}$ & -0.001 & $0.017 * * *$ \\
\hline & $(0.001)$ & $(0.005)$ & $(0.013)$ & $(0.001)$ & $(0.005)$ \\
\hline Asian & $-0.003 *$ & $-0.009 * * *$ & $-0.091 * * *$ & $-0.003 *$ & $-0.008 * *$ \\
\hline & $(0.002)$ & $(0.003)$ & $(0.018)$ & $(0.002)$ & $(0.003)$ \\
\hline Other races or more than one & $-0.004 *$ & 0.004 & -0.005 & -0.004 & 0.004 \\
\hline & $(0.002)$ & $(0.006)$ & $(0.021)$ & $(0.002)$ & $(0.006)$ \\
\hline Family variables & & & & & \\
\hline Number of children under 18 (ref: 2 children) & & & & & \\
\hline 0 child & $0.001 *$ & $0.019 * * *$ & $0.102 * * *$ & 0.001 & $0.019 * * *$ \\
\hline & $(0.001)$ & $(0.003)$ & $(0.010)$ & $(0.001)$ & $(0.003)$ \\
\hline 1 child & -0.001 & $0.007 * * *$ & $0.038 * * *$ & -0.001 & $0.007 * *$ \\
\hline & $(0.001)$ & $(0.003)$ & $(0.009)$ & $(0.001)$ & $(0.003)$ \\
\hline 3 or more children & $-0.003 * * *$ & $-0.004 *$ & $-0.111 * * *$ & $-0.003 * * *$ & -0.003 \\
\hline & $(0.001)$ & $(0.002)$ & $(0.012)$ & $(0.001)$ & $(0.002)$ \\
\hline Residence (ref: metro) & & & & & \\
\hline Not metro & -0.000 & 0.000 & 0.018 & -0.000 & 0.000 \\
\hline & $(0.001)$ & $(0.003)$ & $(0.012)$ & $(0.001)$ & $(0.003)$ \\
\hline Not Identified & 0.001 & 0.003 & $0.035^{*}$ & 0.001 & 0.001 \\
\hline & $(0.002)$ & $(0.006)$ & $(0.021)$ & $(0.002)$ & $(0.006)$ \\
\hline Unemployment (state) & $-0.001 * * *$ & $-0.001 * * *$ & $-0.004 * * *$ & $-0.000^{* * *}$ & $-0.002 * * *$ \\
\hline & $(0.000)$ & $(0.000)$ & $(0.001)$ & $(0.000)$ & $(0.000)$ \\
\hline Observations & 184,424 & 63,793 & 248,217 & 175,063 & 60,266 \\
\hline
\end{tabular}

Notes: Standard errors in parentheses. ${ }^{* * *} \mathrm{p}<0.01,{ }^{*} \mathrm{p}<0.05,{ }^{*} \mathrm{p}<0.1$

${ }^{(a)}$ Wife's age square is defined by age square divided by hundred. 
Table A11. Means for variables: wife in LF in $t$, husband employed in $(t-1)$, husband unemployed in $(t-1) \&$ husband not in the labor force in $(t-1)$

\begin{tabular}{|c|c|c|c|c|c|c|c|c|}
\hline \multirow[b]{2}{*}{ Month $(t)$} & \multicolumn{4}{|c|}{ Wife in LF in $(t-1)$ : } & \multicolumn{4}{|c|}{ Wife NILF in $(t-1)$ : } \\
\hline & $\begin{array}{l}\text { Wife in } \\
L F \text { in } t\end{array}$ & $\begin{array}{l}\text { Hus emp } \\
\text { in }(t-1)\end{array}$ & $\begin{array}{l}\text { Hus unemp } \\
\text { in }(t-1)\end{array}$ & $\begin{array}{l}\text { Hus NILF } \\
\text { in }(t-1)\end{array}$ & $\begin{array}{l}\text { Wife in } \\
L F \text { in } t\end{array}$ & $\begin{array}{l}\text { Hus emp } \\
\text { in }(t-1)\end{array}$ & $\begin{array}{l}\text { Hus unemp } \\
\text { in }(t-1)\end{array}$ & $\begin{array}{l}\text { Hus NILF } \\
\text { in }(t-1)\end{array}$ \\
\hline Jun- 08 & 0.994 & 0.907 & 0.030 & 0.063 & 0.036 & 0.880 & 0.032 & 0.089 \\
\hline Jul-08 & 0.996 & 0.905 & 0.037 & 0.058 & 0.033 & 0.886 & 0.032 & 0.082 \\
\hline Aug-08 & 0.994 & 0.902 & 0.041 & 0.057 & 0.034 & 0.899 & 0.038 & 0.063 \\
\hline Sep-08 & 0.990 & 0.904 & 0.042 & 0.055 & 0.065 & 0.899 & 0.035 & 0.065 \\
\hline Oct- 08 & 0.990 & 0.909 & 0.040 & 0.051 & 0.053 & 0.903 & 0.038 & 0.059 \\
\hline Nov-08 & 0.989 & 0.908 & 0.044 & 0.047 & 0.040 & 0.898 & 0.047 & 0.055 \\
\hline Dec-08 & 0.989 & 0.904 & 0.048 & 0.049 & 0.042 & 0.887 & 0.056 & 0.057 \\
\hline Jan-09 & 0.985 & 0.888 & 0.062 & 0.050 & 0.038 & 0.876 & 0.066 & 0.058 \\
\hline Feb-09 & 0.988 & 0.882 & 0.066 & 0.053 & 0.033 & 0.885 & 0.062 & 0.053 \\
\hline Mar-09 & 0.987 & 0.884 & 0.064 & 0.052 & 0.032 & 0.873 & 0.072 & 0.055 \\
\hline Apr-09 & 0.990 & 0.882 & 0.066 & 0.052 & 0.041 & 0.878 & 0.067 & 0.055 \\
\hline May-09 & 0.987 & 0.879 & 0.071 & 0.050 & 0.034 & 0.883 & 0.064 & 0.052 \\
\hline Jun-09 & 0.990 & 0.879 & 0.070 & 0.051 & 0.038 & 0.888 & 0.058 & 0.054 \\
\hline Jul-09 & 0.986 & 0.875 & 0.073 & 0.053 & 0.044 & 0.890 & 0.058 & 0.052 \\
\hline Aug-09 & 0.986 & 0.871 & 0.076 & 0.053 & 0.044 & 0.893 & 0.058 & 0.048 \\
\hline Sep-09 & 0.987 & 0.873 & 0.074 & 0.053 & 0.048 & 0.888 & 0.066 & 0.046 \\
\hline Oct-09 & 0.987 & 0.878 & 0.069 & 0.053 & 0.029 & 0.890 & 0.064 & 0.047 \\
\hline Nov-09 & 0.986 & 0.878 & 0.068 & 0.054 & 0.036 & 0.889 & 0.061 & 0.050 \\
\hline Dec-09 & 0.984 & 0.878 & 0.071 & 0.052 & 0.031 & 0.881 & 0.069 & 0.050 \\
\hline Jan-10 & 0.986 & 0.866 & 0.081 & 0.053 & 0.032 & 0.873 & 0.076 & 0.050 \\
\hline Feb-10 & 0.989 & 0.866 & 0.082 & 0.052 & 0.028 & 0.872 & 0.073 & 0.054 \\
\hline Mar-10 & 0.987 & 0.870 & 0.078 & 0.052 & 0.041 & 0.871 & 0.072 & 0.058 \\
\hline Apr-10 & 0.988 & 0.872 & 0.076 & 0.052 & 0.026 & 0.878 & 0.064 & 0.059 \\
\hline May-10 & 0.984 & 0.876 & 0.071 & 0.054 & 0.031 & 0.879 & 0.065 & 0.056 \\
\hline Jun-10 & 0.986 & 0.876 & 0.067 & 0.057 & 0.035 & 0.883 & 0.059 & 0.058 \\
\hline Jul-10 & 0.986 & 0.880 & 0.065 & 0.055 & 0.039 & 0.882 & 0.060 & 0.058 \\
\hline Aug-10 & 0.988 & 0.881 & 0.063 & 0.056 & 0.047 & 0.882 & 0.060 & 0.058 \\
\hline
\end{tabular}

Note: LF and NILF refer to not in labor force respectively. 


\section{CHAPTER 2: THE IMPACT OF MARRIED WOMEN'S LABOR MARKET PARTICIPATION ON POVERTY DURING THE GREAT RECESSION}

\section{Introduction}

Due to a decline in the income share of young and less educated households, poverty in the United States during the great recession rose dramatically and continued to grow until 2012 (Thompson \& Smeeding, 2013). ${ }^{9}$ In response, federal and state governments introduced a variety of social policies, including extending Unemployment Insurance (UI) for up to two years. Households also took some measures to fight poverty. Spouses of unemployed husbands joined the labor force as income from spouses served as insurance in dual-earner families. Males and females suffered differentially in the face of the great recession. Males were impacted more than females in terms of job loss, and the recession was dubbed a "mancession" (Blau \& Winkler, 2017). Working wives' contributions saved some families from falling into poverty or contributed to maintaining consumption smoothing. This study investigates to what extent a working wife helped her family escape poverty and how this function developed over the course of the great recession.

Some studies look at the wife's labor supply as a substitute for a husband's earnings, whereas other studies consider how husbands' and wives' earnings jointly contribute to family welfare (Cancian \& Reed, 2008; Garcia-Escribano, 2004; Hardoy \& Schøne, 2014; Iceland, 2003; Pencavel, 2007). However, only a few of them discuss income loss by the family that can arise due to an economic slowdown. Also, no study

\footnotetext{
${ }^{9}$ According to the official measure, during the great recession, poverty showed an increasing trend from $13.2 \%$ in 2008 to $14.3 \%$ in 2009 . Poverty reached a record high of $15.0 \%$ in 2012 .
} 
relates poverty to the added worker effect (AWE). ${ }^{10}$ The current study looks at family poverty during the great recession considering the effects of wife's work when the husband loses his job. However, I also consider the role that the wife's earnings have on family poverty in the case where the husband is employed or is out of the labor force for an extended period.

The increasing labor force participation by females makes an important contribution to households' income and overall wellbeing. In this paper, I start with the question: What is the impact of married women's labor market participation on poverty during the recession of 2008-2010? The research question is novel, and to the best of my knowledge, no one has addressed this important research question. In this paper, I will report exactly what happens during the great recession in terms of wife's monetary contribution to the family.

Those married women who enter the labor market when their husbands lose their jobs during the recessionary period form what has been termed the added worker effect. There is uncertainty about the labor market participation of married women in the face of a husband's job loss. One group of women joined the labor market as their husbands lose their jobs. Those households have income constraints or other reasons they turn to the labor market. On the other hand, other groups of married women did not join the labor market despite their husbands' job loss. My approach considers these households' experiences and the extent to which they have other resources protecting them from poverty.

\footnotetext{
${ }^{10}$ The added worker effect is defined as the impact on labor supply of wives' who are transitioning to the labor market due to their husbands' unemployment.
} 
The prior literature focuses on the effect of working wives' on income inequality (Agrawal \& Desai, 2017; Betson \& Van Der Gaag, 1984; Cancian \& Reed, 1999; Iceland, 2003; Kimberlin et al., 2016; Sudo, 2017). These studies considered single earners vs. joint earners' households, comparing husbands' and wives' contributions. Cattan (1998) is the only paper that looked at the effect of working wives on poverty, but its results are open to question on methodological grounds. It focuses on descriptive statistics and their changes over time and examines only aggregate relationships between women's labor force participation and poverty. In contrast, this study examines the direct tie between a wife's labor force participation and her family's poverty status.

To summarize main results, overall wife's earnings reduced family poverty level by around 10 percentage points during the great recession and hovered around 30 percentage points for families with unemployed husbands. Families with husbands that are not in the labor force experience the greatest impact from wife's earnings on poverty, as high as 35 percentage points. I also use an extension of the standard Blinder-Oaxaca decomposition framework that decomposes the working wife's effect on the proportion of families below poverty as it changes through the recession. The study uses Official Poverty Measure (OPM) as a conventional and widely used poverty measure and a new Supplemental Poverty Measure (SPM). Replicating the OPM analyses with the SPM does not alter the main findings of the study. Overall, these findings provide evidence that wives' labor market participation works as insurance for low-income families, suggesting that policies to encourage and facilitate women's work will reduce poverty. 
As study findings confirm the importance of wives' employment, they underscore the difficulties that poor families faced during the COVID-19 recession, in which employment for women was particularly hard hit.

\section{Literature Review}

Wives' labor force participation has increased substantially over the decades. Since 1970, the increase has happened mostly for women raising children (Blau, 1998; Blau \& Kahn, 2007). There were changes in the level and distribution of income for married couple families and single female headed families (Cancian \& Reed, 1999). From the perspective of the household, wives' financial contributions to their families have become increasingly important. At the same time, household income inequality is also increasing. Garcia-Escribano (2004) studied the intensive and extensive margins of wives' labor force participation responsiveness due to a transitory income loss of their husbands using PSID data from 1975 to 1991. The study found a strong causality. In particular, in families with credit constraints, wives' were more likely to respond to husband's earnings loss by increasing labor force participation.

Wives' earnings have reduced income inequality in the US from 1979 to 1989. Rejecting the Gini coefficient's standard decomposition, Cancian and Reed (1998, 1999) proposed three counterfactual-based measures that address the research question. In the first measure, the study considered the observed level of inequality relative to what would have been the level of inequality if wives had no income. The second measure considered a reduction of wives' earnings by a small percentage and compared the degree of inequality with the initial level of inequality. In the third measure, the study set the 
income for all wives equal to the observed mean, comparing the degree of inequality with the observed level of inequality. All three approaches suggested that the wives' earnings have reduced family income inequality. The impact of earnings of working married women on the distribution of total household income in the United States from 19681980 shows that there has been an increasing importance of wives' earnings on total household income and served to decrease inequality as well (Betson \& Van Der Gaag, 1984). Increased wives' employment created a substantial reduction in the growth of family earnings inequality, which occurred for highly educated couples (Pencavel, 2007).

Considering the last half of the twentieth century, we observe three contrasting periods of changes. In the first period of 1949 to 1970, income grew dramatically, economic inequality declined, the proportion of single-headed families increased but remained at a low level, and poverty decline sharply (Danziger \& Gottschalk, 1995; Haveman \& Schwabish, 1999). In the second period of 1970 to 1980 , we see slow economic growth coinciding with growing income inequality, while the proportion of single-headed families increased and poverty was stagnant. In the next decade of 1990s, there were mixed trends. Economic growth was at a record high, economic inequality grew, the proportion of single-headed families reached a plateau, and poverty was declining (Cancian \& Reed, 2008; Dalaker, 2001; Jones Jr \& Weinberg, 2000). Overlaid on these trends, an extensive body of research has found that poverty has gone up during the recessions of 1974-1975, 1982-1983, and 1990-1991 (Blank \& Blinder, 1985; Devine \& Wright, 1993; Iceland, 2003b). 


\section{Poverty Measures in the United States}

President Johnson's declaration of the "war on poverty" in 1964 created new momentum in efforts to measure poverty (Fisher, 1992). In the next year, the Office of Economic Opportunity adopted a working definition proposed by Mollie Orshansky. According to this measure, poverty is based on an income threshold of three times the cost of a thrifty food diet to account for other family expenses. The Census Bureau published the first set of poverty estimates in 1967. The Office of Management and Budget directed the Census Bureau to issue poverty estimates beginning in 1969. Since then, there have been numerous minor changes in poverty measure, but the basic structure remains the same (Meyer \& Sullivan, 2012).

The official poverty measure (OPM) is often criticized because it is based on a threshold of income that fails to capture available family resources (Meyer \& Sullivan, 2012). The OPM is based on families' pretax money income, which fails to account for tax liabilities (Social Security and Medicare taxes are particularly important for families with low incomes who have only earnings) and does not include the Earned Income Tax Credit and other tax credits and non-cash benefits, such as food stamps, housing or school lunch subsidies, or public health insurance. A second limitation of the OPM, it is often argued, is that it incorrectly assumes an equivalence for family members regardless their ages. Bishop et al. (2018) find that adding a child and adding an adult to the family are different in terms of their effects on family needs. In addition, when a family has their second child it adds more to the family needs than their first child. From a policy perspective, recent major antipoverty policies are not reflected in the official poverty measure due to growing importance of EITC benefits, Child Tax Credits and Medicaid. 
The growth of the food stamp program (now the Supplemental Nutrition Assistance Program) is also important.

The "Supplemental Poverty Measures (SPM)" published beginning in November 2011, incorporate various changes recommended to the Census Bureau and the Bureau of Labor Statistics. The official poverty estimates are based on pretax cash resources, and the supplemental poverty estimates include noncash benefits from government transfer programs (Short \& Renwick, 2010). Supplemental poverty estimates also subtract certain necessary expenses, like taxes, medical expenses, and housing. Both measures determine a family's poverty status based on the poverty threshold or poverty line, which is the minimum level of resources necessary to meet the basic need of the family. However, they differ by region, as the official poverty measure doesn't consider geographic differences, whereas the supplemental measure accounts for regional differences in living costs. For both measures, cash income includes wages and salaries, interest, dividends, pension payments, Social Security benefits, and cash transfers such as disability payments, unemployment and workers' compensation, and veteran's payments, public assistance, and the like. In addition to cash income the supplemental poverty measure adds government in-kind transfers, including SNAP (food stamps), housing subsidies, low-income home energy assistance, national school lunch program benefits, WIC benefits, etc., and subtracts taxes, child care expenses, expenses related to work, child support paid, and medical expenses. 


\section{Data Collection}

The source of data used in this study is the 2008 Survey of Income and Program Participation conducted by the U.S. Census Bureau. ${ }^{11}$ SIPP is a household-based survey designed as a continuous series of national panels. SIPP has provided interviews of the civilian noninstitutionalized population living in the United States since its inception in 1983 (U.S. Census Bureau, n.d.). The SIPP is based on a nationally representative sample, with households followed for approximately four years and provides data on basic demographic and social characteristics for each member of the household, including age, sex, race, ethnic origin, marital status, relationship within the household, education, and veteran status. The SIPP allows for the examination of the interaction between tax, transfer, and other government and private policies. SIPP collects information on assistance received either directly as cash or indirectly as in-kind benefits.

The structure of the data file is "person-month" and makes available all the data on a monthly basis. Households and their characteristics are defined on a monthly basis. SIPP provides information on labor force status and allows us to track individuals' monthly employment status. Although the SIPP is subject to differential attrition, sample weights are designed to address the bias that may arise from sample attrition (Czajka et al., 2008).

The benefits of using the SIPP data are many. First, it has a shorter recall period than the Current Population Survey (CPS). Respondents need to remember information in only the prior four months, whereas in the CPS respondents must remember 12 months of

\footnotetext{
${ }^{11}$ This study downloaded 2008 SIPP data from the National Bureau of Economic Research (NBER) website where NBER provides Stata data definition files for the convenience of researchers. I use data from this survey from the panel of information on households extending through August 2010. The NBER website is http://www.nber.org/data/survey-of-income-and-program-participation-sipp-data.html.
} 
information. Second, SIPP tracks movers and the CPS does not track movers (Mattingly $\&$ Smith, 2010). Third, SIPP provides weights to make the sample representative of the U.S. population, but as households from high poverty areas are oversampled, there is an increase in the power for analyses, like ours, that focus on households experiencing poverty and unemployment. Fourth, the SIPP survey is particularly focused on obtaining information regarding receipt of government transfers and participation in related programs.

This paper has also estimated poverty based on the supplemental poverty measure (SPM). In addition to the SIPP survey data, I estimate household federal and state income tax liabilities and the federal social insurance taxes using the National Bureau of Economic Research (NBER) TAXSIM simulation model (Feenberg \& Coutts, 1993) based on household income and demographic data from the SIPP survey. Discussion and the procedure to calculate the income tax, which is required to calculate household poverty based on the SPM, can be found in appendix A.

\section{The Sample and Descriptive Statistics Data Collection}

This study uses data from the Survey of Income and Program Participation (SIPP) of 2008. I take 28 months of data from the first seven waves of SIPP 2008, observations for this study extending from May 2008 to August 2010. I discuss two broad areas in this research: socio-economic dynamics of families and how their poverty changes during the great recession.

I have applied some restrictions on the data for this analysis. In the first step, I consider a sample in which husbands and wives were both between 25 and 54 years of 
age. This allows me to remove most students and people who are retired (Cullen \& Gruber, 2000). ${ }^{12}$ In the next step, I limit my sample to married couples who are living together. They are known as "family household married couples."13

In table 1, I present detailed descriptive statistics from the sample. The data are arranged person by month. We have 13,016 households with a total of 261,233 observations. Two broad categories of the sample, households in months with employed husbands and households in months with husbands who are not employed. Husbands not employed include unemployed husbands and husbands who are not in the labor force. In our sample, in about $88 \%$ of months husbands are employed. On average, wives of husbands who are not employed work in $74.6 \%$ of the months in the SIPP data. Wives of husbands who are employed work $74.3 \%$ of the months. Table 1 and all the subsequent analyses in this paper use household weights.

Table 1. Summary statistics.

\begin{tabular}{lcc}
\hline & $\begin{array}{c}\text { Husband employed } \\
(1)\end{array}$ & $\begin{array}{c}\text { Husband not employed } \\
(2)\end{array}$ \\
\hline Wife in labor force & 0.743 & 0.746 \\
Husband's characteristics & 41.9 & 41.3 \\
$\quad$ Husband's age & $(8.1)$ & $(7.7)$ \\
Husband's Unemployment Insurance & $\ldots$ & 0.269 \\
$\quad$ Yes & $\ldots$ & 1156.4 \\
Husband's total earnings & 4963.8 & $(2087.1)$
\end{tabular}

\footnotetext{
${ }^{12}$ There is no consensus about the age of couples for the added worker effect as studies use various age groups for their analyses. For couples, Fernandes and Felício (2005) use ages 25 to 60, Gong (2011) uses ages 22 to 59, Mattingly and Smith (2010) use ages 18 to 65, Parker and Skoufias (2004) use ages 20 to 65, Karaoglan and Okten (2015) use ages 20 to 54, Mankart and Oikonomou (2016) use ages 25 to 55.

${ }^{13}$ Possible household types identified in the SIPP dataset are family married couple, family male householder, family female householder, nonfamily male householder, nonfamily female householder.
} 
Wife not in labor force

Husband total other income

Wife in labor force

Wife not in labor force

Wife's characteristics

Wife's education

Less than high school

High school graduate

Some college

Bachelor degree

Masters or higher

Wife's age

Wives' race and ethnicity

White alone

Black alone

Asian alone

Other races or more than one

Wife total income

Wife in labor force

Wife not in labor force

Wife total other income

Wife in labor force

Wife not in labor force

Family variables

Family income by labor force status

Wife in labor force

Wife not in labor force

Poverty threshold

Wife in labor force

Wife not in labor force

Number of children under 18

0 child

1 child

2 children
5801.3

(6130.3)

1114.3

(2193.7)

49.4

628.1

(386.5)

(976.7)

51.6

700.0

(396.3)

(1015.3)

0.078

0.141

0.199

0.262

0.341

0.346

0.257

0.167

0.126

0.082

39.5

(7.8)

40.0

(8.1)

0.847

0.790

0.069

0.111

0.056

0.059

0.027

0.039

3149.0

2955.7

(3174.8)

(3478.0)

148.6

(542.1)

314.4

(738.4)

63.7

(315.2)

105.3

(394.1)

88.7

222.7

(384.9)

(637.4)

8447.0

4471.1

(4471.1)

1746.0

6233.8

(2497.1)

1687.0

(419.4)

1857.4

(471.0)

1714.2

(513.7)

1796.9

(513.7)

0.308

0.372

0.245

0.237

0.282

0.232 


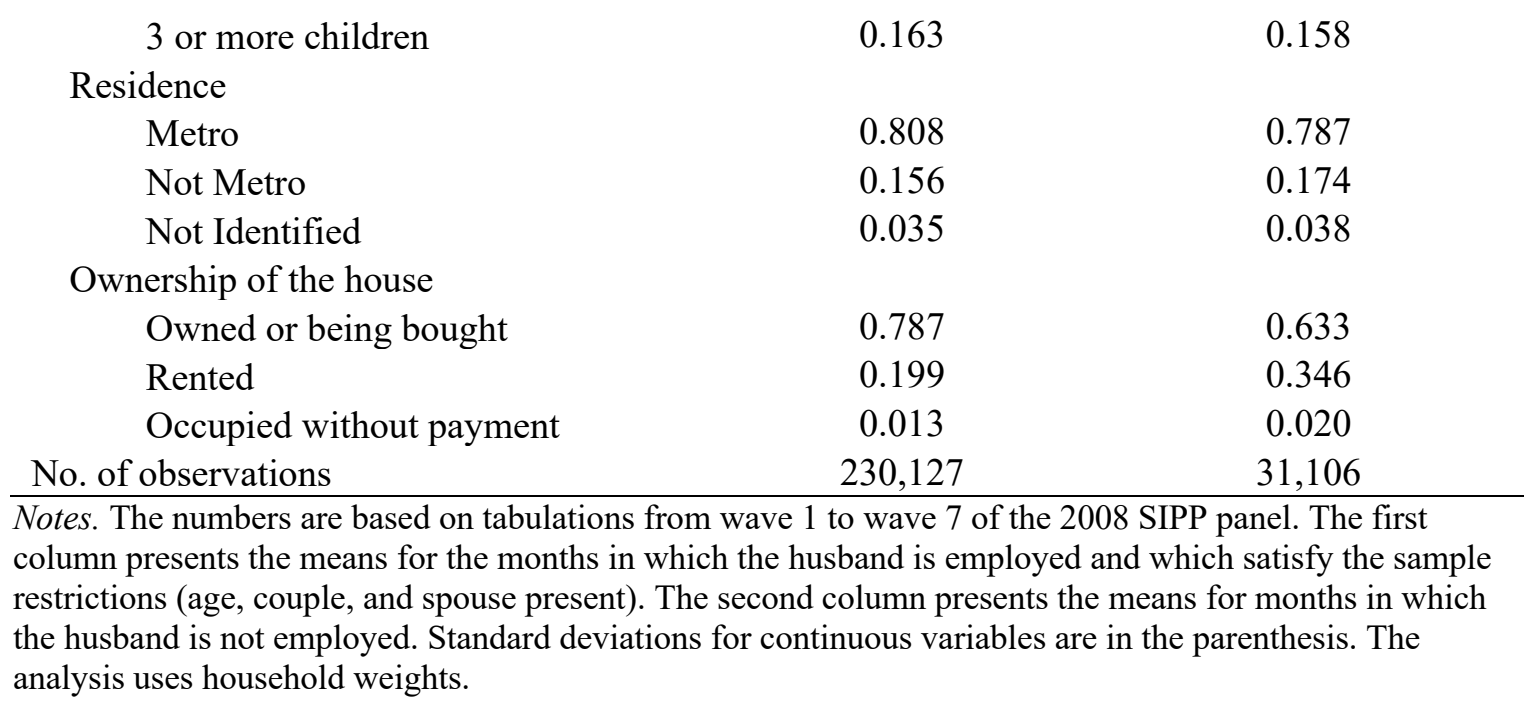

In the SIPP survey, information on all kinds of income is collected for each month with a few exceptions, such as certain kinds of asset income. SIPP earnings information considers three different types of earnings, wages and salary, nonfarm self-employment, and farm self-employment. SIPP also accounts for unearned income including explicit questions for over 30 specific categories, including asset income, unemployment compensation, social security income, and means tested cash transfers. The monthly incomes figures are before deduction of income and payroll taxes, union fees, Medicare premiums, etc. The average monthly family income is $\$ 7,424$ with a standard deviation of $\$ 6,235$ for the observations in 28 months (first seven waves of the SIPP 2008). When we observe monthly family income by labor force status, households where husbands are employed have an average income of $\$ 8,447$, and households where husbands are not employed have an average income of $\$ 4,471$. If we see families where wives' are not in the labor force, the average monthly family incomes are $\$ 6,234$ and $\$ 1,746$ for households with employed husbands and households with husbands who are not employed, respectively. Hence, families, where both husband and wife are employed, 
have an average monthly income that is greater by $383.8 \%$ compared to those families where both husband and wife are not employed (\$8,447 vs. \$1,746).

The SIPP survey provides a monthly poverty threshold for each family. In table 1, we classify the poverty threshold by labor force status of husband and wife, and the average is $\$ 1687$ for families with employed husbands and $\$ 1857$ for families of nonworking husbands. The poverty threshold of a family depends on the number of family members, not the labor force status.

Figure 1 shows the percentage of families in poverty for each calendar month of the survey based on the official poverty measure and identifies the impact of the wife's contribution to family income on poverty. I measure total family income in two different ways. In the first, I include total family income that includes all earned income and other income in order to determine whether the family is below the poverty line. In the second measure, I subtract the wife's earned income from total family income to calculate potential poverty status. In the figure, there are five lines: one line indicates the proportion of families in poverty based on total family income, and four others provide this proportion based on family income after omitting the wife's earnings. The first line from the $\mathrm{x}$-axis (circle markers), based on total family income, shows a dramatic increase of $21.8 \%$ during these 28 months, families below poverty changes from $8.2 \%$ to $10.0 \%$. On average, over the full sample period, $9.3 \%$ of families were below poverty. 
Figure 1. Distribution of poverty by month: percent of family in poverty based on official poverty measure

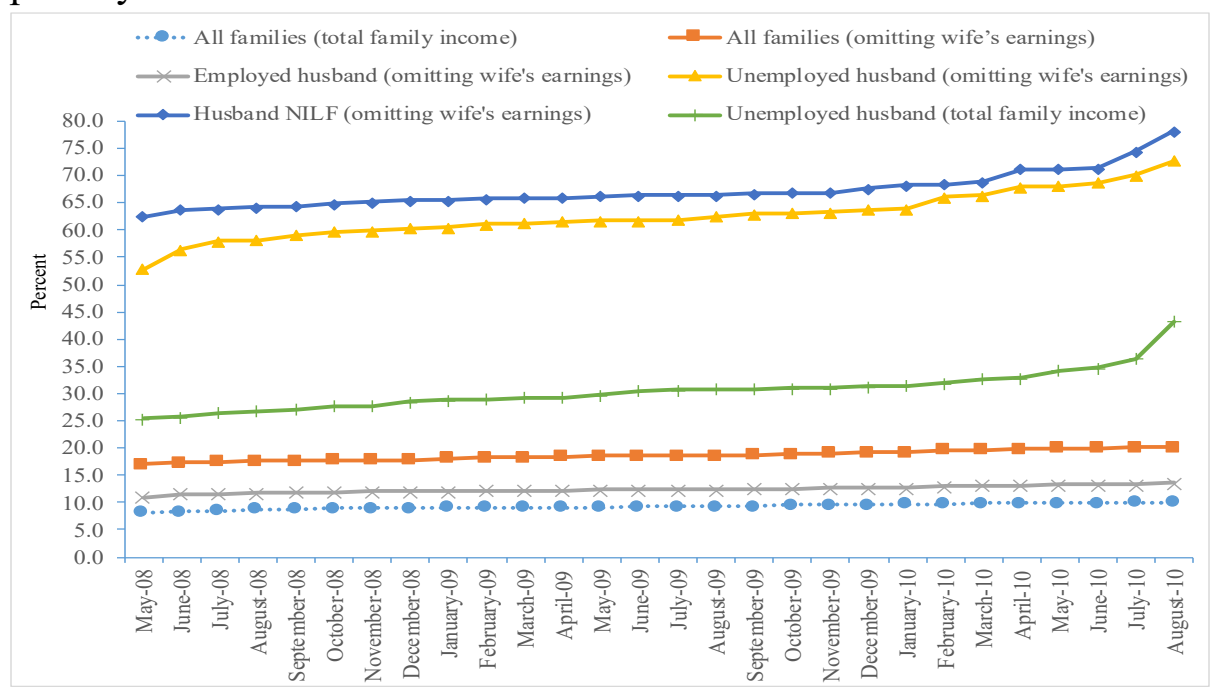

Source. Data analysis from wave 1 to wave 7 of SIPP 2008.

The number of families below poverty is greater in the second half of our data window compared to the first half. This shows that the recession intensifies family poverty. The third line (square markers) is comparable to the first line, as I subtract wife's earnings from total family income. It provides the poverty status of families without the wife's earnings. For this measure, there is an $18.6 \%$ increase in the number of families below poverty during this period, growing from $17.0 \%$ to $20.1 \%$. On average, $18.6 \%$ of families were in poverty over the sample period. It has similar trend as percent of families below poverty with total family income. In the second half of our data window from July 2009 to August 2010, the numbers are greater than the first half from May 2008 to June 2009.

The husbands' labor force status is of central concern for this study. In particular, I focus on unemployed husbands in keeping with the idea of the added worker effect. Three other lines in figure 1 show the proportion of families in poverty by husbands' labor force status. During this period, I can see how poverty would be influenced by 
removing wife's earnings. Removing wives' earnings causes the proportion of families with employed husbands below poverty to grow approximately $26 \%$ (from $10.9 \%$ to 13.7), the proportion with unemployed husbands to grow approximately $38 \%$ (from $52.8 \%$ to 72.7 ), and the proportion with husbands not in the labor force to grow by approximately $25 \%$ (62.5\% to 78.3$)$. Families where husbands are unemployed or not in the labor force constitute the lion's share of family poverty. In the monthly estimates, considering total family income, only $8.2 \%$ of families were in poverty in May 2008, and this rises to $10.0 \%$ in August 2010. After subtracting the wife's earnings from the family's total income, $17.0 \%$ of families had incomes below the poverty line in May 2008, and it rose to $20.1 \%$ in August 2010. These numbers essentially tell us that wives' income has a significant role in reducing the likelihood of poverty for a family.

In both measures of poverty, subtracting wife's earnings has important implications for the level of poverty. For both the OPM and SPM, an additional $9 \%$ of families fall below the poverty line if I omit wife's earnings. The number of families below poverty based on SPM is smaller than the number of families below poverty in the OPM. ${ }^{14}$ The transfer programs likely contribute to this smaller number in SPM compared to the OPM as they increase the measure of family income used under the former measure. For details, see the figure S1 appendix B.

Figure 2 shows the proportion of households in each month by husband's labor force status. In the sample, the percentage of husbands who are unemployed and not in the labor force is about the same. Over our analysis period, on average, $88.1 \%$ of households have employed husbands, $6.2 \%$ of households have unemployed husbands,

\footnotetext{
${ }^{14}$ According to the Census Bureau estimates for the US, the SPM was 1.3 percentage points higher than the OPM of $12.7 \%$ in 2016 (Fox, 2018).
} 
and $5.7 \%$ of households have husbands who are not in the labor force. The increasing trend in the proportion of husbands who are unemployed follows the national pattern, reflecting the recessionary period. However, due to our sample restrictions, the numbers do not correspond to the national statistics. The proportion of unemployed husbands was low at $3.1 \%$ in May 2008, and increases to a maximum of $8.0 \%$ in 2011 . During this period, the percentage of employed husbands decreases. In contrast, the proportion of husbands out of the labor force declines quite quickly in the first six months of our data, and then increases gradually to the end of our data window in August 2010.

Figure 2. Proportion of households in month $t$ with husband labor force status.

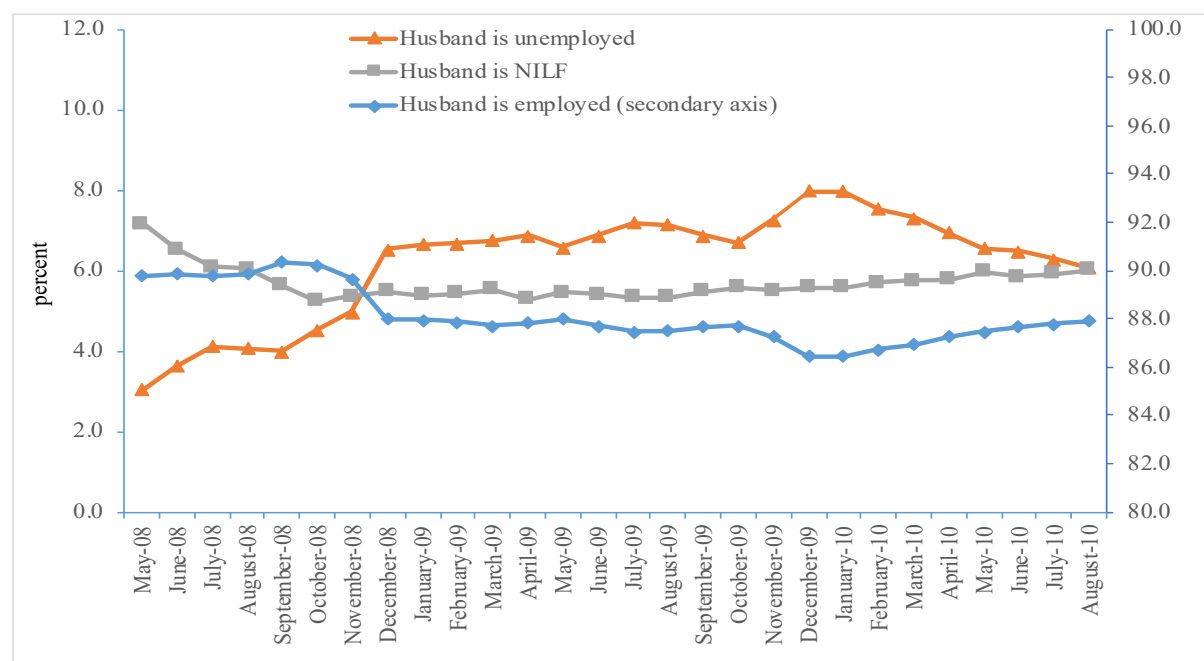

Source. Data analysis from wave 1 to wave 7 of SIPP 2008.

The level of wife's labor force participation as a function of husband's labor force status is also of interest. In the sample period of the great recession, if we compare the proportion of wives in the labor force when the husband is unemployed to that of the case where the husband is not in the labor force, we see they follow similar patterns (figure 3). 
Figure 3. Wife labor force participation (\%)

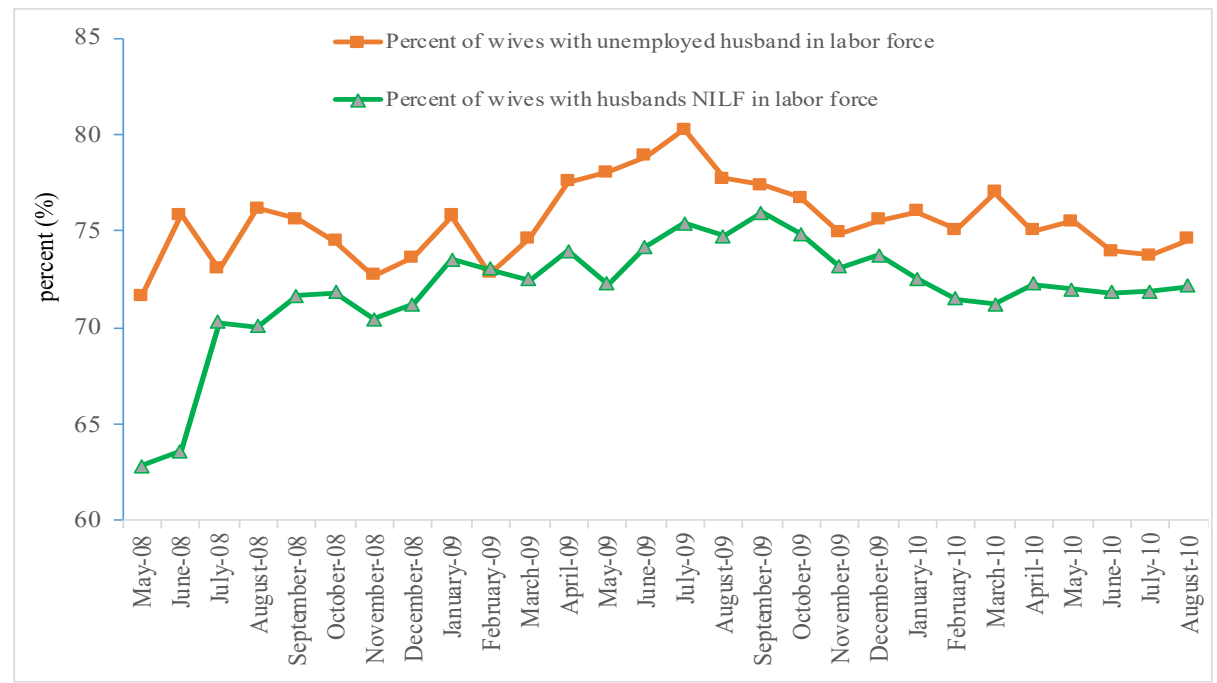

Source. Data analysis from wave 1 to wave 7 of SIPP 2008.

The wife's labor force participation increases initially and reaches a maximum in July 2009 and then decreases. On net, wives were entering the labor market during the first half of the sample period. Throughout the period, wives who have unemployed husbands are more likely to be in the labor force than wives who have husbands who are not in the labor force. On average, $75.5 \%$ of the wives with an unemployed husband were in the labor force, as compared with $71.9 \%$ of wives with husbands who were not in the labor force. This suggests that husbands' unemployment may motivate wives' to enter into the labor market. Employed wives' average earnings followed an uneven but decreasing trend during the great recession through July 2009 and then increased modestly, as shown in figure S2 of appendix B.

Unemployment Insurance benefits were an important source of income for families during the recession as discussed in figure $\mathrm{S} 3$ of appendix B.

In this paper, I am dealing with poverty from a national perspective. However, some state-level poverty data may also be useful to understand that there are regional 
differences. In figure S4 of appendix B, I discussed the relationship between poverty and family income in various states.

\section{Methods}

A family's poverty status depends on family income and family size. Family income can change depending on various factors, but family size is relatively stable. A family's poverty status is affected by the wife's labor force participation, which, in turn, is partly a function of the husband's labor force status. The simplest overall measure of the effect of wife's labor force participation on family poverty status is represented by figure 1. In particular, the percent of families in poverty (circled markers) is compared with the proportion of families in poverty when the wife's earnings are subtracted from the family income (square markers); this gives an exact measure of how many families would move into poverty if wives stopped working and all other sources of income remained unchanged.

The effect of wife's earnings on poverty (in percentage points) is defined as

$$
D P=P_{n w}-P
$$

where $D P$ is the effect of wife's earnings on the level of poverty for families. $P_{n w}$ is the potential poverty level of families based on income after subtracting wife's earnings. $P$ is the poverty level of families considering all income actually received by the families. This approach controls for family characteristics - since the two sets of families are the same ones (hence, they have identical characteristics, both measured and unmeasured). It also fully and completely controls whether the wife is working and how much she earns, since, for families where she is not working, the family's income is the same in the two 
cases. When she is working, the family's income changes by exactly the amount she earns. This measure of wife's impact on poverty changes across calendar months, as the recession deepens. We may expect an increasing trend over months due to the importance of the wife's income in a recession.

The above approach only considers women who choose to work, identifying what would happen to their families if they withdrew from the labor force. It does not answer the question of what would happen to poverty if nonworking women were induced to enter the labor market as it only captures the impact of women who are working in the current labor market.

There is one potential source of bias in the above measure. It does not allow for the possibility that some other income source, for example the husband's earnings (or his employment status), would change in response to his wife's working (or not working). In particular, when we subtract out the wife's earnings, we implicitly assume the amount of other income would not change enough to move the family into or out of poverty.

We use the same principle as described above to calculate the effect of the wife's working on family poverty contingent on the husband's labor market status. The effect of wife's earnings on the poverty level of the family considering the fact that her husband was unemployed is defined as

$$
D P_{h u}=P_{h u, n w}-P_{h u} \ldots \ldots \ldots \ldots \ldots \text { (2) }
$$

where $D P_{h u}$ identifies the effect of wife's earnings on the poverty level of families where the husband was unemployed. $P_{h u, n w}$ defines the proportion of families in poverty, where husbands were unemployed and subtracts wife's earnings from total family income. $P_{h u}$ is the proportion of families in poverty with unemployed husbands considering all 
income including wife's earnings. As in the case above, the approach completely controls for family characteristics (both are the same families), and it fully controls for the wife's actual earnings. As above, the only potential bias is that when the wife works, it may affect some other source of income.

We can similarly calculate the effect of wife's working in the case of the other two husbands' labor force statuses. Using our notation above, for the husband not in the labor force (NILF), we can write

$$
D P_{h n}=P_{h n, n w}-P_{h n}
$$

where $D P_{h n}$ defines the effect of wife's earnings on the proportion of families in poverty where the husband was not in the labor force. $P_{h n, n w}$ is the proportion in poverty when the husband was not in the labor force, and wife's earnings are subtracted from family income. $P_{h n}$ the is the proportion of families in poverty when husband was not in the labor force but considering all income for the family. For employed husbands, we write

$$
D P_{h e}=P_{h e, n w}-P_{h e}
$$

where $D P_{h e}$ defines the effect of wife's earnings on the proportion of families in poverty when the husband was employed. $P_{h e, n w}$ is the proportion of families in poverty when the husband was employed but excluding the wife's earnings. $P_{h e}$ is the level of poverty in the family when husband was employed including all income in the family.

In any month $t$, for these households, the husband must be either unemployed, not in the labor force, or employed, so we can write

$$
D P(t)=p_{h u}(t) * D P_{h u}(t)+p_{h n}(t) * D P_{h n}(t)+p_{h e}(t) * D P_{h e}(t)
$$

where $p_{h u}(t)$ is the proportion of households in month $t$ where the husband is unemployed, $p_{h n}(t)$ is the proportion of households in month $t$ where the husband is not 
in the labor force, and $p_{h e}(t)$ is the proportion of households with employed husbands in month $t$.

An extension of the standard Blinder-Oaxaca decomposition framework can be used to decompose changes in the effect of a working wife on the proportion of families below poverty. ${ }^{15}$ We have three categories of husband: employed, unemployed, and not in the labor force. The mean outcome of the effect of a working wife on poverty can be decomposed into the following five components: that due to the change in the effect of a working wife in households with unemployed husbands, that due to a change in the effect of a working wife in households with husbands not in the labor force, and that due to a change in the effect of a working wife in households with employed husbands, as well as that due to change in proportion of unemployed husband, and that due to change in proportion of NILF husbands.

Focusing on the first term on the right in equation (5), write the change of poverty between two months ( $t$ and $\left.t^{\prime}\right)$ as,

$$
\begin{gathered}
p_{h u}(t) * D P_{h u}(t)-p_{h u}\left(t^{\prime}\right) * D P_{h u}\left(t^{\prime}\right) \\
=\left[p_{h u}(t)+p_{h u}\left(t^{\prime}\right)\right] / 2\left[D P_{h u}(t)-D P_{h u}\left(t^{\prime}\right)\right]+\left[p_{h u}(t)-p_{h u}\left(t^{\prime}\right)\right]\left[D P_{h u}(t)\right. \\
\left.\quad+D P_{h u}\left(t^{\prime}\right)\right] / 2 \ldots \ldots \ldots(6)
\end{gathered}
$$

The expression (6) (the last two lines) decomposes the change $p_{h u}(t) D P_{h u}(t)-p_{h u}\left(t^{\prime}\right) D P_{h u}\left(t^{\prime}\right)$ into a component that reflects the change in $D P_{h u}$ and a component that is due to the change in $p_{h u}$ (i.e., the second of the two lines).

A similar expression can be written out for the change in $p_{h n} * D P_{h n}$, and for $p_{h e} * D P_{h e}$. We can then write out the change in the whole expression as due to six parts:

\footnotetext{
${ }^{15}$ The standard decomposition is most commonly used to decompose differences in earnings for two groups based on factors predicting earnings (Blinder, 1973; Oaxaca, 1973).
} 


$$
\begin{aligned}
& D P(t)-D P\left(t^{\prime}\right) \\
& =p_{h u}(t) * D P_{h u}(t)+p_{h n}(t) * D P_{h n}(t)+p_{h e}(t) * D P_{h e}(t)-p_{h u}\left(t^{\prime}\right) * D P_{h u}\left(t^{\prime}\right) \\
& \quad-p_{h n}\left(t^{\prime}\right) * D P_{h n}\left(t^{\prime}\right)-p_{h e}\left(t^{\prime}\right) * D P_{h e}\left(t^{\prime}\right)
\end{aligned}
$$

But there is one problem with the above, which is that $p_{h u}+p_{h n}+p_{h e}=1$, so these six components are not independent.

One way to solve this problem is to take changes in husband's unemployment status $(H U)$ and not in the labor force status $(H N)$ as relative to employment $(H E)$. We can do this by substituting for $p_{h e}=1-p_{h u}+p_{h n}$. If we do this and rearrange, then we can get

$$
\begin{aligned}
& \text { Equation Interpretation } \\
& D P(t)-D P\left(t^{\prime}\right)= \\
& {\left[p_{h u}(t)+p_{h u}\left(t^{\prime}\right)\right] / 2\left[D P_{h u}(t)-D P_{h u}\left(t^{\prime}\right)\right] \quad \text { (1) due to change in effect of wife's work for }} \\
& \text { families with unemployed husbands } \\
& +\left[p_{h n}(t)+p_{h n}\left(t^{\prime}\right)\right] / 2\left[D P_{h n}(t)-D P_{h n}\left(t^{\prime}\right)\right] \quad \text { (2) due to change in effect of wife's work for } \\
& \text { families with NILF husbands } \\
& +\left[p_{h e}(t)+p_{h e}\left(t^{\prime}\right)\right] / 2\left[D P_{h e}(t)-D P_{h e}\left(t^{\prime}\right)\right] \quad \text { (3) due to change in effect of wife's work for } \\
& \text { families with for employed husbands } \\
& +\left[p_{h u}(t)-p_{h u}\left(t^{\prime}\right)\right]\left\{\frac{\left[D P_{h u}(t)+D P_{h u}\left(t^{\prime}\right)\right]}{2} \quad\right. \text { (4) due to change in proportion of unemployed } \\
& \text { husbands } \\
& \left.-\frac{\left[D P_{h e}(t)+D P_{h e}\left(t^{\prime}\right)\right]}{2}\right\} \\
& +\left[p_{h n}(t)-p_{h n}\left(t^{\prime}\right)\right]\left\{\frac{\left[D P_{h n}(t)+D P_{h n}\left(t^{\prime}\right)\right]}{2} \quad\right. \text { (5) due to change in proportion of NILF husbands } \\
& \left.-\frac{\left[D P_{h e}(t)+D P_{h e}\left(t^{\prime}\right)\right]}{2}\right\}
\end{aligned}
$$


There is no factor identifying the effect of a change in the proportion of employed husbands. The reason is that we have assumed that when we change the proportion unemployed, we automatically change the proportion of employed husbands by that same (opposite) amount; and that when we change the proportion of NILF husbands, we change the proportion of employed husbands by the same (opposite) amount.

This standard decomposition is a variation on the Oaxaca-Blinder decomposition. But it is modified to allow for three sources of change, and to recognize the "adding up" constraint on the proportions.

\section{Results}

The effect of wife's earnings on family poverty is defined by equation 1 in the previous section as the difference between the percent of households in poverty taking account of total family income and the percent of households in poverty omitting wife's earnings. The results are provided in figure 4 . The effect of wife's earnings ranges between 8.7 to 10.1 percentage points based on the official poverty measure. The average impact is 9.4 percentage points. Due to wife's earnings, approximately $10 \%$ of households are out of poverty. Month-to-month variation in the effect of wife's earnings is relatively small during the great recession represented by the square marker and follows a rising trend. The effect of wife's earnings increases by $15.6 \%$ from May 2008 to August 2010. Without wife's income, household poverty would be substantially greater, increasing from about $9 \%$ to about $18 \%$. 
Figure 4. Effect of wife's earnings on the level of poverty based on official poverty

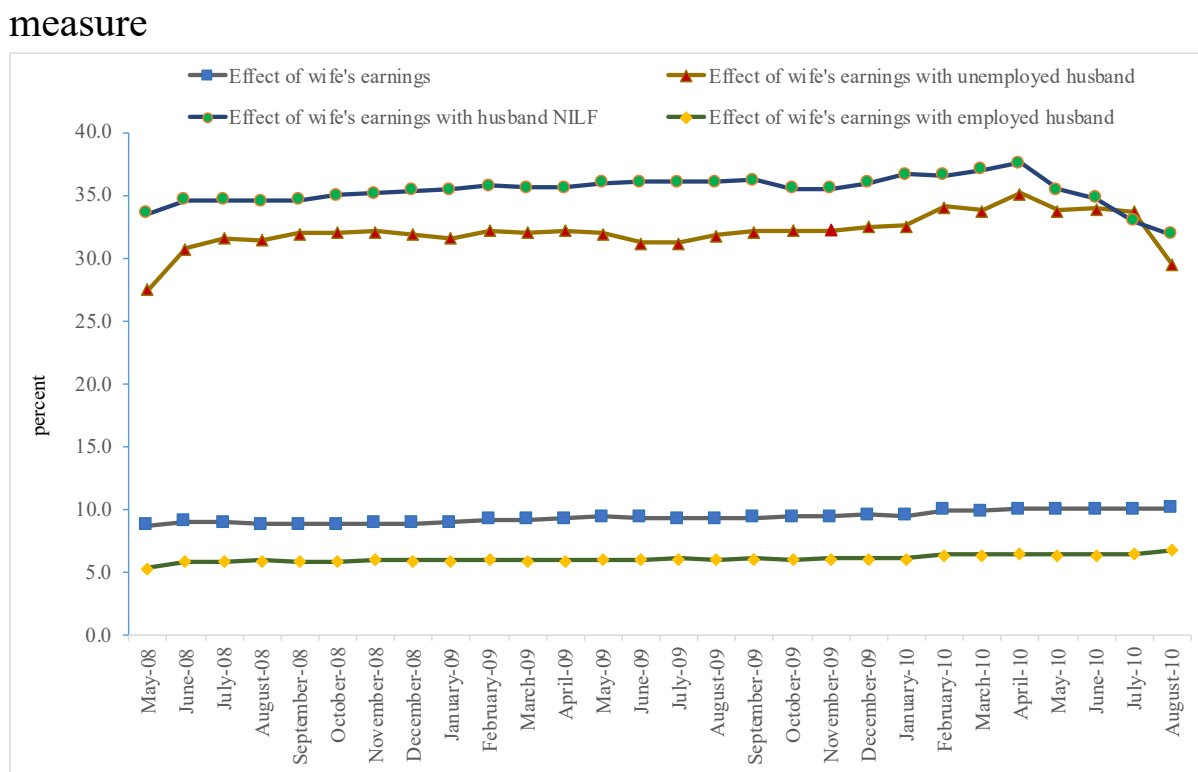

Source. Data analysis from wave 1 to wave 7 of SIPP 2008.

The effect of wife's earnings on the level of poverty where the husband is unemployed is much larger than for the population as a whole. The average effect is 32.1 percentage points for the sample period. This tells us that for families where the husband is unemployed, the proportion of families below the poverty line declines by more than a quarter as a result of wife's earnings. The triangle marker presents the effect of a working wife on family poverty for families where husbands are unemployed. In general, we find an increasing trend of this impact until the last six months of our analysis window, when it declines precipitously. There are two possibilities to explain this decline after April 2010. The proportion of wives in the labor market may be decreasing, or the average income for working wives may be decreasing. It appears that the first explanation is the primary reason, as figure 3 shows that wives are less likely to be in the labor market, whereas figure S2 implies that average earnings for those wives who are working are not changing. 
The effect of wife's earnings for families with a husband who was not in the labor force is slightly larger than for families with unemployed husbands. On average, the effect is 35.4 percentage points, represented by the circled marker. This reflects the fact that wives with husbands not in the labor force earn more than wives with unemployed husbands (results not presented). This more than compensates for the fact that wives are more likely to hold jobs when their husbands are unemployed, as described in figure 3 .

The effect on the level of household poverty of wife's earnings where the husband is employed is smaller than that for families where the husband is not employed. On average, the effect is 6.1 percentage points, represented by the diamond marker. We expect a low impact of a working wife when the husband is working, since most employed husbands earn enough to keep the family above the poverty line even without a wife's earnings. A wife's earnings can give some flexibility to the family in its consumption expenditures, but it is unlikely to be important in meeting the family's basic requirements.

When I replicated these analyses using the supplemental poverty measure (SPM), I also see that the wife's monetary contribution plays an important role in family poverty reduction (figure S5 of appendix B). The basic patterns are very similar to those reported above. One exception is that, according to the official poverty measure (OPM), wife's earnings have an increasing importance over our study period, but that increase is much smaller in the SPM. This is likely to be a reflection of the increasing reliance on government in-kind transfer programs during the recession. In fact, if we omit SNAP payments from the SPM calculation, we discover that the difference in trends largely disappears. 
Table 2 shows the OPM decomposition of the effect of wife's earnings by husband's labor force status based on the methodology developed in equation 7. The change in the effect of wife's working on poverty can be factored into five different components. Monthly values are calculated relative to May $2008\left(t^{\prime}\right)$ in the decomposition. The numbers in the total change in poverty due to wife's earnings in the last column are the difference between the impact of wife's earnings on poverty in the month in question relative to May $2008\left(t^{\prime}\right)$. For example, the number is 1.32 in February 2009, and this is the difference in the impact of wife's work on the poverty level in February 2009 relative to May 2008. The poverty level in May 2008 was 16.94 (having a working wife decreases the poverty level by 16.94 percentage points) and that increases to 18.26 percentage points in February 2009.

Table 2. Poverty decomposition $\left(t^{\prime}<t\right)$ for each month compare to first month $\left(t^{\prime}\right.$ : May 2008), Official Poverty Measure (OPM).

\begin{tabular}{|l|c|c|c|c|c|c|}
\hline $\begin{array}{c}\text { Month \& } \\
\text { Year }\end{array}$ & $\begin{array}{c}\text { Due to } \\
\text { change for } \\
\text { unemployed } \\
\text { husbands }\end{array}$ & $\begin{array}{c}\text { Due to } \\
\text { change for } \\
\text { NILF } \\
\text { husbands }\end{array}$ & $\begin{array}{c}\text { Due to } \\
\text { change for } \\
\text { employed } \\
\text { husbands }\end{array}$ & $\begin{array}{c}\text { Due to } \\
\text { change in } \\
\text { proportion } \\
\text { of } \\
\text { unemployed } \\
\text { husband }\end{array}$ & $\begin{array}{c}\text { Due to } \\
\text { change in } \\
\text { proportion } \\
\text { of NILF } \\
\text { husbands }\end{array}$ & $\begin{array}{c}\text { Total } \\
\text { change in } \\
\text { poverty due } \\
\text { to wife's } \\
\text { earnings }\end{array}$ \\
\hline June-08 & 0.11 & 0.07 & 0.46 & 0.14 & -0.19 & 0.59 \\
\hline July-08 & 0.14 & 0.07 & 0.50 & 0.25 & -0.30 & 0.67 \\
\hline August-08 & 0.14 & 0.06 & 0.55 & 0.24 & -0.32 & 0.67 \\
\hline September-08 & 0.16 & 0.06 & 0.51 & 0.23 & -0.44 & 0.52 \\
\hline October-08 & 0.17 & 0.09 & 0.49 & 0.36 & -0.55 & 0.55 \\
\hline November-08 & 0.18 & 0.10 & 0.63 & 0.47 & -0.52 & 0.86 \\
\hline December-08 & 0.21 & 0.11 & 0.57 & 0.83 & -0.49 & 1.24 \\
\hline January-09 & 0.20 & 0.12 & 0.53 & 0.86 & -0.51 & 1.19 \\
\hline February-09 & 0.23 & 0.13 & 0.59 & 0.87 & -0.50 & 1.32 \\
\hline March-09 & 0.22 & 0.13 & 0.57 & 0.89 & -0.48 & 1.34 \\
\hline April-09 & 0.23 & 0.12 & 0.57 & 0.93 & -0.54 & 1.31 \\
\hline May-09 & 0.21 & 0.15 & 0.59 & 0.85 & -0.50 & 1.30 \\
\hline June-09 & 0.18 & 0.15 & 0.63 & 0.91 & -0.51 & 1.36 \\
\hline July-09 & 0.19 & 0.15 & 0.65 & 0.98 & -0.53 & 1.43 \\
\hline August-09 & 0.22 & 0.15 & 0.58 & 0.98 & -0.53 & 1.40 \\
\hline September-09 & 0.23 & 0.16 & 0.68 & 0.92 & -0.49 & 1.50 \\
\hline October-09 & 0.23 & 0.12 & 0.63 & 0.88 & -0.46 & 1.40 \\
\hline November-09 & 0.24 & 0.12 & 0.69 & 1.01 & -0.48 & 1.58 \\
\hline
\end{tabular}




\begin{tabular}{|l|c|c|c|c|c|c|} 
December-09 & 0.27 & 0.15 & 0.67 & 1.19 & -0.46 & 1.82 \\
\hline January-10 & 0.28 & 0.19 & 0.69 & 1.20 & -0.46 & 1.90 \\
\hline February-10 & 0.35 & 0.19 & 0.92 & 1.12 & -0.43 & 2.15 \\
\hline March-10 & 0.32 & 0.22 & 0.96 & 1.05 & -0.41 & 2.14 \\
\hline April-10 & 0.38 & 0.26 & 0.99 & 0.99 & -0.41 & 2.21 \\
\hline May-10 & 0.30 & 0.12 & 0.93 & 0.87 & -0.34 & 1.88 \\
\hline June-10 & 0.31 & 0.08 & 0.96 & 0.85 & -0.37 & 1.82 \\
\hline July-10 & 0.29 & -0.04 & 1.00 & 0.80 & -0.34 & 1.70 \\
\hline August-10 & 0.09 & -0.11 & 1.26 & 0.68 & -0.31 & 1.61 \\
\hline
\end{tabular}

Notes: The decomposition is based on an extension of the standard Blinder-Oaxaca decomposition framework, which follows equation 7. Total change in poverty due to wife's working can be factored into five different components (i) change in effect of wife's work for families with unemployed husbands, (ii) change in effect of wife's work for families with NILF husbands, (iii) change in effect of wife's work for families with for employed husbands, (iv) change in proportion of unemployed husbands, and (v) change in proportion of NILF husbands.

The poverty decomposition shows that the total effect of wife's earnings is greater in the later months of 2010 than the beginning months of 2008. Generally, in terms of family poverty reduction, wife's earnings are contributing more at the end than in the beginning months in our observation window. Among the five components in the poverty decomposition, the change in the proportion of unemployed husbands has the greatest role in altering the effect of wife's earnings on monthly family poverty. The decomposition values are negative for the proportion of husbands who are not in the labor force, which implies that effects of a wife working on the monthly poverty levels are reduced as a result of the decline in the proportion of men not in the labor force relative to the beginning month, May 2008.

In contrast to the OPM, poverty decomposition based on the SPM shows a smaller increase in the contribution of wife's earnings (table S1 of appendix C). As we pointed out earlier, government in-kind transfer programs may contribute to poverty reduction in families under the SPM, with the result that wife's earnings contribute less. Using the SPM, we found a similar contribution of wife's earnings due to the change in proportion 
of unemployed husbands. The decomposition using both OPM and SPM suggests that the wife's earnings are particularly important when her husband is unemployed. The decomposition results show that the decline in the proportion of husbands not in the labor force reduces the importance of wife's earnings.

I conducted robustness checks with two additional specifications of the standard decomposition from a variation of the Oaxaca-Blinder decomposition. In particular, I considered an alternative SPM where I omitted taxes, and I considered a version where I omitted SNAP payments. The signs and approximate magnitudes of our main results are not altered by these changes. Estimates are robust to numerous sensitivity analyses and details can be found in appendix D.

\section{Conclusion and Policy Implications}

This study focuses on households that are at the bottom of the income distribution. In this study, I use sampling weights to produce a nationally representative sample from the SIPP survey during the great recession. The SIPP oversamples households with low income, which provides improved precision in results relating to the low income groups. In particular, our analyses of poverty are aided by this oversampling, and this improves our estimates of response to government policy.

I found that wife's earnings make an important contribution to their families' welfare during the great recession. On average, wives' earnings move about ten percent of families from below to above the poverty line. I also extend the investigation by using the government's supplemental poverty measure. The SPM has become increasingly important due to the growth of government transfer programs and increase in taxes. 
Although we observe slightly different levels of absolute poverty for the official and the supplemental measures, the observed patterns, and especially the role of women's labor force participation, was not influenced by which measure we use. This suggests that household poverty is not very responsive to the differences in these measures, which result from disparate treatment of certain government transfers and income tax liabilities. Alternatively, the transfers and liabilities may largely balance out, so the difference has little impact.

I extended the analysis using a decomposition that distinguished the effects of women's labor force participation into five categories. In particular, households are distinguished based on the labor force status of their husbands: employed, unemployed, and not in the labor force. The decomposition analysis focuses on how wives' labor force participation affects poverty for each of these kinds of families as well as the role of changes in the three proportions. We found that the proportion of unemployed husbands has the greatest influence on the overall effect. Again, the finding remains the same if we use the supplemental poverty measure.

These results underscore the continued importance of wives' earnings in preventing poor families from falling below the poverty line. Policies that facilitate wives' participation in the labor force provide clear benefits for poor families, especially during economic downturns.

This study's findings have important implications for the economic environment of the post-COVID-19 pandemic era. Unlike many previous recessions, the pandemic has caused more considerable employment losses for women than for men and is known as "shecession" (she-recession). The relatively stable sectors of education, healthcare, and 
other parts of service sectors disproportionately employ women. These industries are hit hard by the coronavirus. Moreover, women's labor supply has declined substantially during these coronavirus times relative to men's labor supply. In many families where both husband and wife worked, the pandemic forced mothers, not fathers, to quit a job due to children's virtual schooling and other factors. This suggests that wives may no longer be serving as insurance for the families when husbands experience unemployment. Given our approach, we would expect that women's labor force effect on poverty could well be smaller during the COVID-19 pandemic than what we found in this study, in large part reflecting the lower participation rate of women. 


\section{References}

Agrawal, T., \& Desai, S. (2017). The Impact of Women's Earnings on Household Income Inequality in India. PAA 2017 Annual Meeting, Chicago.

Betson, D., \& Van Der Gaag, J. (1984). Working married women and the distribution of income. Journal of Human Resources, 19(4), 532-543.

Bishop, J. A., Lee, J., \& Zeager, L. A. (2018). Adjusting for family size in the supplemental poverty measure. Applied Economics Letters, 25(8), 553-556.

Blank, R. M., \& Blinder, A. S. (1985). Macroeconomics, income distribution, and poverty (No. 0898-2937). National Bureau of Economic Research.

Blau, F.D., \& Winkler, A. E. (2017). The Economics of Women, Men, and Work. Oxford University Press.

Blau, Francine D. (1998). Trends in the Well-Being of American Women, 1970-1995. Journal of Economic Literature, 36(1), 112-165. JSTOR. www.jstor.org/stable/2564953

Blau, Francine D, \& Kahn, L. M. (2007). Changes in the labor supply behavior of married women: 1980-2000. Journal of Labor Economics, 25(3), 393-438.

Blinder, A. S. (1973). Wage discrimination: Reduced form and structural estimates. Journal of Human Resources, 8(4), 436-455.

Cancian, M., \& Reed, D. (1998). Assessing the effects of wives' earnings on family income inequality. Review of Economics and Statistics, 80(1), 73-79.

Cancian, M., \& Reed, D. (1999). The impact of wives' earnings on income inequality: Issues and estimates. Demography, 36(2), 173-184. 
Cancian, M., \& Reed, D. (2008). Family structure, childbearing, and parental employment: Implications for the level and trend in poverty. University of Wisconsin-Madison, Institute for Research on Poverty.

Cattan, P. (1998). The effect of working wives on the incidence of poverty. Monthly Lab. Rev., 121, 22.

Cullen, J. B., \& Gruber, J. (2000). Does unemployment insurance crowd out spousal labor supply? Journal of Labor Economics, 18(3), 546-572.

Czajka, J. L., Mabli, J., \& Cody, S. (2008). Sample loss and survey bias in estimates of Social Security beneficiaries: A tale of two surveys. Washington, $D C$ : Mathematica Policy Research, Inc.

Dalaker, J. (2001). Poverty in the United States, 2000. US Department of Commerce, Census Bureau.

Danziger, S., \& Gottschalk, P. (1995). America unequal. Harvard University Press.

Devine, J. A., \& Wright, J. D. (1993). The greatest of evils: Urban poverty and the American underclass. Transaction Publishers.

Feenberg, D., \& Coutts, E. (1993). An introduction to the TAXSIM model. Journal of Policy Analysis and Management, 12(1), 189-194.

Fernandes, R., \& Felício, F. de. (2005). The entry of the wife into the labor force in response to the husband's unemployment: A study of the added worker effect in Brazilian Metropolitan areas. Economic Development and Cultural Change, 53(4), 887-911.

Fisher, G. M. (1992). Poverty Guidelines for 1992. Social Security Bulletin, 55(1), 43. 
Fox, L. (2018). The supplemental poverty measure: 2017: Current population reports. US Census Bureau, Washington, DC.

Garcia-Escribano, M. M. (2004). Does spousal labor smooth fluctuations in husbands' earnings? The role of liquidity constraints (No. 4-20). International Monetary Fund.

Gong, X. (2011). The added worker effect for married women in Australia. Economic Record, 87(278), 414-426.

Hardoy, I., \& Schøne, P. (2014). Displacement and household adaptation: Insured by the spouse or the state? Journal of Population Economics, 27(3), 683-703.

Haveman, R., \& Schwabish, J. (1999). Economic growth and poverty: A return to normalcy? Focus, 20(2), 1-7.

Iceland, J. (2003a). Why poverty remains high: The role of income growth, economic inequality, and changes in family structure, 1949-1999. Demography, 40(3), 499519.

Iceland, J. (2003b). Why poverty remains high: The role of income growth, economic inequality, and changes in family structure, 1949-1999. Demography, 40(3), 499519.

Jones Jr, A. F., \& Weinberg, D. H. (2000). The changing shape of the nation's income distribution. Current Population Reports, 60, 1-11.

Karaoglan, D., \& Okten, C. (2015). Labor-Force Participation of Married Women in Turkey: A Study of the Added-Worker Effect and the Discouraged-Worker Effect. Emerging Markets Finance and Trade, 51(1), 274-290. 
Kimberlin, S., Shaefer, H. L., \& Kim, J. (2016). Measuring poverty using the supplemental poverty measure in the panel study of income dynamics, 1998 to 2010. Journal of Economic and Social Measurement, 41(1), 17-47.

Mankart, J., \& Oikonomou, R. (2016). The rise of the added worker effect. Economics Letters, 143, 48-51.

Mattingly, M. J., \& Smith, K. E. (2010). Changes in Wives' Employment When Husbands Stop Working: A Recession-Prosperity Comparison. Family Relations, 59(4), 343-357.

Meyer, B. D., \& Sullivan, J. X. (2012). Identifying the disadvantaged: Official poverty, consumption poverty, and the new supplemental poverty measure. Journal of Economic Perspectives, 26(3), 111-136.

Oaxaca, R. (1973). Male-female wage differentials in urban labor markets. International Economic Review, 14(3), 693-709.

Parker, S. W., \& Skoufias, E. (2004). The added worker effect over the business cycle: Evidence from urban Mexico. Applied Economics Letters, 11(10), 625-630.

Pencavel, J. (2007). Earnings inequality, labour supply and schooling in husband-wife families. Bulletin of Economic Research, 59(2), 83-124.

Short, K., \& Renwick, T. (2010). Supplemental poverty measure: Preliminary estimates for 2008. 32nd Annual Research Conference of the Association on for Public Policy Analysis and Management, Boston, MA.

Sudo, N. (2017). The effects of women's labor force participation: An explanation of changes in household income inequality. Social Forces, 95(4), 1427-1450. 
Thompson, J. P., \& Smeeding, T. M. (2013). Inequality and poverty in the United States: The aftermath of the Great Recession. Federal Reserve Board, Washington, D.C. U.S. Census Bureau. (n.d.). 2008 Panel Wave 01. The United States Census Bureau. Retrieved October 26, 2020, from https://www.census.gov/programssurveys/sipp/data/datasets/2008-panel/wave-1.html 


\section{Appendix}

\section{$\underline{\text { Appendix A }}$}

\section{Estimation of the supplemental poverty measure}

The supplemental poverty measure (SPM) differs from the official measure because of the way it treats government transfers and income tax. There are five main transfer payments counted in the SPM but in not the official measure. The SIPP survey allows us to account for three of them. First, the Supplemental Nutrition Assistance Program (SNAP) (previously food stamps), is received each month in the form of an electronic benefit transfer card, which can be used to buy food. In the survey data for the sample period, there is a maximum amount of SNAP benefit in our sample of $\$ 2,840$ for a family in a month. Second are school meals for children, which includes school breakfast and school lunch. In our sample, $15.5 \%$ of households in a month receive school breakfast, and $36 \%$ of the family months in the sample receive school lunch. The total benefit of school meal per family per month is defined by the average cost of a school lunch incurred by the program and the number of children receiving the meal. Third, the Supplementary Nutrition Program for Women Infants and Children (WIC) benefits are reported in dollars for a month with a maximum payment of \$219 in the sample.

There are two other types of government transfers reported in the SIPP survey, and I am unable to account for these two types of payments. First, although SIPP reports whether a family receives housing subsidies, no dollar amount was reported in the SIPP survey. In less than 1 percent of the family months was it reported that the household received such subsidies. I excluded the housing subsidy information due to the low 
representativeness of data and the binary response of the survey questionnaire. Second, whether a family received payments from the Low-Income Home Energy Assistance Program is also reported. Less than 1 percent of the sample said that they received such support, and hence I have excluded this program from our analysis.

The supplemental poverty measure subtracts five items from the income measure that are not considered in the official measure: (i) taxes, (ii) expenses related to work, (iii) child care expenses, (iv) medical out-of-pocket expenses (MOOP), and (v) child support paid. None these items are surveyed in the SIPP. However, we are able to adjust for some taxes. Our analysis calculated the federal income tax, state income tax, and federal payroll tax information using the TAXSIM program from NBER. TAXSIM is a microsimulation model used to calculate annual U.S. federal and state taxes (Feenberg \& Coutts, 1993; Kimberlin et al., 2016). ${ }^{16}$

\section{Simulation using TAXSIM}

In the TAXSIM simulation, there are 27 different fields to run the simulation in order to calculate tax liabilities. TAXSIM requires some demographic and socioeconomic variables as well, including tax assessment year, state name, marital status, age of primary taxpayer, and age of spouse. TAXSIM also requires dependents information. I also set the number of qualifying children under 19 for Earned Income Tax Credit to zero due to lack of information. TAXSIM has 17 fields related to earnings and/or income. This includes wage and salary income, and self-employment income of the primary taxpayer, and wage and salary income, and self-employment income of the spouse. Both of these earnings measures were available in the SIPP on a monthly basis, and I converted these to

\footnotetext{
${ }^{16} \mathrm{TAXSIM}$ can be accessed at the NBER website http://users.nber.org/ $\operatorname{taxsim} / \operatorname{taxsim} 27 /$.
} 
an annual basis (multiplying by 12) for TAXSIM. I have annual dividend income and interest received. I set short term capital gains or losses and long term capital gains or losses to zero due to lack of information. I have other property income, but I don't have other non-property income. For taxable pensions and I have added six types of pensions listed in the SIPP data and I subtract IRA distributions. Gross social security benefits are from the adult Social Security and child Social Security. I have unemployment compensation received. I use other non-taxable transfer income, which includes total means-tested cash transfers for the reference month, which we convert to an annual basis. Amount of monthly rent data also is converted to an annual basis (multiplying by 12). I don't have real estate taxes paid, other itemized deductions, child care expenses and we set these to zero. I have mortgage deductions defined by the amount of interest paid on own mortgage.

The TAXSIM requires annual data to run the simulation. On the other hand, our analysis requires monthly data since other SIPP survey data are monthly. Monthly taxes were constructed from the annual tax bill under the assumption that all months would have the same figures as the current month and are then allocated to the month by dividing by 12 .

\section{$\underline{\text { Appendix B }}$}

Figure S1 shows the percentage of families below poverty each month based on the supplemental poverty measure (SPM) rather than the official measure (OPM). We see a 32.9 percentage points increase of families below poverty from first to last month in the sample (May 2008 to August 2010), an increase from 7.1 percent to 9.5 percent of our sample families. On average, 7.8 percent of families were below poverty during the 
sample period. When I subtract wives' earnings from family income, the SPM implies that an average of 17.0 percent of families would be below poverty over the full sample period.

Figure S1. Distribution of poverty by month: percent of family in poverty based on supplemental poverty measure

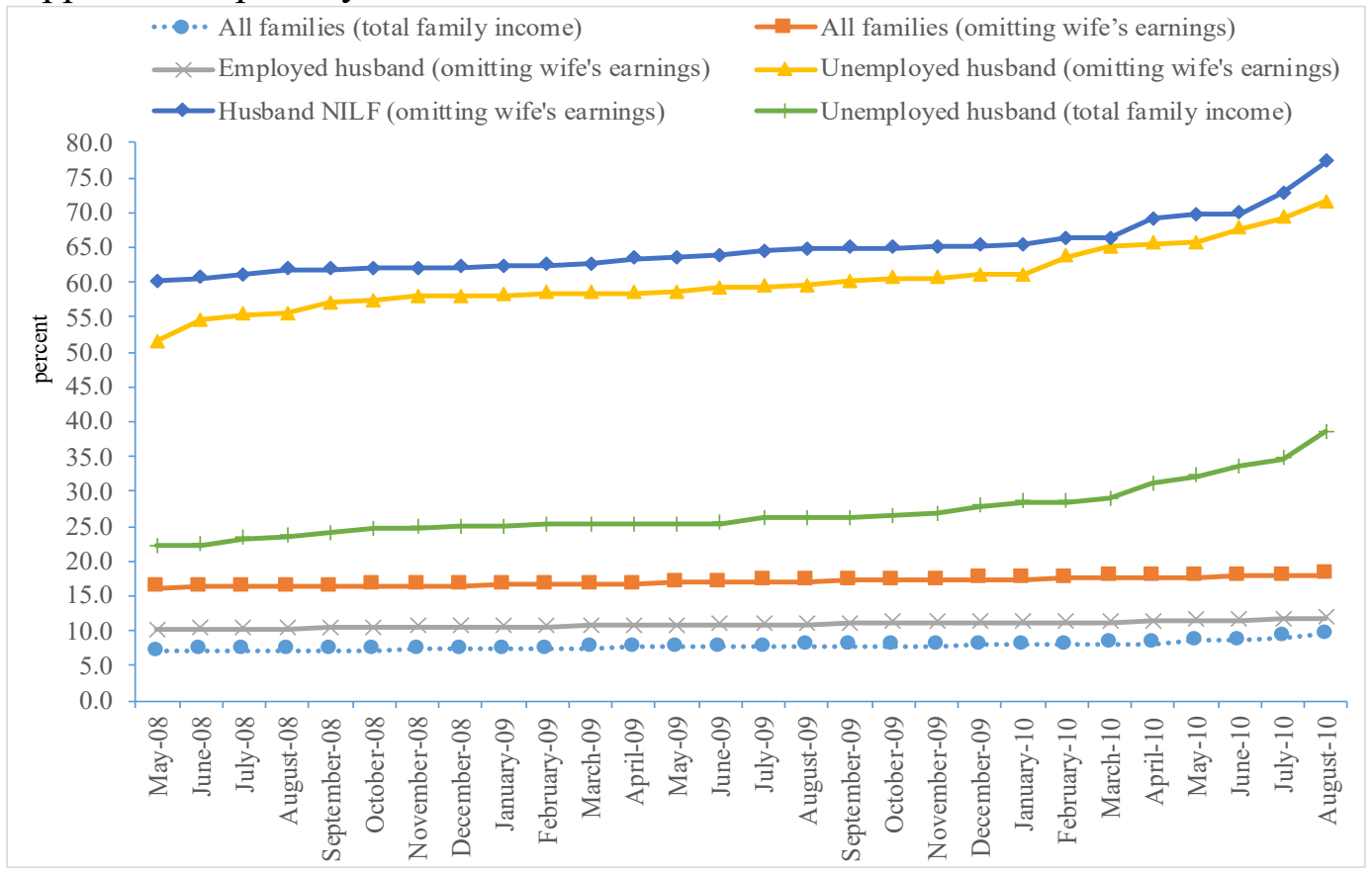

Source. Data analysis from wave 1 to wave 7 of SIPP 2008.

When we move to the SPM, in figure S1, after omitting wife's earnings, the number of families below poverty is marginally lower than for the OPM for each of the three kinds of families based on employment status of husbands in figure 1 in the main text. Without wife's earnings, on average families with employed husbands, unemployed husbands and husbands not in the labor force have poverty rates of 10.9 percent, 60.3 percent, and 64.8 percent, respectively, based on the SPM, as opposed to 12.4 percent, 62.6 percent, and 67.2 percent based on OPM. The proportion of families below poverty is higher in the OPM relative to the SPM in our sample window. 
In figure S2, wives' earnings are defined as the income generated by a job or selfemployment. Income from investment and government transfers are not included in wives' earnings. Both the extensive and intensive margins contribute to this trend. Some women lost their jobs due to the recession, and some worked fewer hours.

Figure S2. Employed wives' mean earned income

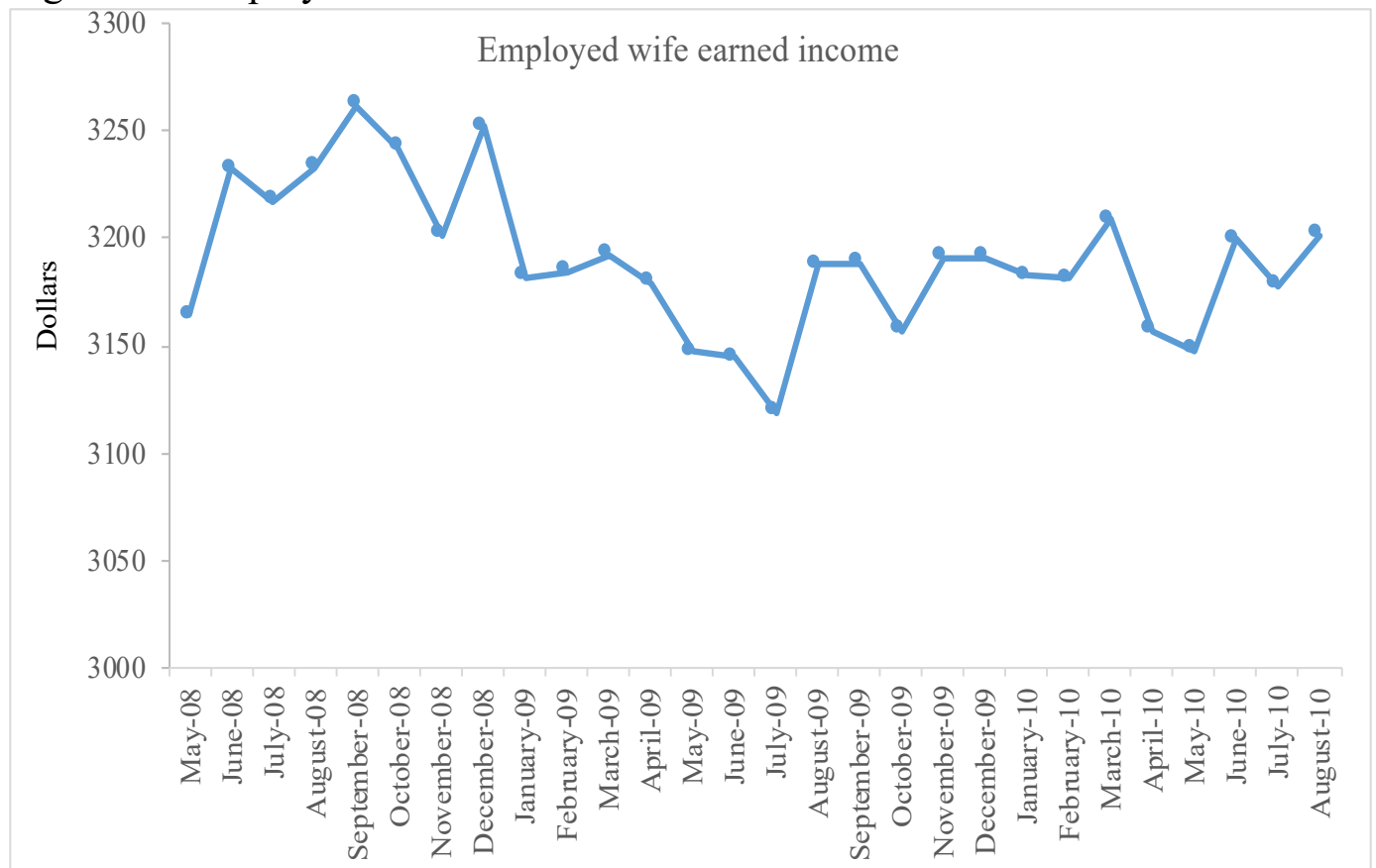

Source. Data analysis from wave 1 to wave 7 of SIPP 2008.

In figure S3, we see that the average Unemployment Insurance benefit payment (contingent on receiving any benefit) for husbands increased gradually through the first half of our period. Over our period, the average monthly benefit amount was $\$ 1,187$, contingent on receiving any payment during the month. The number of Unemployment Insurance claims have increased during the recession, in part reflecting the fact that benefits were extended beyond the regular UI period in most states. The second line (circled marker) in the figure provides the average of the sum of husbands' and wives' UI 
benefit payments for households receiving both. The joint unemployment insurance benefits increased dramatically in the first six months and then decreased.

Figure S3. Mean unemployment insurance benefits

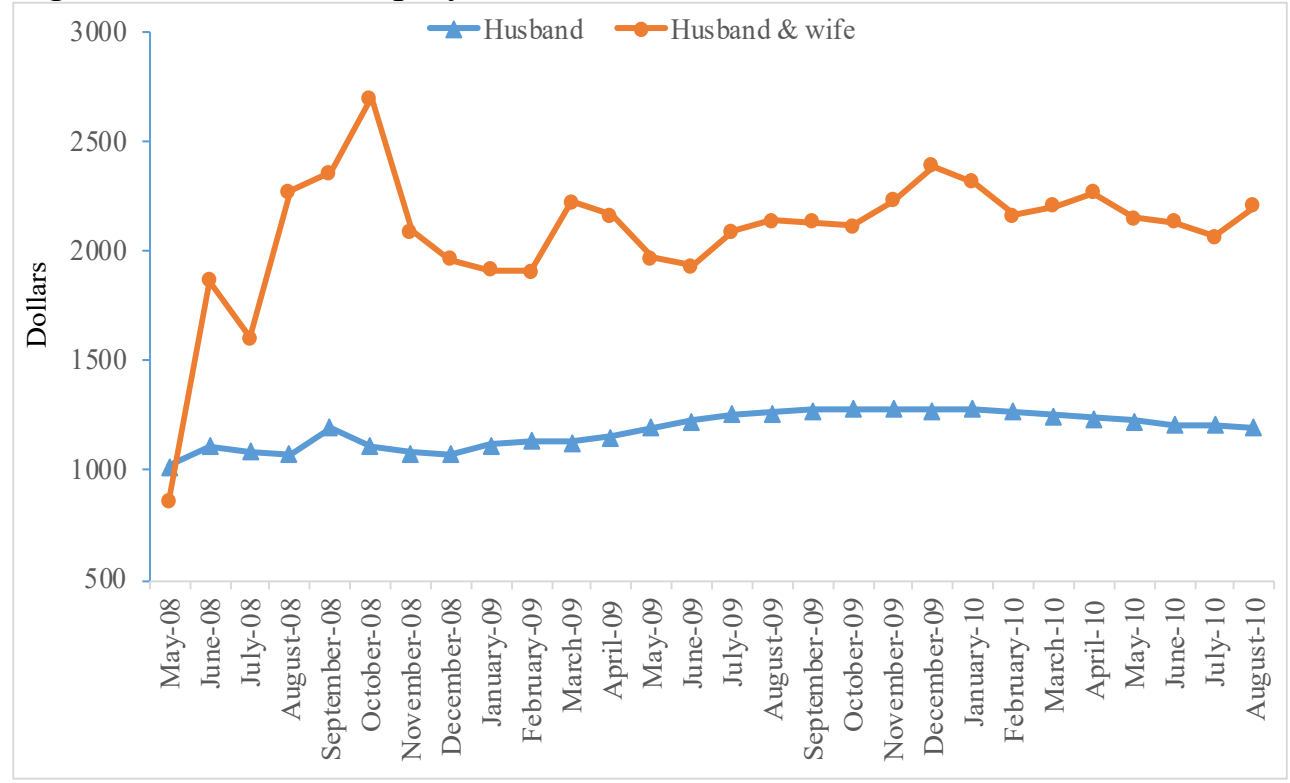

Source. Data analysis from wave 1 to wave 7 of SIPP 2008.

Figure S4. Poverty and family income by state (2008-2010)

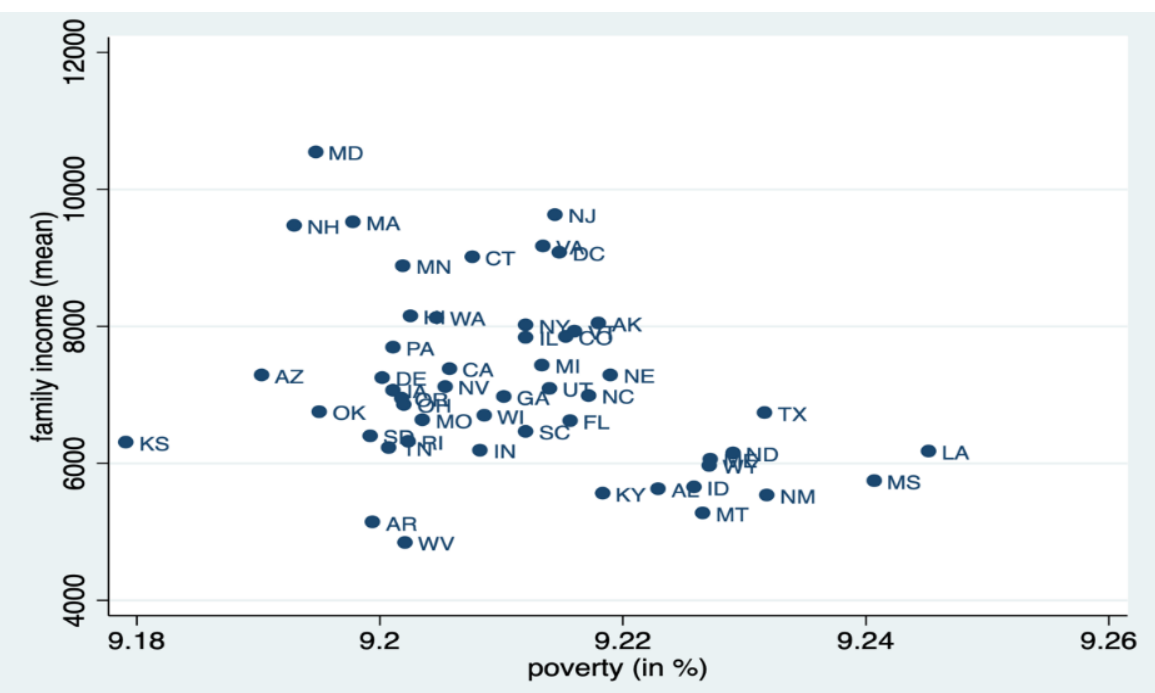

Source. Data analysis from wave 1 to wave 7 of SIPP 2008. 
Figure S4 presents state-level poverty and income data, we see states like Maryland and New Hampshire had low poverty levels and high average family income. Similarly, other states had high poverty and low average family income. States like Louisiana and Mississippi were in this category. Perhaps most notable, differences across states in poverty for the families in our sample were quite modest, varying from $9.18 \%$ to $9.25 \%$, much smaller than differences in average income. This suggests that the lower end of the income distribution for married couples did not differ dramatically among states even when the mean of the overall distribution varied dramatically.

In figure S5, based on SPM, the average effect of the wife's earnings on family poverty across all months is 9.2 percentage points, as defined by equation 1 in the main text. This tells us that the wife's monetary contribution has important implications for family poverty reduction. The effect increases only slightlyas we move from the first months to later months, and then declines, averaging 9.1 percentage points in the last six months of our observation window. Conversely, based on the OPM, the effect was becoming more important as we move to later months of the great recession, averaging 10.0 percentage points in the last six months of our observation window. We show below that the wife's income does not increase in importance during the recession based on the SPM as much as in the OPM because of the greater reliance by families on government transfer programs. In the presence of such benefits, wives' contributions became relatively less important to the families. 
Figure S5. Effect of wife's earnings on the level of poverty based on supplemental poverty measure

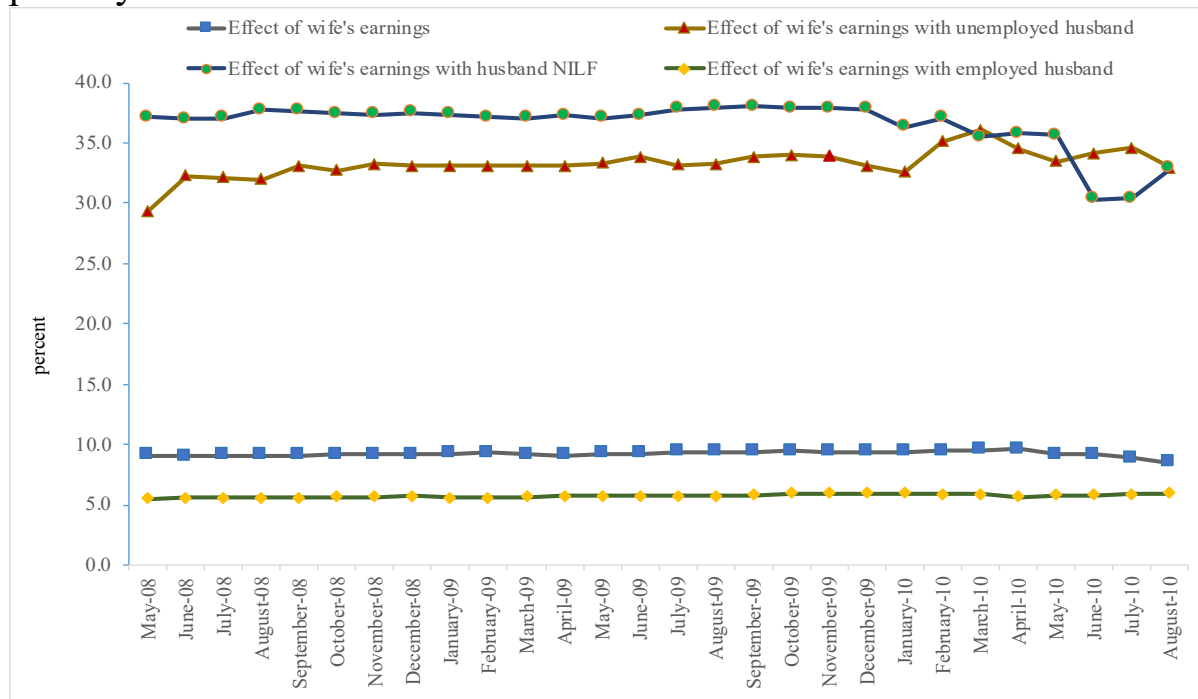

Source. Data analysis from wave 1 to wave 7 of SIPP 2008 and TAXSIM.

We have three other measures of a working wife's effect on the level of poverty depending on the husband's labor force statuses of employed, unemployed and not in the labor force. Both in OPM and SPM, the wife's earnings has the greatest effects when her husband is not in the labor force (35.4 percent and 36.5 percent in OPM and SPM, respectively). When her husband is unemployed the effects are 32.1 percent and 33.3 percent in OPM and SPM, respectively. The effect is smallest when her husband is employed (6.1 percent and 5.7 percent in OPM and SPM). When husbands are not in the labor force, wife's earnings are less important to the family poverty reduction in the last six months relative to first six months of our observation window (33.4 percentage points vs. 37.3 percentage points). 


\section{$\underline{\text { Appendix C }}$}

\section{Poverty Decomposition based on SPM}

Table S1. Poverty decomposition $\left(t^{\prime}<t\right)$ for each month compare to first month ( $t^{\prime}$ : May 2008), Supplemental Poverty Measure (SPM).

\begin{tabular}{|c|c|c|c|c|c|c|}
\hline Month \& Year & $\begin{array}{l}\text { Due to change } \\
\text { for unemployed } \\
\text { husbands }\end{array}$ & $\begin{array}{l}\text { Due to change } \\
\text { for NILF } \\
\text { husbands }\end{array}$ & $\begin{array}{l}\text { Due to } \\
\text { change for } \\
\text { employed } \\
\text { husbands }\end{array}$ & $\begin{array}{l}\text { Due to change } \\
\text { in proportion of } \\
\text { unemployed } \\
\text { husband }\end{array}$ & $\begin{array}{l}\text { Due to } \\
\text { change in } \\
\text { proportion } \\
\text { of NILF } \\
\text { husbands }\end{array}$ & $\begin{array}{l}\text { Total change } \\
\text { in poverty } \\
\text { due to wife's } \\
\text { working }\end{array}$ \\
\hline June- 08 & 0.10 & -0.01 & 0.12 & 0.15 & -0.20 & 0.14 \\
\hline July-08 & 0.10 & -0.01 & 0.07 & 0.27 & -0.34 & 0.09 \\
\hline August-08 & 0.09 & 0.04 & 0.06 & 0.25 & -0.36 & 0.09 \\
\hline September-08 & 0.13 & 0.03 & 0.10 & 0.24 & -0.49 & 0.01 \\
\hline October-08 & 0.13 & 0.02 & 0.14 & 0.38 & -0.61 & 0.06 \\
\hline November- 08 & 0.16 & 0.02 & 0.17 & 0.50 & -0.58 & 0.26 \\
\hline December-08 & 0.18 & 0.02 & 0.19 & 0.89 & -0.54 & 0.74 \\
\hline January-09 & 0.18 & 0.01 & 0.11 & 0.92 & -0.56 & 0.66 \\
\hline February-09 & 0.18 & 0.00 & 0.08 & 0.93 & -0.55 & 0.65 \\
\hline March-09 & 0.18 & 0.00 & 0.16 & 0.95 & -0.52 & 0.77 \\
\hline April-09 & 0.19 & 0.01 & 0.20 & 0.98 & -0.59 & 0.78 \\
\hline May-09 & 0.19 & 0.00 & 0.21 & 0.91 & -0.54 & 0.76 \\
\hline June-09 & 0.22 & 0.01 & 0.20 & 1.00 & -0.56 & 0.87 \\
\hline July-09 & 0.19 & 0.04 & 0.19 & 1.06 & -0.58 & 0.91 \\
\hline August-09 & 0.20 & 0.05 & 0.22 & 1.05 & -0.58 & 0.94 \\
\hline September-09 & 0.22 & 0.05 & 0.27 & 0.99 & -0.54 & 0.99 \\
\hline October-09 & 0.23 & 0.05 & 0.39 & 0.94 & -0.51 & 1.11 \\
\hline November-09 & 0.24 & 0.05 & 0.39 & 1.09 & -0.53 & 1.23 \\
\hline December-09 & 0.21 & 0.04 & 0.38 & 1.25 & -0.50 & 1.39 \\
\hline January-10 & 0.18 & -0.05 & 0.39 & 1.24 & -0.49 & 1.27 \\
\hline February-10 & 0.30 & 0.00 & 0.38 & 1.19 & -0.46 & 1.41 \\
\hline March-10 & 0.35 & -0.10 & 0.38 & 1.15 & -0.43 & 1.34 \\
\hline April-10 & 0.26 & -0.08 & 0.14 & 1.03 & -0.43 & 0.91 \\
\hline May-10 & 0.20 & -0.10 & 0.25 & 0.91 & -0.37 & 0.89 \\
\hline June-10 & 0.23 & -0.44 & 0.30 & 0.89 & -0.37 & 0.61 \\
\hline July-10 & 0.24 & -0.44 & 0.32 & 0.85 & -0.35 & 0.62 \\
\hline August-10 & 0.16 & -0.28 & 0.42 & 0.77 & -0.34 & 0.74 \\
\hline
\end{tabular}


Table S2. Poverty decomposition $\left(t^{\prime}<t\right)$ for each month compare to first month $\left(t^{\prime}\right.$ : May 2008), Supplemental Poverty Measure (SPM), with family income excluding SNAP.

\begin{tabular}{|c|c|c|c|c|c|c|}
\hline Month \& Year & $\begin{array}{l}\text { Due to } \\
\text { change for } \\
\text { unemployed } \\
\text { husbands }\end{array}$ & $\begin{array}{l}\text { Due to } \\
\text { change for } \\
\text { NILF } \\
\text { husbands }\end{array}$ & $\begin{array}{l}\text { Due to } \\
\text { change for } \\
\text { employed } \\
\text { husbands }\end{array}$ & $\begin{array}{l}\text { Due to change } \\
\text { in proportion of } \\
\text { unemployed } \\
\text { husband }\end{array}$ & $\begin{array}{l}\text { Due to change } \\
\text { in proportion } \\
\text { of NILF } \\
\text { husbands }\end{array}$ & $\begin{array}{c}\text { Total change } \\
\text { in poverty } \\
\text { due to wife's } \\
\text { working }\end{array}$ \\
\hline June-08 & 0.11 & 0.08 & 0.09 & 0.14 & -0.19 & 0.23 \\
\hline July-08 & 0.10 & 0.08 & -0.11 & 0.26 & $\begin{array}{l}-0.32 \\
\end{array}$ & 0.02 \\
\hline August-08 & 0.08 & 0.05 & -0.07 & 0.24 & -0.33 & -0.02 \\
\hline September-08 & 0.11 & 0.06 & -0.03 & 0.23 & -0.45 & -0.08 \\
\hline October-08 & 0.15 & 0.07 & -0.06 & 0.37 & -0.57 & -0.04 \\
\hline November-08 & 0.15 & 0.10 & -0.04 & 0.48 & -0.54 & 0.15 \\
\hline December-08 & 0.20 & 0.09 & -0.02 & 0.87 & -0.50 & 0.63 \\
\hline January-09 & 0.23 & 0.09 & $\begin{array}{c}-0.01 \\
\end{array}$ & 0.91 & $\begin{array}{c}-0.53 \\
\end{array}$ & 0.70 \\
\hline February-09 & 0.23 & 0.11 & -0.07 & 0.92 & -0.51 & 0.68 \\
\hline March-09 & 0.22 & 0.11 & -0.08 & 0.94 & -0.49 & 0.70 \\
\hline April-09 & 0.22 & 0.12 & -0.10 & 0.96 & -0.56 & 0.64 \\
\hline May-09 & 0.20 & 0.13 & $\begin{array}{l}-0.04 \\
\end{array}$ & 0.88 & -0.51 & 0.66 \\
\hline June-09 & 0.21 & 0.12 & 0.12 & 0.96 & -0.53 & 0.88 \\
\hline July-09 & 0.22 & 0.12 & 0.13 & 1.03 & -0.54 & 0.96 \\
\hline August-09 & 0.21 & 0.13 & 0.12 & 1.02 & -0.54 & 0.94 \\
\hline September-09 & 0.21 & 0.12 & 0.11 & 0.95 & -0.50 & 0.89 \\
\hline October-09 & 0.23 & 0.12 & 0.23 & 0.92 & $\begin{array}{l}-0.47 \\
\end{array}$ & 1.02 \\
\hline November-09 & 0.23 & 0.10 & 0.23 & 1.05 & -0.49 & 1.12 \\
\hline December-09 & 0.19 & 0.10 & 0.23 & 1.21 & -0.46 & 1.26 \\
\hline January-10 & 0.20 & 0.13 & 0.23 & 1.21 & -0.47 & 1.31 \\
\hline February-10 & 0.28 & 0.11 & 0.27 & 1.14 & -0.43 & 1.38 \\
\hline March-10 & 0.28 & 0.15 & 0.27 & 1.08 & -0.42 & 1.35 \\
\hline April-10 & 0.30 & 0.20 & 0.27 & 1.01 & -0.42 & 1.37 \\
\hline May-10 & 0.30 & 0.10 & 0.25 & 0.91 & -0.35 & 1.20 \\
\hline June-10 & 0.26 & 0.09 & 0.31 & 0.87 & -0.39 & 1.14 \\
\hline July-10 & 0.29 & -0.10 & 0.39 & 0.84 & -0.35 & 1.07 \\
\hline August-10 & 0.15 & -0.14 & 0.41 & 0.73 & -0.32 & 0.83 \\
\hline
\end{tabular}


Table S3. Poverty decomposition $\left(t^{\prime}<t\right)$ for each month compare to first month $\left(t^{\prime}\right.$ : May 2008), Supplemental Poverty Measure (SPM), with family income not excluding income tax.

\begin{tabular}{|c|c|c|c|c|c|c|}
\hline Month \& Year & $\begin{array}{l}\text { Due to } \\
\text { change for } \\
\text { unemploye } \\
\text { d husbands }\end{array}$ & $\begin{array}{l}\text { Due to } \\
\text { change for } \\
\text { NILF } \\
\text { husbands }\end{array}$ & $\begin{array}{l}\text { Due to } \\
\text { change for } \\
\text { employed } \\
\text { husbands }\end{array}$ & $\begin{array}{l}\text { Due to } \\
\text { change in } \\
\text { proportion of } \\
\text { unemployed } \\
\text { husband }\end{array}$ & $\begin{array}{l}\text { Due to } \\
\text { change in } \\
\text { proportion of } \\
\text { NILF } \\
\text { husbands }\end{array}$ & $\begin{array}{l}\text { Total change } \\
\text { in poverty } \\
\text { due to wife's } \\
\text { working }\end{array}$ \\
\hline June- 08 & 0.07 & 0.00 & 0.27 & 0.14 & -0.19 & 0.28 \\
\hline July-08 & 0.12 & -0.01 & 0.23 & 0.26 & -0.32 & 0.28 \\
\hline August- 08 & 0.11 & 0.03 & 0.13 & 0.25 & -0.34 & 0.19 \\
\hline September-08 & 0.14 & 0.03 & 0.11 & 0.24 & -0.46 & 0.05 \\
\hline October- 08 & 0.13 & 0.04 & 0.20 & 0.37 & -0.58 & 0.15 \\
\hline November-08 & 0.17 & 0.04 & 0.22 & 0.48 & -0.55 & 0.36 \\
\hline December-08 & 0.20 & 0.03 & 0.21 & 0.87 & -0.51 & 0.81 \\
\hline January-09 & 0.19 & 0.03 & 0.20 & 0.90 & -0.53 & 0.78 \\
\hline February- 09 & 0.18 & 0.03 & 0.23 & 0.90 & -0.52 & 0.83 \\
\hline March-09 & 0.17 & 0.04 & 0.26 & 0.92 & -0.50 & 0.89 \\
\hline April-09 & 0.17 & 0.07 & 0.28 & 0.95 & -0.57 & 0.90 \\
\hline May-09 & 0.15 & 0.06 & 0.29 & 0.86 & -0.52 & 0.85 \\
\hline June-09 & 0.15 & 0.07 & 0.27 & 0.94 & -0.54 & 0.88 \\
\hline July-09 & 0.16 & 0.09 & 0.28 & 1.02 & -0.56 & 0.99 \\
\hline August-09 & 0.17 & 0.11 & 0.40 & 1.01 & -0.56 & 1.13 \\
\hline September-09 & 0.20 & 0.10 & 0.40 & 0.95 & -0.52 & 1.13 \\
\hline October-09 & 0.20 & 0.10 & 0.39 & 0.91 & -0.48 & 1.11 \\
\hline November-09 & 0.20 & 0.09 & 0.43 & 1.05 & -0.51 & 1.26 \\
\hline December-09 & 0.25 & 0.06 & 0.49 & 1.23 & -0.47 & 1.56 \\
\hline January-10 & 0.24 & 0.06 & 0.45 & 1.24 & -0.48 & 1.51 \\
\hline February-10 & 0.32 & -0.01 & 0.50 & 1.16 & -0.43 & 1.55 \\
\hline March-10 & 0.36 & -0.18 & 0.55 & 1.12 & -0.40 & 1.45 \\
\hline April-10 & 0.29 & -0.06 & 0.67 & 1.00 & -0.40 & 1.50 \\
\hline May-10 & 0.24 & -0.33 & 0.70 & 0.89 & -0.32 & 1.17 \\
\hline June-10 & 0.24 & -0.66 & 0.68 & 0.87 & -0.32 & 0.81 \\
\hline July-10 & 0.28 & -0.47 & 0.69 & 0.83 & -0.33 & 1.01 \\
\hline August-10 & 0.19 & -0.18 & 0.93 & 0.74 & -0.32 & 1.36 \\
\hline
\end{tabular}




\section{$\underline{\text { Appendix D }}$}

\section{Sensitivity Analyses}

Analyses based on the supplemental poverty measure (SPM) show the effects of the wife's earnings are slightly lower than analyses based on the official poverty measure (OPM). The inclusion in income of government in-kind transfers, and exclusion of income tax liability make the SPM different from the OPM. In this section, I want to see how sensitive estimates of the effects of wife's earnings on poverty are to SNAP payments. Among the in-kind government transfer programs examined in this paper, SNAP plays an important role. In our sample, only 6.4 percent of family-months receive SNAP payments, but it plays an important role for those near poverty. Families that receive SNAP have an average monthly SNAP payment of \$412. Figure S6 shows the distribution of poverty by month where I consider the level of poverty based on the supplemental poverty measure but omit SNAP benefits from family income. Figure 6 is comparable to figure S1 (section 2) which includes SNAP.

In the sample period, the level of poverty based on the supplemental poverty measure increases by about 1 percentage point if I omit SNAP from income. On average, 8.7 percent of families are below poverty after omitting SNAP, in contrast to 7.8 percent of families who were below poverty when we do not omit SNAP (figure S1). In the analyses omitting SNAP, when we omit wife's earnings, 17.9 percent of families would be in poverty, compared to 17.0 percent of families in poverty when wife's earnings are included. If I consider families with unemployed husbands and omit wife's earnings, 62.0 percent of families are in poverty when I omit SNAP, as opposed to 60.4 percent of families when SNAP is included. 
Figure S6. Distribution of poverty by month: percent of family in poverty based on supplemental poverty measure (omitting SNAP)

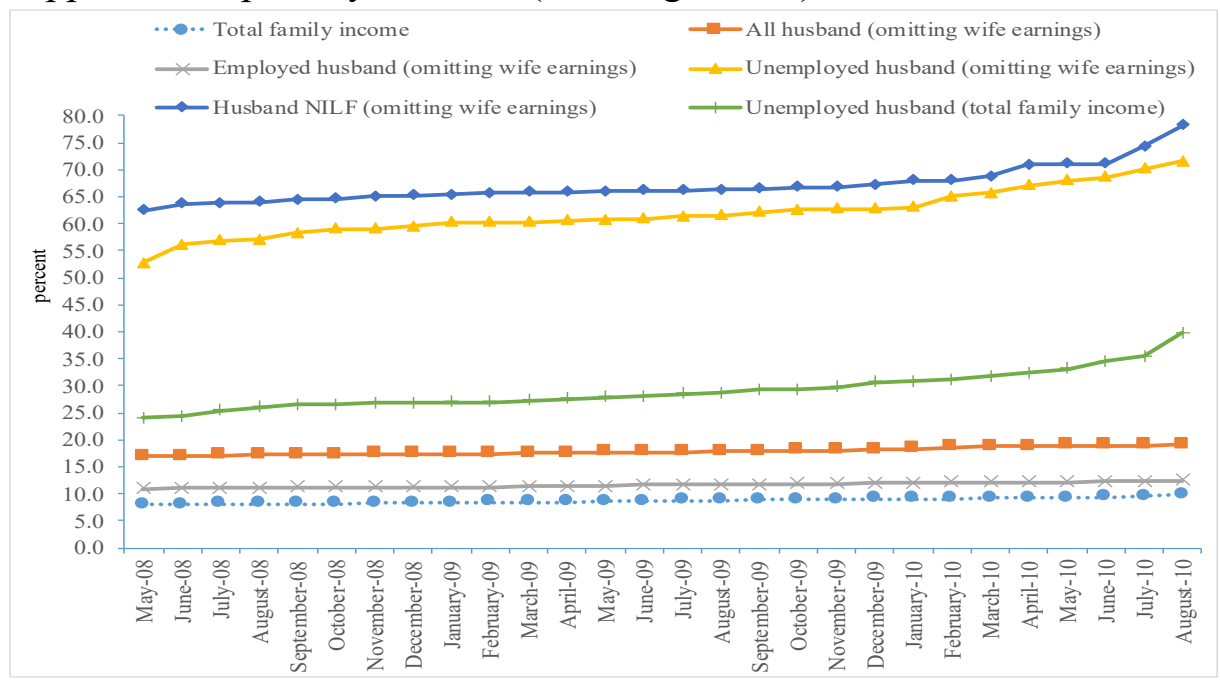

Source. Data analysis from wave 1 to wave 7 of SIPP 2008 and TAXSIM.

The effect of wife's earnings, omitting SNAP, on the level of poverty based on the supplemental poverty measure is shown by the figure S7. This is comparable to figure S5 (section 2) where I did not omit SNAP benefits when measuring the poverty based on supplemental poverty measure. After omitting SNAP, the effect of wife's earnings on the level of poverty averages 9.2 percentage points, which remains the same when I include SNAP, i.e., wife's earnings reduce the proportion of families in poverty by 9.2 percentage points. When I omit SNAP benefits from total family income, the average effect of wife's work for families with unemployed husbands is 32.8 percent, 35.8 percent for families with husbands not in the labor force, and 5.8 percent for families with employed husbands. The decomposition analysis also shows similar result when I analyze the contribution of wife's work with and without SNAP benefits (table S1 \& S2 of section 3). 
Figure S7. Effect of wife's earnings on the level of poverty based on supplemental poverty measure (omitting SNAP)

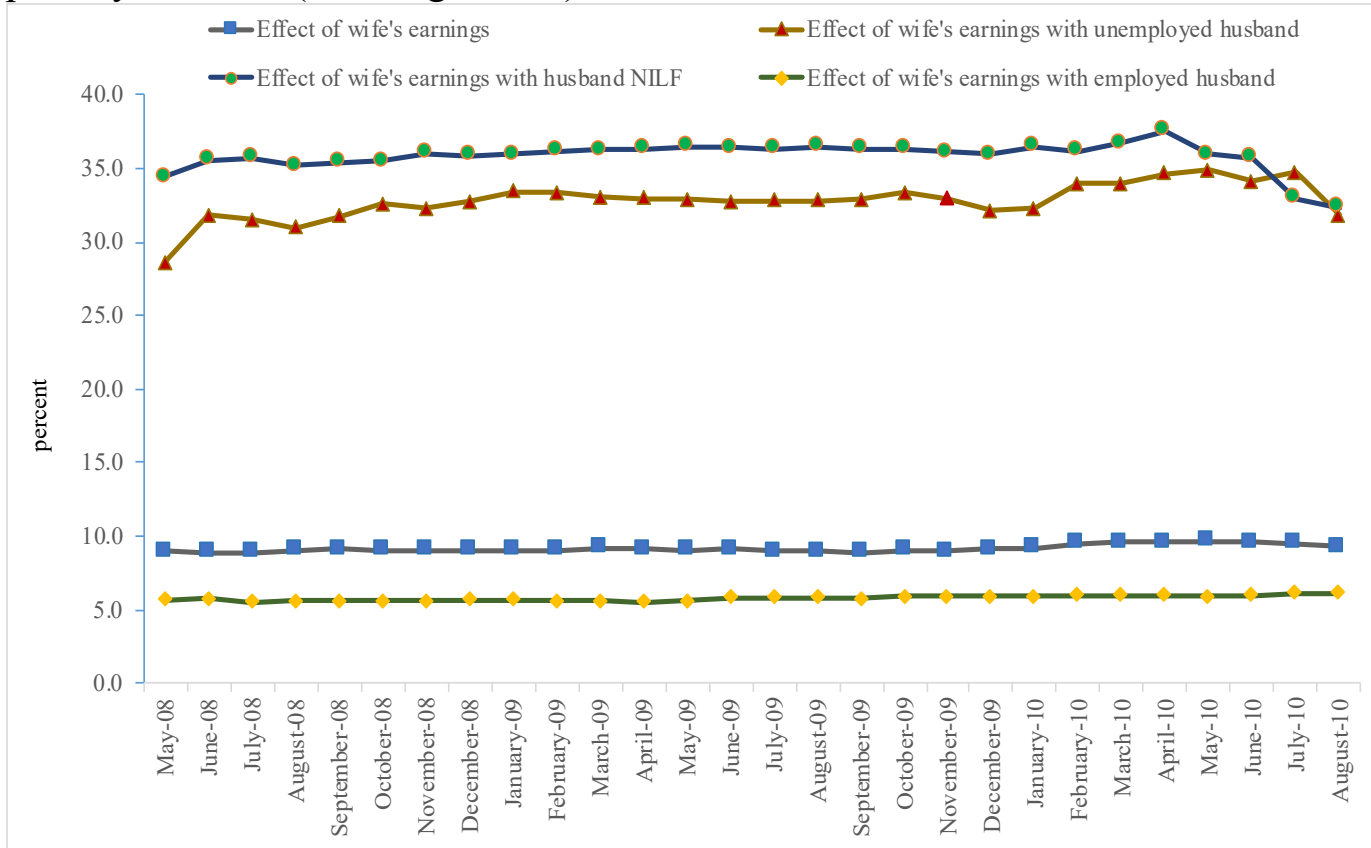

Source. Data analysis from wave 1 to wave 7 of SIPP 2008 and TAXSIM.

I consider our results' sensitivity to the inclusion of income or payroll taxes. In our sample, 92 percent of individual-months paid some form of tax on income. We observe how income tax affects the level of poverty, compared to the counterfactual of not paying any tax. The robustness check is applicable to the supplemental poverty measure, as it subtracts income tax from family income. In this analysis, we exclude the income tax liability information and look at the effect of the wife's working on family poverty based on the supplemental poverty measure. If the families are not paying income tax, they will have more disposable income for their consumption. Families' real income increases since they are not paying income tax and, hence, reduces poverty. 
Figure S8. Effect of wife's earnings on the level of poverty based on supplemental poverty measure (excluding income tax)

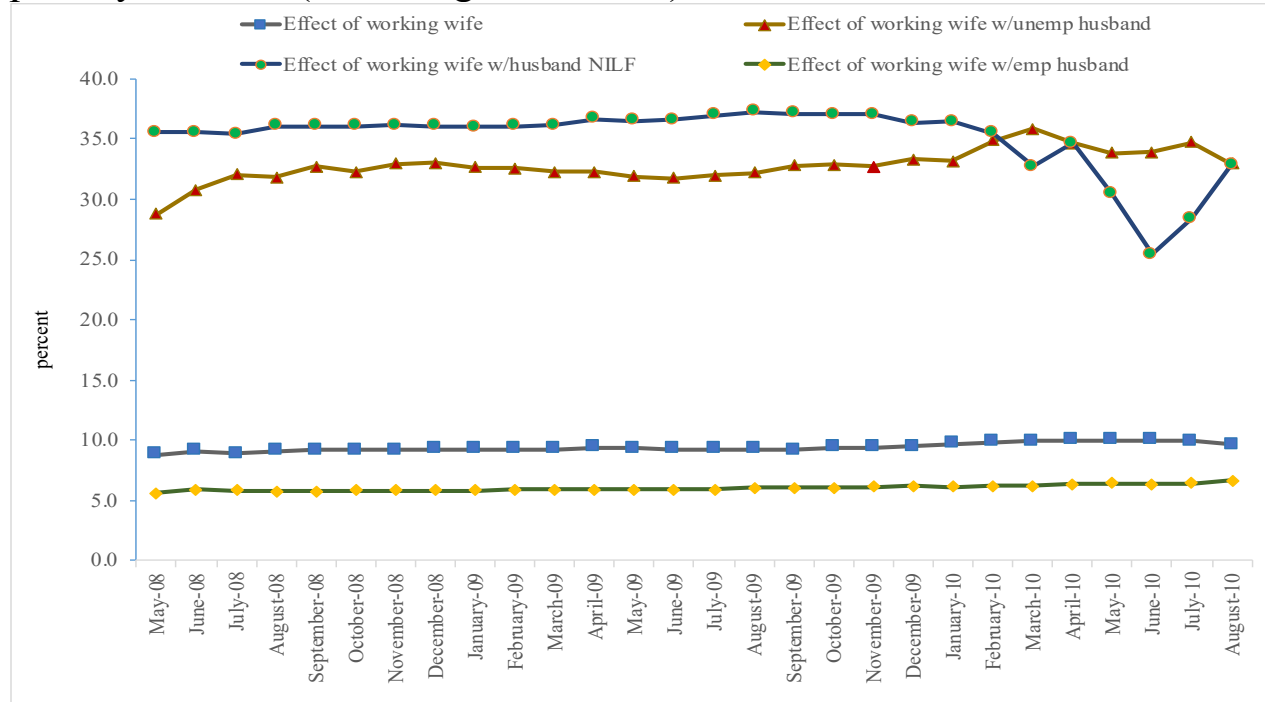

Source. Data analysis from wave 1 to wave 7 of SIPP 2008 and TAXSIM.

The wife's earnings effects remained whether or not we deduct income tax. In figure S8, we see that, on average, 9.4 percent of families escape poverty due to the wife's working. The average effects of wives working in households where the husband is unemployed, employed, and not in the labor force are 32.8 percent, 6.0 percent, and 35.1 percent, respectively. This finding leads us to conclude that income tax payment does not alter the effect of wife's working on family poverty. The decomposition analysis also displays little change in results when we do the analysis with and without adjusting for income tax liabilities (table S1 and S3). 


\section{CHAPTER 3: DID EXPANDING THE SUPPLY OF PUBLISHING OPPORTUNITIES INCREASE REPRESENTATION WITHIN THE ECONOMICS DISCIPLINE?}

\section{Introduction}

Publications are a lifesaver for people who are trying to establish their professional careers in academia. People start their tenure track faculty careers in academic positions and expect to move to a more settled tenured position. In this process, quality publication signals their future success in academia and thus are weighted more if they have required publications. However, publications from peer-reviewed journals are challenging and need a reasonable amount of time. Institutions determine a faculty member's fate; hence tenure based on publications in top-ranked journals are getting more demanding over time (Card \& DellaVigna, 2013; Heckman \& Moktan, 2020). In many academic institutions, authors are also rewarded financially for publishing in one of the top journals (Osterloh \& Frey, 2020).

The American Economic Journal (AEJ) series aimed to create new opportunities for publications for low-ranked institutions who can take the scope of publishing and can get success within the field in their publication capacity. Publication at the AEJs is prestigious since the four sub-journals gained high ranks in the field within a short period. Over the last 50 years, acceptance rates at top economics journals have fallen to $6 \%$ from $15 \%$. The time an economics paper typically spends at a given journal between submission and publication has more than doubled (Ellison, 2002; Galiani \& Gálvez, 2017). ${ }^{17}$ This paper aims to answer the causal question of whether publication opportunities create representation in economics.

\footnotetext{
${ }^{17}$ In this study, we use article and paper synonymously, which refers to the journal article.
} 
Success in academia relies on early success within a highly competitive space that might favor those with institutional resources or prestige. Given diminishing opportunities for publication in top-tier journals, the American Economic Association recognized a need for new journals where researchers and academics can publish their work. The American Economic Association began publishing four new journals in 2009, each using the title American Economic Journal, divided into sub-categories for different economics disciplines. ${ }^{18}$ The AEJ is a group of four reviewed academic journals with fields of Applied Economics, Economic Policy, Macroeconomics, and Microeconomics. Although this paper focuses on the economics discipline, the lessons are broadly applicable to other fields, where early success has been causally linked to long-term research productivity (Bol, de Vaan, \& van de Rijt, 2018).

This paper examines the impact of introducing the AEJs on academics' representation at lower-ranked institutions in academic economic publishing. As background, economics frequently refers to a set of "Top 5" journals representing the field's highest level. ${ }^{19}$ High-quality articles that are rejected from these journals are often published in the next "tier" of journals. The introduction of the AEJs was specifically designed to be "below" the "Top 5" but above these competitor journals..$^{20}$

There is growing literature that deals with the publication process and its consequence in the economics discipline (Akerlof, 2020; Anauati et al., 2020; Card \& DellaVigna, 2013; Ellison, 2002; Heckman \& Moktan, 2020). In these studies, scholars

\footnotetext{
${ }^{18}$ AEJ: Applied Economics, AEJ: Economic Policy, AEJ: Macroeconomics, and AEJ: Microeconomics. ${ }^{19}$ The "Top 5" include: American Economic Review, Econometrica, Journal of Political Economy, Quarterly Journal of Economics, and Review of Economic Studies.

${ }^{20}$ As a specific example, prior to 2009, an applied microeconomist might attempt to publish in a "Top 5" journal, and upon rejection, submit to the Journal of Human Resources (JHR). Now, the same author might use this rejection as an opportunity to publish in "American Economic Journal: Applied Economics", with JHR an ultimate venue only after this article was rejected from the AEJ.
} 
are investigating how the publication process was leading and reshaping the economics discipline. They are also giving their policy suggestions for the future. However, we did not find papers that looked at how introducing new journals influences the convoluted economics journal publication issues. We need to know how a brand new journal can solve some recurring problems. Does the brand new journal create some room for new scholars in the field?

The study examines whether the introduction of the AEJs has created new publication opportunities for scholars. To test this research question, we look if the journals ranked lower than the AEJs - which are still competitive and hard to get into become slightly less competitive. We would observe this if scholars from lower-ranked institutions are increasingly able to get their articles in these journals. We can observe this by the articles average institutional ranking of the authors that we can track over time. We test this empirically because it is not necessarily the case that these journals would get less competitive. To secure tenure positions, scholars need to publish an adequate number of high-quality articles. It could be the case that people who publish in AEJs would still publish papers in these other journals.

The results of this study provide some preliminary or weak evidence of causality. The analysis uses a difference-in-difference (DID) setting accompanied by the event study design. The study contributes to our understanding of new journals and how that can make a difference in economics's existing publication settings. Journal publication in economics is considered more difficult than most other disciplines, based on the lag between submission and publication and acceptance rate. 
The remainder of this paper proceeds as follows. Section two surveys existing literature on journal publication in economics. Section three deals with the background of the American Economic Journal series. We get an idea of the need for a new journal in economics in this section. Section four examines the data collection process. In this section, we discuss how we collect data and prepare our data for Stata. Section five discusses the methodology of the difference-in-difference and event study design. In section six, we describe the results of our analysis. We conclude with discussion and policy implications in the last section.

\section{Literature Review}

Economics is an unusual discipline among all the disciplines as it dedicates a set of a narrow list of top journals where scholars are competing to publish their research (Gibson et al., 2014). Economics emphasizes journal publication in a few high-ranked journals (Gibson, 2014; Heckman \& Moktan, 2020). This concentration of publications has intensified mostly throughout the last decade (Card \& DellaVigna, 2013). Publication in the top five economics journals works as a strong signal and influence for tenure decisions and transition rates to tenure (Heckman \& Moktan, 2020).

The scholarship of the economics's goal at its current state promotes careerism over creativity (Heckman \& Moktan, 2020). Scholars are emphasizing securing tenure positions, and they are putting less time into fundamental economic problems. Publication of economics journals has shifted to overly complicated or challenging papers, and journals are not interested in publishing soft papers. Akerlof (2020) describes this as the "sins of omission," which ignores the importance and valuable topics and 
problems that economics requires to address. Top economics departments consider tenure or promotion for those who were able to publish in the top five journals. However, it has been observed that a substantial number of influential papers are published in other journals, not in the top five (Heckman \& Moktan, 2020). In fact, even salaries are also tied with the journal productivity at the top five journals. Social ties between authors of journal papers and journal editors enhance the publications process where authors took advantage of the connection (Colussi, 2018; Goyal et al., 2006). In about $43 \%$ of published papers in the top four leading economics journals, authors of articles, and editors had some form of social connection during 2000-2006 (Colussi, 2018). ${ }^{21}$

The publication process of the top economics journal papers has been lengthened substantially over the last three decades (Ellison, 2002). Various factors contribute to this prolonged process. Papers are getting complicated, require extensive reviewing, and have established a democratic review process requiring more time for review. However, most of the slowdown remains unexplained. There are two types of delays in the publication process, including processing delays, which reflect time between submitting a paper and editors' decision after reviewing the paper, and publication delays between accepting a paper and its publication (Trivedi, 1993). Publication delays are prevalent in economics compared to science, technology, and medical (STM) fields (Björk \& Solomon, 2013). The average delays generally account for 18 months instead of 9 months average delay in chemistry in business or economics fields. From submission to acceptance, the review times in the top 25 economics journals require an average of 16.5 months (Ellison, 2002). In other statistics, the review time for nine economics journals has increased by $185 \%-$

${ }^{21}$ The top four journals consist of the American Economic Review (AER), Journal of Political Economy (JPE), Econometrica, and Quarterly Journal of Economics (QJE). 
from 6.1 months in 1970 to 17.3 months in 1999. In the 1960s, the author's requirement to "revise and resubmit" a paper was fairly rare, and rejection from a journal was uncommon (Akerlof, 2020; Ellison, 2002).

The number of submitted papers in the top five economics journals has substantially increased (Card \& DellaVigna, 2013), with high rejection rates and delays in the refereeing process (Ellison, 2002; Heckman \& Moktan, 2020). To ensure paper quality, editors are now using more referees than in the past (Heckman \& Moktan, 2020). The top five journals' acceptance rates have decreased from 15\% in 1980 to $6 \%$ in 2012 (Card \& DellaVigna, 2013). Publications in the top economics journals are increasingly demanding and require more time than in the past. As a result of this, high-profile senior authors with renowned institutional affiliations often publish their papers as working papers. They are less interested in publishing their papers in top-ranked economics journals (Ellison, 2011; Heckman \& Moktan, 2020). Due to the high growth of internet connectivity, high-profile authors disseminate their papers directly to readers. This auxiliary publication process dampens the market signal of the top five. These papers are highly cited but not published in the top five journals (Heckman \& Moktan, 2020). Despite high citation counts by papers published in the top five journals and longer life cycles, their influence is overestimated (Anauati et al., 2020).

Card and DellaVigna (2013) have identified nine essential facts for the top five journals. First, the annual submissions to these top five journals were double in 2012 compared to 1990 (Anauati et al., 2020). Second, the total number of articles published annually has declined from 400 in the 1970 s to 300 in 2012. Consequently, the journal acceptance rate has declined from $15 \%$ to $6 \%$, which puts pressure on young 
professionals. Third, the American Economic Review accounts for $40 \%$ of all published articles in the top five journals. This share has increased from $25 \%$ in the 1970 s. Fourth, currently published journals have an increased length of three times higher than the 1970s, which implies greater space limitations for articles. Fifth, the average number of authors has increased to 2.3 per article from 1.3 in 1970. Sixth, articles published in the top five journals have a high citation record (Anauati et al., 2020). Seventh, the rankings of journals remain about the same within the top five. The only exception is the Quarterly Journal of Economics, which peaked from the fourth position during the three decades. Eighth, lengthy papers have many more citations and coauthors. Ninth, papers related to development and international economics are getting more citations, and econometrics and theoretical papers are getting fewer citations.

Even in the top economics departments, some females require more top-five publications than their male colleagues. Despite the small sample size, male faculties require two top 5 publications, and female faculties require three top-five publications (Heckman \& Moktan, 2020). We usually see discrimination based on external characteristics like race, gender, etc. There is "alphabetical discrimination" in academic success in economics (Einav \& Yariv, 2006). In a collaborative paper in economics, authors' names are in alphabetical order and get some privilege in the first place. When people cite papers, they use the first author's last name with "et al." if the paper has more than two authors. The alphabetical discrimination also favors economists likely to receive the Clark Medal and the Nobel Prize.

The fate of an article sometimes depends on when the paper was published. A "citation trap" exists in academic paper publications depending on when (month) the 
paper was published (Ma et al., 2019). If a paper is published between October to December, the paper is $18.5 \%$ less likely to be cited relative to a paper published in other months of the year.

Publications are the central blood circulation system in academia. However, sometimes authors are not treated well enough for their scholarly contribution. The author of a paper can publish a paper if she shows intellectual prostitution to the journal editor (Frey, 2003). People should treat economists as artists and journals who need to understand creativity's value as an intellectual field. Mutual respect may encourage everyone to work what the field deserves.

\section{Background on the American Economic Journal}

The American Economic Association (AEA) started to publish the American Economic Journal series in 2009. AEJ is a group of four reviewed academic journals with fields of applied economics, economic policy, macroeconomics, and microeconomics. Each of the journals starts with the prefix American Economic Journal with a field name attached.

There was a rejuvenated need for new journals in economics where researchers and academicians could publish their research output. Among many reasons for the new publication avenues in economics journals, these two are prominent for the history of AEJ. Most other academic societies publish more than three journals, and adding more journals can ensure the diversity of papers and editors. The American Economic Review 
was too populated as it was a sole publication from the American Economic Association. $^{22}$

The Ad Hoc Committee on new Journals chaired by Robert Hall recommended to the Executive Committee in April 2006 that the association start four aggregated field journals. This recommendation came following a three-year study and discussion of the subject. The publication start date was set at 2009, and each published in four issues per year. The four new field journals of AEJ are not subsidiary journals to the AER. The AEJ journals are lower ranked than the AER since the beginning of AEJ. At that time, the AEA president, George Akerlof, appointed a search committee for the new journals' editors. The Executive Committee of the AEA voted to initiate the new field journals and name them: (i) American Economic Journal: Macroeconomics, (ii) American Economic Journal: Microeconomics, (iii) American Economic Journal: Economic Policy, (iv) American Economic Journal: Applied Economics.

AEJ: Macroeconomics primarily would include macroeconomics, monetary economics, international finance, aggregate aspects of development, economic growth, finance, and comparative economic systems. The primary focus of AEJ: Microeconomics includes microeconomic theory, corporate finance, industrial organization, micro theory aspects of economic development, and micro aspects of international economics. AEJ: Economic Policy has a publication focus on public economics, urban and regional economics, public policy aspects of health, education, welfare, law and economics, economic regulation, and environmental and natural resource economics. AEJ: Applied

\footnotetext{
${ }^{22}$ The American Economic Association has published the Journal of Economic Literature and the Journal of Economic Perspectives. However, in general, these journals commission articles and are not open to submissions.
} 
Economics concentrates papers on labor, demography, empirical micro developments, health, education, and welfare economics.

\section{Data}

Data for this study come from Scopus, which manages the abstract and citation database of peer-reviewed literature and is hosted by Elsevier. ${ }^{23}$ This gives us the publication detail of an article, including the name of the article, author(s) name, number of citations. The rank for economics journal is published by IDEAS/RePEc (IDEAS/RePEc, n.d.) and updated periodically. In our database, we link the economics journal ranking with the journals at Scopus. For this analysis, we consider the top 120 economics journals, journal rank rated from 1, highest, to 120 lowest. This research focuses on the top 120 journals for two reasons. First, we are interested in seeing the impact of the American Economic Journal on other competing journals, and the ranking of AEJ varies widely. The four AEJs ranks are macroeconomics (18), applied economics (25), economic policy (52), and microeconomics (116). This study needs to include all the journals that are within the range of 116 at the least. To manage the rank of microeconomics, we consider journals that rank between 1 to 120 . Second, there is an implicit rule of thumb in many economics departments to publish in the top 100 journals for tenure track positions. Publication in the top 100 journals is considered their signal for future productivity and scholarly contribution. The institutions try to predict the future success of a faculty by their publication capacity. Despite economics department policy

\footnotetext{
${ }^{23}$ This research has started with a broader context of journals by considering 4,000 journals in the economics discipline. Some of the publications are dated back to the 19th century. To address the research question, we worked with the top 120 journals and the papers published after 1990.
} 
statements to the contrary, they prefer to see the papers published in the top rankings journals (Heckman \& Moktan, 2020).

In this study, the unit of analysis is an article, and the article is assigned with the average institutional adjusted ranking of the author that we track over time. We collected data based on parameters of the journal-title, year, volume, issue, article number, start and end page, page count, cited by, authors name, authors id, affiliation, funding, publisher, and document type. The current analysis is limited to publications from 1991 to 2019. We have excluded earlier publications prior to 1991 as they are not relevant to the current analysis. In the analysis part, we check the main estimates' robustness by limiting the data from 2000 to 2019 (results are not presented in this paper). There is a change in policy in 2009 when the American Economic Journal series started publishing. The year 2009 is important due to expected changes since the start of the AEJ series publication. This works like a change in policy in 2009 for the difference-in-difference model.

The following steps elaborate on the procedure to get the data for difference-indifference analysis. In step 1, we collect the top 120 economic journal article records from Scopus. We downloaded Scopus data on May 14, 2019. We import files downloaded from Scopus for individual economics journals. We arranged publication history by different journals and authors along with other information. Afterward, we kept only those articles that are published from 1991 onward. The year 1991 will provide a good reference year to start. Authors who have published papers from 1991 to 2008 will provide us the control group information in this research. Seven journals are not imported (rankings of 22, 29, 43, 65, 70, 74, 76) as these are proceedings of various U.S. federal 
reserve banks (6) or working papers (1). Proceedings, Federal Reserve Bank of San Francisco (22), Proceedings, Federal Reserve Bank of Cleveland (29), Quarterly Review, Federal Reserve Bank of Minneapolis (43), Economic Policy Review, Federal Reserve Bank of New York (65), Journal of Global Economic Analysis, Center for Global Trade Analysis (70), BIS Quarterly Review, Bank for International Settlements (74), Proceedings, Board of Governors of the Federal Reserve System (U.S.) (76). There were 142,301 papers published in 120 journals from 1991 to 2019 . The data are arranged in one row per article. A single paper or article may have multiple authors in this data. In table 1, we see the percent of articles published in different years. The year 2019 shows a sharp fall since we collected data for the first five months. In most other years, we see an increasing trend of article publication. There is an increase in the supply of papers from 1996, and we see until 1995, there was less than $2 \%$ of papers published annually.

Table 1 provides some descriptive statistics for the data. On average, over the full sample period, 4,932 articles were published annually from 1991 to 2018 in the 120 selected journals. We also see an increasing trend of publication for articles. Some journals have increased their frequency of publication and started accommodating more articles in a publication. In fact, it is the case that we see a few new journals in these years for economics.

Table 1. Descriptive Statistics

\begin{tabular}{|c|r|r|r|r|r|c|}
\hline \multirow{2}{*}{ Year } & \multicolumn{3}{|c|}{ Articles } & \multicolumn{3}{c|}{ Citations } \\
\cline { 2 - 8 } & Numbers & $\begin{array}{c}\text { Percent } \\
(\%)\end{array}$ & $\begin{array}{c}\text { Percent } \\
\text { Change } \\
(\%)\end{array}$ & Number & $\begin{array}{c}\text { Percent } \\
(\%)\end{array}$ & $\begin{array}{c}\text { Percent } \\
\text { Change } \\
(\%)\end{array}$ \\
\hline 1991 & 2119 & 1.49 & NA & 1485 & 0.02 & NA \\
\hline 1992 & 2239 & 1.57 & 5.66 & 13430 & 0.22 & 804.38 \\
\hline
\end{tabular}




\begin{tabular}{|r|r|r|r|r|r|r|}
\hline 1993 & 2299 & 1.62 & 2.68 & 33185 & 0.54 & 147.10 \\
\hline 1994 & 2555 & 1.80 & 11.14 & 54351 & 0.88 & 63.78 \\
\hline 1995 & 2613 & 1.84 & 2.27 & 82551 & 1.34 & 51.88 \\
\hline 1996 & 3834 & 2.69 & 46.73 & 110300 & 1.79 & 33.61 \\
\hline 1997 & 3773 & 2.65 & -1.59 & 116644 & 1.89 & 5.75 \\
\hline 1998 & 3855 & 2.71 & 2.17 & 150639 & 2.44 & 29.14 \\
\hline 1999 & 3723 & 2.62 & -3.42 & 154810 & 2.51 & 2.77 \\
\hline 2000 & 3778 & 2.65 & 1.48 & 158342 & 2.57 & 2.28 \\
\hline 2001 & 3973 & 2.79 & 5.16 & 159969 & 2.59 & 1.03 \\
\hline 2002 & 4261 & 2.99 & 7.25 & 175574 & 2.84 & 9.76 \\
\hline 2003 & 4611 & 3.24 & 8.21 & 186300 & 3.02 & 6.11 \\
\hline 2004 & 4779 & 3.36 & 3.64 & 205294 & 3.33 & 10.20 \\
\hline 2005 & 4862 & 3.42 & 1.74 & 229874 & 3.72 & 11.97 \\
\hline 2006 & 5149 & 3.62 & 5.90 & 264640 & 4.29 & 15.12 \\
\hline 2007 & 5479 & 3.85 & 6.41 & 284719 & 4.61 & 7.59 \\
\hline 2008 & 6049 & 4.25 & 10.40 & 284739 & 4.61 & 0.01 \\
\hline 2009 & 6162 & 4.33 & 1.87 & 291003 & 4.71 & 2.20 \\
\hline 2010 & 6192 & 4.35 & 0.49 & 294313 & 4.77 & 1.14 \\
\hline 2011 & 6340 & 4.46 & 2.39 & 302081 & 4.89 & 2.64 \\
\hline 2012 & 6580 & 4.62 & 3.79 & 305662 & 4.95 & 1.19 \\
\hline 2013 & 7106 & 4.99 & 7.99 & 315770 & 5.12 & 3.31 \\
\hline 2014 & 7196 & 5.06 & 1.27 & 321068 & 5.20 & 1.68 \\
\hline 2015 & 6738 & 4.74 & -6.36 & 321633 & 5.21 & 0.18 \\
\hline 2016 & 6719 & 4.72 & -0.28 & 324102 & 5.25 & 0.77 \\
\hline 2017 & 7194 & 5.06 & 7.07 & 334021 & 5.41 & 3.06 \\
\hline 2018 & 7900 & 5.55 & 9.81 & 346709 & 5.62 & 3.80 \\
\hline 2019 & 4223 & 2.97 & -46.54 & 349745 & 5.67 & 0.88 \\
\hline
\end{tabular}

Notes: Second column refers to the number of journal articles published in 120 selected journals in respective years. Column 3 identifies the percent of articles been published in a year. The fourth column represents the percent change in articles relative to the previous year. A single journal article may have multiple authors, if applicable. The fifth column refers to the number of citations that these journal articles get in their respective years. In the sixth column, the percent of citations in a year refers to percent relative to total citations from 1991 to 2019. The last column refers to the percent change in citations relative to the previous year. *Data for 2019 contains the first five months of the year.

In table 1 , in the last column, the percent of citations in a year refers to percent relative to total citations from 1991 to 2019. From 1991 to 2018, we see an average citation of 42.2 per article. There is a maximum citation of 9,365 for a single article, and 
some articles do not get any citation after publication. Overall, we also see an increasing trend of citations measured by the percent of citations in respective years. The articles are more impactful in recent years than in the past. Due to the increasing accessibility of technologies in recent years, researchers can search a paper and cite a paper that they need.

In step 2, we reshaped journal articles into one row per author-article from one row per article. The goal here is to identify each author with their publication. Also, our interest is to append the institutional ranking of the author's affiliated institution. This gives us an idea about the authors' departmental ranking with the journal article. ${ }^{24}$ As a result of reshaping, we see a two-fold increase in total observations in our data window from 1991 to 2019. Percent of journal papers is in appendix figure A1, where we see after reshaping into one row per author-article. The percent is defined by the percent of observations for respective years relative to the total number of observations from 1991 to 2019 and represented by the line with a circle marker. This follows an increasing trend over the years. The second line (square marker) represents the percent change of articles relative to the previous year. In most of the years, we see an increasing number relative to previous years. In a few years, we see there is a sharp increase. Two factors can

\footnotetext{
${ }^{24}$ To make it one row per author-article, we have a unique id with paper's unique identifier. Scopus assigns this id in its database to identify the unique academic work, and they call this electronic identification (EID). Scopus seems to work with its own digital identifier, similar to the digital object identifier (DOI) used in many journals. We consider authors id, file, volume, issue, and page start for unique id. After reshaping the one row per author article, we have 293,461 observations where each observation shows one row per author-article. In this data, a single article appears multiple times, depending on the number of authors. However, if one article has a sole author, it appears once in the dataset. In other words, this has a single observation for the article. If 5 authors write an article, the journal article will appear five times in our dataset after reshaping. For that particular journal article, we see an increase in observation from one to five.
} 
contribute to this sharp increase: an increase in the number of articles and an increase in co-authored articles.

In step 3, we matched the reshaped data, which contains one row per authorarticle, to the institutional ranking by authors' affiliations. The matched dataset will have institutional ranking in addition to other information that we get in step 2 . In the reshaped dataset, we have journal rankings from 1 to 120 , and we also have authors' institutional affiliation information. The institutional ranks are directly from the economics department rankings in various institutions and mostly from the universities. The institutional rank contains 287 different institutions, mostly with economics departments where 1 is the highest and 287 is the lowest. In the dataset, we have 120 journals with their rank. Departments whose rankings are 120 and above are set to 120 . To avoid outliers and missingness, authors whose institutional affiliation was greater than 120 or who had no institutional affiliation (i.e., lower-ranked universities; unaffiliated jobs such as government or research organizations) were assigned a value of 120 . In other words, the value 120 for institutional affiliation represents the maximum ranking possible for our dataset, which states the person or author is from a low-ranked institution. In the dataset, we create a unique affiliation id by using the author's id. In this process, we remove some extraneous strings that do not match our observations.

To identify the departmental rankings between the journal articles and IDEAS/RePEc, we used Stata's matchit program. These were contained in string variables that may include different spellings and abbreviations or other differences that make matching imprecise. This program matches two datasets based on similar text patterns and uses string variables, with the closeness of matches specified by the 
similarity scores, known as similscore. The scores vary between 0 to 1 , where 1 means a perfect match in the matching results.

In step 4, we examine the accuracy of matching that we performed in the 'matchit' procedure. Now, we keep only the highest similscore for each journal affiliation. Data derive from a match of institutional rank taken from IDEAS/RePEc linked to Scopus data using Stata's 'matchit' procedure. Appendix figure A2 presents a histogram that shows the highest similarity score per institution, conditional on a similarity score higher than 0.5 . After producing these results, we selected a random sample of 25 matches for each bin of 0.05 similscore points (i.e., $0.50-0.549 ; 0.55-0.599$ ) and used human judgment to verify the accuracy. Appendix figure A3 shows the ability of Stata's 'matchit' procedure to link the author's stated affiliation to institutional rank. We observe a generally linear relationship between similscore and match accuracy, with similscore of 0 being associated with almost completely incorrect matches and a similscore of 0.9 being judged close to completely accurate. We see over $60 \%$ of observations are correct match with an estimated matching probability over 0.65 .

In step 5, we work with the matched data from the third step of this section, where we match the reshaped data to institutional ranking. This study analyzes the impact of introducing the AEJs on academics' representation at lower-ranked institutions in academic economic publishing. As background, economics frequently refers to a set of "Top 5" journals representing the field's highest level. High-quality articles that are rejected from these journals are often published in the next "tier" of journals. The introduction of the AEJs was specifically designed to be "below" the "Top 5" but above these competitor journals. Appendix figure A4 shows the authors' average institutional 
rank per journal publication considering institutional ranking from 1 to 287 . We see that the AEJs were indeed an attractive venue as scholars of more robust institutional rank chose to publish there; the average rank was slightly lower than in the "Top 5" journals but above the remaining journals considered part of the "Top 120" in economics.

In figure 1, we have the average institutional adjusted rank of authors per journal publication, considering the institutional ranking from 1 to 120 . Calculations used in this study are the average institutional rank per published journal article. In figure 1, the solid line represents "Top 5" economics journal. The deep dotted line represents AEJ, and the light dotted line represents the rest of the economics where we have 111 other economics journals. The American Economic Journal series includes all four journals in the top 120 journals: applied economics, economic policy, macroeconomics, and microeconomics. We also see that the AEJs were indeed an attractive avenue to publish papers as scholars of more robust institutional rank chose to publish there. The average adjusted rank was slightly lower than the "Top 5" journals but above the remaining journals of "Top 120" in economics. 
Figure 1. The average institutional adjusted rank of authors per journal article

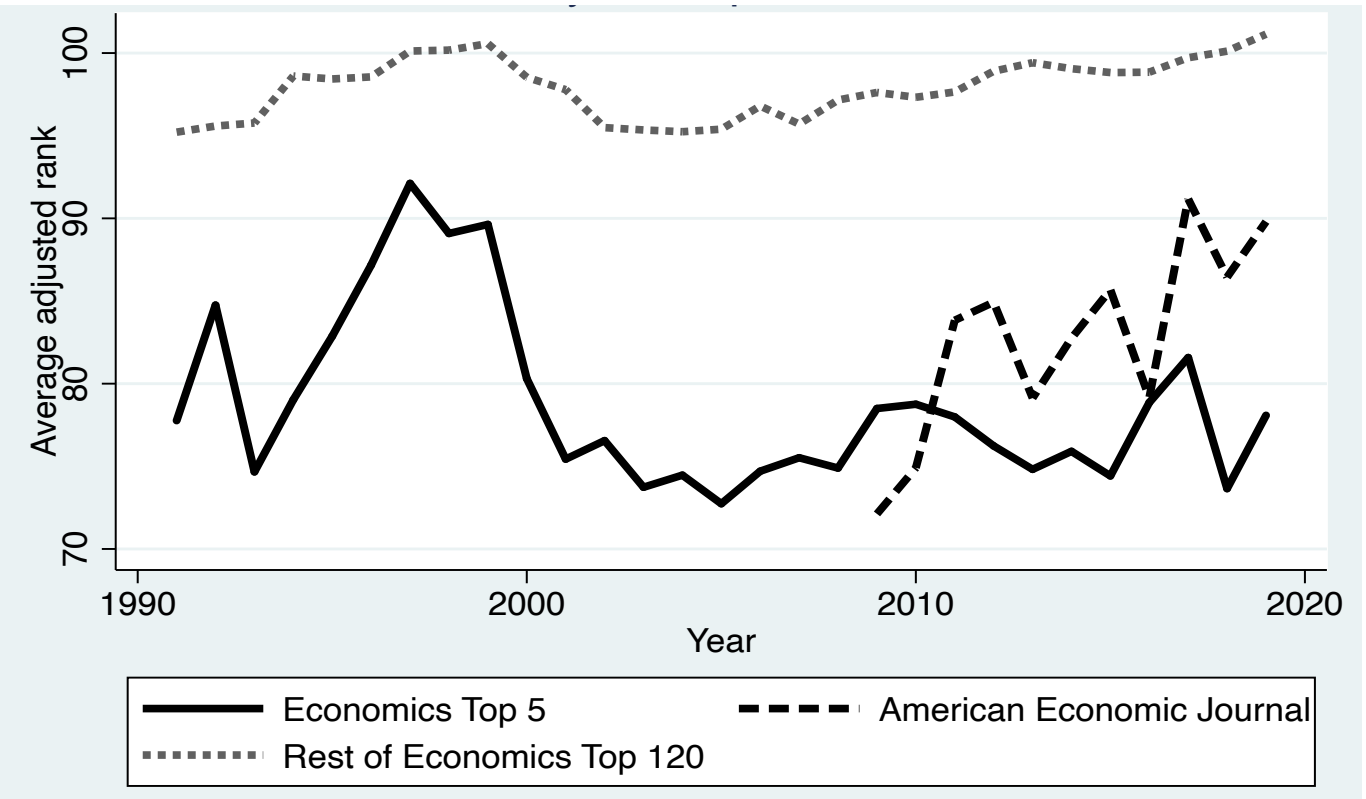

Notes: Data include all publications from the top 120 Economics journals, as measured by IDEAS/RePEc data and downloaded from Scopus in June 2019. Institutional rank was taken from IDEAS/RePEc and linked to Scopus data using Stata's 'matchit' procedure. Calculations are the average institutional rank per published journal article; to avoid outliers and missingness, authors whose institutional affiliation was greater than 120 or who had no institutional affiliation (i.e., lower-ranked universities; unaffiliated jobs such as government or research organizations) were assigned a value of 120. "Top 5" refers to American Economic Review, Quarterly Journal of Economics, Econometrica, Review of Economic Studies, and the Journal of Political Economy. The American Economic Journal category includes all four journals in the top 120 journals: Applied Economics, Economic Policy, Macroeconomics, and Microeconomics.

Figure 1 is comparable to appendix figure A4 in this study, with the only difference is the way we define the average institutional rank of authors. In figure 1, we defined the average institutional adjusted rank of authors by truncating the institutional rank from 287 to 120 . Since we have 120 journals rank in our dataset, we also consider 120 institutional ranks. However, in the appendix figure A4, we see the average institutional rank of authors per journal publication, and we did not truncate the institutional ranks. Two different average institutional rank measures per journal article (average institutional rank and average adjusted institutional rank) give us the same 
conclusion. We prefer to use the adjusted rank, which will be a better measure than the other one. The adjusted measure is precise and has less variability.

The citation of an article is important since we need to know how impactful the research was. Figure 2 shows the average citations of these early AEJ papers are comparable to those published in the "Top 5" economics journal. As expected, the average citations per journal publication declined for the three groups of journal articles as we moved in recent years. Old papers have more citations than new papers. The early papers from the AEJ series were able to get a good number of citations. The average number of citations for the AEJ series papers was always higher than the rest of economics' top 120 journal papers.

Figure 2. Average citations per journal publication

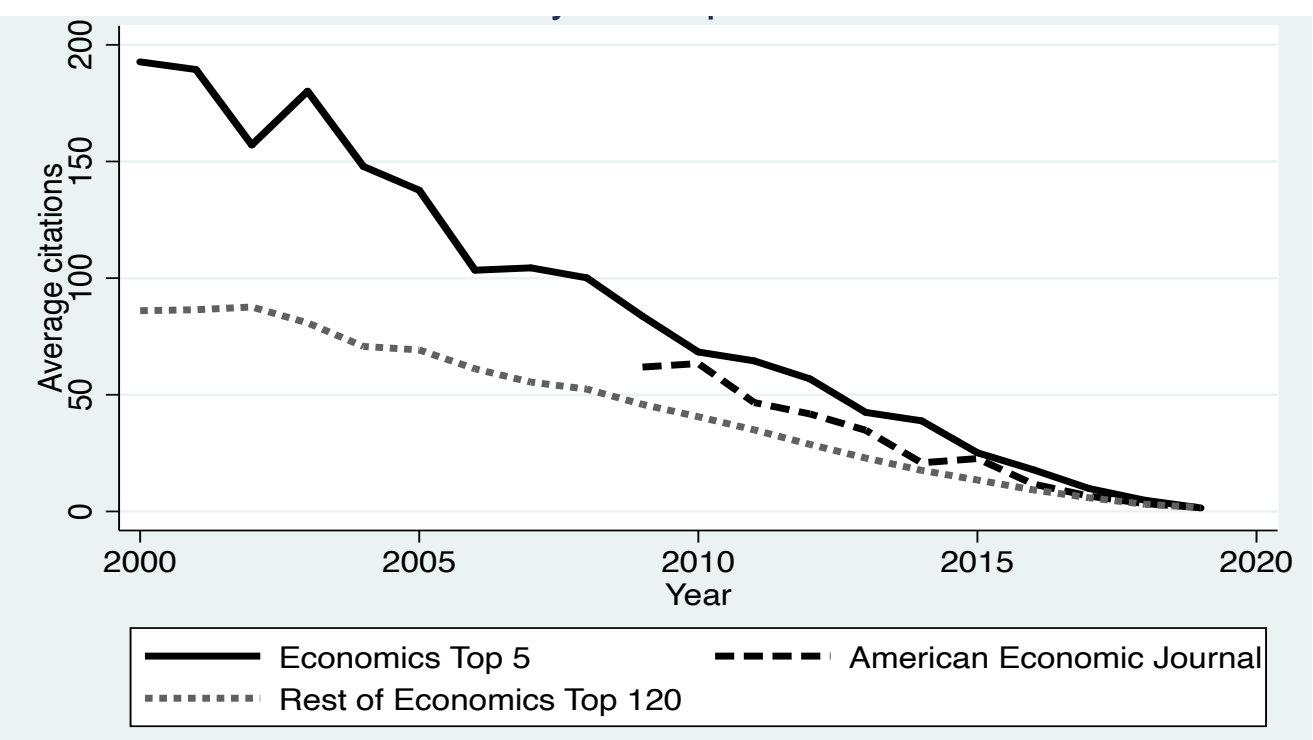

Notes: Data include all publications from the top 120 Economics journals, as measured by IDEAS/RePEc data and downloaded from Scopus in June 2019. Institutional rank was taken from IDEAS/RePEc and linked to Scopus data using Stata's 'matchit' procedure. Calculations are the average institutional rank per published journal article; to avoid outliers and missingness, authors whose institutional affiliation was greater than 120 or who had no institutional affiliation (i.e., lower-ranked universities; unaffiliated jobs such as government or research organizations) were assigned a value of 120. "Top 5" refers to American Economic Review, Quarterly Journal of Economics, Econometrica, Review of Economic Studies, and the Journal of Political Economy. The American Economic Journal category includes all four journals in the top 120 journals: Applied Economics, Economic Policy, Macroeconomics, and Microeconomics. 
In step 6, now we proceed to prepare our dataset to apply difference-in-difference in this study. We need observations for the treatment group and control group. We define three different tiers: tier 1, tier 2, and tier 3 based on journal groups. Tier 1 consists of journals from "top 5." Tier 2 represents the AEJ series journals. We call authors who have published papers in "tier 3" journals are the treatment group. The process follows the following steps. At the outset, we identify all the authors who have published in the "top 5" journals before the AEJ series of 2009. From these observations of authors, we also identify those authors who have published at least two papers in the "top 5." We argue here that "at least two papers" is a standard criterion to infer that the authors have a strong track record of publication in the "top 5" journals. To get the authors of the treatment group for tier 3, we identify authors, prior to the AEJ's what other journals they were publishing more frequently who also have at least 2 papers in "top 5." We get our treatment group observations which consist of authors of one row per author-article.

We want to limit our observations by the number of citations where we have already identified authors. As we mention in step 5, we to consider articles that are impactful research findings and measured by citations. In our dataset, the average citation of a paper is 42.2 . We set two thresholds for the number of citations, and the aim is to be conservative while setting the number of citations. Now, we identify articles with at least 200 citations. This process tells us that there are 28 journals where these authors have published their papers more frequently apart from "top 5." We call this more frequent publication since we have identified only those authors who have published their research at least 2 papers in the "top 5." These are our treatment group journals. These are the journals where selected authors published their papers more frequently. In this study, the 
unit of analysis is the authors' rank, and we get this by applying several conditions. We also consider a second selection process for tier 3 journals which is less conservative than the first. Here, we track those articles that have at least 100 citations. There are 48 journals where these authors are published more frequently, and we consider these journals as new tier 3 . The four AEJ series are our tier 2 journals. This is our change in program in this research. The introduction of AEJs is considered the study's interventions, and the interventions are as good as random. Appendix table A1 presents a list of journals used in this study with their groups as described.

\section{Methodology}

The difference-in-difference method is ideal where outcomes are observed for two groups for two different periods. Ashenfelter and Card (1985) popularized this method as a quasi-experimental research method. The difference-in-difference looks at the effect of a policy change in a period that separates two different groups. One group is exposed to the policy change and is known as the treatment group. On the other hand, the other group was not exposed to the policy change and is known as control. This counterfactual is important to make a causal inference.

This study uses a difference-in-difference framework, which empirically tests whether the American economic journals' introduction increased representation by institutional quality among elite but secondary journals that historically fell below the "Top 5." 25 In other words, this study analyzes the impact of the introduction of the AEJs in 2009 on academics' representation at lower-ranked institutions in academic economic

${ }^{25}$ Difference-in-difference is one of the popular and oldest quasi-experimental research designs dated back to Snow's (1855) study on a cholera outbreak in London (Goodman-Bacon, 2018). 
publishing. In essence, we believe that representation in Economics elite "Top 5" journals will be unaffected by introducing the AEJs and serving as a control group to measure overall changes in who publishes in elite economic journals over this period. In the difference-in-difference model, the control group works as a counterfactual for the model, and we need to ensure that we have a good control group for the analysis. The AEJ then serves as the "treatment" condition, and we will test whether this new AEJ tier created space for those in lower-ranking institutions to publish in high-quality but slightly lower-ranked journals. The results from figure 1 offer some speculative evidence that this is indeed the case, with average ranking in the "Top 5" unchanged after 2009. However, institutional ranking in other top 120 journals was increasing during this time.

Next, we proceed to our main model. We first examine a linear difference-indifference model. In this study, a difference-in-difference analysis empirically tests whether the introduction of the AEJ journals increased representation by institutions of lower quality among elite but secondary journals that historically fell below the "Top 5." The identifying assumption for the DID method is whatever happened to the "Tier 1" journals (control group) over time is what would have happened to the "Tier 3" journals (treatment group) in the absence of the AEJs (program).

The most parsimonious version of this linear difference-in-difference framework can be represented as the following equation:

$$
\begin{aligned}
\left.y_{i t}=\alpha+\beta_{1} * \text { Treatment }_{i t}+\beta_{2} \text { post }_{i t}+\beta_{3} \text { Treatment }_{i t} * \text { post }_{i t}\right) \\
+\varepsilon_{i t} \ldots \ldots \ldots(1)
\end{aligned}
$$

where $y_{i t}$ is the outcome of interest, and in this study, it is measured by the articles with the average institutional adjusted ranking of the authors. The articles adjusted 
institutional rank of authors are defined by the rank of authors institutional rank with the journal rank. In our dataset, the observations are per author-article where we may get an article authored by multiple authors. The dummy variable Treatment $_{i t}$ is the tier 3 journals capture possible differences between the treatment and control groups prior to the publication of AEJs. In this study, there are 2 different treatment groups based on the number of citations that we set. The time dummy post ${ }_{i t}$ captures the possible change in the outcome variable in the absence of a policy change. The coefficient of interest is the estimate of $\beta_{3}$, an interaction between Treatment $_{i t}$ and post ${ }_{i t}$, and known as a difference-in-difference estimate.

In our next step, we include fixed effects in our model, and in this case, we use the journal fixed effect. The fixed effect version of this linear difference-in-difference framework can be represented as the following equation:

$$
\begin{aligned}
y_{i t}=\alpha+\beta_{1} & \text { Treatment } \left._{i t}+\beta_{2} \text { post }_{i t}+\beta_{3} \text { Treatment }_{i t} * \text { post }_{i t}\right)+\theta_{j} \\
& +\varepsilon_{i t} \ldots \ldots \ldots(2)
\end{aligned}
$$

In equation 2 , we have a similar description as in equation 1 above. $\theta_{j}$ identifies the journal fixed effect. In other words, the variable $\theta_{j}$ captures all unobserved, timeconstant factors that affect the authors' rank of $y_{i t}$. The journal fixed effect does not change over time and $\theta_{j}$ is fixed over time. The journal fixed effect represents all factors affecting the articles average adjusted institutional rank of authors that do not change over time.

The standard way to evaluate interventions such as the causal effect of publication opportunities to a new journal can be evaluated by the difference-in-difference method. DID method follows a version of panel fixed effects and can also be used with repeated 
cross-sections (Cunningham, 2018). The DID eliminates both the selection bias and the effect of time. The first difference eliminated the unit-specific fixed effects, and after getting the difference-in-difference, it gives unbiased estimates. There are two key assumptions to apply a standard difference-in-difference model. First, there are no timevariant unobservables. Second, the omitted variable bias is the same for all units. The second assumption is known as the parallel trends assumption in the DID model. The simple differencing approach in the DID model has many advantages. DID can control for other variables, and this may reduce the standard errors.

The difference-in-difference analysis relies on data from all economics journals ranked up to 120 and linked to the economics departments ranking. We define authors rank who publishes at the tier 1 journals as the control group for the difference-indifference model. Tier 1 includes the top 5 economics journals. Tier 3 journals is considered as the treatment group. In one measure, the process gives 28 journals that constitute tier 3 . The second measure gives 48 journals that are in tier 3 . The procedure of getting these treatment journals is explained in the data section. The identifying strategy requires a program effect, and in this study, the introduction of AEJ publication is the program that has some potential implication to the economics discipline, by and large.

The difference-in-difference study design has two different statistical concerns (Gurantz, 2020). The first challenge is whether the control group of tier 1 journals works as a proper counterfactual for tier 3 of treatment journals in the absence of AEJs. We need to check whether tier 1 and tier 3 have similar pre-treatment trends. The event study design helps to analyze the trends by interacting the treatment (tier 3) with each year 
dummy in the pre-treatment period where we exclude the year 2008 as this is our baseline year. This follows the following equation:

$$
y_{i t}=\alpha+\sum_{t=1991}^{2019}\left(\beta_{1 t} * \text { Treatment }_{i t} * \delta_{t}\right)+\theta_{j}+\delta_{t}+\varepsilon_{i t} \ldots \ldots \ldots
$$

I include both journal $\left(\theta_{j}\right)$ and year $\left(\delta_{t}\right)$ fixed effects. The equation tests for the treatment effect in each year. The difference-in-difference coefficients should be close to zero to support that there was a pre-treatment trend. To check the difference-in-difference model's common trend assumption, we perform an event study design with two different time periods of 1991 to 2007 and 2009 to 2019 . We have omitted the year 2008 since the policy change took place in 2009. An event study design is accompanied by the difference-in-difference method for program evaluation in microeconomics. The event study design is a powerful tool for analyzing policy change (Sandler \& Sandler, 2014).

Statistical inference for the estimates is our second statistical concern. A potential threat is the outcomes are often serially correlated due to many years of data, and the standard errors may be inconsistent (Bertrand et al., 2004). The conventional differencein-difference standard errors are underestimated and give us misleading inferences on rejecting the null hypothesis. In this study, the estimation process takes into account cluster standard errors at the journal level. As an alternative, we use Stata's default bootstrapping method for standard errors without regard to clustering. The bootstrapping method of estimating standard error attempts to account for the autocorrelation of data. The number of journals in this study is large enough to address the data's autocorrelation, and bootstrap will work well (Bertrand et al., 2004). 


\section{Results}

\section{Main Treatment Effects}

The AEJs publication increases the gap in institutional ranking between authors in the "Top 5" journals and authors in high-quality but slightly less competitive journals. The policy leads high-quality journal articles to have authors from lower-ranked institutions compared to "Top 5." The increment is by 2.9 points with the control of 28 journals, in table 2 . This is significant at $p<0.01$. A new journal works as an opportunity of publishing articles for authors from low-rank institutions in economics. When we consider a control group of 48 journals, the AEJs publication helps to increase the articles average institutional adjusted rank by 2.7 points. This is also significant at $p<0.01$. In table 2 , we have the event study design using equation 3 accompanied by the differencein-difference with different years. The year 2008 has been omitted due to the change in policy where the AEJ started to publish in 2009. We show the results starting from 2000 in the table, and we did not show estimates from the earlier years for convenience. Table 2 shows the impact of the introduction of the AEJs on academics' representation at lowerranked institutions. The new AEJs created space for authors in low-ranking institutions to publish high-quality but slightly lower-ranked journals. As explained in the data section, we have two different control groups: tier 3 consists of 28 journals, and a new tier 3 consists of 48 journals. Here, we define two different control groups based on the number of citations of articles published in tier 3 journals.

The fixed effect results are also in table 2 . In columns 4 and 5, there is a difference-in-difference model with the journal fixed effect. Statistically, the journal fixed effect captures the time-invariant unobservable factors to the model. In the fixed- 
effect model, the magnitude of the estimated coefficient shrinks: articles average institutional adjusted rank decreases from 2.9 units to 2.6 units for tier 3 with 28 journals. It remains significant for 28 journals of tier 3 as a control group. The articles average institutional adjusted rank also decreases marginally from 2.7 units to 2.6 units and remains significant for 48 journals of tier 3 as a control group in the fixed-effect model. This tells us that there is some evidence that the journals matter for authors. The fixedeffect model also tells us that the journal is a good predictor to capture the individualspecific differences. Both results from the linear difference-in-difference model and the fixed effects confirm positive and statistically significant impacts on the articles average institutional adjusted rank. The fixed effects estimate gives marginally smaller coefficients in our study. The fixed effects estimates of the treatment effect are often smaller than estimates in levels (Angrist \& Kruger, 1999).

Table 2. Impacts of AEJs on articles average institutional ranking of the authors

\begin{tabular}{|c|c|c|c|c|}
\hline \multirow{3}{*}{ Main DID estimates } & \multicolumn{2}{|c|}{ DID } & \multicolumn{2}{|c|}{ DID: Fixed Effect } \\
\hline & $\begin{array}{l}\text { Treatment with } \\
28 \text { journals }\end{array}$ & $\begin{array}{l}\text { Treatment with } \\
48 \text { journals }\end{array}$ & $\begin{array}{l}\text { Treatment with } \\
28 \text { journals }\end{array}$ & $\begin{array}{l}\text { Treatment with } \\
48 \text { journals }\end{array}$ \\
\hline & $\begin{array}{c}2.865 * * * \\
(0.639)\end{array}$ & $\begin{array}{c}2.687 * * * \\
(0.612)\end{array}$ & $\begin{array}{c}2.626 * * * \\
(0.637)\end{array}$ & $\begin{array}{c}2.650 * * * \\
(0.609)\end{array}$ \\
\hline Bootstrap std. error & {$[0.712]$} & {$[0.816]$} & [0.809] & {$[0.800]$} \\
\hline Event study estimates & & & & \\
\hline 2000 & $\begin{array}{c}1.981 \\
(2.013)\end{array}$ & $\begin{array}{c}2.508 \\
(1.920)\end{array}$ & $\begin{array}{c}2.471 \\
(2.000)\end{array}$ & $\begin{array}{c}3.020 \\
(1.906)\end{array}$ \\
\hline 2001 & $\begin{array}{c}7.774 * * * \\
(1.933)\end{array}$ & $\begin{array}{c}7.576^{* * *} \\
(1.841)\end{array}$ & $\begin{array}{c}8.476 * * * \\
(1.920)\end{array}$ & $\begin{array}{c}8.345^{* * *} \\
(1.828)\end{array}$ \\
\hline 2002 & $\begin{array}{l}4.290 * * \\
(1.817)\end{array}$ & $\begin{array}{l}4.111^{* *} \\
(1.732)\end{array}$ & $\begin{array}{c}4.766^{* * * *} \\
(1.806)\end{array}$ & $\begin{array}{c}4.743 * * * \\
(1.720)\end{array}$ \\
\hline 2003 & $\begin{array}{c}6.779 * * * \\
(1.843)\end{array}$ & $\begin{array}{c}6.490 * * * \\
(1.765)\end{array}$ & $\begin{array}{c}7.792 * * * \\
(1.831)\end{array}$ & $\begin{array}{c}7.569 * * * \\
(1.752)\end{array}$ \\
\hline
\end{tabular}




\begin{tabular}{|c|c|c|c|c|}
\hline \multirow[t]{2}{*}{2004} & $4.627 * *$ & $5.207 * * *$ & $4.974 * * *$ & $5.524 * * *$ \\
\hline & $(1.822)$ & (1.739) & (1.809) & $(1.726)$ \\
\hline \multirow[t]{2}{*}{2005} & $7.615 * * *$ & $6.864 * * *$ & $8.471 * * *$ & $7.733 * * *$ \\
\hline & $(1.769)$ & (1.687) & $(1.757)$ & $(1.674)$ \\
\hline \multirow[t]{2}{*}{2006} & $5.475 * * *$ & $6.041 * * *$ & $6.300 * * *$ & $6.946 * * *$ \\
\hline & (1.739) & $(1.662)$ & (1.729) & $(1.650)$ \\
\hline \multirow[t]{2}{*}{2007} & $4.592 * * *$ & $3.856^{* *}$ & $5.482 * * *$ & $4.920 * * *$ \\
\hline & $(1.772)$ & $(1.701)$ & $(1.760)$ & (1.688) \\
\hline 2008 & \multicolumn{4}{|c|}{ (omitted year) } \\
\hline \multirow[t]{2}{*}{2009} & $2.821 *$ & 2.359 & $3.435 * *$ & $3.020 *$ \\
\hline & $(1.700)$ & (1.623) & (1.689) & (1.611) \\
\hline \multirow[t]{2}{*}{2010} & 1.940 & 2.415 & 2.548 & $3.115^{* *}$ \\
\hline & $(1.628)$ & $(1.553)$ & (1.619) & $(1.543)$ \\
\hline \multirow[t]{2}{*}{2011} & $3.926^{* *}$ & $3.957 * *$ & $4.792 * * *$ & $4.863 * * *$ \\
\hline & $(1.652)$ & $(1.577)$ & $(1.643)$ & $(1.567)$ \\
\hline \multirow[t]{2}{*}{2012} & $7.140 * * *$ & $7.178 * * *$ & $7.741 * * *$ & $7.878 * * *$ \\
\hline & $(1.576)$ & $(1.503)$ & $(1.568)$ & $(1.495)$ \\
\hline \multirow[t]{2}{*}{2013} & $9.054 * * *$ & $8.976 * * *$ & $8.601 * * *$ & $8.852 * * *$ \\
\hline & $(1.578)$ & (1.511) & $(1.570)$ & (1.501) \\
\hline \multirow[t]{2}{*}{2014} & $8.077 * * *$ & $7.449 * * *$ & $8.745 * * *$ & $8.359 * * *$ \\
\hline & (1.569) & (1.503) & (1.563) & $(1.495)$ \\
\hline \multirow[t]{2}{*}{2015} & $8.707 * * *$ & $8.600 * * *$ & $9.551 * * *$ & $9.828 * * *$ \\
\hline & (1.639) & (1.574) & (1.635) & $(1.570)$ \\
\hline \multirow[t]{2}{*}{2016} & $3.716^{* *}$ & $3.374 * *$ & $3.931 * *$ & $3.965 * * *$ \\
\hline & $(1.551)$ & (1.484) & $(1.544)$ & $(1.475)$ \\
\hline \multirow[t]{2}{*}{2017} & 2.328 & 1.956 & $2.576^{*}$ & $2.402 *$ \\
\hline & $(1.524)$ & $(1.455)$ & (1.516) & $(1.447)$ \\
\hline \multirow[t]{2}{*}{2018} & $10.835 * * *$ & $10.812 * * *$ & $9.624 * * *$ & $9.873 * * *$ \\
\hline & $(1.680)$ & (1.617) & $(1.670)$ & (1.606) \\
\hline \multirow[t]{2}{*}{2019} & $7.925 * * *$ & $7.314 * * *$ & $5.274 *$ & $4.889 *$ \\
\hline & $(2.759)$ & $(2.680)$ & $(2.746)$ & $(2.665)$ \\
\hline $\mathrm{N}$ & 126,241 & 169,592 & 126,241 & 169,592 \\
\hline
\end{tabular}

Notes: Standard errors in parentheses. $* * * \mathrm{p}<0.01, * * \mathrm{p}<0.05, * \mathrm{p}<0.1$. Estimation process takes into account cluster standard errors at the journal level. Bootstrapping uses Stata's default bootstrapping method using 50 replications. We consider our full data set in this analysis, the year starting from 1991 to 2019.

The analysis uses 295,833 observations, and there are 126,241 observations when we have 28 journals and 169,592 observations when we have 48 journals. Two different main regression model results are presented: difference-in-difference and difference-in-difference with fixed effects. In the fixed effect regression, we consider the journal fixed effect. For convenience, we show results from the year 2000 in this table. 
The standard errors use clustering at the journal level. The standard errors remain stable and shrink marginally in the fixed effect model relative to the linear difference-indifference model. The standard errors decrease from 0.639 to 0.637 and 0.612 to 0.609 in the case of 28 and 48 journals, respectively. The decreased standard error is a trade-off with the fixed-effect model under the linear difference-in-difference model. The fixed effect model's decreasing standard error can be explained as the model uses within journal variation, not between journal variation. Mechanically, the standard error in the fixed effect model is determined by the explanatory power of the model (SSR) and the number of degrees of freedom of the model. ${ }^{26} \mathrm{We}$ also estimate the models using Stata's bootstrapping and mentions the bootstrap standard errors. The bootstrapping is without regard to clustering and is greater than the cluster standard errors at the journal level. Using the bootstrapping in the DID model, the standard errors increase from 0.639 to 0.712 and 0.612 to 0.816 for 28 and 48 journals. In the fixed-effect model, the bootstrapping standard errors increase from 0.637 to 0.809 and 0.609 to 0.800 for 28 and 48 journals, respectively. The standard errors from bootstrapping and conventional methods are close numbers. In this study, using the bootstrapping standard errors does not alter our findings. ${ }^{27}$ Figures 3 and 4 show the treatment effects from the event study design with the difference-in-difference.

\footnotetext{
${ }^{26} \mathrm{SSR}$ refers to the regression sum of squares in the regression model.

${ }^{27}$ Bertrand et al. (2004) argue that many DID papers wrongly calculate the standard errors and thus get misleading results. They suggest using the bootstrapping standard errors when possible.
} 
Figure 3. Difference-in-difference event study impacts of AEJs on authors' from low ranked institutions

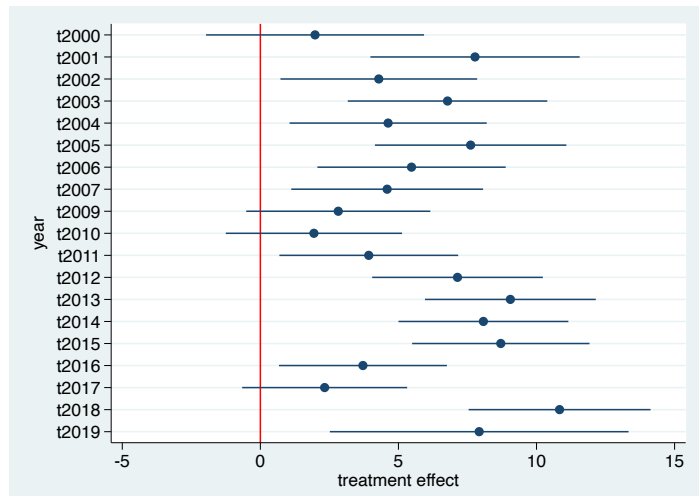

A: treatment group with 28 journals

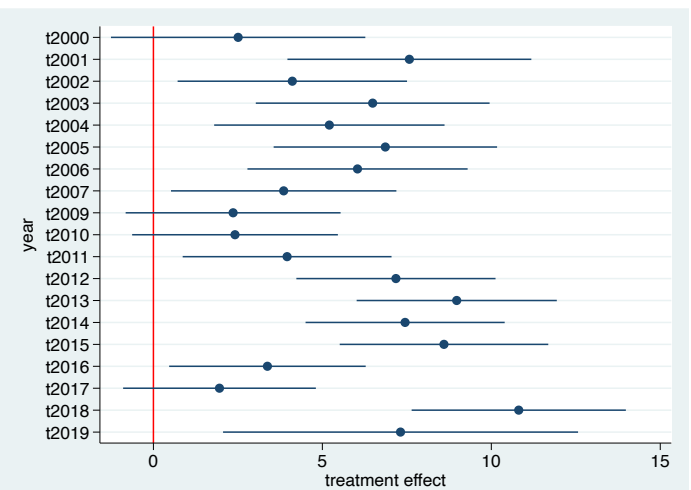

B: treatment group with 48 journals

Notes: Point estimates are the year specific treatment effects that compare prior to 2008 with after 2008 where AEJ starts to publish in 2009, as specified in equation (3). The year 2008 has omitted from the estimates.

Figure 4. Fixed effect difference-in-difference event study impacts of AEJs on authors' from low ranked institutions

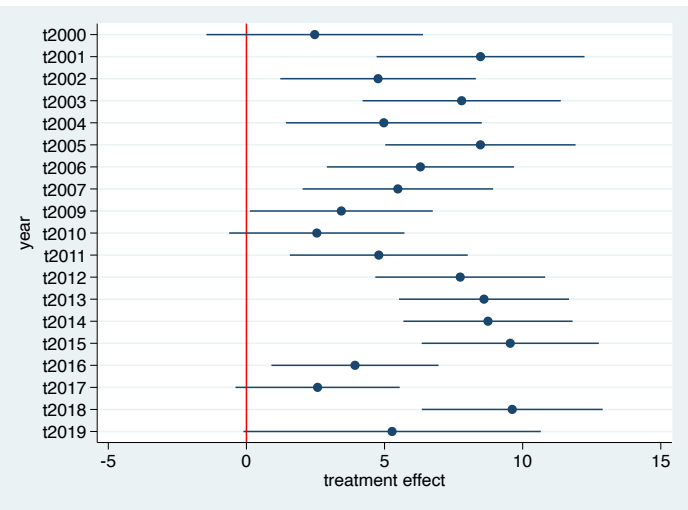

A: treatment group with 28 journals

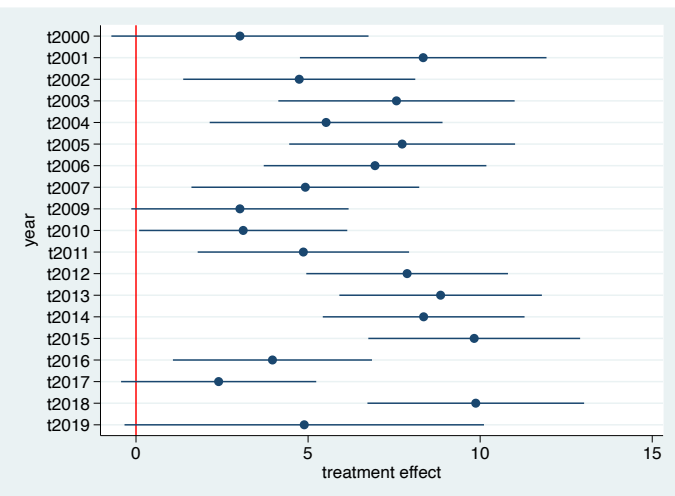

B: treatment group with 48 journals

Notes: Point estimates are the year specific treatment effects that compare prior to 2008 with after 2008 where AEJ starts to publish in 2009, as specified in equation (3). The year 2008 has omitted from the estimates. 


\section{Robustness of Main Treatment Effects}

We have three robustness tests of our main treatment effects. We defined the outcome variable of the articles average institutional adjusted ranking of authors in three different ways to check the sensitivity. In the first approach, we consider minimum author rank. Minimum author rank is defined so that if one article has coauthors, the minimum author rank is defined by the author's lowest rank among all authors. For example, if a paper has three coauthors of $\mathrm{X}, \mathrm{Y}$, and $\mathrm{Z}$, we look at their adjusted ranks and take the minimum rank. Minimum rank reflects authors from high-ranked institutions. This approach could be a good predictor of the model as we ask the authors who have the minimum rank and how their rankings are affected by the publication of a brand new journal. In other words, we are looking at the authors who were gained by the AEJs and who got to publish the new journal. Table 3 tests our main results' robustness that tells us that the AEJs publication helps articles average adjusted institutional ranking of authors to increase their rank by 4.9 points to 5.7 points in difference-in-difference regressions. These are significant at $p<0.01$.

Table 3. Impacts of AEJs on articles average institutional ranking of the authors (minimum author' rank)

\begin{tabular}{|c|c|c|c|c|}
\hline & \multicolumn{2}{|c|}{ DID } & \multicolumn{2}{|c|}{ DID: Fixed Effect } \\
\hline & $\begin{array}{l}\text { Treatment with } \\
28 \text { journals }\end{array}$ & $\begin{array}{c}\text { Treatment with } \\
48 \text { journals }\end{array}$ & $\begin{array}{l}\text { Treatment with } \\
28 \text { journals }\end{array}$ & $\begin{array}{l}\text { Treatment with } \\
48 \text { journals }\end{array}$ \\
\hline Main DID estimates & $\begin{array}{c}5.484 * * * \\
(0.702)\end{array}$ & $\begin{array}{c}5.651 * * * \\
(0.680)\end{array}$ & $\begin{array}{c}4.879 * * * \\
(0.697)\end{array}$ & $\begin{array}{c}5.201 * * * \\
(0.675)\end{array}$ \\
\hline Bootstrap std. error & {$[0.611]$} & {$[0.701]$} & {$[0.674]$} & {$[0.800]$} \\
\hline Event study estimates & & & & \\
\hline 2000 & $\begin{array}{l}4.940 * * \\
(2.206)\end{array}$ & $\begin{array}{l}5.344 * * \\
(2.130)\end{array}$ & $\begin{array}{l}5.244 * * \\
(2.184)\end{array}$ & $\begin{array}{c}5.542 * * * \\
(2.107)\end{array}$ \\
\hline 2001 & $14.296^{* * *}$ & $13.863 * * *$ & $14.680 * * *$ & $14.222 * * *$ \\
\hline
\end{tabular}




\begin{tabular}{|c|c|c|c|c|}
\hline & (2.117) & (2.043) & (2.096) & $(2.020)$ \\
\hline \multirow[t]{2}{*}{2002} & $8.046^{* * *}$ & $7.544 * * *$ & $8.336^{* * *}$ & $7.989 * * *$ \\
\hline & (1.991) & $(1.921)$ & $(1.972)$ & (1.901) \\
\hline \multirow[t]{2}{*}{2003} & $11.664 * * *$ & $11.252^{* * *}$ & $12.552 * * *$ & $12.169^{* * *}$ \\
\hline & $(2.019)$ & (1.958) & (1.999) & (1.936) \\
\hline \multirow[t]{2}{*}{2004} & $10.001 * * *$ & $10.360 * * *$ & $10.478 * * *$ & $10.695^{* * *}$ \\
\hline & (1.996) & $(1.929)$ & $(1.975)$ & $(1.907)$ \\
\hline \multirow[t]{2}{*}{2005} & $15.605^{* * *}$ & $14.021 * * *$ & $16.135^{* * *}$ & $14.445^{* * *}$ \\
\hline & (1.938) & $(1.871)$ & (1.919) & $(1.850)$ \\
\hline \multirow[t]{2}{*}{2006} & $11.044 * * *$ & $11.413^{* * *}$ & $11.493 * * *$ & $11.856^{* * *}$ \\
\hline & $(1.906)$ & (1.844) & $(1.887)$ & $(1.824)$ \\
\hline \multirow[t]{2}{*}{2007} & $5.568 * * *$ & $5.383 * * *$ & $6.175^{* * *}$ & $6.122 * * *$ \\
\hline & $(1.941)$ & (1.888) & $(1.921)$ & $(1.866)$ \\
\hline 2008 & \multicolumn{4}{|c|}{ (omitted year) } \\
\hline \multirow[t]{2}{*}{2009} & $4.918^{* * *}$ & $4.124 * *$ & $5.201 * * *$ & $4.387 * *$ \\
\hline & $(1.863)$ & $(1.800)$ & $(1.844)$ & $(1.780)$ \\
\hline \multirow[t]{2}{*}{2010} & $6.801^{* * *}$ & $7.813^{* * *}$ & $6.992 * * *$ & $7.945^{* * *}$ \\
\hline & $(1.784)$ & $(1.723)$ & $(1.767)$ & $(1.705)$ \\
\hline \multirow[t]{2}{*}{2011} & $8.904 * * *$ & $9.099 * * *$ & $9.304 * * *$ & $9.379 * * *$ \\
\hline & $(1.809)$ & (1.749) & (1.794) & $(1.732)$ \\
\hline \multirow[t]{2}{*}{2012} & $14.393 * * *$ & $14.908^{* * *}$ & $14.543 * * *$ & $14.958^{* * *}$ \\
\hline & $(1.727)$ & $(1.668)$ & $(1.713)$ & $(1.652)$ \\
\hline \multirow[t]{2}{*}{2013} & $15.832 * * *$ & $16.458^{* * *}$ & $14.916^{* * *}$ & $15.770^{* * *}$ \\
\hline & $(1.729)$ & (1.676) & $(1.714)$ & $(1.659)$ \\
\hline \multirow[t]{2}{*}{2014} & $16.949 * * *$ & $16.139 * * *$ & $16.938^{* * *}$ & $16.296^{* * *}$ \\
\hline & (1.719) & $(1.668)$ & $(1.706)$ & $(1.653)$ \\
\hline \multirow[t]{2}{*}{2015} & $15.061 * * *$ & $15.692 * * *$ & $15.331^{* * *}$ & $16.287 * * *$ \\
\hline & $(1.795)$ & $(1.747)$ & $(1.785)$ & $(1.735)$ \\
\hline \multirow[t]{2}{*}{2016} & $9.936 * * *$ & $9.314 * * *$ & $9.457 * * *$ & $9.140 * * *$ \\
\hline & (1.699) & $(1.646)$ & $(1.685)$ & (1.631) \\
\hline \multirow[t]{2}{*}{2017} & $4.669 * * *$ & $4.642 * * *$ & $4.347^{* * *}$ & $4.429 * * *$ \\
\hline & $(1.670)$ & (1.614) & $(1.656)$ & (1.599) \\
\hline \multirow[t]{2}{*}{2018} & $16.434 * * *$ & $16.751^{* * *}$ & $15.453 * * *$ & $15.991 * * *$ \\
\hline & $(1.841)$ & (1.794) & $(1.823)$ & $(1.775)$ \\
\hline \multirow[t]{2}{*}{2019} & $16.894 * * *$ & $16.318^{* * *}$ & $14.048 * * *$ & $13.527 * * *$ \\
\hline & $(3.023)$ & $(2.973)$ & (2.998) & $(2.945)$ \\
\hline $\mathrm{N}$ & 126,241 & 169,592 & 126,241 & 169,592 \\
\hline
\end{tabular}

Notes: Standard errors in parentheses. ${ }^{* * *} \mathrm{p}<0.01,{ }^{* *} \mathrm{p}<0.05,{ }^{*} \mathrm{p}<0.1$. Estimation process takes into account cluster standard errors at the journal level. Bootstrapping uses Stata's default bootstrapping method using 50 replications. We consider our full data set in this analysis, the year starting from 1991. The analysis uses 295,833 observations, and there are 126,241 observations when we have 28 journals and 169,592 
observations when we have 48 journals. Two different main regression model results are presented: difference-in-difference and difference-in-difference with fixed effects. In the fixed effect regression, we consider journal fixed effect. For convenience, we show results from the year 2000 in this table.

In table 3, we have linear difference-in-difference and the difference-in-difference with fixed effect regressions. The coefficients are 5.5 with the control group of tier 3 consists of 28 journals and 5.7 with the control group of tier 3 consists of 48 journals, respectively. These are significant at $p<0.01$. The difference-in-difference coefficients in the first model show that the treatment effects are higher than our main model in table 2. Since we define the outcome variable only with the minimum adjusted rank, we see an increase in articles average adjusted institutional ranking of authors by the AEJs publication. The effects are twofold greater than the main model in table 2. We expect this increment since we define the outcome variable with the minimum authors' rank. The assumption is that if we assign most of our authors with their minimum rank, then they are opportunistic. The conventional standard error decreases from 0.702 to 0.680 when considering the control group with 28 journals and 48 journals in the DID model. The coefficients in difference-in-difference with the fixed effect regression decrease marginally when we use the journal fixed effect. The articles average adjusted institutional ranking of authors increases by 4.9 points with the 28 journals and 5.2 with 48 journals in the fixed effect. These are marginally lower than our first regression of linear difference-indifference. The bootstrapped standard error decreases marginally when we use the fixed effect relative to the conventional standard errors. 
Figure 5. Difference-in-difference event study impacts of AEJs on authors' from low ranked institutions considering minimum author rank

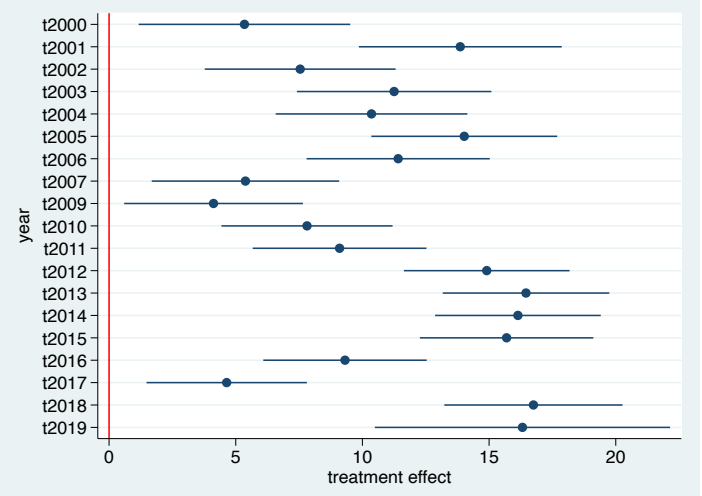

A: treatment group with 28 journals

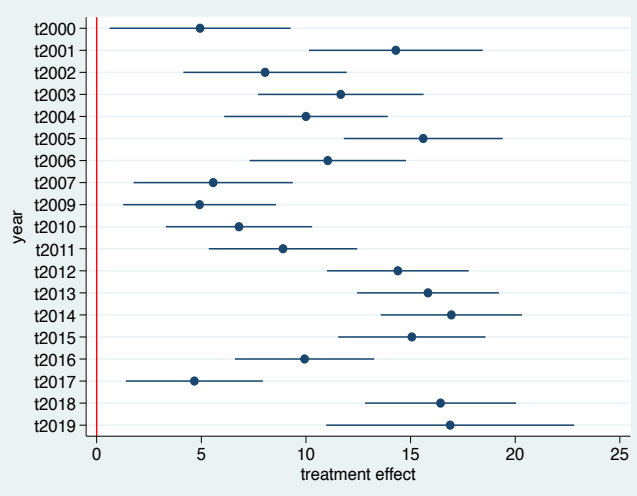

B: treatment group with 48 journals

Notes: Point estimates are the year specific treatment effects that compare prior to 2008 with after 2008 where AEJ starts to publish in 2009, as specified in equation (3). The year 2008 has omitted from the estimates.

The event study point estimates from 2009 are consistently high enough to have a causal effect in various years. These estimates are statistically significant at $p<0.01$. The pre-AEJ estimates are presented in figure 5 and support the parallel trends assumption between the treatment and control group. The event study point estimates for fixed effects regression in the last two columns are also high in various years. These are also significant at $p<0.01$. These estimates are presented in Figure 6 and support the parallel trends assumption between treatment and control groups. 
Figure 6. Fixed effect difference-in-difference event study impacts of AEJs on authors from low ranked institutions considering minimum author rank

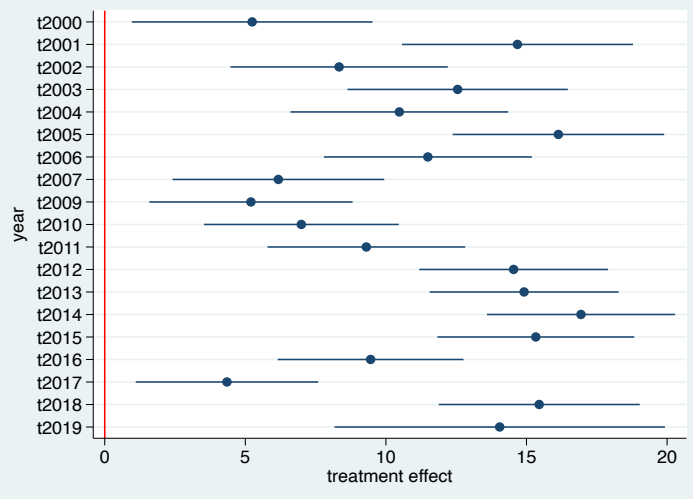

A: treatment group with 28 journals

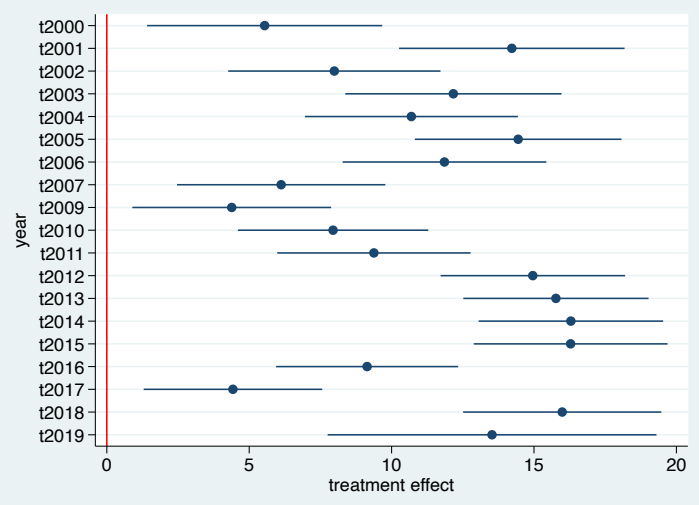

B: treatment group with 48 journals

Notes: Point estimates are the year specific treatment effects that compare prior to 2008 with after 2008 where AEJ starts to publish in 2009, as specified in equation (3). The year 2008 has omitted from the estimates. In the fixed effect regression, we consider journal fixed effect.

In our robustness check, we move to the second procedure to define the outcome variable by the maximum rank. To define the maximum rank, we consider the articles average adjusted institutional ranking of authors with the highest rank from a co-authored article. For example, if three authors $\mathrm{X}, \mathrm{Y}$, and $\mathrm{Z}$, write a paper, we consider the articles rank from the maximum rank from these three. The idea here is to see if we take the maximum ranks of how the AEJs influence the authors' publication opportunities. A high article rank tells us that the author is far from the prestigious institutions in our sample data set. This second robustness check is valuable since we want to see how the authors' publication opportunities from low-ranked institutions changed by the AEJs. We may expect the opposite result by using the maximum article rank relative to the minimum article rank. 
Table 4. Impacts of AEJs on articles average institutional ranking of the authors (maximum author' rank)

\begin{tabular}{|c|c|c|c|c|}
\hline & \multicolumn{2}{|c|}{ DID } & \multicolumn{2}{|c|}{ DID: Fixed Effect } \\
\hline & $\begin{array}{l}\text { Treatment with } \\
28 \text { journals }\end{array}$ & $\begin{array}{l}\text { Treatment with } \\
48 \text { journals }\end{array}$ & $\begin{array}{l}\text { Treatment with } \\
28 \text { journals }\end{array}$ & $\begin{array}{c}\text { Treatment with } \\
48 \text { journals }\end{array}$ \\
\hline Main DID estimates & $\begin{array}{c}0.682 \\
(0.440)\end{array}$ & $\begin{array}{c}0.277 \\
(0.417)\end{array}$ & $\begin{array}{l}0.770^{*} \\
(0.438)\end{array}$ & $\begin{array}{c}0.497 \\
(0.414)\end{array}$ \\
\hline Bootstrap std. error & {$[0.577]$} & {$[0.592]$} & [ 0.582$]$ & {$[0.510]$} \\
\hline Event study estimates & & & & \\
\hline 2000 & $\begin{array}{l}-2.017 \\
(1.388)\end{array}$ & $\begin{array}{l}-1.266 \\
(1.310)\end{array}$ & $\begin{array}{l}-1.489 \\
(1.377)\end{array}$ & $\begin{array}{l}-0.655 \\
(1.298)\end{array}$ \\
\hline 2001 & $\begin{array}{l}2.944 * * \\
(1.332)\end{array}$ & $\begin{array}{l}2.914 * * \\
(1.256)\end{array}$ & $\begin{array}{c}3.719 * * * \\
(1.322)\end{array}$ & $\begin{array}{c}3.788 * * * \\
(1.245)\end{array}$ \\
\hline 2002 & $\begin{array}{c}2.011 \\
(1.252)\end{array}$ & $\begin{array}{c}1.933 \\
(1.181)\end{array}$ & $\begin{array}{l}2.511 * * \\
(1.244)\end{array}$ & $\begin{array}{l}2.538 * * \\
(1.171)\end{array}$ \\
\hline 2003 & $\begin{array}{l}3.194^{* *} \\
(1.270)\end{array}$ & $\begin{array}{l}2.902^{* *} \\
(1.204)\end{array}$ & $\begin{array}{c}4.098 * * * \\
(1.261)\end{array}$ & $\begin{array}{c}3.875^{* * *} \\
(1.193)\end{array}$ \\
\hline 2004 & $\begin{array}{c}0.435 \\
(1.256)\end{array}$ & $\begin{array}{c}1.104 \\
(1.186)\end{array}$ & $\begin{array}{c}0.622 \\
(1.246)\end{array}$ & $\begin{array}{c}1.314 \\
(1.175)\end{array}$ \\
\hline 2005 & $\begin{array}{l}-0.780 \\
(1.219)\end{array}$ & $\begin{array}{l}-0.709 \\
(1.150)\end{array}$ & $\begin{array}{c}0.090 \\
(1.210)\end{array}$ & $\begin{array}{c}0.219 \\
(1.140)\end{array}$ \\
\hline 2006 & $\begin{array}{c}-2.830^{* *} \\
(1.199)\end{array}$ & $\begin{array}{c}-2.371 * * \\
(1.134)\end{array}$ & $\begin{array}{l}-1.928 \\
(1.190)\end{array}$ & $\begin{array}{l}-1.383 \\
(1.124)\end{array}$ \\
\hline 2007 & $\begin{array}{c}1.540 \\
(1.221)\end{array}$ & $\begin{array}{c}0.839 \\
(1.161)\end{array}$ & $\begin{array}{l}2.428^{* *} \\
(1.212)\end{array}$ & $\begin{array}{c}1.859 \\
(1.150)\end{array}$ \\
\hline 2008 & & & year) & \\
\hline 2009 & $\begin{array}{l}-0.352 \\
(1.172)\end{array}$ & $\begin{array}{l}-0.700 \\
(1.107)\end{array}$ & $\begin{array}{c}0.440 \\
(1.163)\end{array}$ & $\begin{array}{c}0.078 \\
(1.097)\end{array}$ \\
\hline 2010 & $\begin{array}{l}-0.260 \\
(1.122)\end{array}$ & $\begin{array}{l}-0.227 \\
(1.059)\end{array}$ & $\begin{array}{c}0.543 \\
(1.115)\end{array}$ & $\begin{array}{c}0.666 \\
(1.051)\end{array}$ \\
\hline 2011 & $\begin{array}{l}-0.386 \\
(1.138)\end{array}$ & $\begin{array}{l}-0.406 \\
(1.075)\end{array}$ & $\begin{array}{c}0.646 \\
(1.131)\end{array}$ & $\begin{array}{c}0.694 \\
(1.067)\end{array}$ \\
\hline 2012 & $\begin{array}{c}1.669 \\
(1.086)\end{array}$ & $\begin{array}{c}1.422 \\
(1.025)\end{array}$ & $\begin{array}{c}2.490 * * \\
(1.080)\end{array}$ & $\begin{array}{l}2.381 * * \\
(1.018)\end{array}$ \\
\hline 2013 & $\begin{array}{c}3.102 * * * \\
(1.088)\end{array}$ & $\begin{array}{l}2.495^{* *} \\
(1.031)\end{array}$ & $\begin{array}{c}3.068 * * * \\
(1.081)\end{array}$ & $\begin{array}{c}2.719 * * * \\
(1.022)\end{array}$ \\
\hline 2014 & $\begin{array}{c}0.110 \\
(1.082)\end{array}$ & $\begin{array}{l}-0.563 \\
(1.025)\end{array}$ & $\begin{array}{c}1.144 \\
(1.076)\end{array}$ & $\begin{array}{c}0.637 \\
(1.018)\end{array}$ \\
\hline 2015 & 0.826 & 0.636 & $1.973 *$ & $2.070^{*}$ \\
\hline
\end{tabular}




\begin{tabular}{lcccc} 
& $(1.129)$ & $(1.074)$ & $(1.126)$ & $(1.069)$ \\
2016 & 0.266 & -0.132 & 0.885 & 0.772 \\
& $(1.069)$ & $(1.012)$ & $(1.063)$ & $(1.005)$ \\
2017 & -0.740 & -1.099 & -0.146 & -0.351 \\
& $(1.051)$ & $(0.993)$ & $(1.044)$ & $(0.985)$ \\
2018 & $2.894^{* *}$ & $2.624^{* *}$ & 1.798 & 1.702 \\
& $(1.158)$ & $(1.103)$ & $(1.150)$ & $(1.094)$ \\
2019 & 0.562 & 0.068 & -1.530 & -1.837 \\
& $(1.902)$ & $(1.828)$ & $(1.891)$ & $(1.815)$ \\
$\mathrm{N}$ & 126,241 & 169,592 & 126,241 & 169,592 \\
\hline
\end{tabular}

Notes: Standard errors in parentheses. ${ }^{* * *} \mathrm{p}<0.01,{ }^{*} \mathrm{p}<0.05, * \mathrm{p}<0.1$. Estimation process takes into account cluster standard errors at the journal level. Bootstrapping uses Stata's default bootstrapping method using 50 replications. We consider our full data set in this analysis, the year starting from 1991. The analysis uses 295,833 observations, and there are 126,241 observations when we have 28 journals and 169,592 observations when we have 48 journals. Two different main regression model results are presented: difference-in-difference and difference-in-difference with fixed effects. In the fixed effect regression, we consider journal fixed effect. For convenience, we show results from the year 2000 in this table.

The point estimates in table 4 tell us that the articles that change the rank from low-ranked institutions affiliated authors actually failed to increase the rank due to the AEJ publication. This is essentially telling us that the authors' from low-ranked institutions failed to publish high-ranked journals. The rank increases marginally and is not statistically significant, except the FE with 28 journals at $p<0.1$.

Figure 7. Difference-in-difference event study impacts of AEJs on authors' from low ranked institutions considering maximum author rank

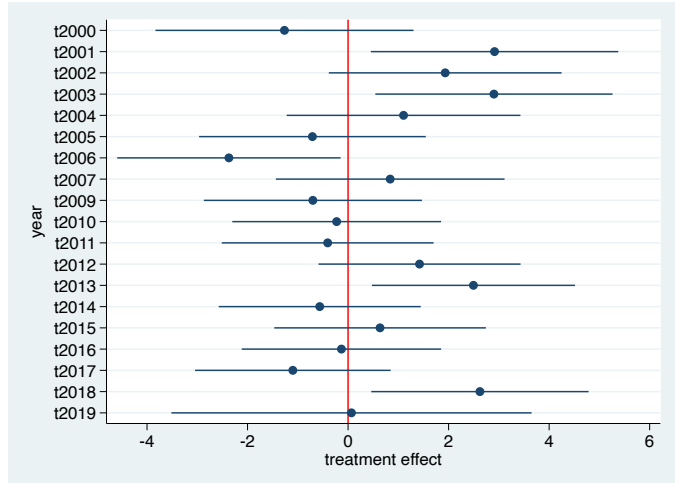

A: treatment group with 28 journals

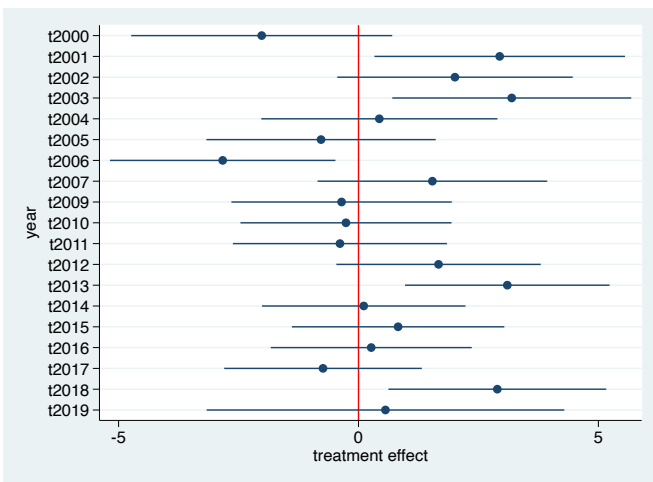

B: treatment group with 48 journals 
Notes: Point estimates are the year specific treatment effects that compare prior to 2008 with after 2008 where AEJ starts to publish in 2009, as specified in equation (3). The year 2008 has omitted from the estimates.

Figure 8. Fixed effect difference-in-difference event study impacts of AEJs on authors' from low ranked institutions considering maximum author rank

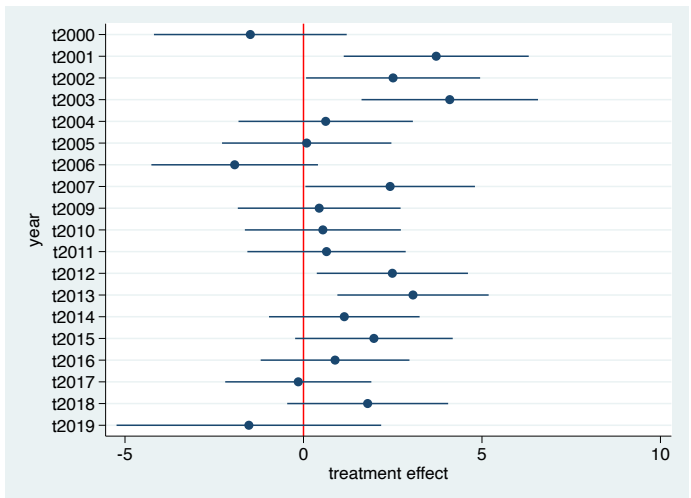

A: treatment group with 28 journals

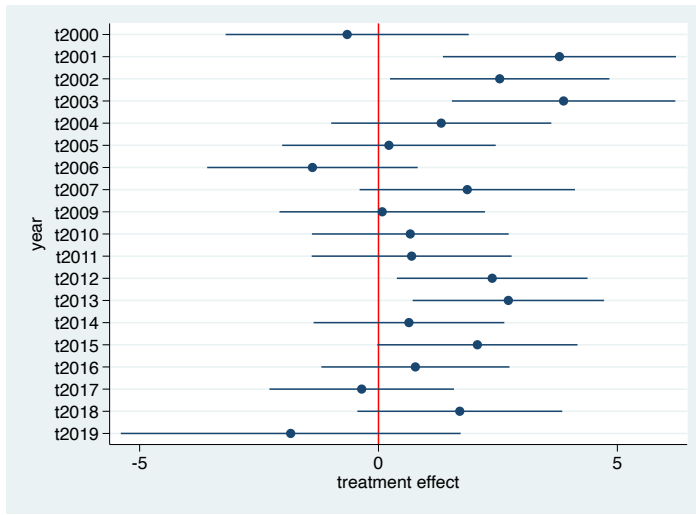

B: treatment group with 48 journals

Notes: Point estimates are the year specific treatment effects that compare prior to 2008 with after 2008 where AEJ starts to publish in 2009, as specified in equation (3). The year 2008 has omitted from the estimates. In the fixed effect regression, we consider journal fixed effect.

In the third robustness check, we define the outcome variable as the "percent of articles published by someone who had never published there in the tier 3 journals before." Percent of articles by a single author is defined as the number of papers published by person ' $\mathrm{X}$ ' divided by the total number of articles in our sample window. In doing this process, we consider those authors who have published their papers more frequently in 28 journals (in the first measure by citation count) or 48 (in the second measure by citation count) as explained in step 6 in the data section. In the analysis, we have tier 1 journals as control and tier 3 journals as treatment. The new measure of the percent of the article published will allow us to see how authors' overall performance varies due to the AEJs. 
Table 5. Impacts of AEJs on articles average institutional ranking of the authors (percent of articles published)

\begin{tabular}{|c|c|c|c|c|}
\hline & \multicolumn{2}{|c|}{ DID } & \multicolumn{2}{|c|}{ DID: Fixed Effect } \\
\hline & $\begin{array}{l}\text { Treatment with } \\
28 \text { journals }\end{array}$ & $\begin{array}{l}\text { Treatment with } \\
48 \text { journals }\end{array}$ & $\begin{array}{l}\text { Treatment with } \\
28 \text { journals }\end{array}$ & $\begin{array}{l}\text { Treatment with } \\
48 \text { journals }\end{array}$ \\
\hline Main DID estimates & $\begin{array}{c}0.0004 * * * \\
(0.0000)\end{array}$ & $\begin{array}{c}0.0006^{* * *} \\
(0.0000)\end{array}$ & $\begin{array}{c}0.0005 * * * \\
(0.0000)\end{array}$ & $\begin{array}{c}0.0006^{* * *} \\
(0.0000)\end{array}$ \\
\hline Bootstrap std. error & {$[0.0001]$} & {$[0.0000]$} & [0.0001] & {$[0.0001]$} \\
\hline Event study estimates & & & & \\
\hline 2000 & $\begin{array}{l}-0.0003 \\
(0.0003)\end{array}$ & $\begin{array}{l}-0.0003 \\
(0.0003)\end{array}$ & $\begin{array}{l}-0.0003 \\
(0.0003)\end{array}$ & $\begin{array}{l}-0.0004 \\
(0.0003)\end{array}$ \\
\hline 2001 & $\begin{array}{c}-0.0007 * * \\
(0.0003)\end{array}$ & $\begin{array}{c}-0.0006^{* *} \\
(0.0003)\end{array}$ & $\begin{array}{c}-0.0007 * * \\
(0.0003)\end{array}$ & $\begin{array}{c}-0.0006^{* *} \\
(0.0003)\end{array}$ \\
\hline 2002 & $\begin{array}{l}-0.0002 \\
(0.0003)\end{array}$ & $\begin{array}{l}-0.0001 \\
(0.0003)\end{array}$ & $\begin{array}{l}-0.0002 \\
(0.0003)\end{array}$ & $\begin{array}{l}-0.0002 \\
(0.0003)\end{array}$ \\
\hline 2003 & $\begin{array}{c}0.0001 \\
(0.0003)\end{array}$ & $\begin{array}{c}0.0001 \\
(0.0003)\end{array}$ & $\begin{array}{l}-0.0000 \\
(0.0003)\end{array}$ & $\begin{array}{l}-0.0001 \\
(0.0003)\end{array}$ \\
\hline 2004 & $\begin{array}{l}-0.0004 \\
(0.0003)\end{array}$ & $\begin{array}{l}-0.0003 \\
(0.0003)\end{array}$ & $\begin{array}{l}-0.0004 \\
(0.0003)\end{array}$ & $\begin{array}{l}-0.0004 \\
(0.0003)\end{array}$ \\
\hline 2005 & $\begin{array}{c}0.0002 \\
(0.0003)\end{array}$ & $\begin{array}{c}0.0002 \\
(0.0003)\end{array}$ & $\begin{array}{l}-0.0000 \\
(0.0003)\end{array}$ & $\begin{array}{l}-0.0000 \\
(0.0002)\end{array}$ \\
\hline 2006 & $\begin{array}{l}-0.0003 \\
(0.0003)\end{array}$ & $\begin{array}{l}-0.0002 \\
(0.0002)\end{array}$ & $\begin{array}{l}-0.0004 * \\
(0.0003)\end{array}$ & $\begin{array}{l}-0.0004 * \\
(0.0002)\end{array}$ \\
\hline 2007 & $\begin{array}{l}-0.0001 \\
(0.0003)\end{array}$ & $\begin{array}{c}0.0000 \\
(0.0003)\end{array}$ & $\begin{array}{l}-0.0003 \\
(0.0003)\end{array}$ & $\begin{array}{l}-0.0002 \\
(0.0003)\end{array}$ \\
\hline 2008 & & (om & ear) & \\
\hline 2009 & $\begin{array}{l}-0.0001 \\
(0.0003)\end{array}$ & $\begin{array}{c}0.0001 \\
(0.0002)\end{array}$ & $\begin{array}{l}-0.0001 \\
(0.0003)\end{array}$ & $\begin{array}{l}-0.0000 \\
(0.0002)\end{array}$ \\
\hline 2010 & $\begin{array}{l}0.0005^{*} \\
(0.0002)\end{array}$ & $\begin{array}{c}0.0006^{* *} \\
(0.0002)\end{array}$ & $\begin{array}{c}0.0004 \\
(0.0002)\end{array}$ & $\begin{array}{l}0.0004^{*} \\
(0.0002)\end{array}$ \\
\hline 2011 & $\begin{array}{c}0.0006 * * \\
(0.0003)\end{array}$ & $\begin{array}{c}0.0007^{* * *} \\
(0.0002)\end{array}$ & $\begin{array}{l}0.0005 * * \\
(0.0002)\end{array}$ & $\begin{array}{l}0.0005 * * \\
(0.0002)\end{array}$ \\
\hline 2012 & $\begin{array}{c}0.0001 \\
(0.0002)\end{array}$ & $\begin{array}{c}0.0003 \\
(0.0002)\end{array}$ & $\begin{array}{l}-0.0000 \\
(0.0002)\end{array}$ & $\begin{array}{c}0.0001 \\
(0.0002)\end{array}$ \\
\hline 2013 & $\begin{array}{c}0.0001 \\
(0.0002)\end{array}$ & $\begin{array}{c}0.0004 \\
(0.0002)\end{array}$ & $\begin{array}{c}0.0002 \\
(0.0002)\end{array}$ & $\begin{array}{l}0.0004 * \\
(0.0002)\end{array}$ \\
\hline 2014 & $\begin{array}{l}0.0004 * \\
(0.0002)\end{array}$ & $\begin{array}{c}0.0007 * * * \\
(0.0002)\end{array}$ & $\begin{array}{c}0.0004 \\
(0.0002)\end{array}$ & $\begin{array}{l}0.0005^{* *} \\
(0.0002)\end{array}$ \\
\hline 2015 & 0.0002 & $0.0005^{* *}$ & 0.0003 & 0.0003 \\
\hline
\end{tabular}




$\begin{array}{lcccc} & (0.0002) & (0.0002) & (0.0002) & (0.0002) \\ 2016 & 0.0007^{* * *} & 0.0009^{* * *} & 0.0006^{* * *} & 0.0008^{* * *} \\ & (0.0002) & (0.0002) & (0.0002) & (0.0002) \\ 2017 & 0.0008^{* * *} & 0.0011^{* * *} & 0.0008^{* * *} & 0.0010^{* * *} \\ & (0.0002) & (0.0002) & (0.0002) & (0.0002) \\ 2018 & 0.0007^{* * *} & 0.0010^{* * *} & 0.0009^{* * *} & 0.0011^{* * *} \\ & (0.0003) & (0.0002) & (0.0003) & (0.0002) \\ & 0.0002 & 0.0005 & 0.0005 & 0.0006 \\ 2019 & (0.0004) & (0.0004) & (0.0004) & (0.0004) \\ 126,241 & 169,592 & 126,241 & 169,592 \\ \mathrm{~N} & \text { Notes: Standard errors in parentheses. *** p<0.01, ** p<0.05, * p<0.1. Estimation process takes into } \\ \text { account cluster standard errors at the journal level. Bootstrapping uses Stata's default bootstrapping method } \\ \text { using 50 replications. We consider our full data set in this analysis, the year starting from 1991. The } \\ \text { analysis uses 295,833 observations, and there are 126,241 observations when we have 28 journals and } \\ \begin{array}{l}169,592 \text { observations when we have 48 journals. Two different main regression model results are } \\ \text { presented: difference-in-difference and difference-in-difference with fixed effects. In the fixed effect }\end{array} \\ \text { regression, we consider journal fixed effect. For convenience, we show results from the year 2000 in this } \\ \text { table. }\end{array}$

In the difference-in-difference estimates, we see that articles average adjusted institutional ranking of authors from low-ranked institutions increased their publications range of $0.04 \%$ to $0.06 \%$. These are, in fact, high numbers in terms of the number of articles published due to AEJs. In 28 journals, $0.04 \%$ is equivalent to 50 articles, and for 48 journals, this is 68 articles. The estimates are significant at $p<0.01$. The event study estimates show that the years after the AEJs are positive, and authors from low-ranked institutions get the opportunity to publish their papers. The conventional and bootstrapped standard errors are low numbers due to the outcome variable's use in the percent term. 
Figure 9. Difference-in-difference event study impacts of AEJs on authors from low ranked institutions considering percent of articles published
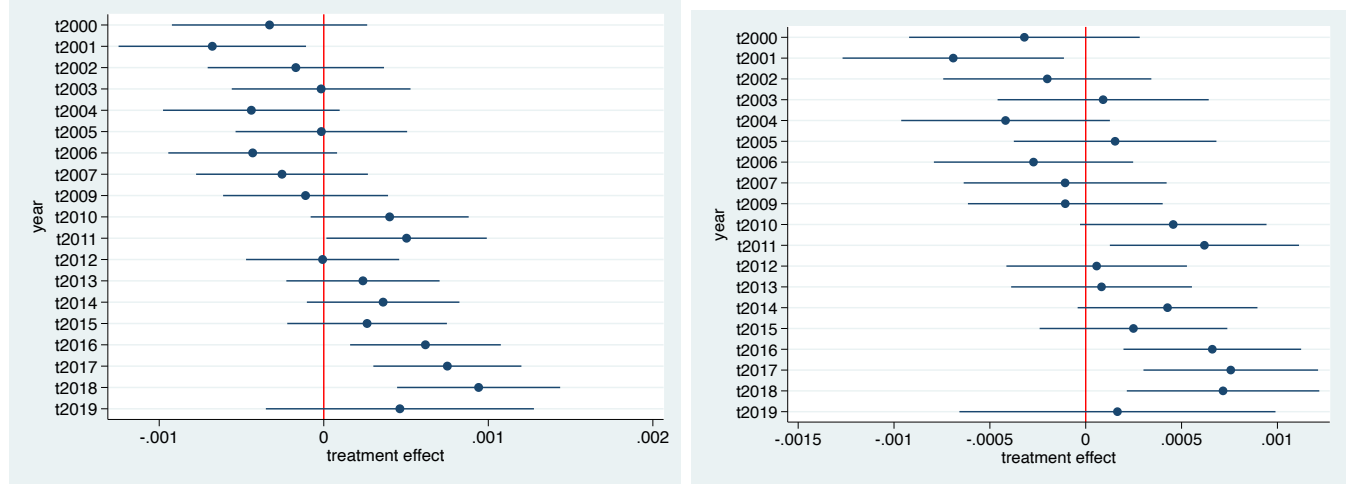

A: treatment group with 28 journals B: treatment group with 48 journals

Notes: Point estimates are the year specific treatment effects that compare prior to 2008 with after 2008 where AEJ starts to publish in 2009, as specified in equation (3). The year 2008 has omitted from the estimates.

Figure 10. Fixed effect difference-in-difference event study impacts of AEJs on authors from low ranked institutions considering percent of articles published

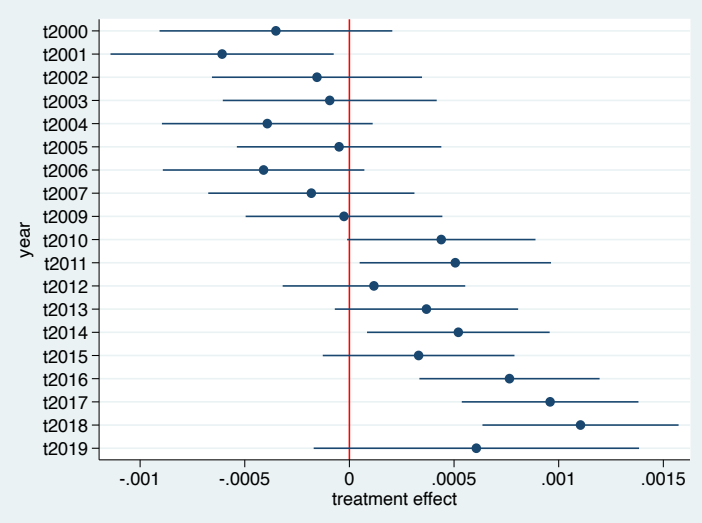

A: treatment group with 28 journals

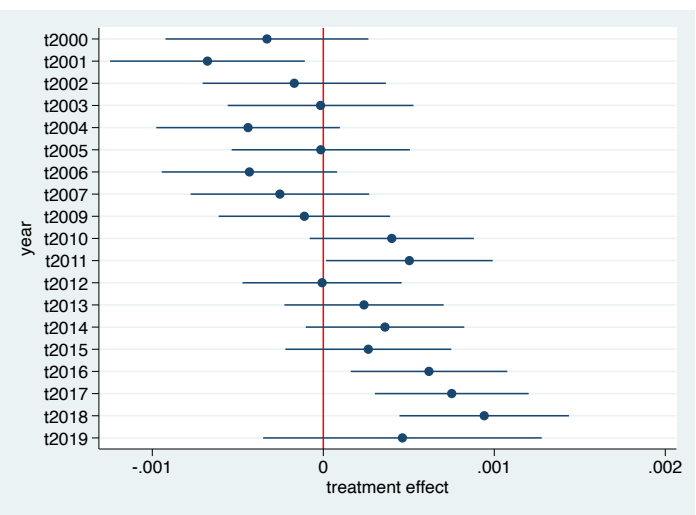

B: treatment group with 48 journals

Notes: Point estimates are the year specific treatment effects that compare prior to 2008 with after 2008 where AEJ starts to publish in 2009, as specified in equation (3). The year 2008 has omitted from the estimates. In the fixed effect regression, we consider journal fixed effect. 


\section{Conclusion}

This study examines the effect of a new journal series in economics on publications by academicians from low-ranked institutions. Authors from the low-ranked institutions are our point of interest since we rely on whether the authors from low-ranked institutions can publish at higher-ranked journals due to the new AEJ series. The difference-in-difference estimates show weak evidence of the effect of a new journal (AEJs) in economics on authors from low-rank institutions. In academia, people earlier believed that economics is a saturated field in getting a position, and a new journal can help them overcome the problem. Since publishing in economics journal is always hard than most other academic disciplines. Journal from the apex professional organization in economics may help professionals from low-rank institutions. However, this is not the case as people expect to rationalize the discipline and its future.

We find that there are weak causal effects of publishing a new journal for the authors from low-ranked institutions in economics. Several issues can rationalize the findings. The publication opportunities are limited to the high demand from academicians and researchers in economics. Still, there is a limited number of journals considered prestigious publication avenues by most institutions. Sometimes people refer to the top 100 journals as the right places to publish research outcomes and consider promotion. Moreover, there is an increasing lag period to publish an article in economics relative to other disciplines. Academicians at the early stage of their careers suffer the most due to this delay in submission and publication. Considering the delay, authors may consider publishing their research in journals where it takes a short period. However, the relatively quicker period journals are low ranked. The present study considers the first 120 journals 
and tries to look at the effect of AEJs. Based on the analysis, we can infer that there is no strong effect of a new journal on these 120 journals.

The current study provides insights into the role of new journals in economics, and policymakers can take advantage of the findings. In economics, the discipline needs more journals that can compete with high-ranked journals and make room for academicians and researchers. 


\section{References}

Akerlof, G. A. (2020). Sins of Omission and the Practice of Economics. Journal of Economic Literature, 58(2), 405-418.

Anauati, M. V., Galiani, S., \& Gálvez, R. H. (2020). Differences in citation patterns across journal tiers: The case of economics. Economic Inquiry, 58(3), 1217-1232.

Angrist, J. D., \& Kruger, A. (1999). Empirical strategies in labor economics, Handbook of Labor Economics, Volume 3, Edited by O. Ashenfelter and D. Card.

Bertrand, M., Duflo, E., \& Mullainathan, S. (2004). How much should we trust differences-in-differences estimates? The Quarterly Journal of Economics, 119(1), 249-275.

Björk, B.-C., \& Solomon, D. (2013). The publishing delay in scholarly peer-reviewed journals. Journal of Informetrics, 7(4), 914-923.

Card, D., \& DellaVigna, S. (2013). Nine facts about top journals in economics. Journal of Economic Literature, 51(1), 144-161.

Colussi, T. (2018). Social ties in academia: A friend is a treasure. Review of Economics and Statistics, 100(1), 45-50.

Cunningham, S. (2018). Causal Inference: The Mixtape. Unpublished Manuscript.

Einav, L., \& Yariv, L. (2006). What's in a surname? The effects of surname initials on academic success. Journal of Economic Perspectives, 20(1), 175-187.

Ellison, G. (2002). The slowdown of the economics publishing process. Journal of Political Economy, 110(5), 947-993.

Ellison, G. (2011). Is peer review in decline? Economic Inquiry, 49(3), 635-657. 
Frey, B. S. (2003). Publishing as prostitution?-Choosing between one's own ideas and academic success. Public Choice, 116(1-2), 205-223.

Gibson, J. (2014, June 6). No top fives, no worries? VoxEU.Org. https://voxeu.org/article/no-top-fives-no-worries

Gibson, J., Anderson, D. L., \& Tressler, J. (2014). Which journal rankings best explain academic salaries? Evidence from the University of California. Economic Inquiry, 52(4), 1322-1340.

Goodman-Bacon, A. (2018). Difference-in-differences with variation in treatment timing (No. 0898-2937). National Bureau of Economic Research.

Goyal, S., Van Der Leij, M. J., \& Moraga-González, J. L. (2006). Economics: An emerging small world. Journal of Political Economy, 114(2), 403-412.

Gurantz, O. (2020). What does free community college buy? Early impacts from the Oregon Promise. Journal of Policy Analysis and Management, 39(1), 11-35.

Heckman, J. J., \& Moktan, S. (2020). Publishing and promotion in economics: The tyranny of the top five. Journal of Economic Literature, 58(2), 419-470.

Ma, C., Li, Y., Guo, F., \& Si, K. (2019). The citation trap: Papers published at year-end receive systematically fewer citations. Journal of Economic Behavior \& Organization, 166, 667-687.

Osterloh, M., \& Frey, B. S. (2020). How to avoid borrowed plumes in academia. Research Policy, 49(1), 103831.

Sandler, D. H., \& Sandler, R. (2014). Multiple event studies in public finance and labor economics: A simulation study with applications. Journal of Economic and Social Measurement, 39(1-2), 31-57. 
Simple Impact Factors for Economics Journals | IDEAS/RePEc. (n.d.). Retrieved May 14, 2019, from https://ideas.repec.org/top/top.journals.simple.html

Trivedi, P. K. (1993). An analysis of publication lags in econometrics. Journal of Applied Econometrics, 93-100. 


\section{Appendix}

Figure A1. Journal articles after reshaped into one row per author-article (\% annually)

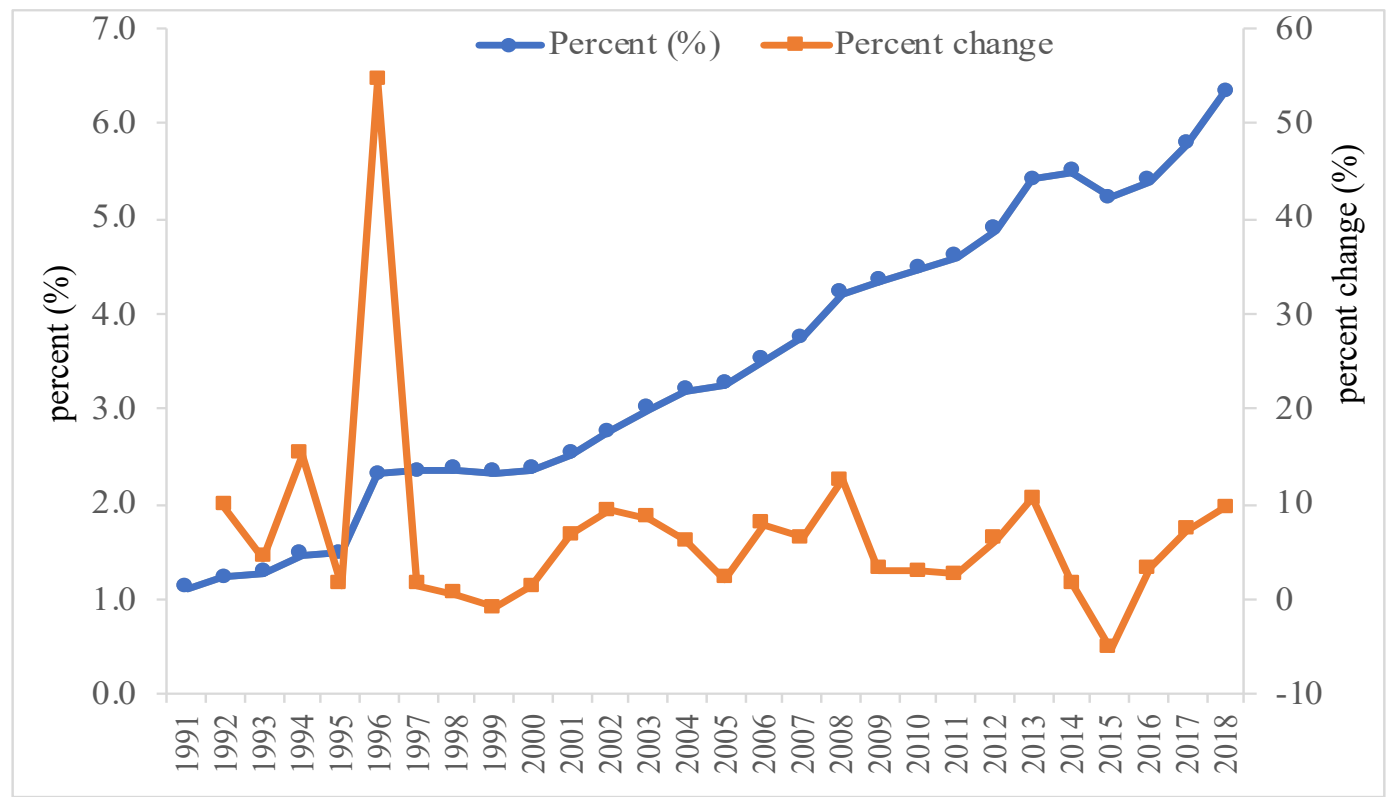

Source: Authors' calculation based on the dataset.

Notes: The figure uses observations after reshaping into one row per author-article. We dropped the year 2019 due to a lack of full year's observation. 2019 could be a misleading year to show as the observations drop sharply in this year. The line with circles marker represents the percent of articles in a year relative to total articles over the sample period. The line with a square marker represents the percent change in a year relative to the previous year.

Figure A2. Highest similarity score $(>0.5)$ per institution

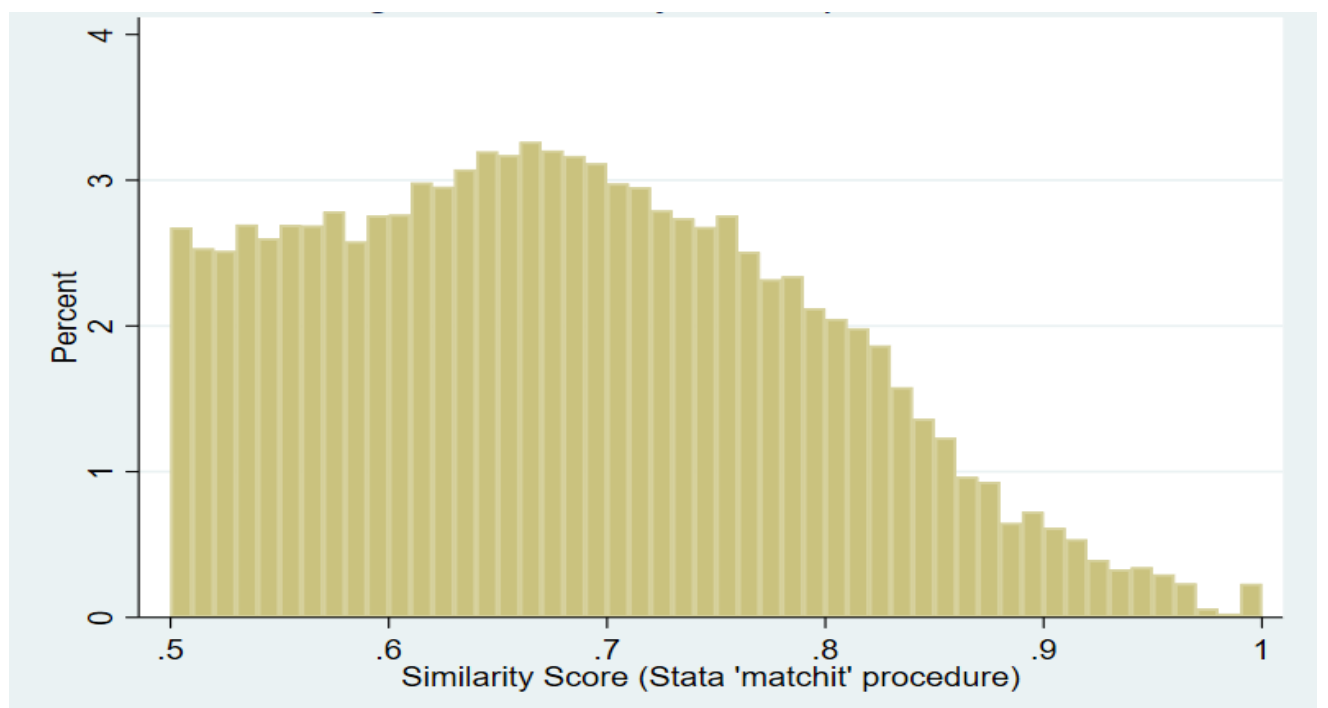

Notes: The histogram is a matching result for the highest similscore for each journal affiliation. We get data from a match of institutional rank taken from IDEAS/RePEc linked to Scopus data using Stata's 'matchit' procedure. We consider a similarity score greater than 0.5 for a reasonable standard of matching procedure. 
Figure A3. Ability of Stata's 'matchit' procedure to link author's stated affiliation to institutional rank

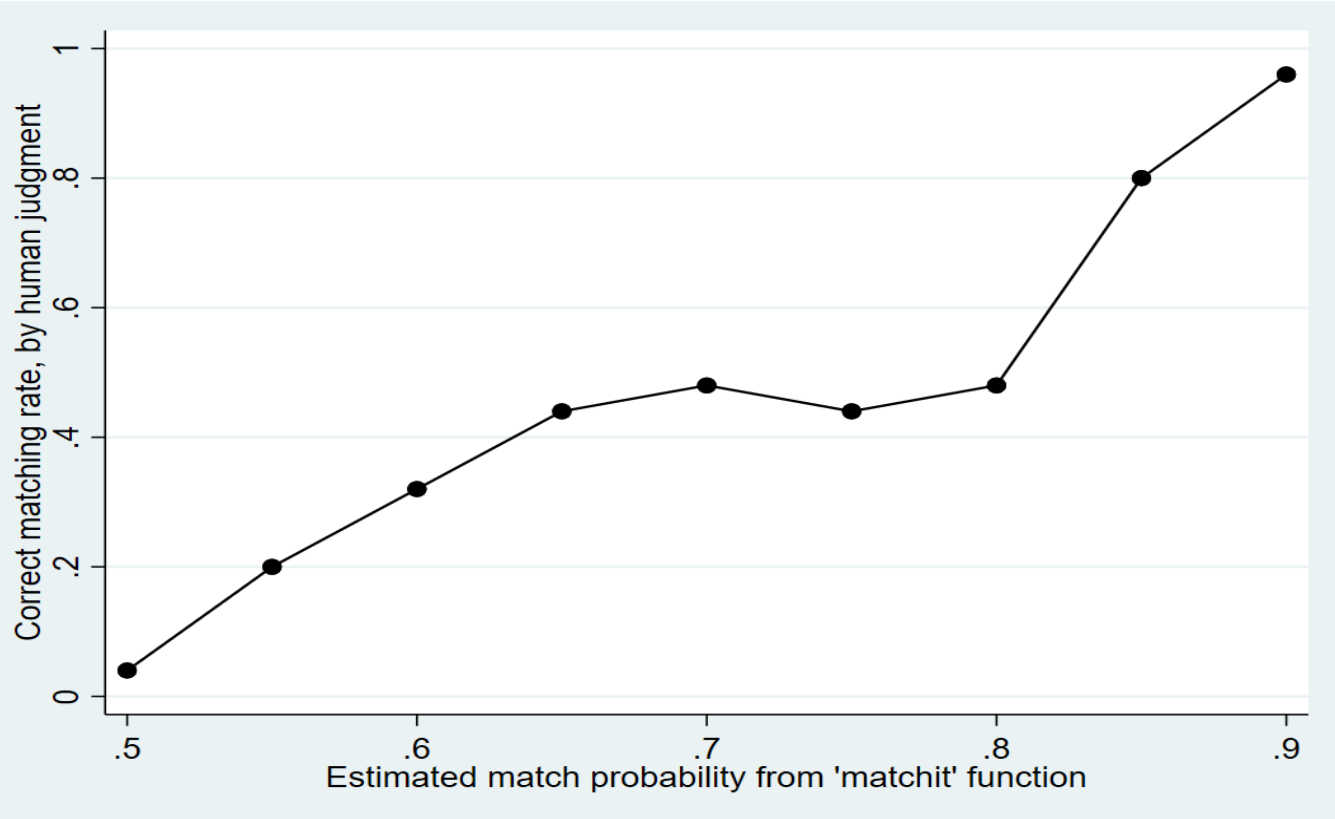

Notes: Data derives from a match of institutional rank taken from IDEAS/RePEc linked to Scopus data using Stata's ' matchit' procedure. For each bin of 0.05 points (i.e., $0.50-0.549 ; 0.55-0.599$ ) a random sample of 25 observations was selected, and human judgment was used to verify the accuracy.

Figure A4. Average institutional rank of authors per journal article

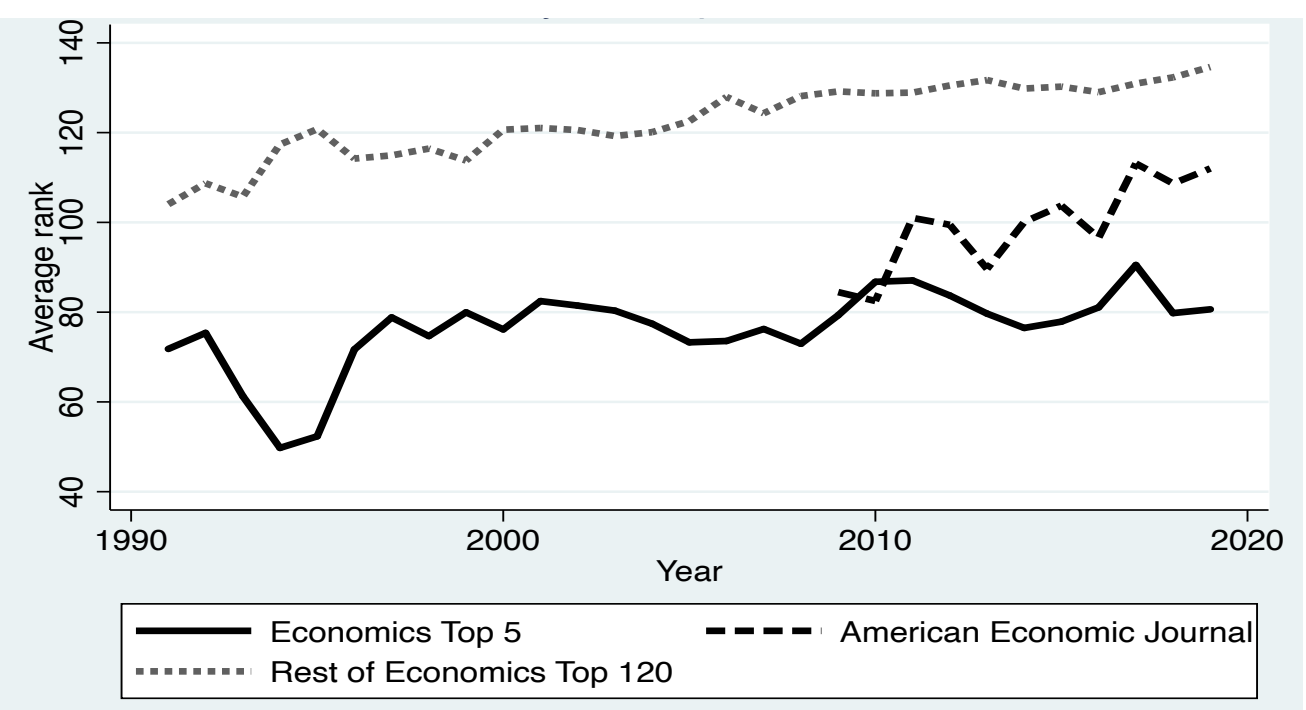

Notes: Data include all publications from the top 120 Economics journals, as measured by IDEAS/RePEc data and downloaded from Scopus in June 2019. Institutional rank was taken from IDEAS/RePEc and linked to Scopus data using Stata's 'matchit' procedure. Calculations are the average institutional rank per published journal article. "Top 5" refers to American Economic Review, Quarterly Journal of Economics, Econometrica, Review of Economic Studies, and the Journal of Political Economy. The American Economic Journal category includes all four journals in the top 120 journals: Applied Economics, Economic Policy, Macroeconomics, and Microeconomics. 
Table A1: Journals by groups

\begin{tabular}{|c|c|c|c|c|c|}
\hline \multirow[b]{2}{*}{ Sl. } & \multirow[b]{2}{*}{ Journals } & \multirow{2}{*}{$\begin{array}{c}\text { Tier } 1 \\
\text { (Top 5) } \\
\text { Journals }\end{array}$} & \multirow{2}{*}{$\begin{array}{c}\text { Tier } 2 \\
\text { (AEJs) } \\
\text { Journals }\end{array}$} & \multicolumn{2}{|c|}{ Tier 3} \\
\hline & & & & $\begin{array}{c}28 \\
\text { Journals } \\
\end{array}$ & $\begin{array}{c}48 \\
\text { Journals } \\
\end{array}$ \\
\hline 1 & Quarterly Journal of Economics & 1 & 0 & 0 & 0 \\
\hline 2 & Journal of Economic Literature & 0 & 0 & 0 & 1 \\
\hline 3 & Journal of Political Economy & 1 & 0 & 0 & 0 \\
\hline 4 & Econometrica & 1 & 0 & 0 & 0 \\
\hline 5 & Journal of Economic Growth & 0 & 0 & 0 & 1 \\
\hline 6 & Journal of Financial Economics & 0 & 0 & 1 & 1 \\
\hline 7 & Review of Economic Studies & 1 & 0 & 0 & 0 \\
\hline 8 & Journal of Finance & 0 & 0 & 1 & 1 \\
\hline 9 & Journal of Economic Perspectives & 0 & 0 & 1 & 1 \\
\hline 10 & American Economic Review & 1 & 0 & 0 & 0 \\
\hline 11 & Economic Policy & 0 & 0 & 0 & 1 \\
\hline 12 & Review of Financial Studies & 0 & 0 & 0 & 1 \\
\hline 13 & Journal of Monetary Economics & 0 & 0 & 1 & 1 \\
\hline 14 & Journal of Labor Economics & 0 & 0 & 1 & 1 \\
\hline 15 & Brookings Papers on Economic Activity & 0 & 0 & 0 & 1 \\
\hline 16 & Journal of Econometrics & 0 & 0 & 1 & 1 \\
\hline 17 & Economic Journal & 0 & 0 & 1 & 1 \\
\hline 18 & American Economic Journal: Macroeconomics & 0 & 1 & 0 & 0 \\
\hline 19 & RAND Journal of Economics & 0 & 0 & 1 & 1 \\
\hline 20 & Journal of the European Economic Association & 0 & 0 & 1 & 1 \\
\hline 21 & Review of Economics and Statistics & 0 & 0 & 1 & 1 \\
\hline 23 & Journal of Business & 0 & 0 & 0 & 0 \\
\hline 24 & Journal of Applied Econometrics & 0 & 0 & 0 & 1 \\
\hline 25 & $\begin{array}{l}\text { American Economic Journal: Applied } \\
\text { Economics }\end{array}$ & 0 & 1 & 0 & 0 \\
\hline 26 & Journal of International Economics & 0 & 0 & 1 & 1 \\
\hline 27 & Journal of Business and Economic Statistics & 0 & 0 & 1 & 1 \\
\hline 28 & Journal of Human Resources & 0 & 0 & 1 & 1 \\
\hline 30 & World Bank Economic Review & 0 & 0 & 0 & 1 \\
\hline 31 & Journal of Public Economics & 0 & 0 & 1 & 1 \\
\hline 32 & Journal of Financial Intermediation & 0 & 0 & 0 & 0 \\
\hline 33 & Econometrics Journal & 0 & 0 & 0 & 0 \\
\hline 34 & Journal of Accounting and Economics & 0 & 0 & 0 & 0 \\
\hline 35 & Experimental Economics & 0 & 0 & 0 & 0 \\
\hline 36 & Journal of Development Economics & 0 & 0 & 1 & 1 \\
\hline 37 & $\begin{array}{l}\text { Journal of Environmental Economics and } \\
\text { Management }\end{array}$ & 0 & 0 & 0 & 1 \\
\hline
\end{tabular}




\begin{tabular}{|c|c|c|c|c|c|}
\hline 38 & Journal of Economic Surveys & 0 & 0 & 0 & 0 \\
\hline 39 & Review of Economic Dynamics & 0 & 0 & 1 & 1 \\
\hline 40 & Journal of Law and Economics & 0 & 0 & 0 & 1 \\
\hline 41 & Journal of Economic Theory & 0 & 0 & 1 & 1 \\
\hline 42 & Annual Review of Economics & 0 & 0 & 0 & 0 \\
\hline 44 & European Economic Review & 0 & 0 & 1 & 1 \\
\hline 45 & International Economic Review & 0 & 0 & 1 & 1 \\
\hline 46 & World Bank Research Observer & 0 & 0 & 0 & 0 \\
\hline 47 & Journal of Risk and Uncertainty & 0 & 0 & 1 & 1 \\
\hline 48 & Journal of Money, Credit and Banking & 0 & 0 & 1 & 1 \\
\hline 49 & International Journal of Central Banking & 0 & 0 & 0 & 0 \\
\hline 50 & IMF Economic Review & 0 & 0 & 0 & 0 \\
\hline 51 & Oxford Bulletin of Economics and Statistics & 0 & 0 & 0 & 0 \\
\hline 52 & American Economic Journal: Economic Policy & 0 & 1 & 0 & 0 \\
\hline 53 & Journal of Law, Economics, and Organization & 0 & 0 & 0 & 1 \\
\hline 54 & Journal of International Business Studies & 0 & 0 & 0 & 0 \\
\hline 55 & Journal of Urban Economics & 0 & 0 & 1 & 1 \\
\hline 56 & Journal of International Money and Finance & 0 & 0 & 0 & 0 \\
\hline 57 & Mathematical Finance & 0 & 0 & 0 & 0 \\
\hline 58 & Journal of Health Economics & 0 & 0 & 1 & 1 \\
\hline 59 & Journal of Business Venturing & 0 & 0 & 0 & 0 \\
\hline 60 & Journal of Industrial Economics & 0 & 0 & 0 & 1 \\
\hline 61 & Journal of Financial Markets & 0 & 0 & 0 & 0 \\
\hline 62 & Journal of Financial and Quantitative Analysis & 0 & 0 & 0 & 0 \\
\hline 63 & Research Policy & 0 & 0 & 0 & 0 \\
\hline 64 & Labour Economics & 0 & 0 & 0 & 1 \\
\hline 66 & Journal of Population Economics & 0 & 0 & 0 & 0 \\
\hline 67 & Journal of Financial Econometrics & 0 & 0 & 0 & 0 \\
\hline 68 & Latin American Journal of Economics & 0 & 0 & 0 & 0 \\
\hline 69 & Journal of Economic Dynamics and Control & 0 & 0 & 1 & 1 \\
\hline 71 & Journal of Empirical Finance & 0 & 0 & 0 & 0 \\
\hline 72 & Journal of Banking and Finance & 0 & 0 & 0 & 0 \\
\hline 73 & Oxford Review of Economic Policy & 0 & 0 & 0 & 1 \\
\hline 75 & Journal of Economic Geography & 0 & 0 & 0 & 0 \\
\hline 77 & International Journal of Industrial Organization & 0 & 0 & 0 & 1 \\
\hline 78 & Management Science & 0 & 0 & 0 & 0 \\
\hline 79 & Scandinavian Journal of Economics & 0 & 0 & 0 & 1 \\
\hline 80 & Oxford Economic Papers & 0 & 0 & 0 & 0 \\
\hline 81 & Journal of Economics and Management Strategy & 0 & 0 & 0 & 1 \\
\hline
\end{tabular}




\begin{tabular}{|c|c|c|c|c|c|}
\hline 82 & Games and Economic Behavior & 0 & 0 & 1 & 1 \\
\hline 83 & Economics and Politics & 0 & 0 & 0 & 0 \\
\hline 84 & Industrial and Corporate Change & 0 & 0 & 0 & 0 \\
\hline 85 & Econometric Reviews & 0 & 0 & 0 & 0 \\
\hline 86 & Stata Journal & 0 & 0 & 0 & 0 \\
\hline 87 & OECD Journal: Economic Studies & 0 & 0 & 0 & 0 \\
\hline 88 & Econometric Theory & 0 & 0 & 1 & 1 \\
\hline 89 & Economica & 0 & 0 & 0 & 1 \\
\hline 90 & International Finance & 0 & 0 & 0 & 0 \\
\hline 91 & Marketing Science & 0 & 0 & 0 & 0 \\
\hline 92 & Journal of Accounting Research & 0 & 0 & 0 & 0 \\
\hline 93 & Review of Environmental Economics and Policy & 0 & 0 & 0 & 0 \\
\hline 94 & Energy Economics & 0 & 0 & 0 & 0 \\
\hline 95 & American Journal of Agricultural Economics & 0 & 0 & 0 & 1 \\
\hline 96 & Financial Management & 0 & 0 & 0 & 0 \\
\hline 97 & Finance and Stochastics & 0 & 0 & 0 & 0 \\
\hline 98 & International Tax and Public Finance & 0 & 0 & 0 & 0 \\
\hline 99 & Journal of Economic Behavior and Organization & 0 & 0 & 1 & 1 \\
\hline 100 & Resource and Energy Economics & 0 & 0 & 0 & 0 \\
\hline 101 & Quantitative Economics & 0 & 0 & 0 & 0 \\
\hline 102 & European Journal of Political Economy & 0 & 0 & 0 & 0 \\
\hline 103 & Review of Finance & 0 & 0 & 0 & 0 \\
\hline 104 & Land Economics & 0 & 0 & 0 & 0 \\
\hline 105 & B.E. Journal of Macroeconomics & 0 & 0 & 0 & 0 \\
\hline 106 & Journal of Consumer Research & 0 & 0 & 0 & 0 \\
\hline 107 & Journal of Financial Services Research & 0 & 0 & 0 & 0 \\
\hline 108 & Annual Review of Financial Economics & 0 & 0 & 0 & 0 \\
\hline 109 & Small Business Economics & 0 & 0 & 0 & 0 \\
\hline 110 & Journal of Productivity Analysis & 0 & 0 & 0 & 0 \\
\hline 111 & Journal of Economic Inequality & 0 & 0 & 0 & 0 \\
\hline 112 & Annals of Economics and Finance & 0 & 0 & 0 & 0 \\
\hline 113 & Energy Journal & 0 & 0 & 0 & 0 \\
\hline 114 & Environmental and Resource Economics & 0 & 0 & 0 & 0 \\
\hline 115 & Regional Science and Urban Economics & 0 & 0 & 0 & 1 \\
\hline 116 & Economic Inquiry & 0 & 0 & 1 & 1 \\
\hline 117 & American Economic Journal: Microeconomics & 0 & 1 & 0 & 0 \\
\hline 118 & International Journal of Finance and Economics & 0 & 0 & 0 & 0 \\
\hline 119 & Canadian Journal of Economics & 0 & 0 & 0 & 1 \\
\hline 120 & Journal of the Royal Statistical Society & 0 & 0 & 0 & 0 \\
\hline
\end{tabular}


Notes: We refer top 5 journals as tier 1 , and these are the authors in the control group for the analysis where they publish. The "Top 5" include American Economic Review, Econometrica, Journal of Political Economy, Quarterly Journal of Economics, and Review of Economic Studies. The AEJ is a group of four reviewed academic journals with fields of Applied Economics, Economic Policy, Macroeconomics, and Microeconomics. These are tier 2 journals. Tier 3 is the list of journals, and we consider authors who publish at tier 3 and are in the treatment group. We have two treatment groups: one that includes 28 journals "tier 3(28)," and the other one consists of 48 journals "tier 3(48)." In these four groups, "1" refers to the journals that fall under that group. " 0 " refers that the journal does not fall under that group. 
Tapas Paul, Ph.D., is a senior lecturer at the Department of Economics, Independent University, Bangladesh. His research interests include labor economics, development economics, poverty and inequality, and policy analysis. Tapas holds a bachelor's and master's degree in Economics from Jahangirnagar University, Bangladesh. Tapas holds a second master's degree in Economics from the University of Illinois at Urbana Champaign and a doctorate in public policy from the University of Missouri, Columbia. Prior to his doctoral studies, Tapas worked for research and academic positions in Bangladesh. 